\author{
U.S. DEPARTMENT OF THE INTERIOR \\ U.S. GEOLOGICAL SURVEY
}

\title{
AUDIO-MAGNETOTELLURIC GEOELECTRIC SOUNDINGS IN JACKSON HOLE, WYOMING
}

\section{By}

Robert M. Senterfit ${ }^{1}$, David L. Campbel $1^{1}$, B. Thomas Nolan', and Marilla senterfit ${ }^{2}$

\section{Open-File Report 95-239}

This report is preliminary and has not been reviewed for conformity with U.S. Geological Survey editorial standards or with the North American Stratigraphic Code. Any use of trade, firm or product names is for descriptive purposes only and does not imply endorsement by the U.S. Government.

Denver, Colorado

1995

\footnotetext{
${ }^{1}$ USGS Branch of Geophysics, MS 964, Denver Federal Center, Denver CO 80225-0046

${ }^{2}$ USGS Water Resources Division, 2617 E. Lincolnway, Suite B, Cheyenne WY 82001
} 


\section{AUDIO-MAGNETOTELLURIC GEOELECTRIC SOUNDINGS IN JACKSON HOLE, WYOMING}

by Robert M. Senterfit, David L. Campbell, B. Thomas Nolan, and Marilla Senterfit

\section{INTRODUCTION}

In late May and early June of 1994, the USGS made 81 audiomagnetotelluric (AMT) soundings in Jackson Hole, a valley in Teton County, northwestern Wyoming. In September, 1994, we repeated measurements at 6 of the earlier AMT sites and added one new AMT site. Most of the sounding sites were located in fields on hay farms and cattle ranches in the southern part of the valley. The AMT work was done to help detect geoelectrical features that might reflect geologic and hydrologic conditions in Jackson Hole, and was part of a wider series of studies there that included time-domain geoelectric soundings and hydrologic studies (Campbell and Nolan, 1995).

The basic principles of the AMT method correspond to those of the magnetotelluric (MT) method (Cagniard, 1953; Vozoff, 1972; Vozoff and others, 1963). AMT signals, however, occur at higher frequencies and originate mainly from atmospheric disturbances (spherics) rather than the lower frequencies used in MT (typically in the range of 0.001 to $1,000 \mathrm{~Hz}$ ) that originate from ionospheric phenomena. General references on AMT method include Strangway and others (1973), Vozoff (1991), and Zonge and Hughes (1991). Reports describing AMT work to investigate geologic structures and lithologic relations include that by Hoover and others (1976); Hermance and others (1984); Leary and Phinney (1974); Stanley (1982); Long (1985); and Fitterman and others (1988).

The AMT electromagnetic induction method is typically used to delineate geoelectrical features in the upper $0.5-2.0 \mathrm{~km}$ of the earth's crust. Soundings were made using distant field sources, mostly natural sources in the frequency range from 4.5 $\mathrm{Hz}$ to $27,000 \mathrm{~Hz}$. The data for each station consist of scalar measurements of discrete frequencies for two orthogonal magnetic and electric field pairs. The two pairs of data are denoted $\mathrm{N}-\mathrm{S}$ and E-W, reflecting the (true geographic) North-South and EastWest orientation of electrode pairs that measured the electric fields. The particular AMT equipment and data reduction system used for this study is described by Hoover and others (1976, 1978) and Hoover and Long (1976).

Figure 1 shows the locations of the 82 AMT soundings. All of the locations are on Jackson Lake, WY and Jackson, WY maps, scale 1:1,100,000. Locations outside the Teton National Park are on the Teton Village, WY, and Jackson, WY, maps, scale 1:24,000. The sounding curves for $\mathrm{N}-\mathrm{S}$ and $\mathrm{E}-\mathrm{W}$ components at each station along with their corresponding data sets are shown in Appendix $A$. Interpreted electric structures at each station, found using a one-dimensional method (Bostick, 1977) are shown in Appendix B. 
ACKNOWLEDGEMENT: We thank the Teton County Natural Resource District, the Jackson Hole Baseline Research Foundation, and the USGS Water Resources Division cooperative program for funding this work.

REFERENCES

Bostick, F.X., Jr., 1977, A simple and almost exact method of MT analysis: Proceedings of the University of Utah Workshop on Electrical Methods in Geothermal Exploration, p. 175-188.

Cagniard, L., 1953, Basic theory of the magnetotelluric method: Geophysics, v. 18, no. 3, p. 605-635.

Campbell, D.L., and Nolan, B.T., in prep., Time-domain geoelectric soundings in Jackson Hole, Wyoming: Part A results and Part B - data: U.S. Geological Survey Open-file Report.

Fitterman, D.V., Stanley, W.D., and Bisdorf, R.J., 1988, Electric structure of Newberry volcano, Oregon: Journal of Geophysical Research, v. 93, no. B9, p. 10119-10134.

Hermance, J.F., Slocum, W.M., and Neuman, G.A., 1984, The Long Valley/Mono Basin volcanic complex--A preliminary magnetotelluric and magnetic variation interpretation: Journal of Geophysical Research, v. 89, p. 8325-8337.

Hoover, D.B., Frischknecht, F.C., and Tippens, C.L., 1976, Audiomagnetotelluric soundings as a reconnaissance exploration technique in Long Valley, California: Journal of Geophysical Research, v. 81, p. 801-809.

Hoover, D.B., and Long, C.L., 1976, Audio-magnetotelluric methods in reconnaissance geothermal exploration: Proceedings, 2nd U.N. Symposium, Developmental Geothermal Resources, p. 10591064.

Hoover, D.B., Long, C.L., and Senterfit, R.M., 1978, Some results from audio-magnetotelluric investigations in geothermal areas: Geophysics, v. 43, no. 7, p. 1501-1514.

Leary, P., and Phinney, R.A., 1974, A magnetotelluric traverse across the Yellowstone region: Geophysical Research Letters, v. 1 , no. 6, p. 265-268.

Long, C.L., 1985, Regional audio-magnetotelluric study of the Questa caldera, New Mexico: Journal of Geophysical Research, v. 90, p. 11270-11274.

Stanley, W.D.. 1982, Magnetotelluric soundings on the Idaho 
National Engineering Lab Facility, Idaho: Journal of Geophysical Research, v. 87, no. B4, p. 2686-2691.

Strangway, D.W., Swift, C.M. Jr., and Holmer, R.C., 1973, The application of audio-frequency magnetotellurics (AMT) to mineral exploration: Geophysics, v.38, p. 1159-1175.

Vozoff, Keeva, 1972, The magnetotelluric method in the exploration of sedimentary basins: Geophysics, v. 37, no. 1, p. 98-141.

Vozoff, Keeva, 1991, The magnetotelluric method, in Nabighian, M.N., ed., Electromagnetic Methods in Applied Geophysics, Volume 2, Application, Part B, p. 641-712.

Vozoff, Keeva, Hasegawa, H., and Ellis, R.M., 1963, Results and limitations of magnetotelluric surveys in simple geologic situations: Geophysics, v. 28, no. 5, Part I, p. 778-792.

Zonge, K.L., and Hughes, L.J., 1991, Controlled Source AudioFrequency Magnetotellurics, in Nabighian, M.N., ed., Electromagnetic Methods in Applied Geophysics, Volume 2, Application, Part B, p. 713-809. 


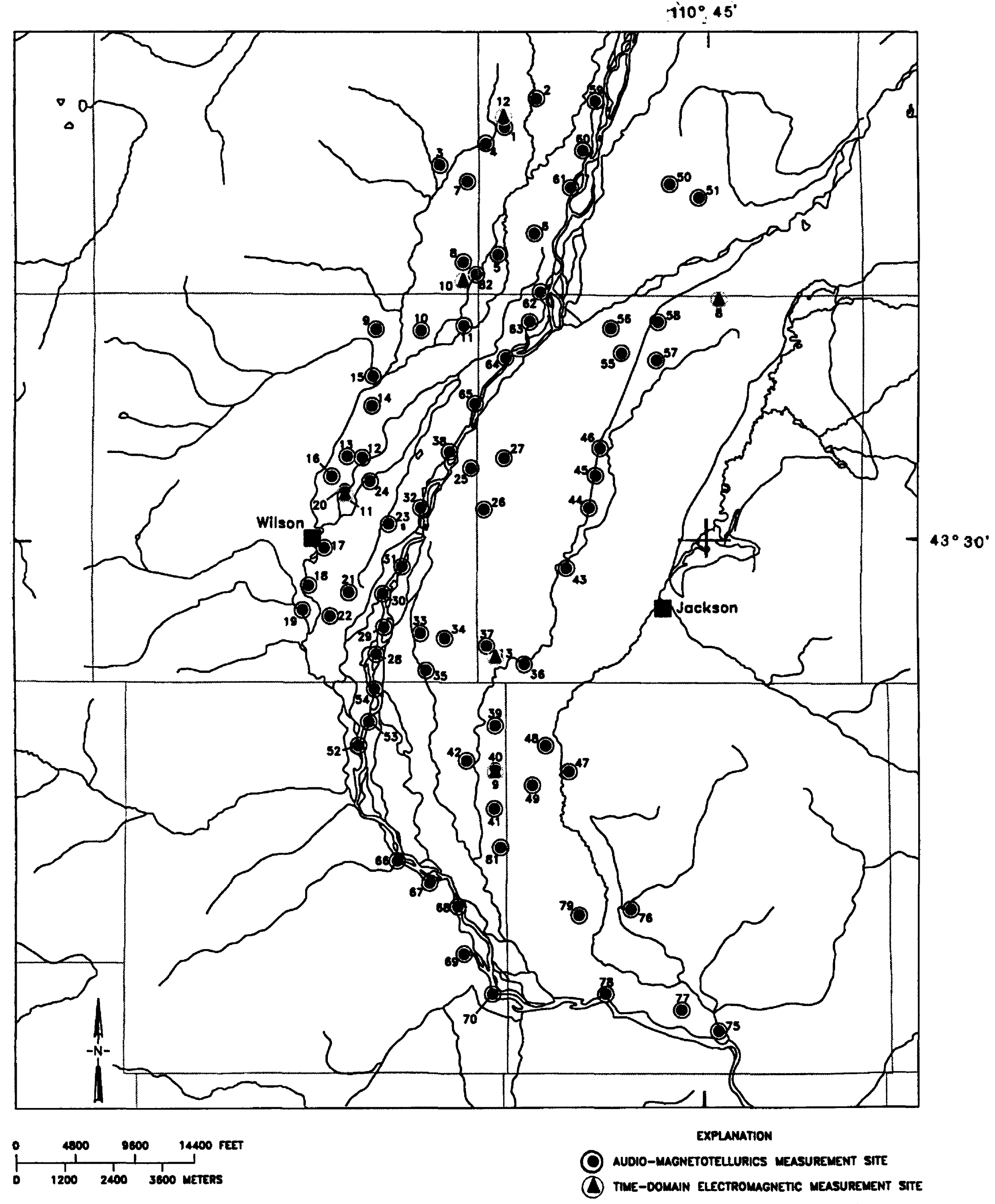

FIGURE 1. Map showlng major hydrologic feafures and audio-magnefotellurics and time-domain electromagnefic measurement site locations, Jackson Hole, Wyoming. 


\section{Appendix A}

AMT sounding data for stations recorded at Jackson Hole WY. Key to abbreviations:

Sta. ID

Station identification. Suffix "A" indicates stations repeated in Sept 1994.

Freq.

Frequency $(\mathrm{Hz})$

No Freq.

Number of frequencies recorded

Ap Res

Apparent Resistivity (Ohm-meters)

$\mathrm{N}$ Obs

Number of observations taken

Std Err

Standard error

(8)

$0=N S$

North-South E-field measurement

$\mathrm{x}=\mathrm{EW}$

East-West E-field measurement 


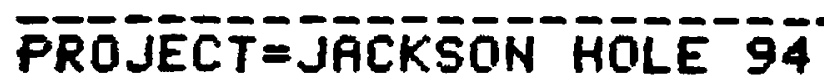

STR. ID_TCEI NS NO FREQ $=15$

FRER AP-RES N OES STD ERR

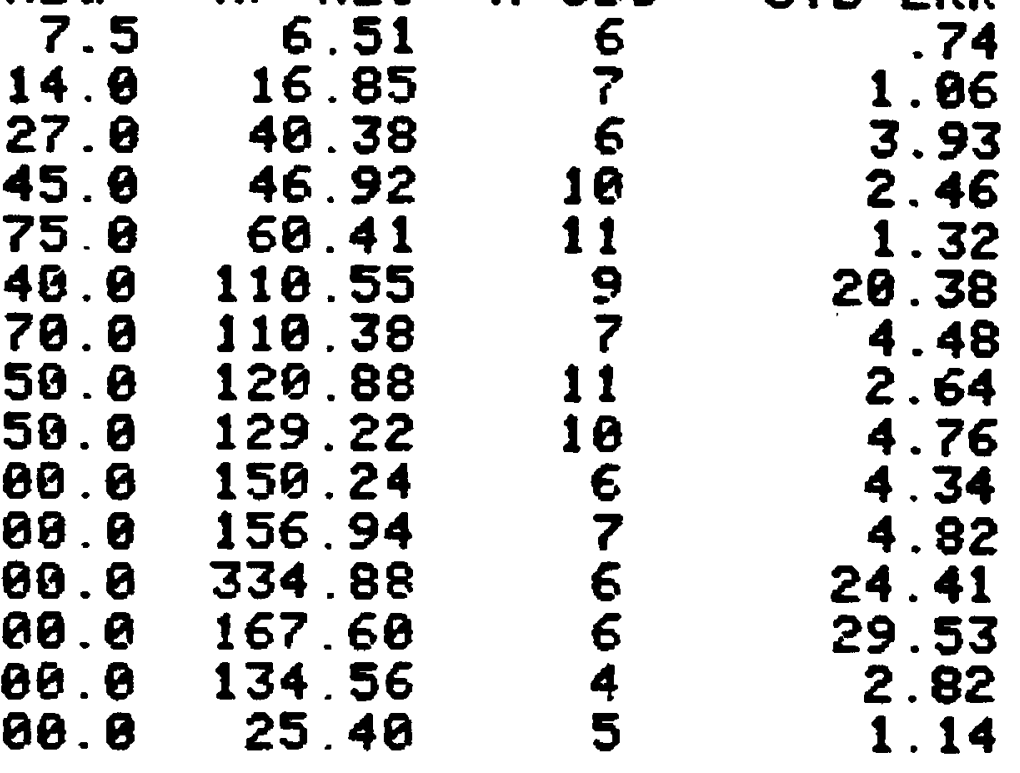

STA. ID_TCEI EN NO FREQ= ?

$\begin{array}{rrrr}\text { FREQ } & \text { AP-RES } & \text { N OQS } & \text { STO ERR } \\ 75.0 & 282.89 & 9 & 39.68 \\ 750.0 & 336.35 & 9 & 15.77 \\ 2780.0 & 92.18 & 3 & 5.63 \\ 4500.0 & 234.86 & 8 & 16.43 \\ 7500.8 & 139.32 & 19 & 3.84 \\ 14989.0 & 64.47 & 4 & .81 \\ 27000.0 & 10.50 & 6 & .30\end{array}$

PROJECTT=JACKSON HOLE 94

STA. ID_TCO2 NS NO FREQ $=11$

FREQ AP-RES N OBS STD ERR $\begin{array}{llll}4.5 & 191.49 & 6 & 5.26\end{array}$ $\begin{array}{llll}7.5 & 83.52 & 9 & 15.92\end{array}$

$14.51 .74 \quad 8 \quad 9.95$

$\begin{array}{llll}45.0 & 188.66 & 8 & 20.74\end{array}$

$140.0586 .33 \cdot 10 \quad 186.42$

$270.01152 .50 \quad$ e 196.39

$\begin{array}{llll}750.0 & 686.83 & 8 & 146.30\end{array}$

$1408.8267 .41 \quad 3 \quad 19.11$

$2708.0 \quad 284.92 \quad 2 \quad 28.15$

$7500.0 \quad 348.27 \quad 6 \quad 15.27$

$14000.0 \quad 95.31 \quad 3 \quad 2.51$

STA. ID_TCB2 EW NO FREQ $=13$

FREQ AP-RES N OBS STD ERR $\begin{array}{llll}4.5 & 93.29 & 4 & 13.56\end{array}$

$\begin{array}{llll}7.5 & 102.94 & 5 & 11.38\end{array}$

$\begin{array}{llll}14.8 & 64.99 & ? & 12.13\end{array}$

$\begin{array}{llll}27.0 & 98.71 & 11 & 6.81\end{array}$

$\begin{array}{llll}45.8 & 69.64 & 7 & 15.93\end{array}$

$75.8 \quad 148.20 \quad 6 \quad 23.61$

$\begin{array}{llll}140.0 & 290.41 & 18 & 25.42\end{array}$

$\begin{array}{llll}270.0 & 415.54 & 9 & 46.59\end{array}$

$\begin{array}{llll}450.0 & 923.09 & 10 & 53.77\end{array}$

$\begin{array}{llll}750.0 & 736.21 & 8 & 32.34\end{array}$

$\begin{array}{llll}1400.9 & 682.52 & 7 & 13.66\end{array}$

$\begin{array}{llll}4500.0 & 672.00 & 8 & 51.41\end{array}$

$\begin{array}{llll}7500.0 & 757.20 & 8 & 33.62\end{array}$
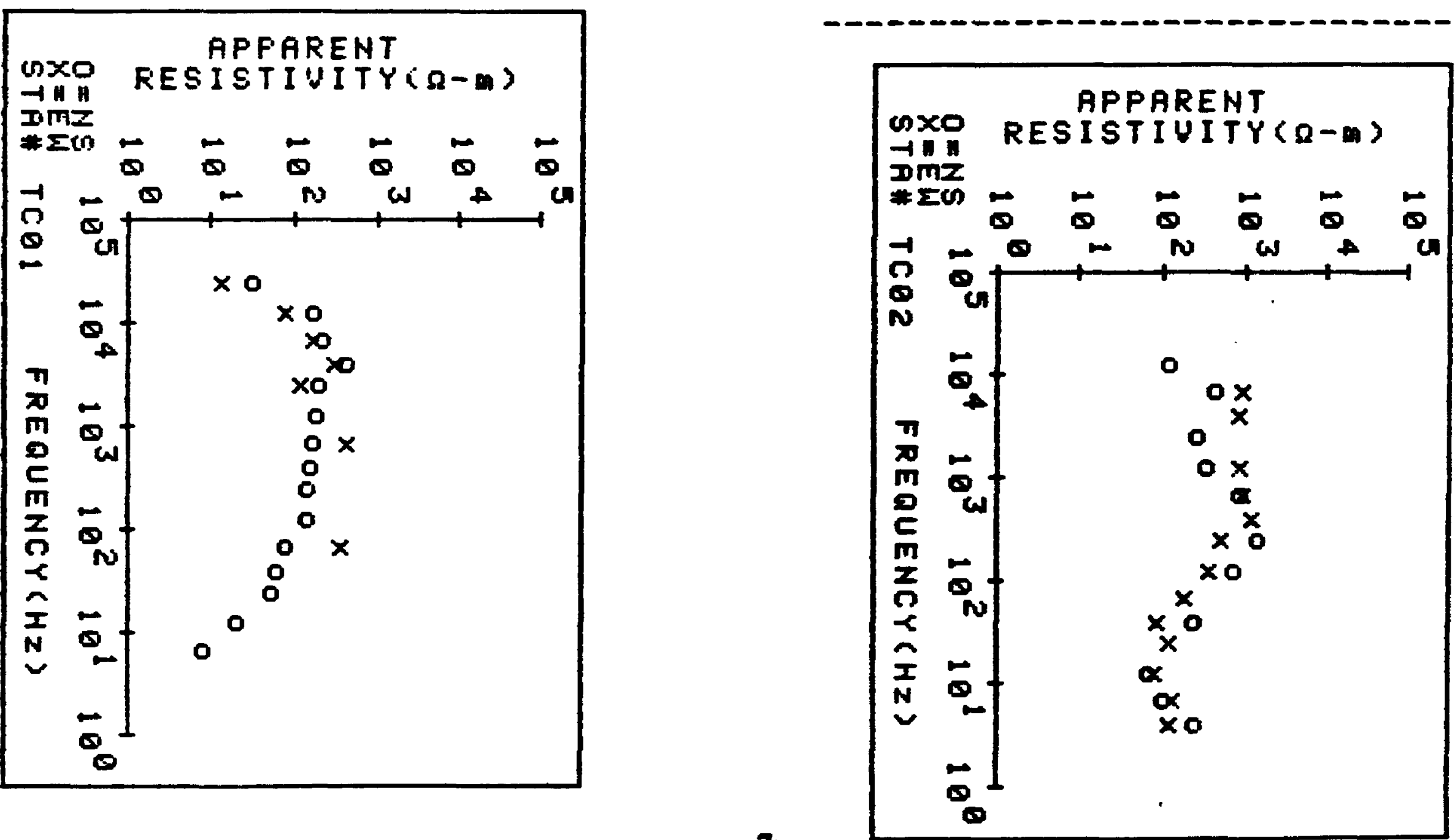


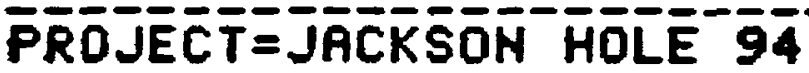

STA. ID_TCO3 NS NO FREQ $=10$

$\begin{array}{rccr}\text { FREQ } & \text { AP-RES } & N \text { OES } & \text { STD ERR } \\ 4.5 & 212.49 & 6 & 16.84 \\ 7.5 & 434.45 & 4 & 9.34 \\ 14.0 & 298.46 & 7 & 188.50 \\ 45.8 & 212.82 & 7 & 37.53 \\ 75.0 & 169.59 & 8 & 46.49 \\ 140.0 & 145.18 & 4 & 5.97 \\ 270.0 & 174.63 & 19 & 36.80 \\ 450.0 & 218.27 & 6 & 58.88 \\ 4560.0 & 207.96 & 4 & 31.70 \\ 7500.0 & 220.26 & 8 & 29.67\end{array}$

STA. ID_TCO3 EW NO FREQ= 9

$\begin{array}{rrrr}\text { FREQ } & \text { AP-RES } & N \text { OBS } & \text { STD ERR } \\ 4.5 & 9.87 & 4 & .19 \\ 7.5 & 4.57 & 5 & .35 \\ 14.0 & 4.94 & 5 & 2.67 \\ 276.0 & 2.83 & 5 & .84 \\ 450.0 & 1.79 & 6 & .86 \\ 750.0 & 2.09 & 5 & .27 \\ 4560.0 & 6.38 & 5 & .18 \\ 4606.0 & 3.12 & 3 & .06 \\ 7800.0 & 4.23 & 2 & .09\end{array}$

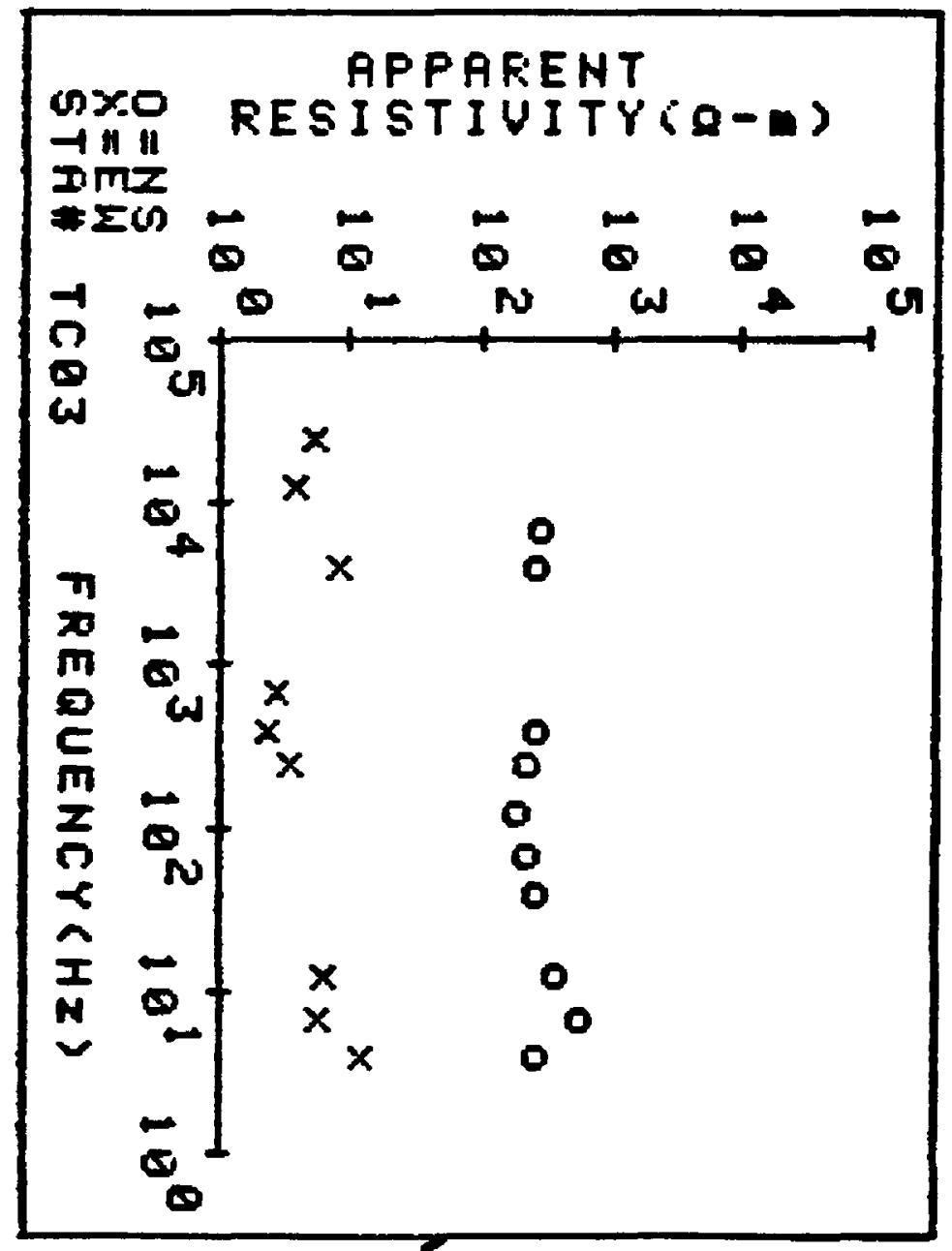

PROJE JACKSON HOLE 94

STA-IDTCO4RF ILE NAME=TC8 $4 A^{\circ}$

- - - - n

'ROJECT JACKSON HOLE $94=$

STA. ID_TCO4A NS NO FREQ= 8

FREQ AP-RES N OBS STD ERR

$7.5: 619.76 \cdots 6 \ldots 55.79$

14.0481 .00 . 7 - 116.85

$27.9606 .09 \div 615.12$

$45.9610 .28 \cdots 3.48$

$75.9 \quad 827.36 \ldots 78 \quad 28.02$

$278.0 \quad 817.26 \quad \cdots 10 \quad \ldots .68 .74$

$458.0 \quad 693.09 \quad 18 \quad \because \quad 48.24$

$1408.01539 .56 \cdot .8 \cdot 131.44$

5TA. ID_TCO4A EH NO FREQ 13 .

FREQ AP-RES N OBS STD ERR $7.5 \quad 187.16$

14.9517 .91

4501040.70

$75.0 \quad 520.44$

$140.8 \quad 314.58$

$270.0 \quad 358.78$

$450.8 \quad 291.31$

$750.8 \quad 336.46$

2700.8988 .45

4500.0668 .59

$7500.0 \quad 47.22$

.14009 .9255 .00

27000.0108 .75
10.61

100.17

17.25

16.57

12.83

42.26

28.91

39.41

91.69

23.23

1.49

6.65

29.89

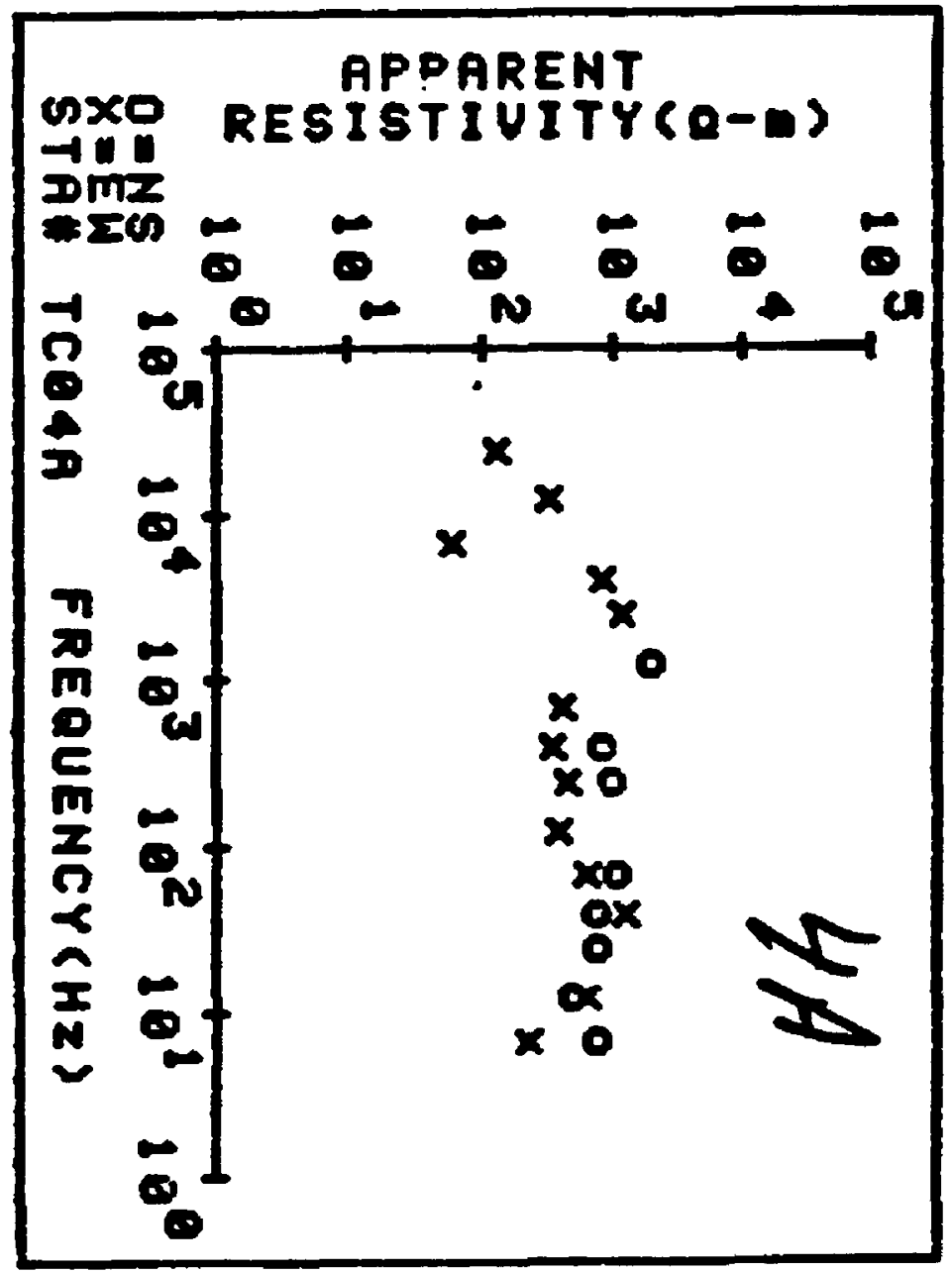


PROAJECT'JAACKSON HOLE

STA. ID_TCGS NS NO FREQ $=5$

$\begin{array}{rrrr}\text { FREO } & \text { AF-FES } & \text { N OES } & \text { STU EFP } \\ 4.5 & 15.22 & 5 & 6.39 \\ 14.9 & 45.33 & 7 & 8.53 \\ 45.9 & 128.83 & 9 & 46.59 \\ 276.9 & 284.21 & 9 & 11.52 \\ 450 . & 889.79 & 8 & 74.40\end{array}$

STA. ID_TCQS EW NO FREQ = 10

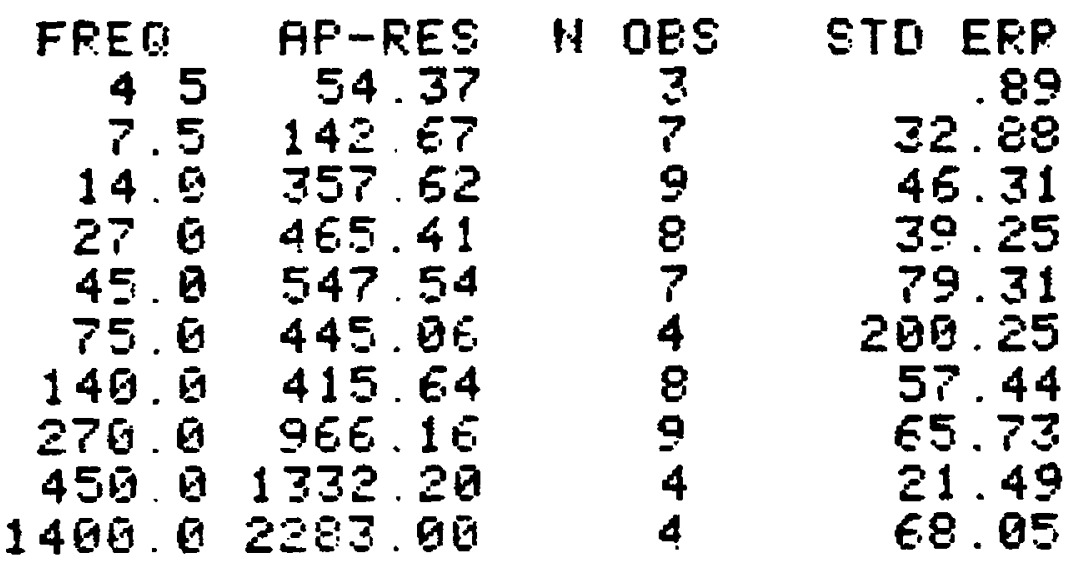

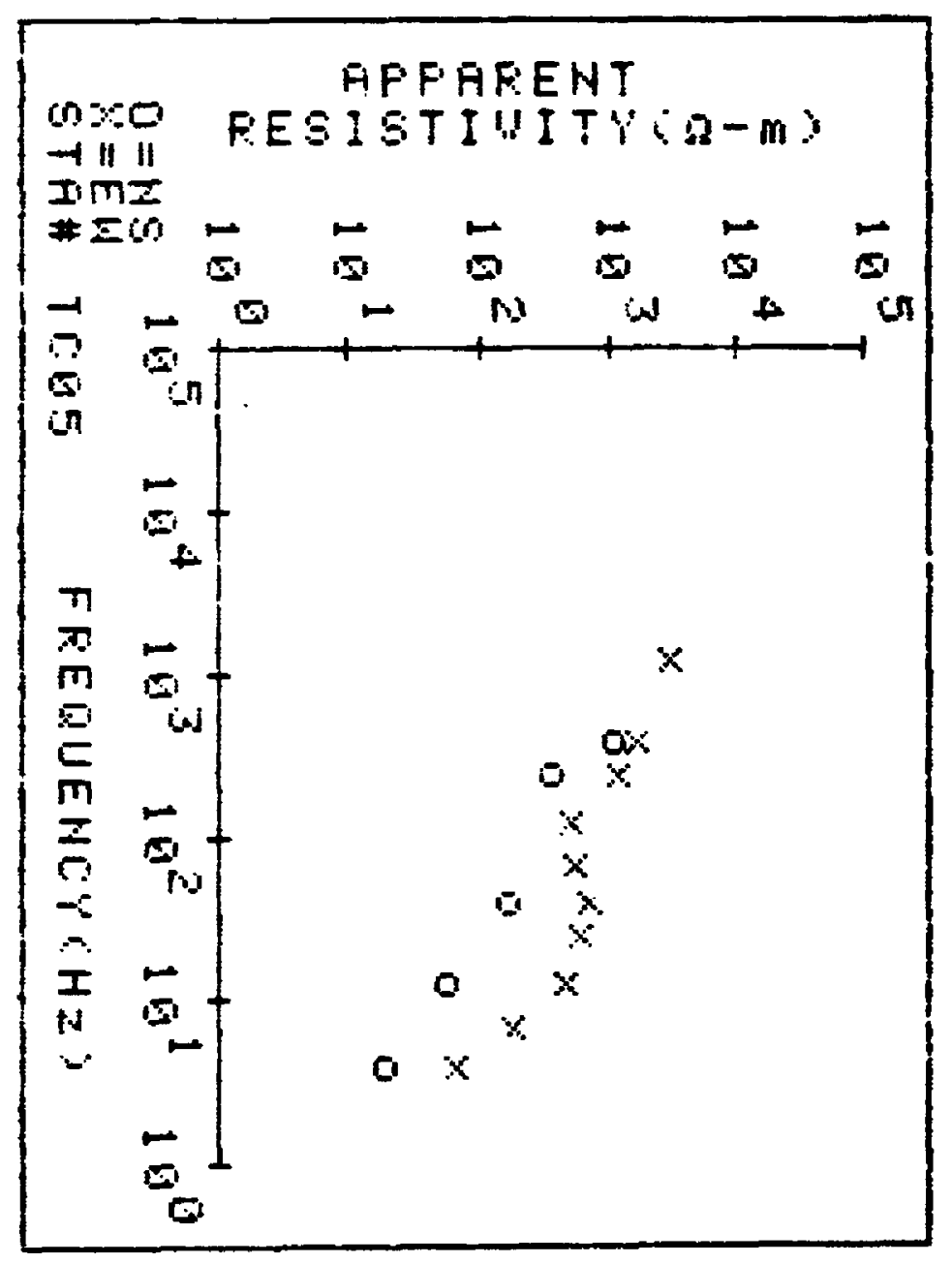

$T c-\phi 5$

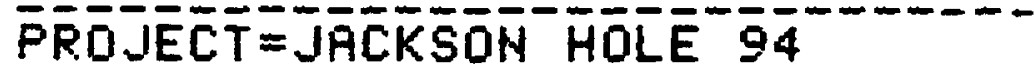

STA. ID_TCOE NS NO FFEQ $=\epsilon$

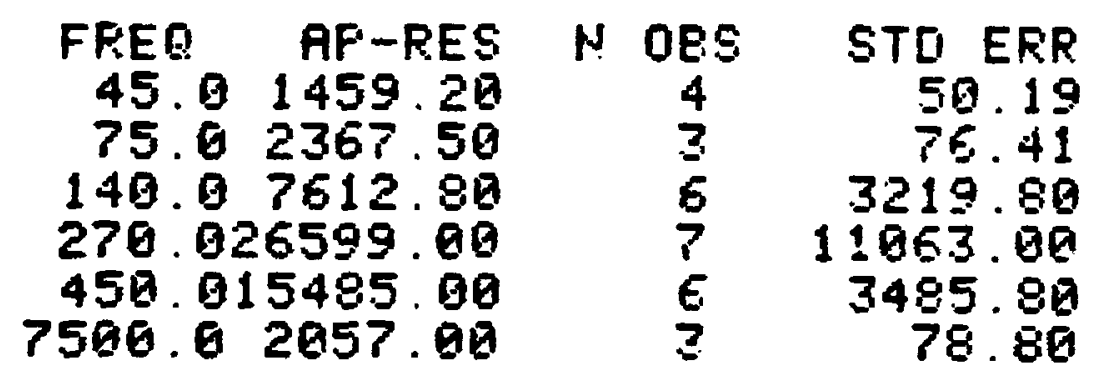

STA. ID_TCOE EW NO FREQ $=9$

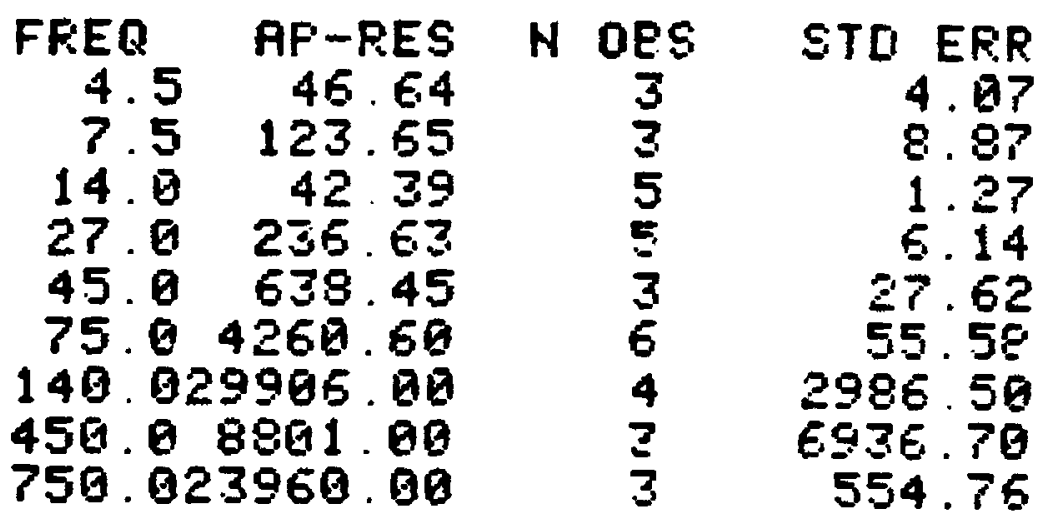

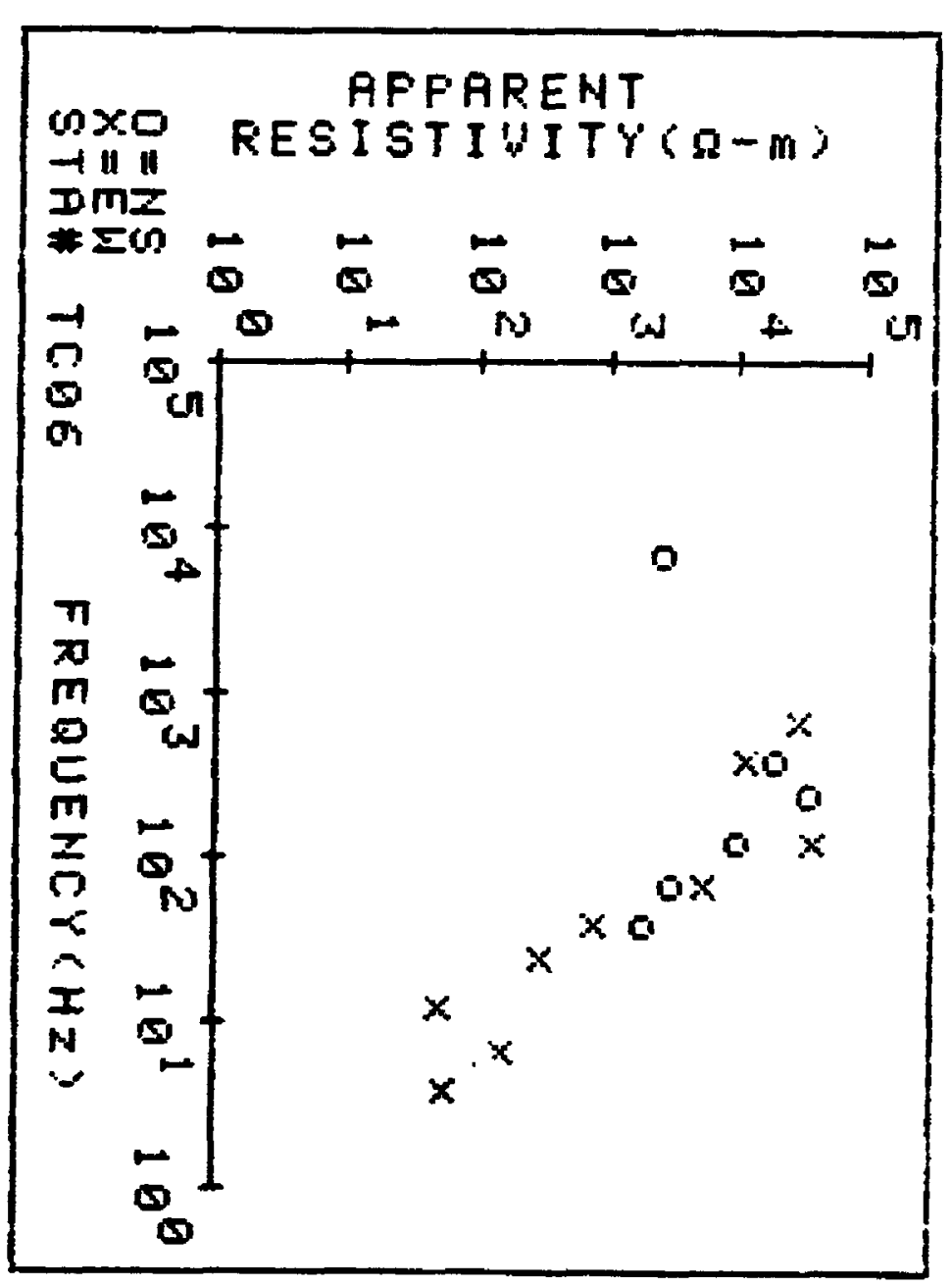

$T c-\phi b$

9 


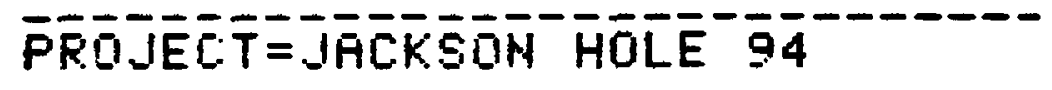

STA. ID_TCOZ NS NO FPEQ $=10$
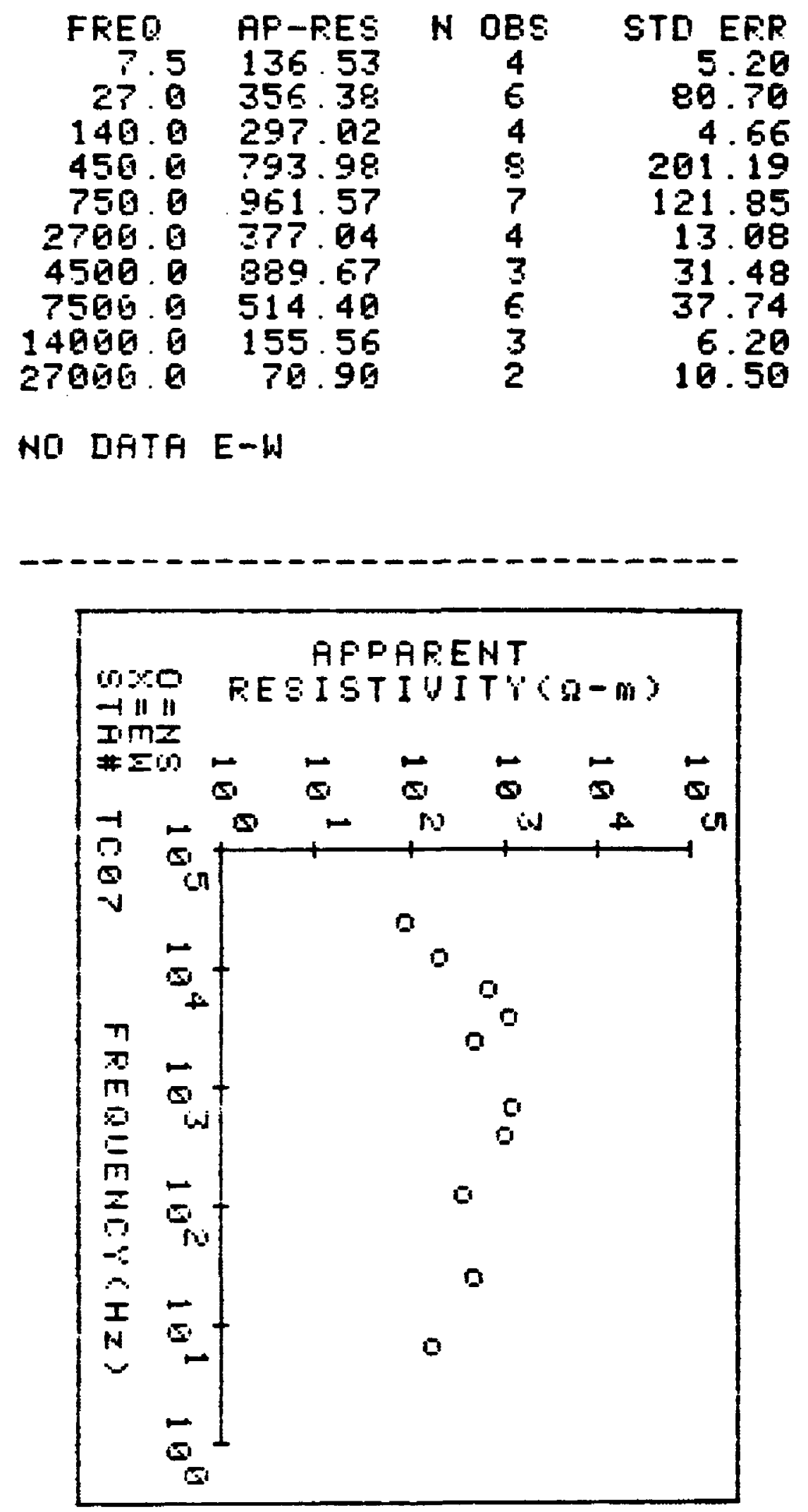

TC-OF
PROJECT JACEKSON HOLE NO DATA N-S

STA. ID_TC.OS EW HO FREQ $=4$

$$
\begin{array}{rrrr}
\text { FREQ } & \text { AF-RES } & \text { N OBS } & \text { STO EFR } \\
4.5 & 15.08 & 3 & .63 \\
4500.0 & 32.39 & 4 & 2.85 \\
7500.0 & 49.28 & 2 & 16.87 \\
4000.0 & 100.38 & .1 & 0.06
\end{array}
$$

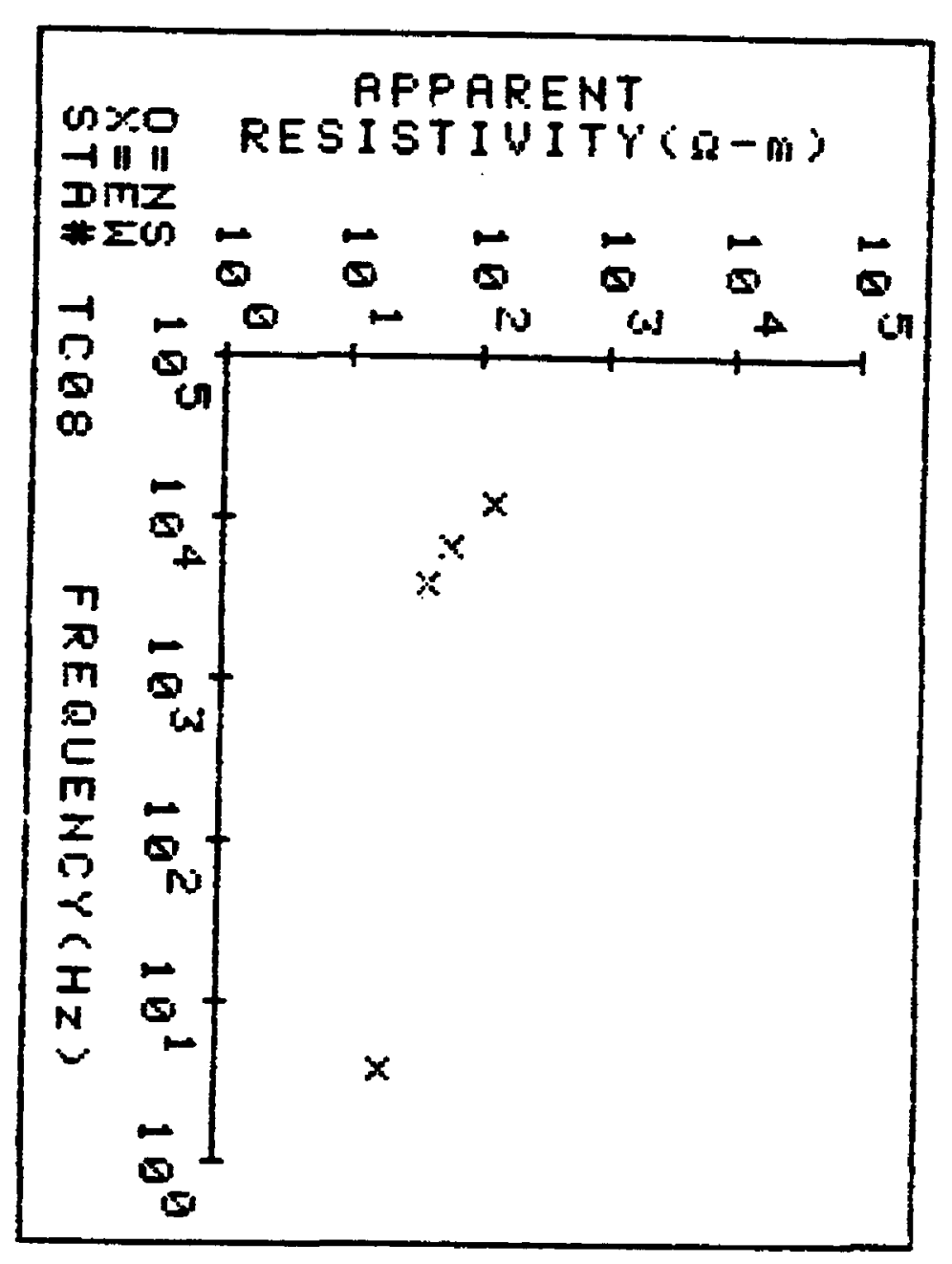

$T C-\phi 8$ 
P'ROJJECTT=JACK

STA. ID_TCO9 NS NO FREQ $=10$

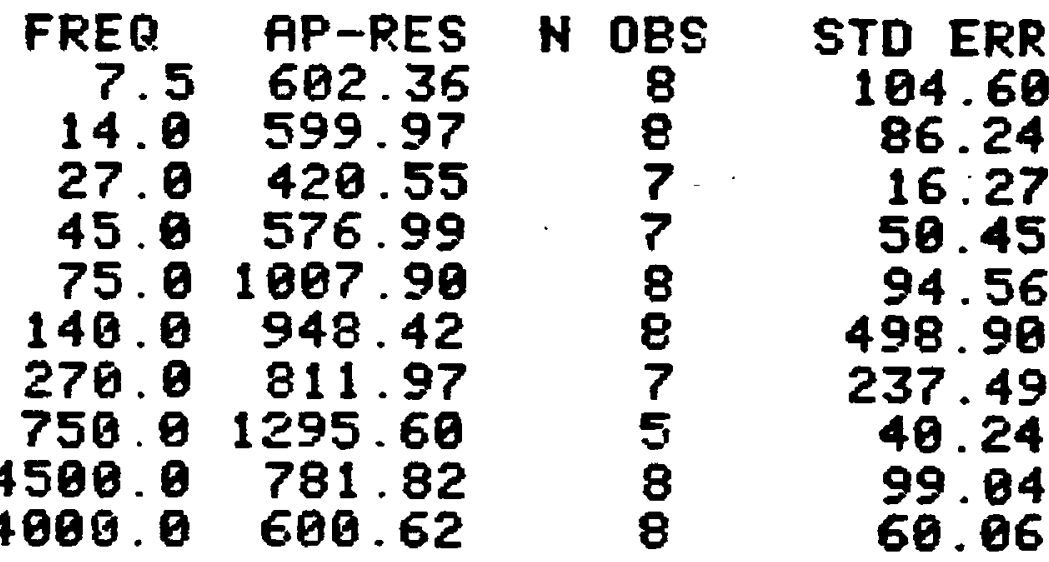

STA. ID_TCBS EH NO FREQ= 7

\begin{tabular}{|c|c|c|c|}
\hline $\begin{array}{l}\text { REQ } \\
7.5 \\
14.0 \\
27.0 \\
45.8 \\
75.0\end{array}$ & $\begin{array}{r}\text { AP-RES } \\
45.48 \\
46.60 \\
123.96 \\
173.38 \\
1429.38 \\
4487.80\end{array}$ & $\begin{array}{c}\text { N OBS } \\
4 \\
3 \\
5 \\
5 \\
7 \\
7 \\
3\end{array}$ & $\begin{array}{r}3 \\
7 \\
108 \\
18 \\
62\end{array}$ \\
\hline
\end{tabular}

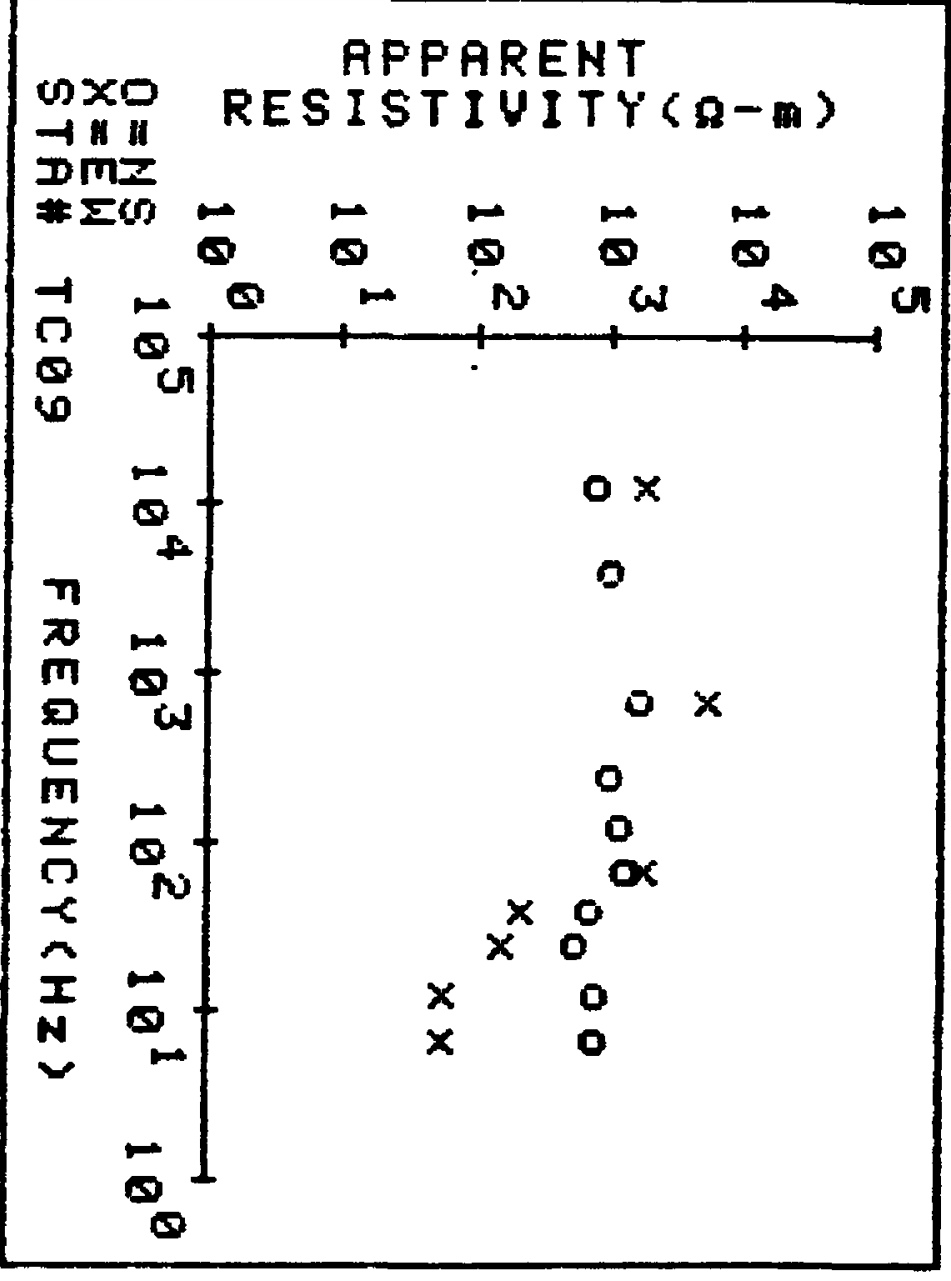

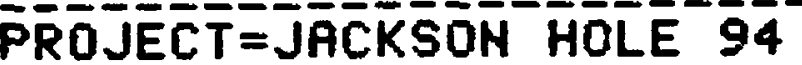

STA. ID_TCIO NS HO FREQ $=2$

FREQ AP-RES N OBS STD ERR $14.0 \quad 446.40 \quad 1 \quad 0.80$ $27.81587 .48 \quad 2 \quad 1419.00$

STA. ID_TC1O EW NO FREQ $=9$

FREQ AP-RES N OES STD ERR $\begin{array}{llll}4.5 & 883.48 & 3 & 43.24\end{array}$

$7.51006 .58 \quad 3 \quad 233.58$

$14.02434 .39 \quad 3 \quad 8.49$

$\begin{array}{llll}27.8 & 4468.70 & 4 & 117.74\end{array}$

$45.06398 .30 \quad 8 \quad 182.91$

$\begin{array}{llll}75.010437 .89 & 8 & 362.92\end{array}$

$140.017397 .80 \quad 8 \quad 969.04$

$\begin{array}{llll}450.06375 .18 & 7 & 91.97\end{array}$

$750.014270 .80 \quad 4 \quad 332.71$

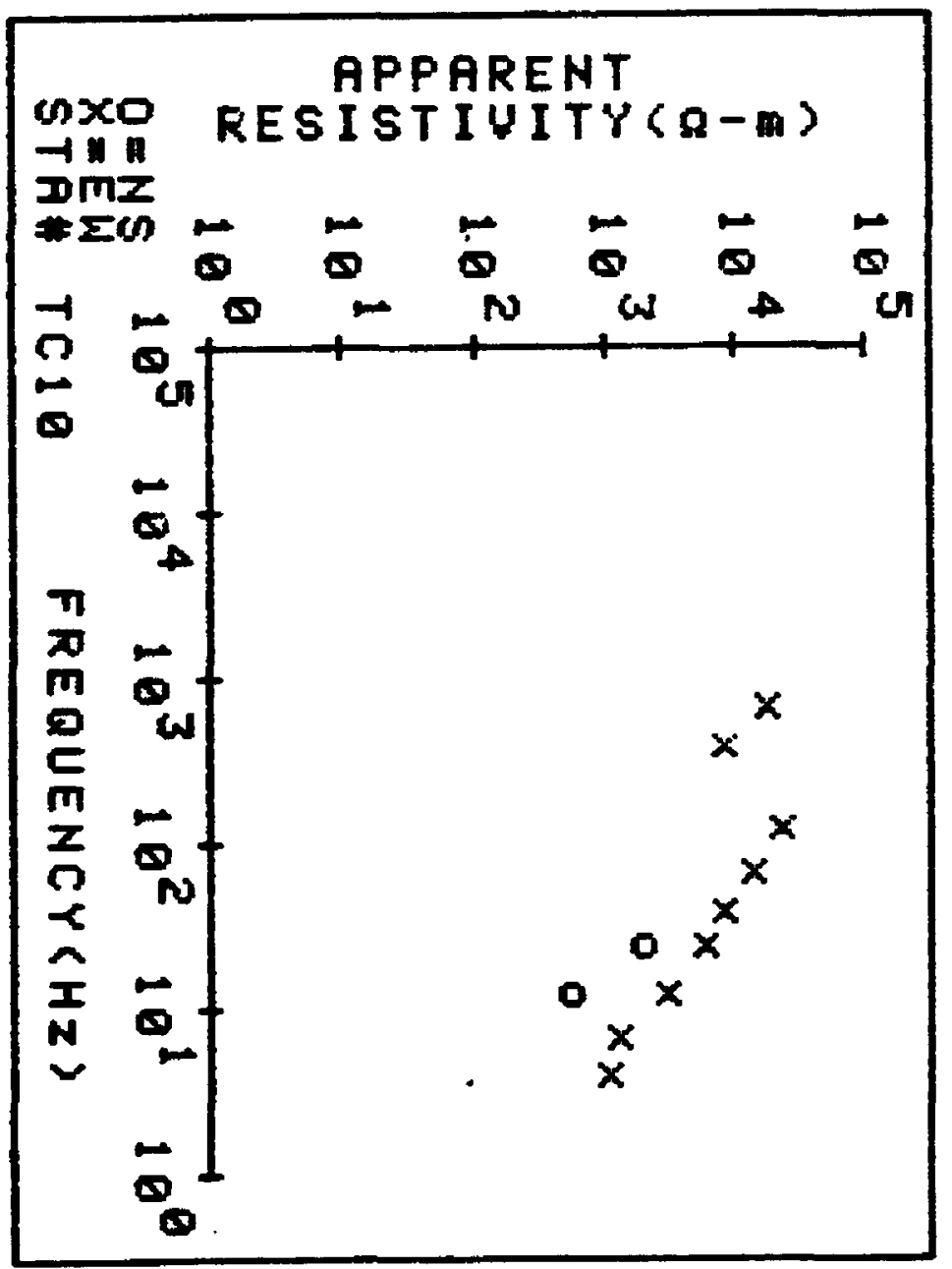


PROJECT JACKSOH HOLE 94

STA. ID_TC11 NS NO FREQ= 14

\begin{tabular}{|c|c|c|c|}
\hline $\begin{array}{r}\text { FREQ } \\
4.5 \\
7.5 \\
14.0 \\
27.0 \\
45.0 \\
75.0 \\
148.0 \\
270.0 \\
450.0 \\
750.0\end{array}$ & $\begin{array}{l}\text { AP-RES } \\
141.78 \\
163.86 \\
106.92 \\
259.54 \\
228.77 \\
549.35 \\
333.55 \\
283.65 \\
336.85 \\
465.37 \\
198.41 \\
335.89 \\
417.10 \\
114.33\end{array}$ & $\begin{array}{c}\text { N OBS } \\
3 \\
6 \\
7 \\
8 \\
8 \\
8 \\
6 \\
6 \\
8 \\
8 \\
8 \\
9 \\
3 \\
3 \\
3 \\
3 \\
3\end{array}$ & $\begin{array}{r}\text { STD ERR } \\
2.42 \\
10.03 \\
10.17 \\
35.38 \\
36.83 \\
23.36 \\
35.74 \\
19.16 \\
31.63 \\
65.26 \\
7.29 \\
108.54 \\
37.96 \\
3.38\end{array}$ \\
\hline
\end{tabular}

STA. ID_TCII EN NO FREQ $=18$

FREQ AP-RES N OBS STD ERR

$7.5 \quad 20.41$

$14.0 \quad 37.26$

75.0187 .68

140.8166 .75

270.0287 .12

$450.0 \quad 24.57$

$750.0 \quad 83.86$

$4500.0 \quad 113.13$

$7500.0 \quad 217.65$

14000.65 .88

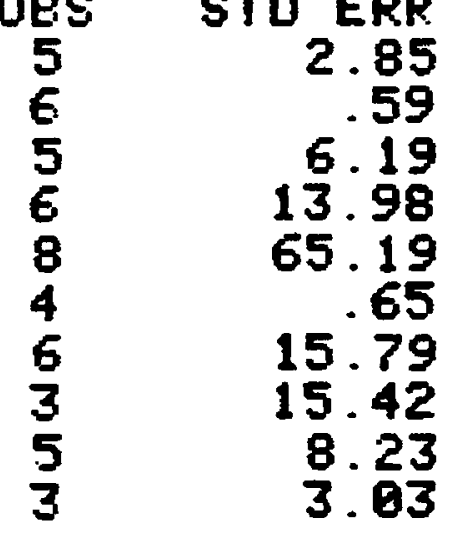

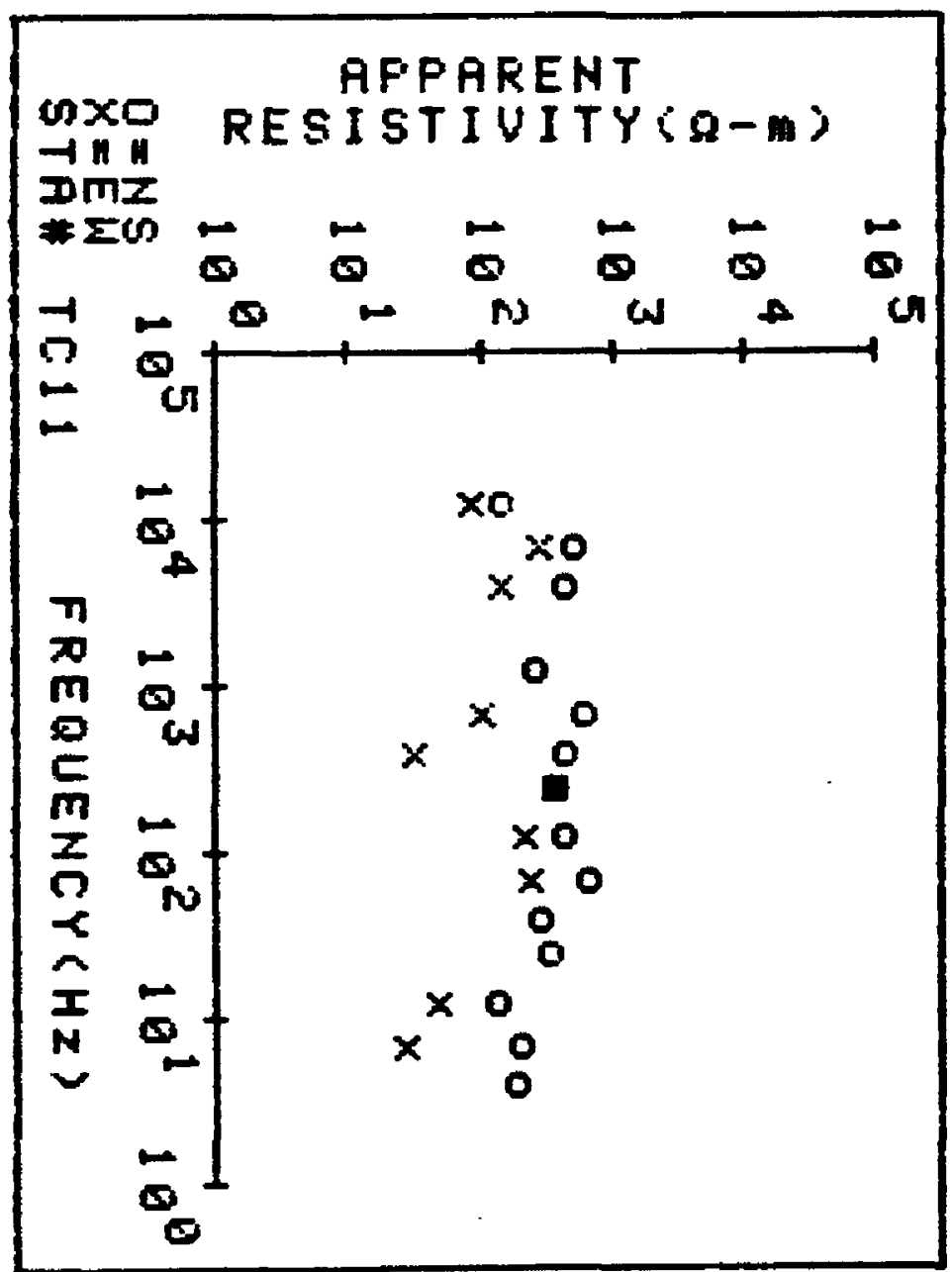

TC-II

PROJECT $=\overline{J A} \bar{C} \bar{S} \bar{O} \overline{\mathrm{N}}$ HOLE $9 \overline{9}$

STA. ID_TC12 HS NO FREQ $=5$

FREQ AP-RES $N$ OES STD ERR

$4.51178 .90 \quad 6 \quad 443.80$

$7.51173 .00 \quad 8 \quad 66.60$

$\begin{array}{llll}14.0 & 2363.50 & 7 & 70.90\end{array}$

$27.9 \quad 744.19 \quad 3 \quad 39.20$

$\begin{array}{llll}450.0 & 810.00 & 4 & 41.80\end{array}$

STA. ID_TC12 EH NO FREQ $=4$

FREQ AP-RES $N$ OES STD ERR

$4.5 \quad 524.50$

$7.5 \quad 922.00$

14.01748 .20

$27.8 \quad 619.90$
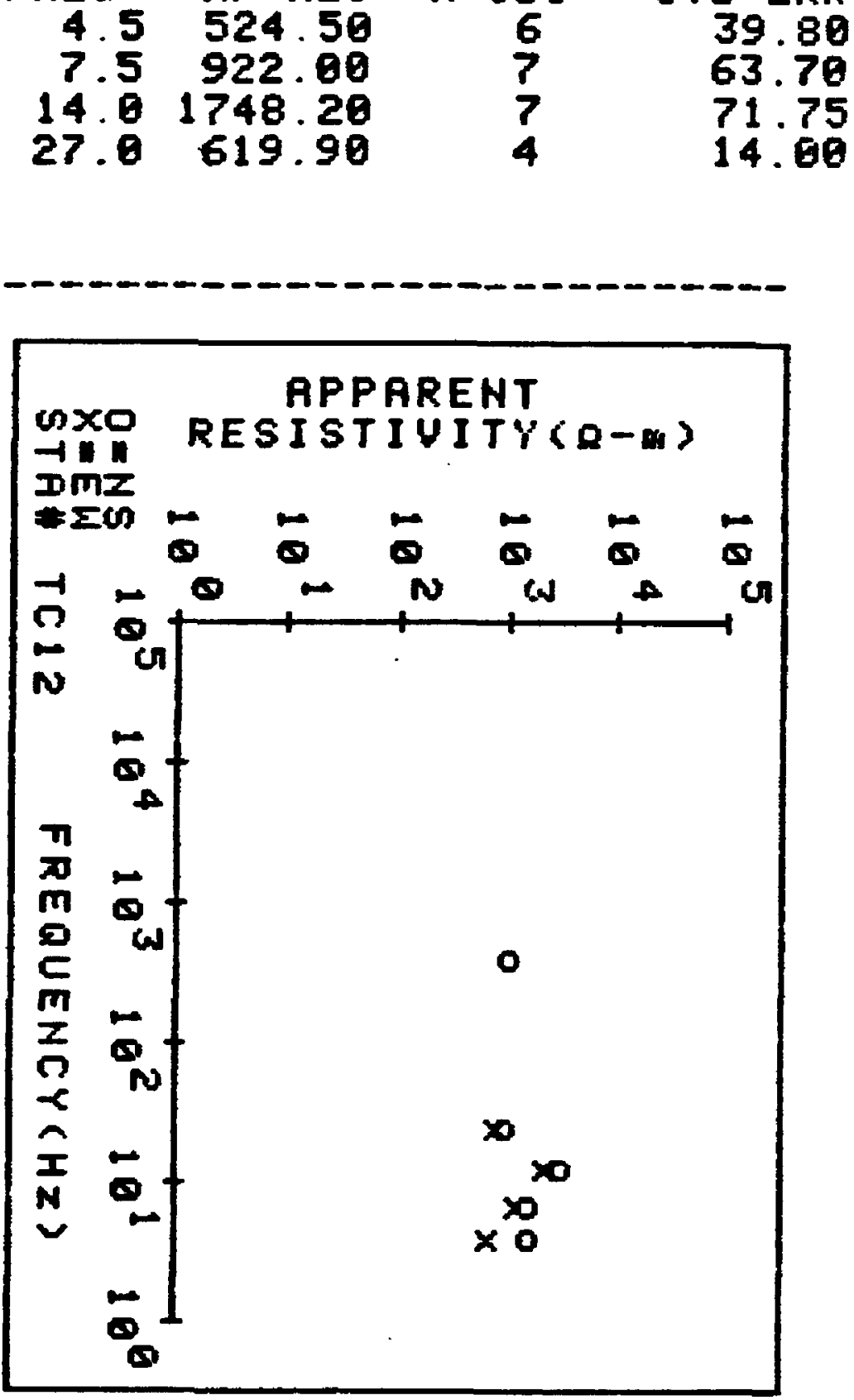
PROJ= JACKSON HOLE 94

STA-IDTC $13 F$ ILE NAME $=T_{C} 13$

PROJJECT =JACK TOON HOLE 94

STA. ID_TC13 NS NO FREQ $=12$

\begin{tabular}{|c|c|c|c|}
\hline $\begin{array}{r}\text { FREQ } \\
4.5 \\
7.5 \\
14.0 \\
27.0 \\
45.0 \\
75.0 \\
140.0 \\
756.0 \\
790.0 \\
509.0 \\
000.0 \\
000.0\end{array}$ & $\begin{array}{r}\text { AP-RES } \\
173.98 \\
191.65 \\
261.53 \\
317.09 \\
675.06 \\
440.28 \\
360.76 \\
489.33 \\
762.55 \\
567.31 \\
51.55 \\
66.59\end{array}$ & $\begin{array}{c}\text { N OBS } \\
4 \\
6 \\
6 \\
9 \\
6 \\
19 \\
9 \\
7 \\
4 \\
7 \\
3 \\
1\end{array}$ & $\begin{array}{r}\text { STD ERR } \\
14.83 \\
23.38 \\
38.54 \\
68.64 \\
141.43 \\
94.36 \\
29.16 \\
36.31 \\
132.17 \\
53.99 \\
2.77 \\
0.06\end{array}$ \\
\hline
\end{tabular}

STA. ID_TC13 EW NO FREQ $=6$

$\begin{array}{rrcr}\text { FREQ } & \text { AP-RES } & N \text { OBS } & \text { STD ERR } \\ 4.5 & 11.69 & 4 & .54 \\ 7.5 & 17.96 & 4 & .63 \\ 14.0 & 92.07 & 5 & 1.64 \\ 27.6 & 154.87 & 5 & 3.37 \\ 459.0 & 195.31 & 3 & 5.10 \\ 2796.0 & 179.64 & 3 & 27.79\end{array}$

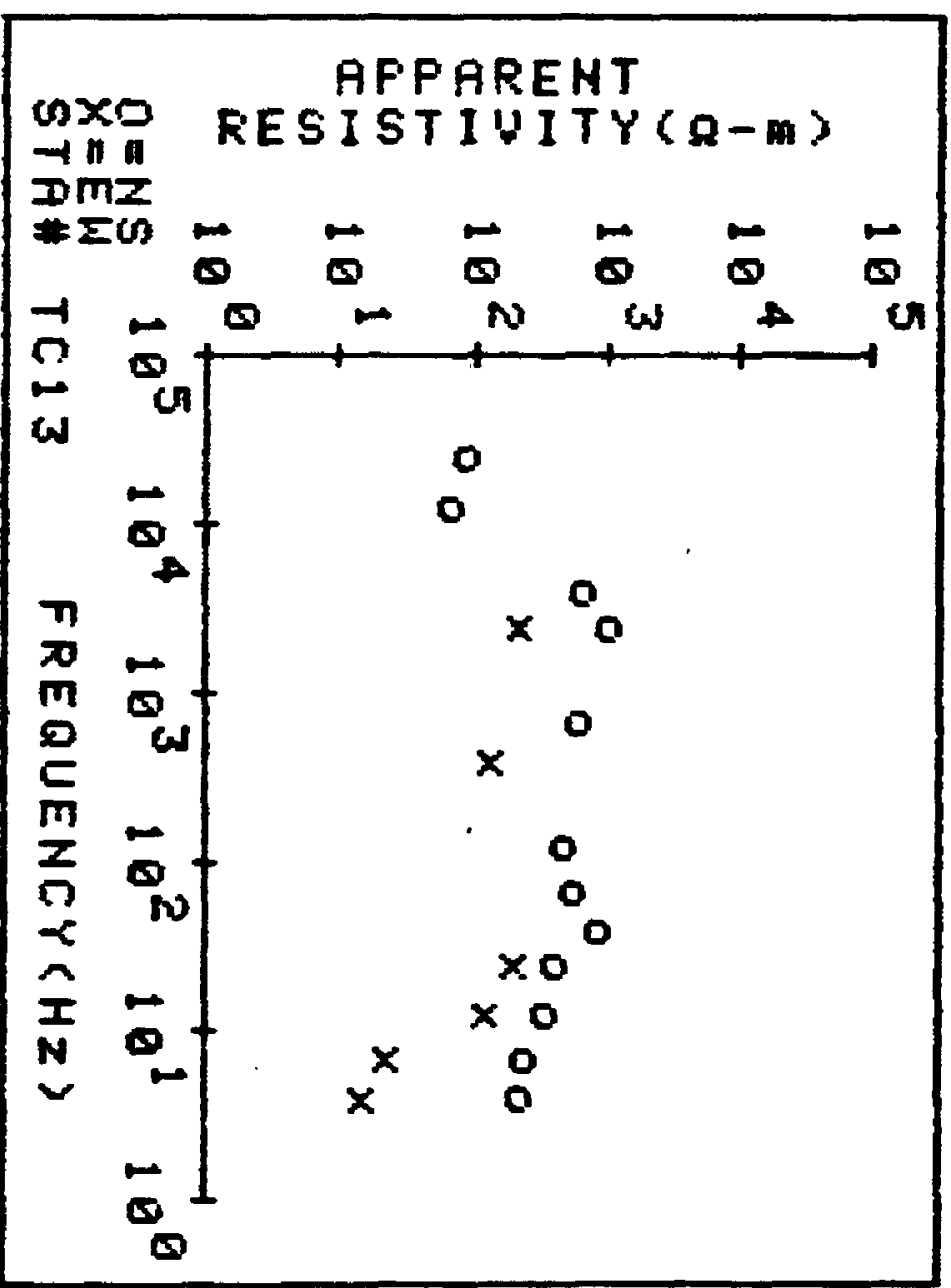

P'ROJJECT=JĀCKSOAN HOLE 94

NO DATA $\mathrm{N}-\mathrm{S}$

STA. ID_TC14 EH HO FREQ $=11$

FREQ AP-RES N OES STD ERR

$\begin{array}{llll}4.5 & 137.94 & 3 & 69.55\end{array}$

$\begin{array}{llll}7.5 & 342.89 & 7 & 49.40\end{array}$

$\begin{array}{llll}14.6 & 222.94 & 7 & 43.05\end{array}$

$27.0 \quad 426.48 \quad 8 \quad 84.14$

$\begin{array}{llll}45.8 & 483.93 & 6 & 40.37\end{array}$

$75.0 \quad 475.41 \quad 5 \quad 193.11$

$\begin{array}{llll}270.0 & 579.68 & 7 & 87.51\end{array}$

$\begin{array}{llll}4580.0 & 989.66 & 6 & 144.73\end{array}$

$\begin{array}{llll}7500.0 & 449.15 & 7 & 29.45\end{array}$

$14000.6 \quad 739.53 \quad 3 \quad 53.03$

$\begin{array}{llll}27000.0 & 172.67 & 3 & 40.69\end{array}$

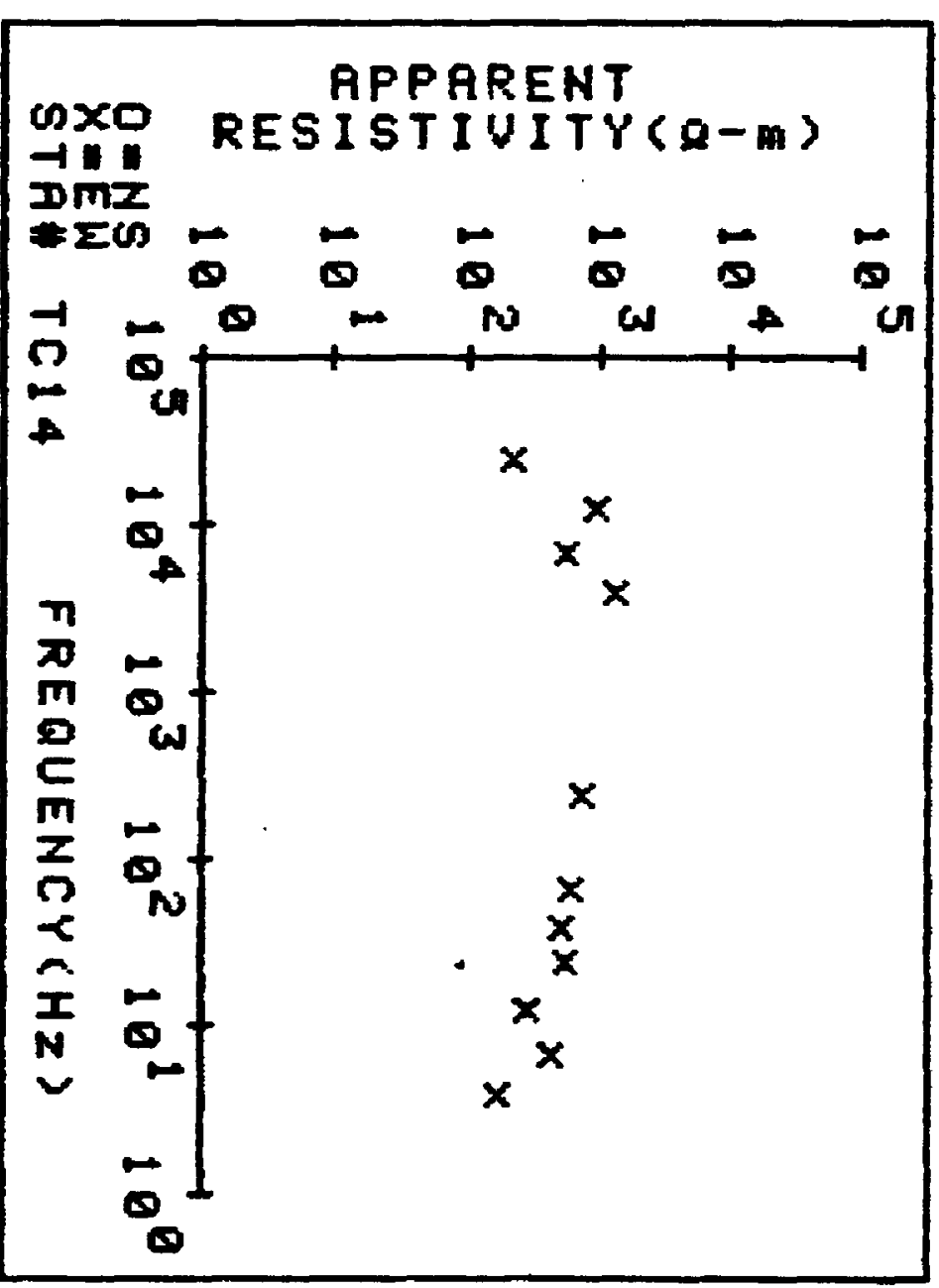


PROJ= JACKSOH HOLE 94

STA-IDTC15FILE NAME $=$ TC 15

P'ROJJECT=JĀCKSONN HOLE

STA. ID_TC15 NS NO FREQ $=5$

FREQ AP-RES N OBS STD ERR

$\begin{array}{llll}7.5 & 79.76 & 4 & 25.06\end{array}$

$\begin{array}{llll}14.0 & 63.56 & 4 & 37.68\end{array}$

$\begin{array}{llll}27.0 & 972.12 & 7 & 430.86\end{array}$

$\begin{array}{llll}45.8 & 194.21 & 4 & 369.31\end{array}$

$27000.0 \quad 32.92 \quad 2 \quad 10.97$

STA. ID_TC15 EW NO FREQ $=8$

$\begin{array}{rrrr}\text { FREQ } & \text { AP-RES } & \text { N OBS } & \text { STD ERR } \\ 4.5 & 378.14 & 7 & 97.23 \\ 7.5 & 445.91 & 5 & 79.78 \\ 14.6 & 669.20 & 8 & 162.50 \\ 27.0 & 241.35 & 9 & 66.43 \\ 45.0 & 216.32 & 9 & 372.60 \\ 75.0 & 817.03 & 9 & 1002.40 \\ 750.0 & 1005.90 & 6 & 221.59 \\ 2709.0 & 1301.70 & 2 & 424.05\end{array}$

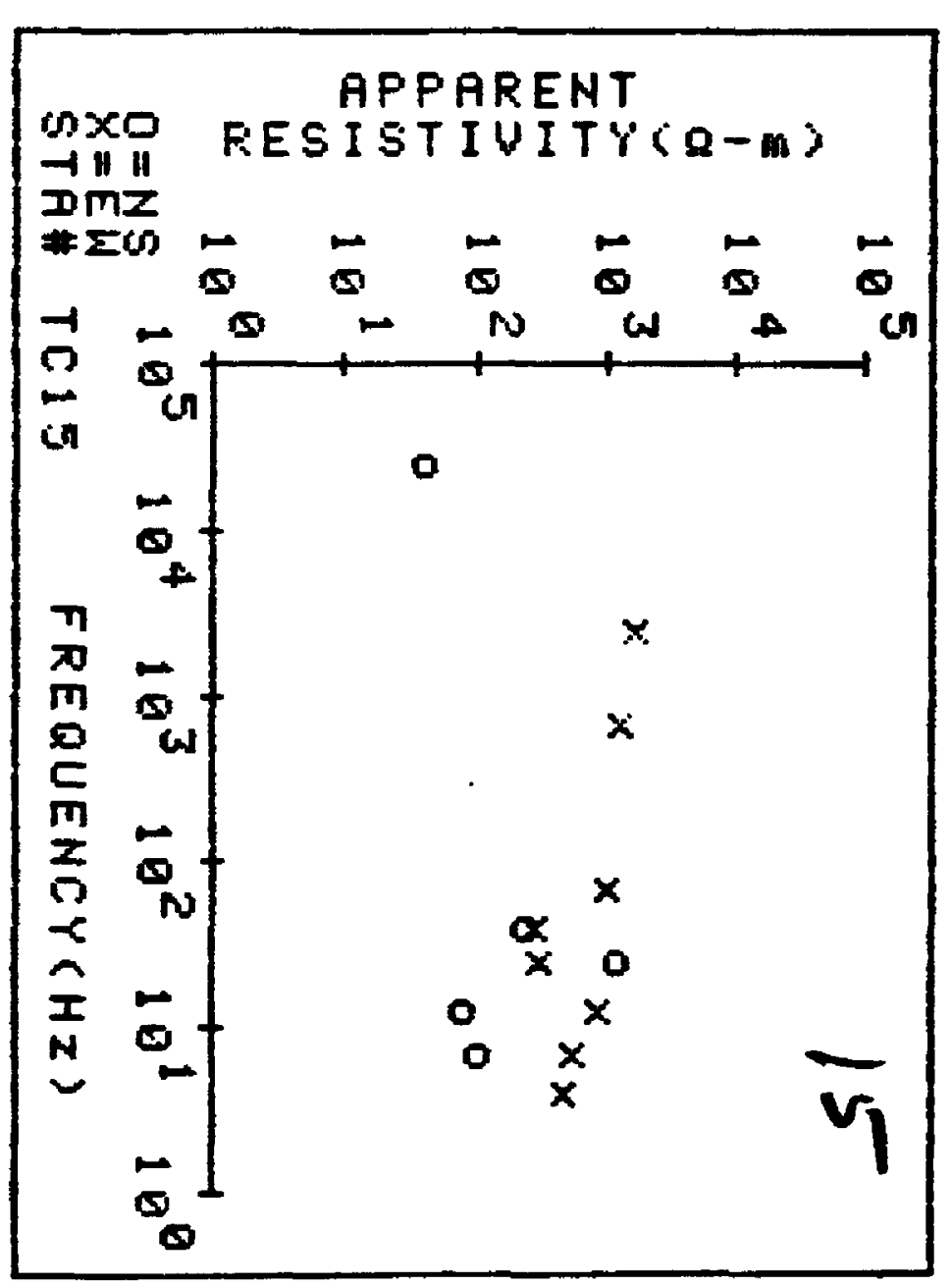

PROJ $=$ JACKSON HOLE 94

STA-IDTC16FILE NAME $=$ TC.16

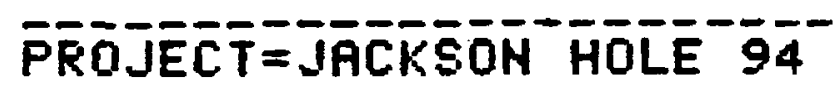

STA. ID_TC16 NS NO FREQ $=8$

FREQ AP-RES N OES STD ERR

$\begin{array}{llll}7.5 & 68.99 & 1 & 0.00\end{array}$

$\begin{array}{llll}14.0 & 126.84 & 4 & 8.30\end{array}$

$\begin{array}{llll}27.0 & 306.90 & 5 & 63.60\end{array}$

$45.0 \quad 157.40 \quad 4 \quad 5.34$

$\begin{array}{llll}75.8 & 104.26 & 4 & 3.06\end{array}$

$\begin{array}{llll}450.8 & 207.91 & 3 & 14.89\end{array}$

$\begin{array}{llll}4500.0 & 396.39 & 6 & 97.24\end{array}$

$\begin{array}{llll}7500.0 & 18.69 & 4 & .59\end{array}$

STA. ID_TCIG EW NO FREQ $=8$

FREQ AP-RES N OBS STD ERR.

$\begin{array}{lllll}45.0 & 6347 & 3 & 1.06\end{array}$

$\begin{array}{llll}75.0 & 186.83 & 3 & \end{array} .37$

$\begin{array}{llll}146.0 & 96.63 & 3 & 5.72\end{array}$

$\begin{array}{llll}270.0 & 230.74 & 3 & 5.21\end{array}$

$\begin{array}{llll}450.0 & 41.09 & 3 & .32\end{array}$

$\begin{array}{rrrr}4509.0 & 214.41 & 4 & 7.48\end{array}$

$\begin{array}{rrrr}7500.0 & 25.05 & 5 & .80 \\ 14000.0 & 40.87 & 3 & 1.99\end{array}$ 
STA ID_tc17A NS no FreQ 14 . FREQ AP-RES N OBS " ETO ERR $\therefore: 4.52101 .30$. : 4 744.51 $77.53198 .79 \quad 7.1443 .20$ $\because-.27 .06786 .69 \cdots 19 \because 1953.30$ $\because 45.86664 .79 \quad 15.967 .25$ $148.02444 .68 \quad 28$. 421.15 $\therefore 278.8805 .09 \cdot 23.184 .08$ $\begin{array}{llll}450.8 & 877.42 \quad 23 & 2 & 114.81\end{array}$ $\begin{array}{ll}758.0978 .58 \therefore 23 & 120.36\end{array}$ $1400.8582 .57 \div 12 \div 190.60$ $2780.9682 .65 \quad 19 \cdots 182.21$ $4500.0^{\circ} 243.55 \cdot 22 \cdots-48.99$ $7500.6 \quad 146.86 \cdots 18 . \cdots-31.69$ $14009.8 \quad 273.56 \quad 15 \therefore 91.05$ $27080.0 \cdot 18.71-23 \therefore \ldots 5.02$

sTR." ID_TCLTA EU NO FREQ 16

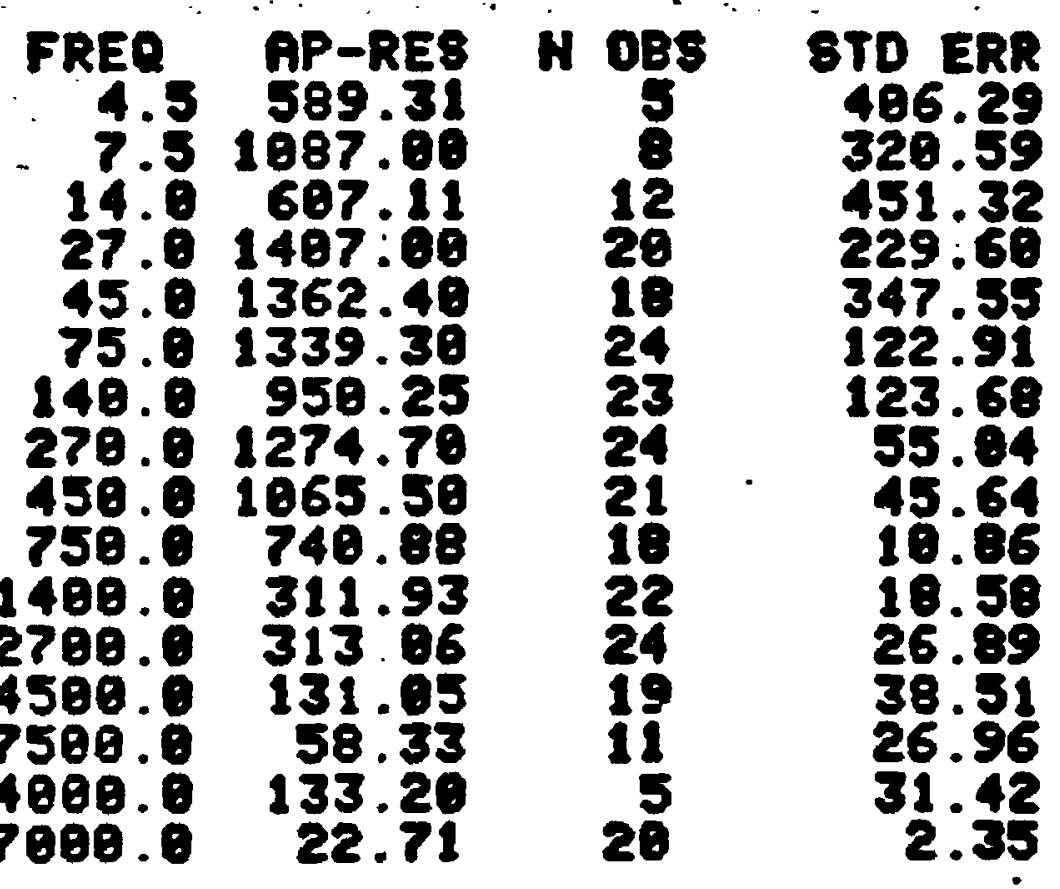

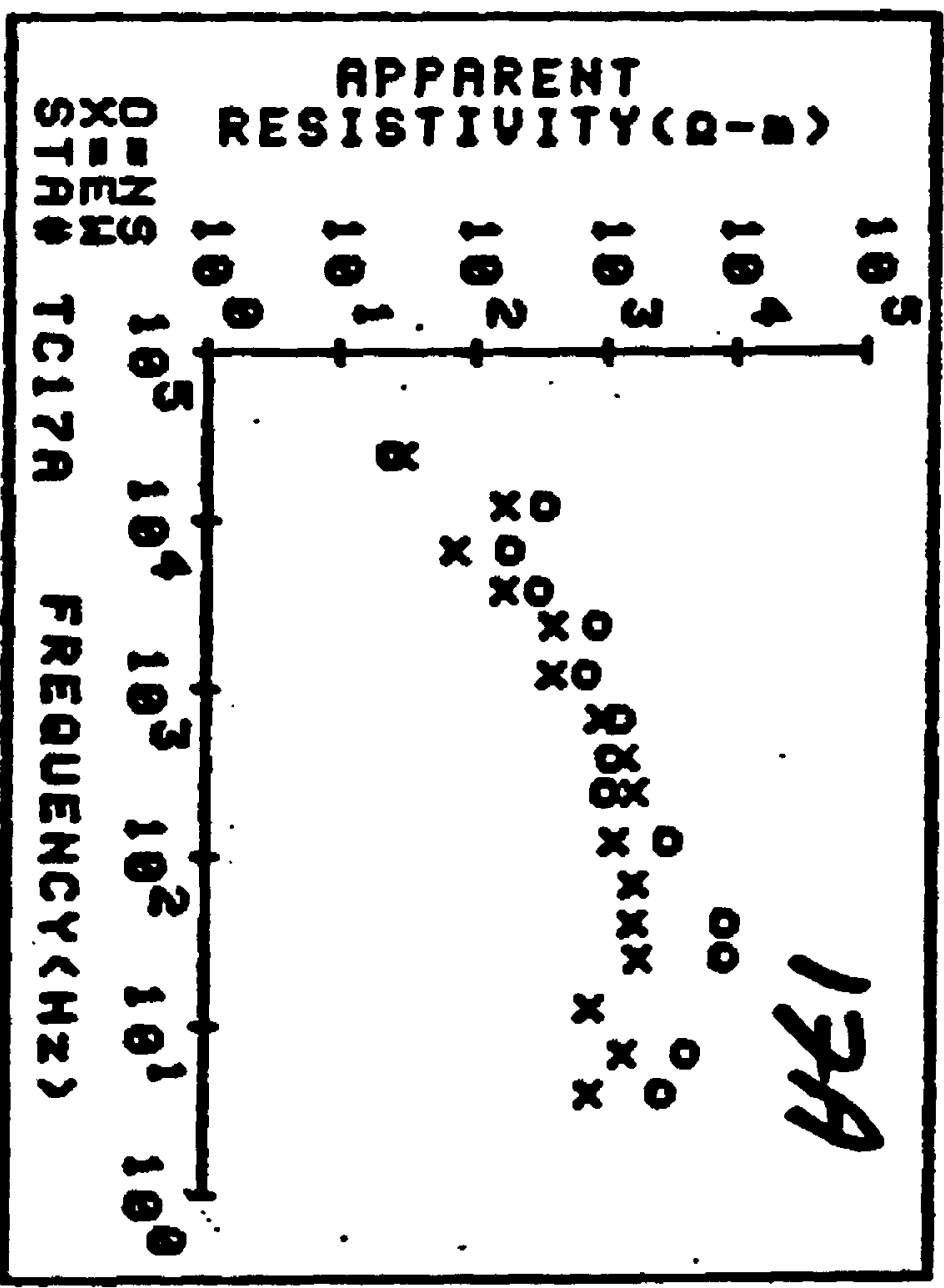

\begin{tabular}{|c|c|c|c|}
\hline TA. ID_ & TC18 NS & NO FF & 4 \\
\hline $\begin{array}{r}\text { FREQ } \\
27.8 \\
75.8 \\
270.8 \\
450.0\end{array}$ & $\begin{array}{r}\text { AP-RES } \\
46.28 \\
7.93 \\
104.78 \\
410.78\end{array}$ & $\begin{array}{c}\text { N OBS } \\
6 \\
3 \\
3 \\
6\end{array}$ & $\begin{array}{r}\text { STO } E F \\
93.2 \\
173.2\end{array}$ \\
\hline TA. I & $190=4$ & NO FRE & $=6$ \\
\hline $\begin{array}{r}\text { FREQ } \\
27.0 \\
45.0 \\
75.0 \\
249.9 \\
450.0 \\
2700.0\end{array}$ & $\begin{array}{r}\text { AP-RES } \\
14.58 \\
10.48 \\
10.11 \\
13.33 \\
100.11 \\
158.48\end{array}$ & $\begin{array}{c}N \text { ogs } \\
3 \\
3 \\
3 \\
3 \\
3 \\
3\end{array}$ & $\begin{array}{r}\text { STD E } \\
3 . \\
4 \\
4 \\
1 .\end{array}$ \\
\hline
\end{tabular}
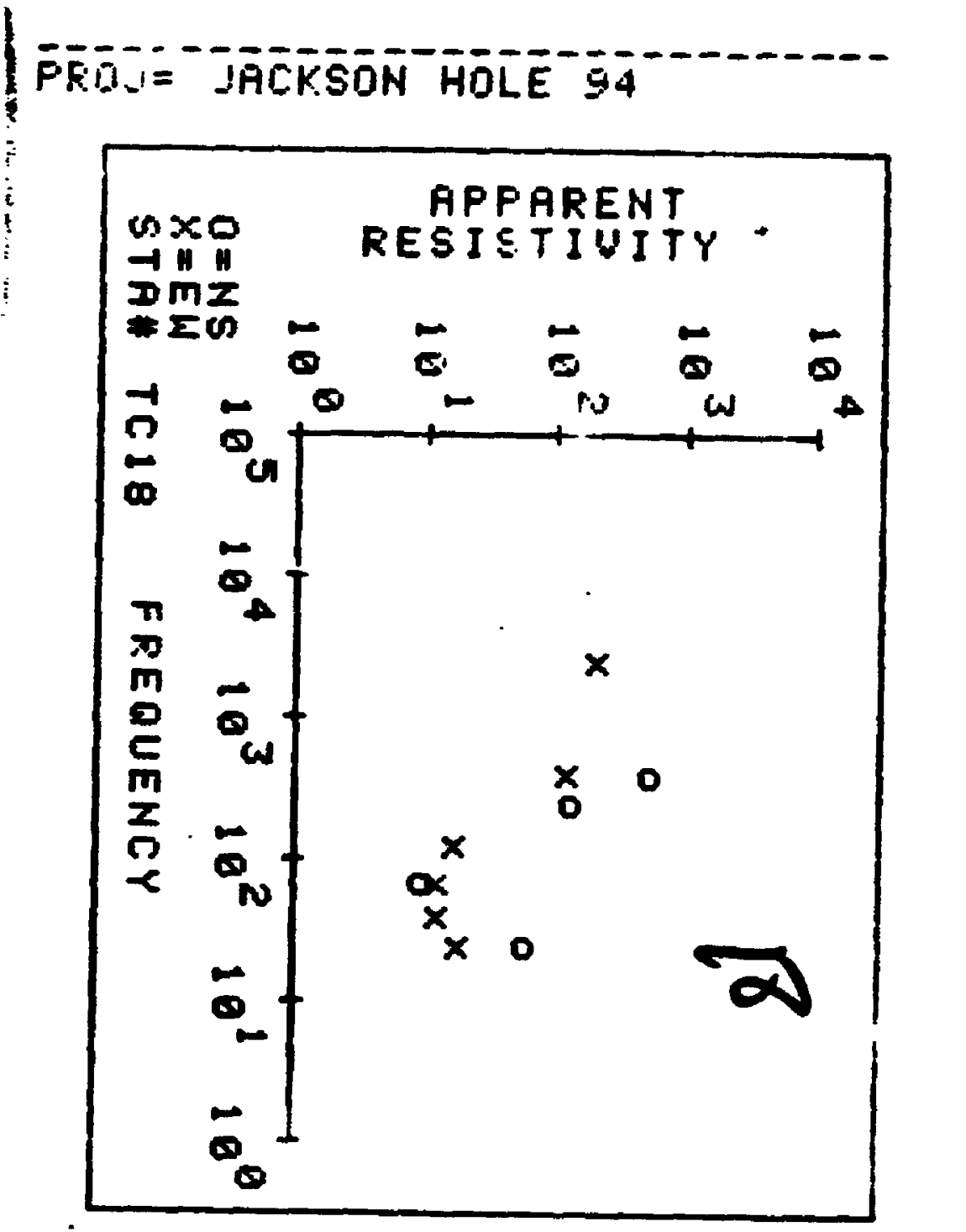
PRODJECTEJACKKSON HOLE

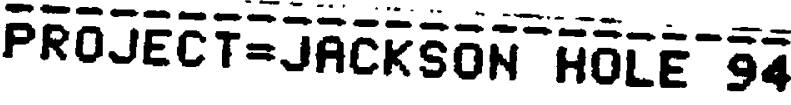

STA. ID_TC19 NS NO FREQ $=5$

$\begin{array}{rrrr}\text { FREQ } & \text { AP-RES } & \text { N OBS } & \text { STD ERR } \\ 14.8 & 228.09 & 3 & 15.97 \\ 27.6 & 259.43 & 4 & 13.36 \\ 148.6 & 1251.59 & 3 & 617.35 \\ 459.0 & 924.57 & 5 & 264.89 \\ 7500.8 & 582.09 & 3 & 17.69\end{array}$

STA. ID_TC19 EW NO FREQ $=10$

\begin{tabular}{rrrr}
\multicolumn{1}{r}{ FREQ } & AP-RES & N OBS & STD ERR \\
4.5 & 6.68 & 3 & .98 \\
14.6 & 2.24 & 4 & .16 \\
27.6 & 3.63 & 5 & .16 \\
75.6 & 7.38 & 3 & .16 \\
140.0 & 11.77 & 4 & .53 \\
278.6 & 111.71 & 4 & 8.28 \\
450.0 & 31.64 & 4 & 2.68 \\
756.0 & 5.87 & 3 & 1.76 \\
4506.0 & 43.63 & 4 & 4.21 \\
506.0 & 39.75 & 4 & 2.04
\end{tabular}

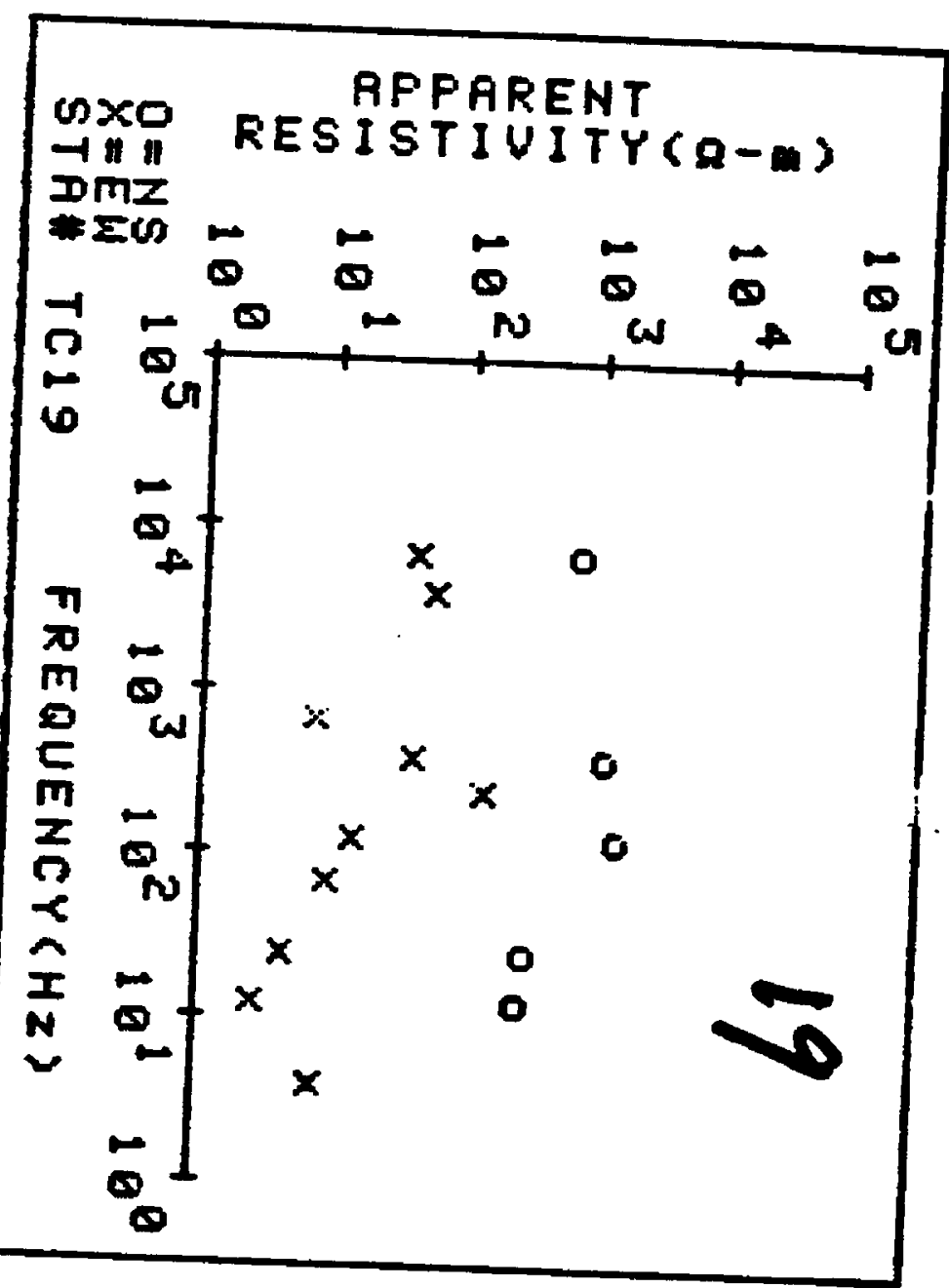

STA. IO-TC2QA HS NO FRER 14 UE

(4)

$\because$ FREQ AP-RES N OBS STO ERR

$i \quad 4.52262 .20 \cdots 11 \quad 1012.70$

$\mathbf{7 . 5} 1422.10 \ldots 5$ 5 337.85

$27.8419 .04-14=238.18$

$45.01473 .60 \div 17 \ldots 340.33$

$\because 75.0811 .51 \quad 22 \quad 102.07$

$140.0=748.79 \quad 21=\ldots 83.44$

$\because 278.9782 .25: 23 \% \quad 58.40$

$458.0622 .49 \div 23 \therefore 57.54$

$758.01831 .80 \cdots 18 \ldots 73.35$

$1400.01443 .08 \quad 23-62.96$

$2700.0719 .67 \because 17 \quad 96.84$

$4508.0 \quad 698.57 \quad 221 \cdots 47.17$

7509.9374 .1123346 .62

27800.65 .65 . $21 \quad 6.65$

8TA. IOD_TC2OA EH NO FREQ 14 14

FREQ AP-RES $N$.OBS STO ERR

4.59836 .80 M 5961.90

$14.08364 .20 \quad 13.1999 .20$

$27.04077 .80 \quad 21 \quad 1279.68$

$75.02874 .80 \quad 23 \quad 305.96$

$148.01782 .09 \quad 24 \quad 208.48$

$278.6 \quad 833.61 \quad 23,49.61$.

$\begin{array}{llll}450.8 & 923.64 & 23 & 46.24\end{array}$

$750.01674 .50 \quad 21 \div 323.84$

1400.0 2109.50 $21 \quad 279.09$

$\begin{array}{llll}2700.0 & 710.09 & 22 & 38.73\end{array}$

$\begin{array}{llll}4500.0 & 575.74 & 21 & 76.17\end{array}$

$7500.0288 .52 \quad 21 \quad 46.10$

$14808.8 \quad 421.86 \quad 13 \quad 48.99$

$27000.0 \cdot 67.08$ 11 13.17
APPARENT

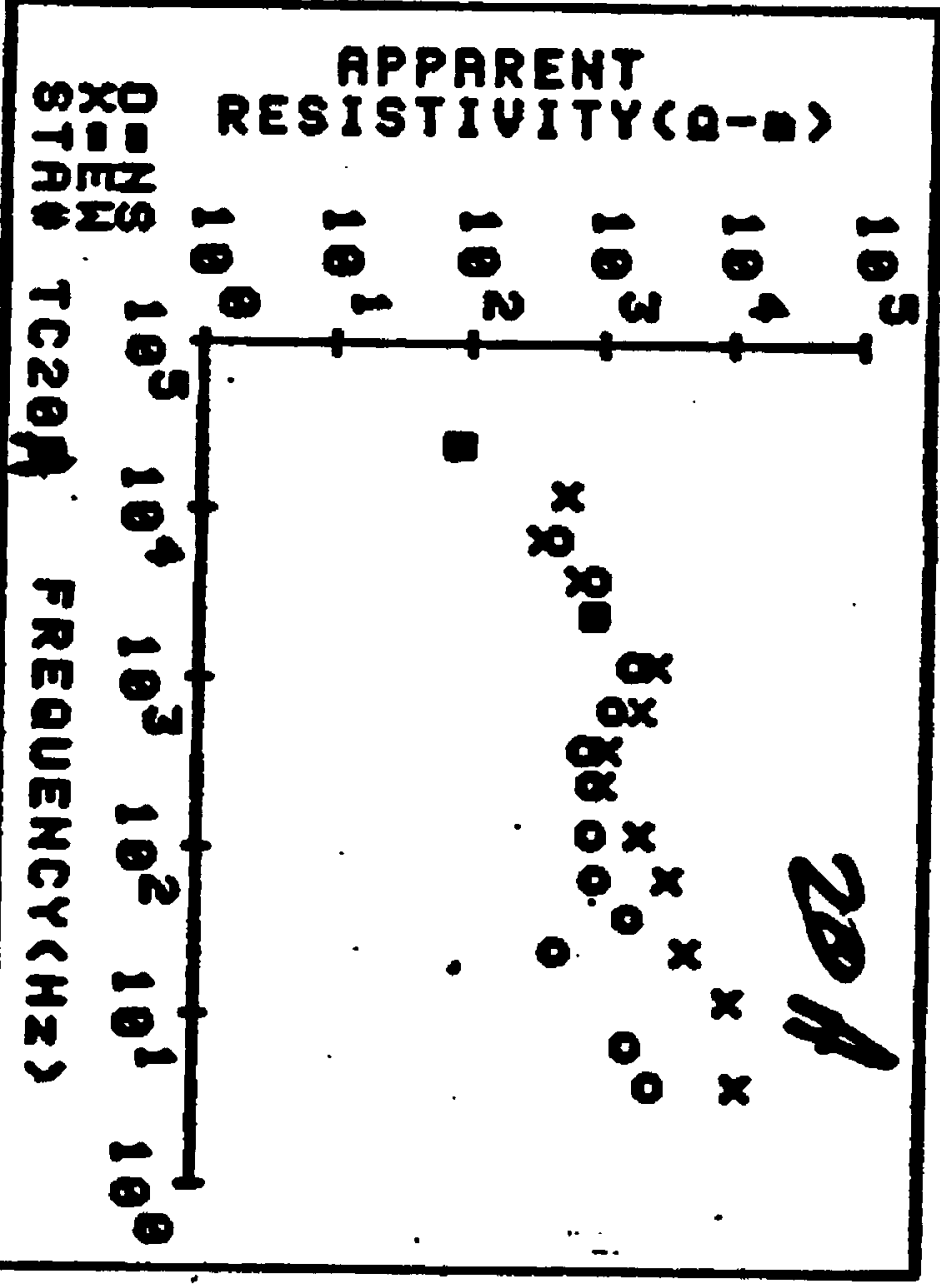


STA. ID_TCZIA NS MO FREQ $=13$

\begin{tabular}{|c|c|c|}
\hline $\begin{array}{r}\text { FREQ } \\
4.5 \\
14.0 \\
27.8 \\
45.8 \\
75.0 \\
148.8 \\
278.0 \\
450.0 \\
750.0 \\
709.0\end{array}$ & $\begin{array}{r}\text { AP-RES } \\
174.20 \\
221.27 \\
374.78 \\
458.45 \\
453.13 \\
167.48 \\
148.08 \\
159.89 \\
177.72 \\
799.88 \\
202.70 \\
155.26 \\
64.63\end{array}$ & 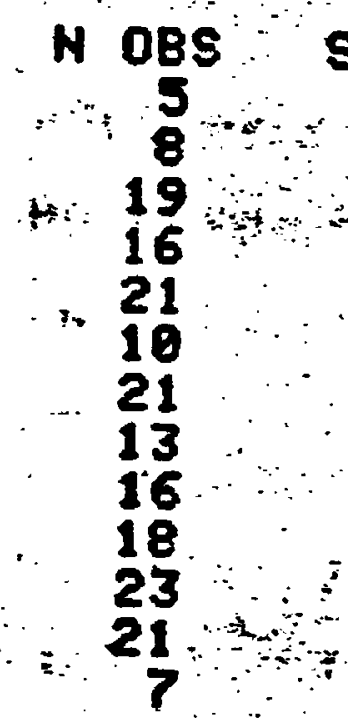 \\
\hline
\end{tabular}

STA. ID_TC21A EH NO FREQ $=13$

$\begin{array}{rrrr}\text { FREQ } & \text { AP-RES } & \text { N OBS } & \text { STD ERR } \\ 4.5 & 258.55 & 10 & 315.44 \\ 14.0 & 84.60 & 18 & 21.72 \\ 27.0 & 198.86 & 20 & 39.30 \\ 45.0 & 445.25 & 14 & 44.52 \\ 75.0 & 240.67 & 21 & 37.10 \\ 140.0 & 291.93 & 19 & 30.62 \\ 270.0 & 177.33 & 23 & 22.98 \\ 450.0 & 133.86 & 11 & 26.21 \\ -750.0 & 235.26 & 10 & 25.54 \\ 2700.0 & 327.03 & 21 & 39.17 \\ 4509.0 & 156.66 & 19 & 19.94 \\ 7509.0 & 269.46 & 23 & 9.83 \\ 4000.0 & 51.44 & 6 & 25.51\end{array}$

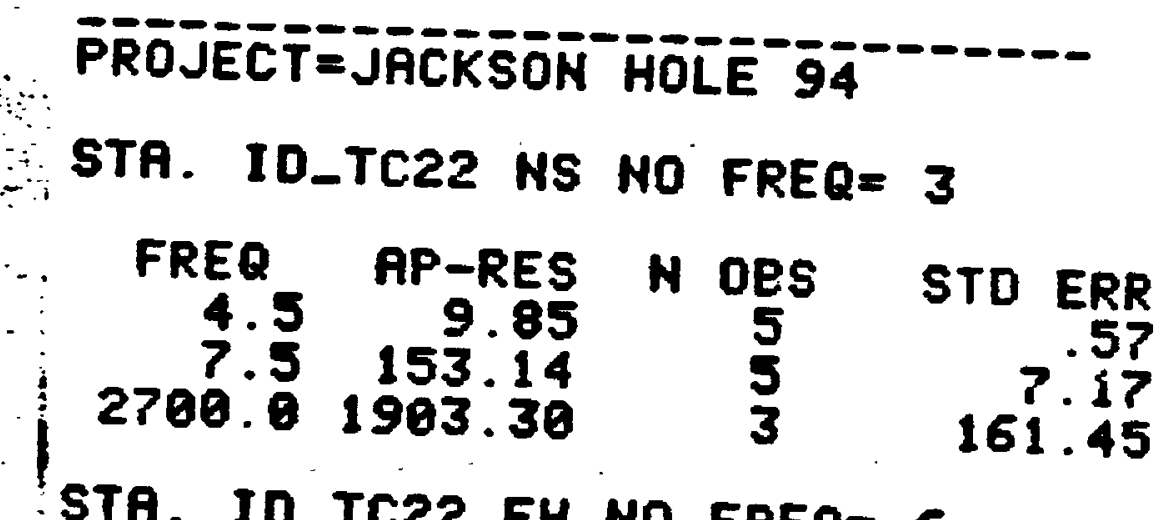

STA. ID_TC22 EH NO FREQ $=6$

FREQ AP-RES N OBS STD ERR

$\begin{array}{rrrr}7.5 & 51.25 & 4 & 1.12\end{array}$

$27.012 .78 \quad 4 \quad .78$

$\begin{array}{llll}45.8 & 28.38 & 3 & 2.85\end{array}$

$\begin{array}{llll}450.8 & 75.15 & 3 & 3.52\end{array}$

$\begin{array}{llll}750.0 & 137.69 & 5 & 9.41\end{array}$
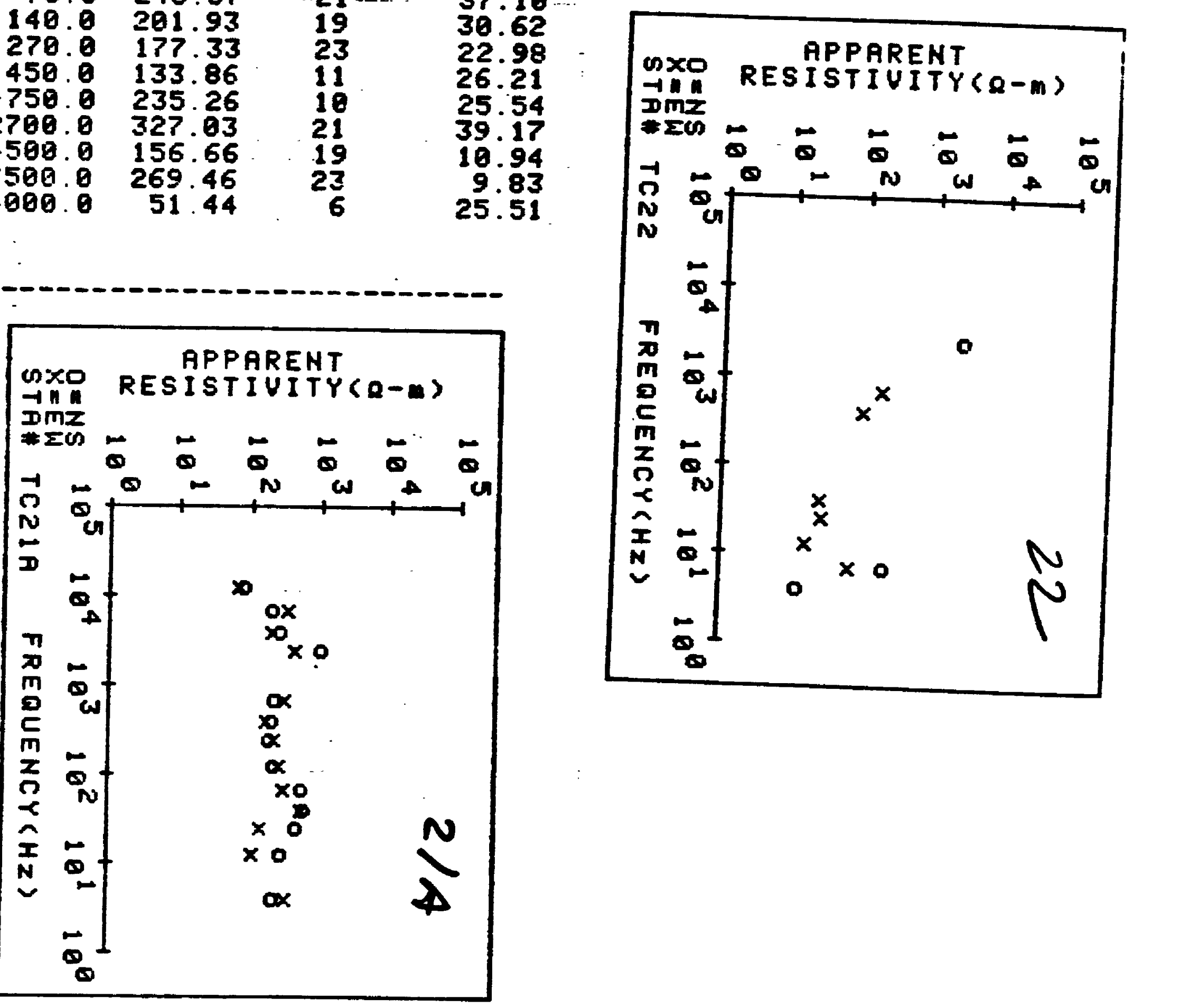
PROAJECT =JACKSON HOLE פ̈.

STA. ID_TC23 NS NO FREQ = ?

FREQ AP-RES N OBS STD ERR

14.0518 .56

$27.0 \quad 894.27$

$45.0 \quad 233.32$

75.0416 .57

$140.0 \quad 233.72$

$450.0 \quad 397.51$

2700.01985 .90

$$
\begin{array}{rr}
\text { OES } & \text { STDERR } \\
6 & 19.07 \\
4 & 111.65 \\
6 & 22.32 \\
4 & 1.63 \\
5 & 5.40 \\
3 & 6.02 \\
\hline & 178.73
\end{array}
$$

STA. ID_TC23 EN HO FREQ = ?

\begin{tabular}{rccr}
\multicolumn{1}{r}{ FREQ } & AP-RES & N OBS & STD ERR \\
7.5 & 359.62 & 1 & 0.00 \\
27.0 & 209.61 & 3 & 5.26 \\
45.0 & 396.53 & 4 & 29.48 \\
75.0 & 971.97 & 4 & 16.61 \\
140.0 & 629.46 & 6 & 23.91 \\
450.0 & 849.89 & 7 & 37.16 \\
2780.0 & 34.950 & 4 & 94.15
\end{tabular}

STA. ID_TC24B NS NO FREQ $=15$

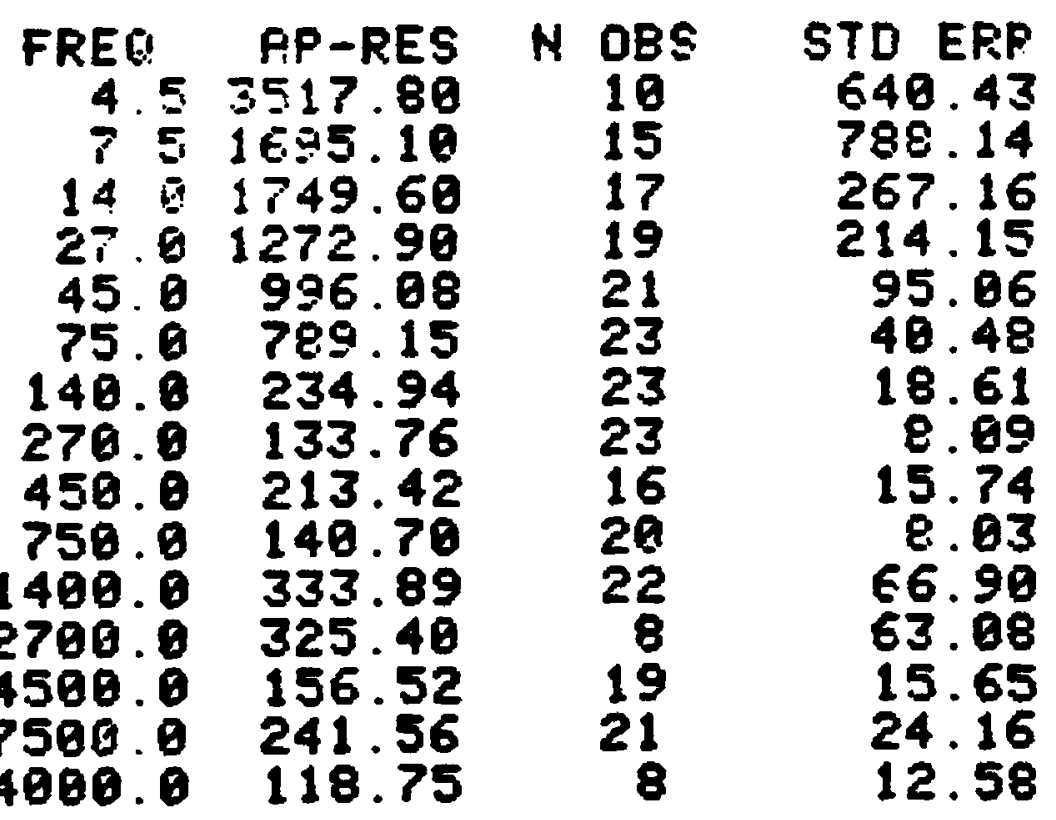

STA. ID_TC24E EH NO FREQ $=15$

FREQ AP-RES $H$ OBS STD ERR $4.51884 .48 \quad 6 \quad 334.18$

$7.5507 .03 \quad 9 \quad 104.79$

$\begin{array}{llll}14.0 & 544.58 & 13 & 226.82\end{array}$

$27.8 \quad 865.10 \quad 21 \quad 231.29$

$\begin{array}{llll}45.0 & 542.57 & 23 & 53.25\end{array}$

$75.0 \quad 456.98 \quad 21 \quad 41.31$

$140.9 \quad 130.14 \quad 24 \quad 12.11$

$276.0 \quad 150.36 \quad 22 \quad 26.26$

$450.0 \quad 198.42 \quad 21 \quad 9.00$

$750.0 \quad 437.67 \quad 21 \quad 22.41$

$\begin{array}{llll}1400.0 & 719.66 & 23 & 101.08\end{array}$

$2700.01348 .70 \quad 7 \quad 489.78$

$\begin{array}{llll}4500.0 & 966.85 & 23 & 109.03\end{array}$

$\begin{array}{llll}7500.8 & 473.69 & 23 & 74.19\end{array}$

$14000.0 \quad 559.11 \quad 14 \quad 77.04$

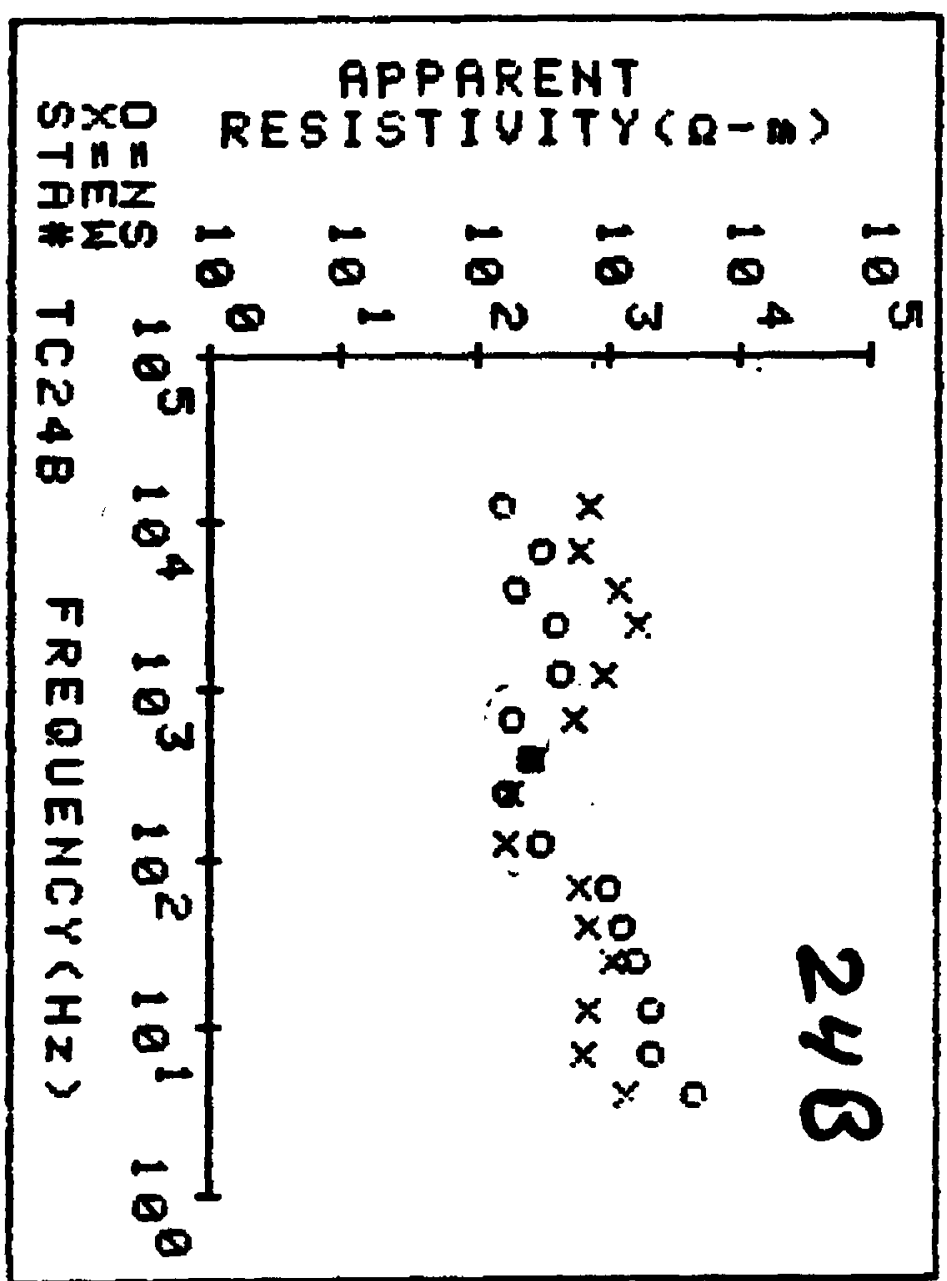


STA. ID_TCZSH NS NÜ FREQ $=16$

FREQ AP-FES $N$ ORS STO ERR

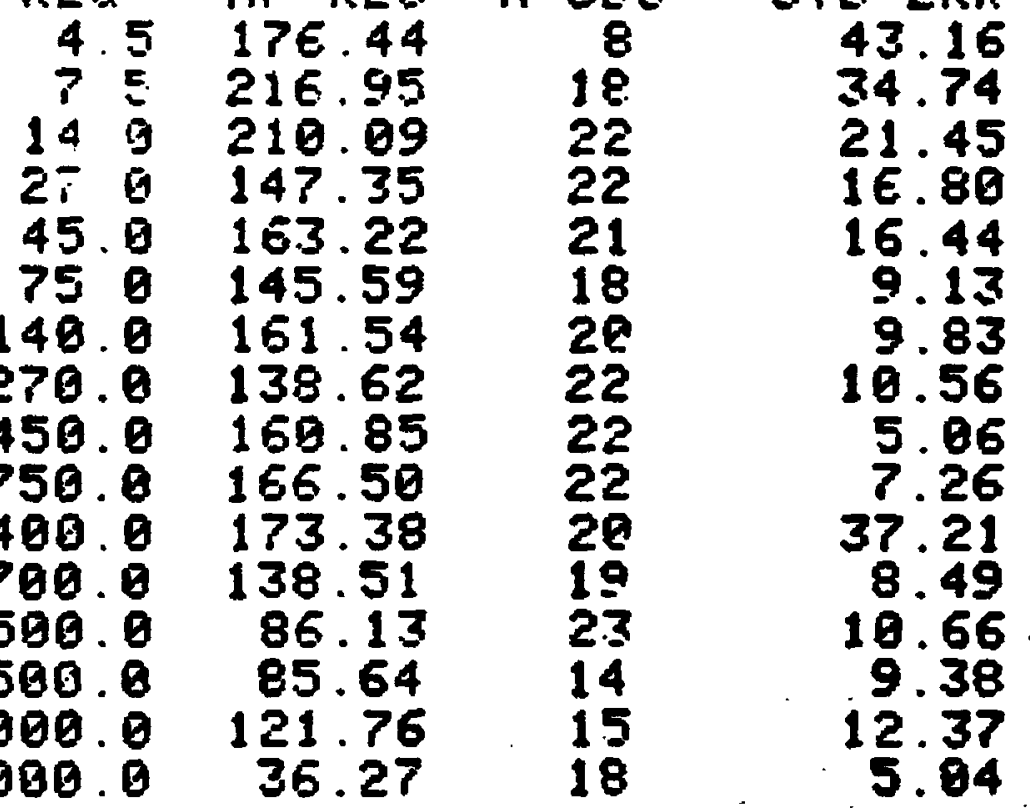

STA. ID_TC2 IA EW NO FREQ $=13$

FREO AP-RES $N$ OES STD ERR $4.5 \quad 557.28$

$7.5 \quad 27273$

$14.0 \quad 372.95$

$27.0 \quad 396.55$

$45.9 \quad 578.42$

$75.0 \quad 339.18$

$140.0 \quad 275.10$

279.9392 .49

450.0276 .85

750.0457 .99

$2700.0 \quad 194.15$

4500.0113 .99

7500.0
8

11

17

15

16

11

17

15

12

21

23

20
108.99

79.99

37.78

55.04

27.49

39.17

24.61

109.51

5.63

16.22

9.99
111.97

\section{PROTJECT = JACKSON HOLE}

STA. ID_TC26 NS NO FREQ $=10$

FREQ AP-RES N OBS STD ERR $\begin{array}{rrrr}4.5 & 13.28 & 2 & .34 \\ 7.5 & 7.64 & 4 & .36\end{array}$

$14.828 .54 \quad 7 \quad 2.68$

$\begin{array}{llll}27.0 & 14.29 & 8 & 1.42\end{array}$

$\begin{array}{llll}45.0 & 23.96 & 8 & 3.24\end{array}$

$140.0 \quad 26.63$

$270.0 \quad 23.71$

$450.0 \quad 35.96$

$750.0 \quad 15.68$

7580.0

9.45

$8 \quad 8.27$

$10 \quad 3.58$

$? \quad 1.67$

STA. ID_TC26 EW HO FREQ $=11$

FREQ AP-RES $N$ OBS STO ERR

$4.57 .04 \quad-5 \ldots . .43$

$7.5 \quad 3.83 \quad 7 \quad .59$

14.9

27.0

9.71

1.39

75.6

148.0

13.86

11.38

9.73

9.68

270.8

13.69

$458.0 \quad 17.74$

750.014 .85

7500.0

4.22

27800.0

3.84

.27

1.89

1.18

2.27

1.50

1.04

.19

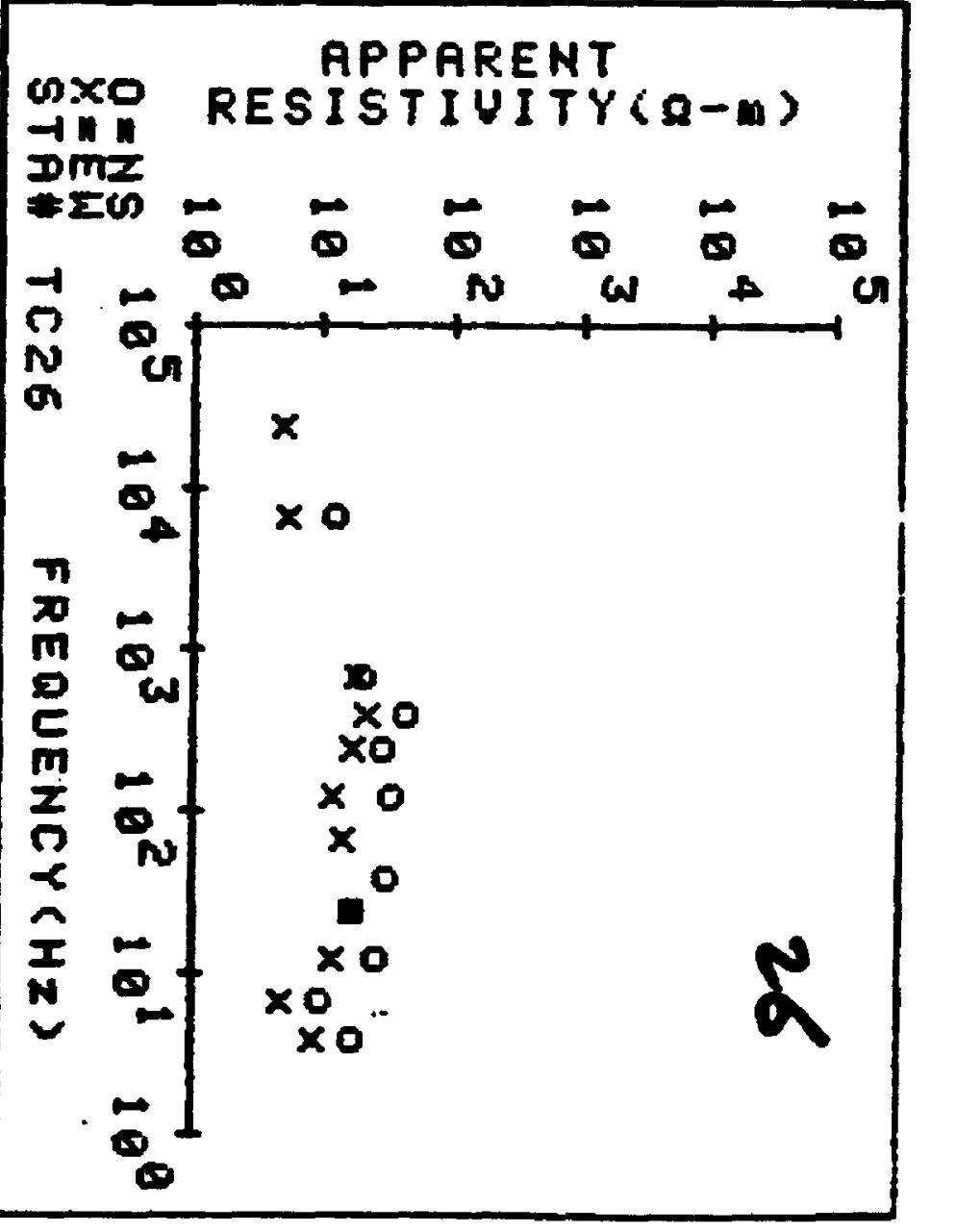


PROJJECT =JACKSON HOLE 94

STA. ID_TC27 NS NO FREQ $=14$

FREQ AF-FEES N OBS STD ERR

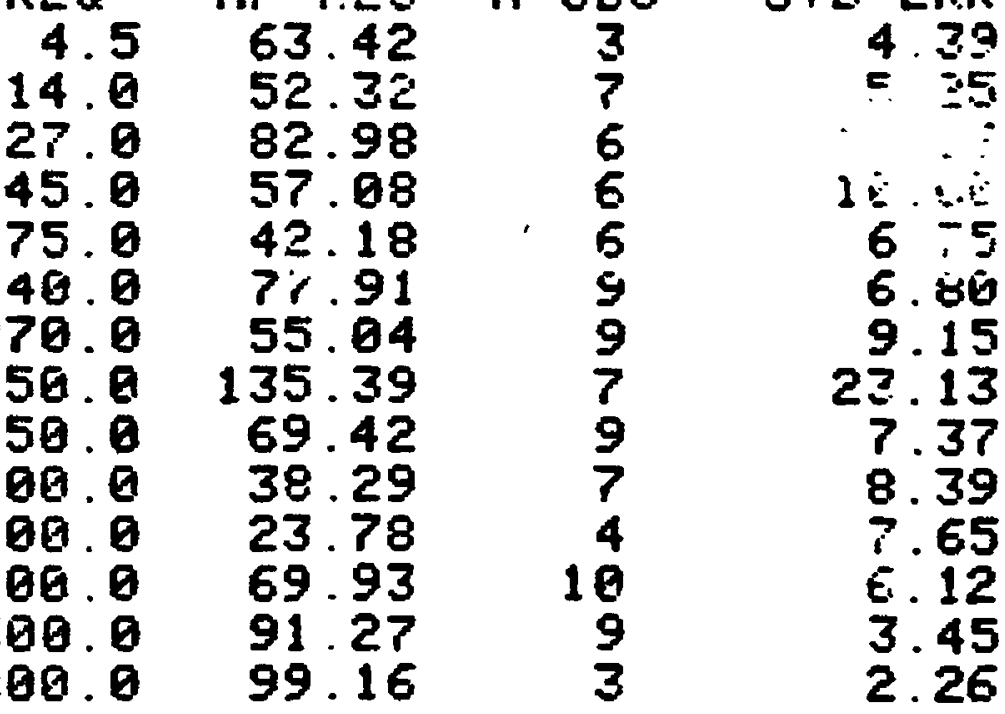

STA. ID_TC27 EW HO FREQ $=15$

\begin{tabular}{|c|c|c|c|c|}
\hline $\begin{array}{r}\text { FREQ } \\
4.5 \\
14.5 \\
24.0 \\
45.0 \\
75.0 \\
140.0 \\
270.9 \\
450.0 \\
750.0 \\
490.0 \\
230.0 \\
560.0\end{array}$ & $\begin{array}{r}\text { AP-RES } \\
67.67 \\
34.79 \\
79.23 \\
89.54 \\
65.28 \\
87.27 \\
58.25 \\
42.15 \\
49.77 \\
22.53 \\
12.78 \\
26.73 \\
31 \cdot 34 \\
83.55 \\
75 \cdot 73\end{array}$ & & $\begin{array}{c}085 \\
3 \\
8 \\
5 \\
7 \\
9 \\
9 \\
8 \\
8 \\
6 \\
7 \\
5 \\
3 \\
6 \\
6 \\
3\end{array}$ & $\begin{array}{r}\text { STD ERR } \\
.72 \\
6.92 \\
29.73 \\
12.18 \\
5.70 \\
7.99 \\
5.66 \\
3.31 \\
4.10 \\
1.42 \\
2.01 \\
1.18 \\
4.03 \\
=.19\end{array}$ \\
\hline
\end{tabular}

PROJECT $=$ JACKSON HOLE 94

STA. ID_TC28 NS NO FREQ $=12$

FREQ AP-RES N OBS STD ERR

$\begin{array}{rrrr}7.5 & 50.82 & 6 & 5.14 \\ 14.0 & 20.36 & 8 & 1.70 \\ 27.0 & 25.59 & 9 & 2.95 \\ 45.0 & 48.48 & 9 & 8.53 \\ 75.0 & 80.95 & 7 & 16.58 \\ 40.0 & 98.74 & 19 & 7.24 \\ 70.0 & 150.30 & 6 & 11.91 \\ 50.0 & 204.19 & 8 & 31.63 \\ 500.0 & 150.56 & 5 & 15.69 \\ 900.0 & 153.72 & 19 & 12.07 \\ 900.0 & 128.29 & 3 & 37.30 \\ 900.0 & 81.48 & 3 & 31.55\end{array}$

$27000.0 \quad 81.48$

STA. ID_TC28 EN NO FREQ $=15$

FREQ AP-RES N OBS STD ERR

$\begin{array}{rrrr}4.5 & 20.21 & 2 & 2.79 \\ 7.5 & 17.84 & 8 & 1.66 \\ 14.0 & 21.93 & 7 & 2.36 \\ 27.0 & 29.34 & 10 & 2.71 \\ 45.0 & 31.99 & 8 & 4.85 \\ 75.0 & 40.58 & 8 & .85 \\ 40.0 & 58.89 & 10 & 4.51 \\ 70.0 & 54.52 & 8 & 1.32 \\ 50.0 & 35.99 & 4 & 11.85 \\ 50.0 & 72.30 & 19 & 6.91 \\ 90.9 & 81.19 & 3 & 1.01 \\ 00.0 & 49.73 & 3 & .77 \\ 900.0 & 95.07 & 10 & 7.05 \\ 900.0 & 238.59 & 2 & 9.37\end{array}$

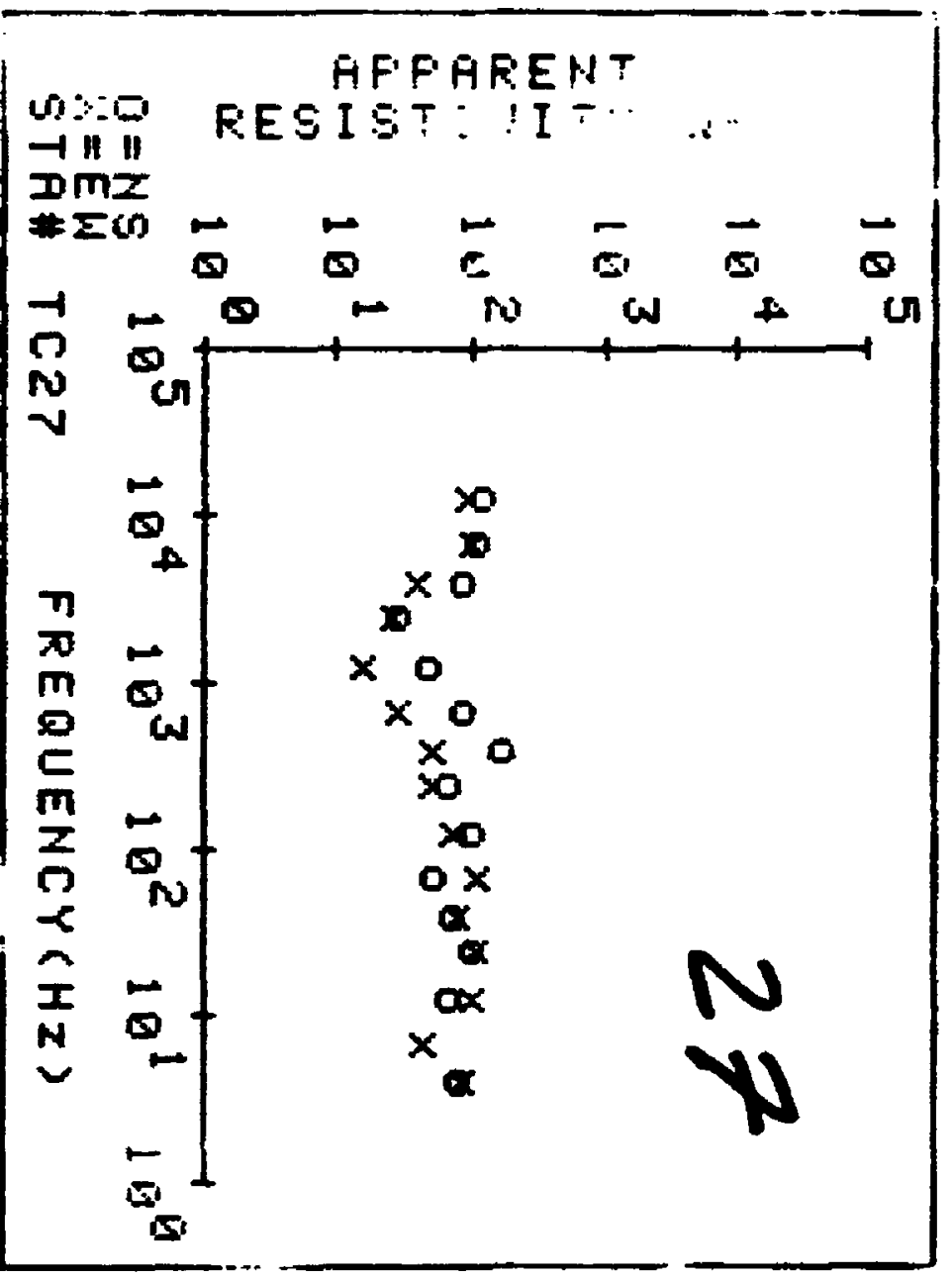

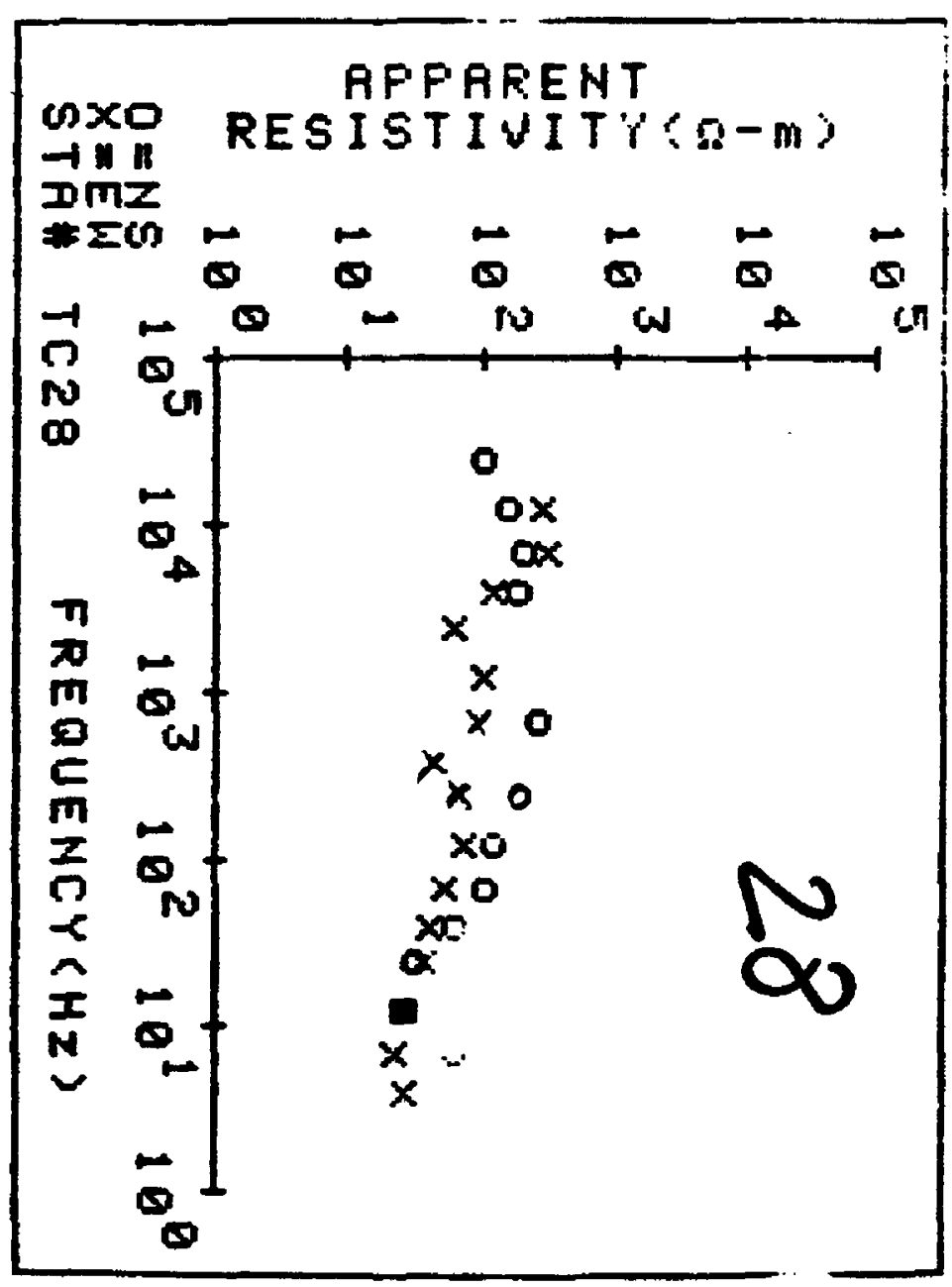


STA. ID_TC29 NS NO FREQ $=15$

\begin{tabular}{|c|c|c|c|}
\hline $\begin{array}{r}\text { FREQ } \\
4.5 \\
7.5 \\
14.0 \\
27.0 \\
45.0 \\
75.0 \\
140.0 \\
270.0 \\
450.0 \\
750.0 \\
490.0 \\
760.0\end{array}$ & $\begin{array}{r}\text { AP-RES } \\
12.93 \\
18.11 \\
13.17 \\
11.55 \\
22.22 \\
32.83 \\
33.88 \\
39.12 \\
59.83 \\
76.43 \\
108.92 \\
53.16 \\
75.08 \\
199.95 \\
47.08\end{array}$ & $\begin{array}{c}\text { N OBS } \\
3 \\
4 \\
6 \\
6 \\
6 \\
9 \\
9 \\
9 \\
19 \\
19 \\
9 \\
8 \\
2 \\
3 \\
3 \\
4 \\
7 \\
3\end{array}$ & \\
\hline
\end{tabular}

STA. ID_TC29 EW NO FREQ $=13$

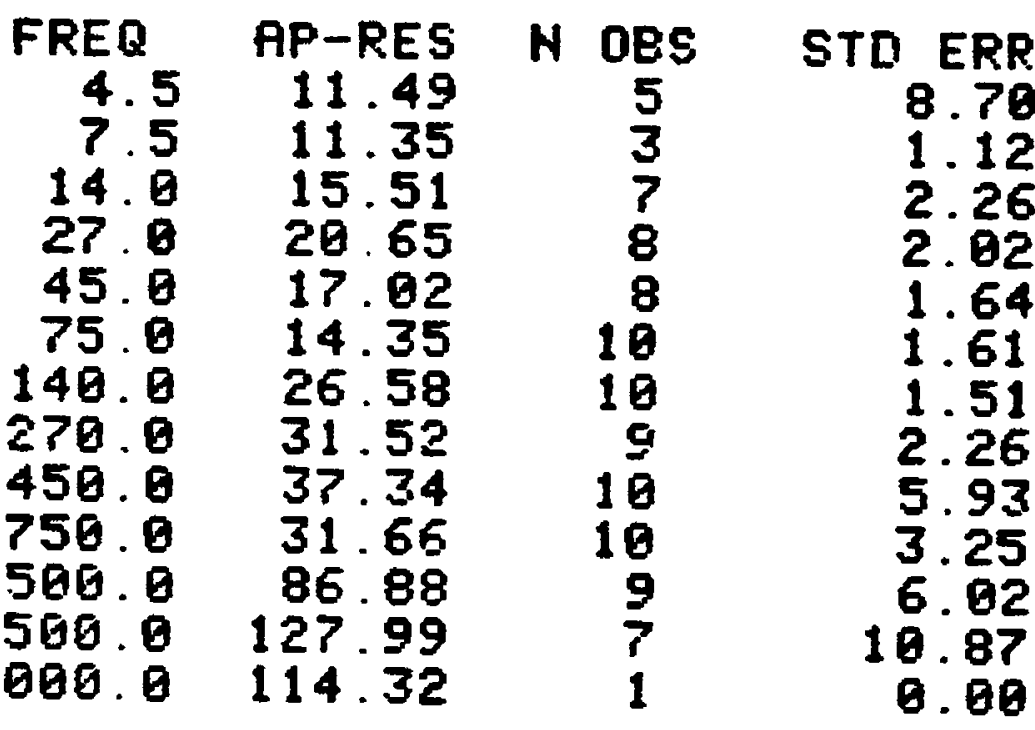

PRŌJEC $\bar{T}=\bar{T}=\bar{J} \bar{C} \bar{S} \bar{S} \bar{O}-\overline{H O L E} \overline{9}$

STA. ID_TC3日 NS NO FREQ $=11$

FREQ AP-RES $N$ OES STD ERR $\begin{array}{llll}4.5 & 20.01 & 2 & 9.78\end{array}$

$\begin{array}{llll}14.0 & 58.38 & 6 & 7.07\end{array}$

$\begin{array}{llll}27.6 & 61.97 & 7 & 5.21\end{array}$

$75.0 \quad 157.25 \quad 5 \quad 37.85$

$140.0 \quad 103.07 \quad 8 \quad 13.91$

$\begin{array}{llll}270.0 & 48.26 & 3 & 4.46\end{array}$

$\begin{array}{llll}450.0 & 95.81 & 8 & 26.35\end{array}$

$\begin{array}{lllll}750.0 & 129.17 & 8 & 15.22\end{array}$

$\begin{array}{llll}4500.0 & 103.78 & 6 & 13.60\end{array}$

$\begin{array}{llll}7500.0 & 188.76 & 8 & 31.12\end{array}$

$\begin{array}{llll}14000.0 & 173.03 & 3 & 35.06\end{array}$

STA. ID_TC30 EK NO FREQ $=14$

FREQ AP-RES N OBS STD ERR

$\begin{array}{llll}4.5 & 6.19 & 2 & .46\end{array}$

$\begin{array}{llll}14.0 & 44.79 & 8 & 5.72\end{array}$

$\begin{array}{lllll}27 & 0 & 72.84 & 8 & 29.91\end{array}$

$\begin{array}{llll}45.0 & 123.36 & 7 & 17.17\end{array}$

$\begin{array}{llll}75.6 & 175.43 & 7 & 36.32\end{array}$

$\begin{array}{llll}140.0 & 77.62 & 8 & 8.04\end{array}$

$\begin{array}{llll}270.0 & 117.00 & 8 & 13.26\end{array}$

$450.0 \quad 172.79 \quad 5 \quad 13.66$

$\begin{array}{llll}750.0 & 191.49 & 8 & 26.63\end{array}$

$\begin{array}{llll}1400.0 & 472.47 & 1 & 0.00\end{array}$

$4500.0 \quad 187.10 \quad 6 \quad 21.50$

$7500.0 \quad 377.06 \quad 4 \quad 9.67$

$14090 \quad 360.00 \quad 4 \quad 20.41$

27006 o $76.28 \quad 1 \quad 0.01$

FFU JACKSEN HU. E
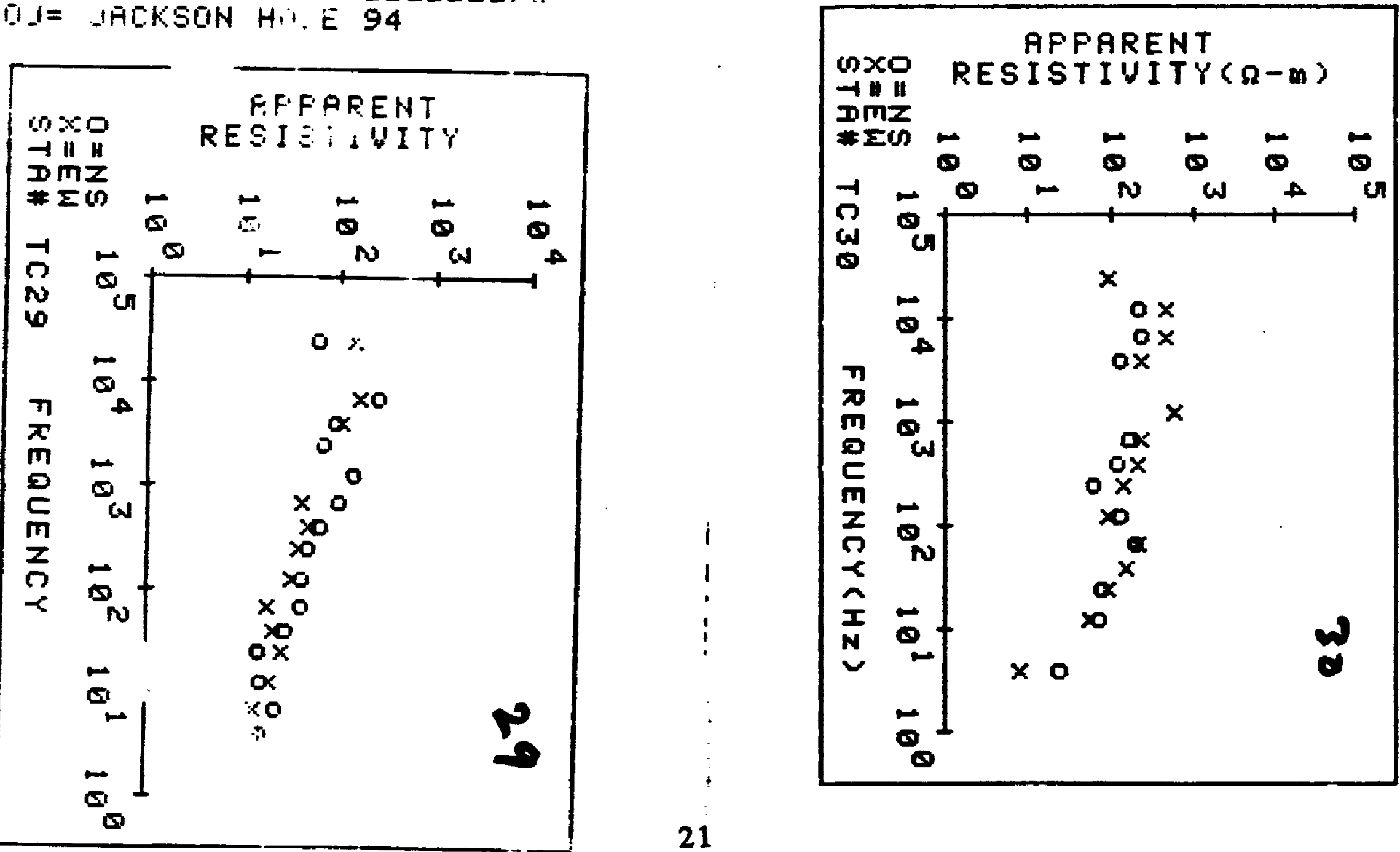


$\begin{array}{rccr}\text { STA. ID_TC31 } & \text { NS NO FREQ= } \\ \text { FREQ } & \text { AP-RES } & \text { N OBS } & \text { STO ERR } \\ 4.5 & 158.43 & 1 & 0.00 \\ 7.5 & 133.23 & 3 & 5.88 \\ 14.0 & 241.55 & 3 & 12.64 \\ 27.0 & 413.76 & 7 & 56.67 \\ 45.0 & 174.62 & 5 & 61.18 \\ 75.0 & 364.94 & 7 & 73.89 \\ 140.0 & 133.79 & 7 & 17.12 \\ 270.0 & 213.38 & 9 & 13.87 \\ 450.0 & 251.28 & 8 & 17.58 \\ 750.0 & 155.79 & 6 & 13.66 \\ 4500.0 & 560.23 & 8 & 29.26 \\ 14600.0 & 295.72 & 3 & 15.92\end{array}$

STA. ID_TC31 EH NO FREQ $=13$

FREQ AP-RES $N$ OBS STD ERR $4.5 \quad 32.18$

14.049 .65

27.0143 .39

$45.0 \quad 372.09$

$75.0 \quad 333.41$

$140.0 \quad 190.99$

$270.0 \quad 173.79$

450.0242 .72

$750.9 \quad 171.30$

$2700.0 \quad 847.83$

$4500.0 \quad 475.49$

7500.0193 .51

14000.0125466

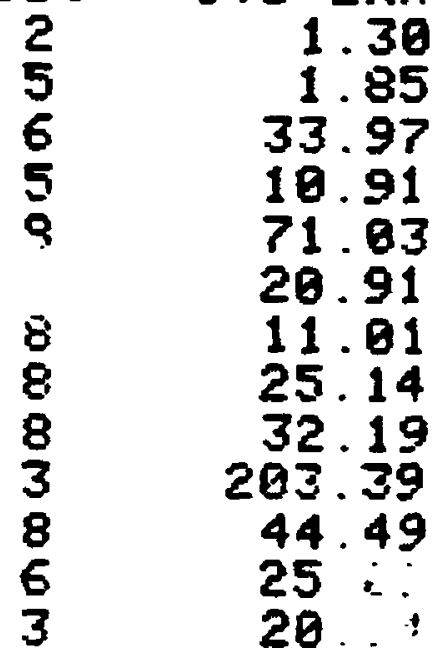

STA. ID_TC32 NS NO FREQ $=14$

\begin{tabular}{|c|c|c|c|}
\hline $\begin{array}{r}\text { FREQ } \\
4.5 \\
7.5 \\
14.0 \\
27.0 \\
45.0 \\
75.0 \\
140.0 \\
270.8 \\
450.0 \\
750.0 \\
4580.0 \\
7500.0\end{array}$ & $\begin{array}{r}\text { AP-RES } \\
423.62 \\
199.20 \\
189.73 \\
289.60 \\
119.66 \\
215.11 \\
229.30 \\
86.69 \\
146.10 \\
177.79 \\
335.54 \\
173.47 \\
66.77 \\
58.45\end{array}$ & $\begin{array}{c}\text { OBS } \\
5 \\
5 \\
8 \\
5 \\
4 \\
3 \\
3 \\
4 \\
8 \\
4 \\
3 \\
7 \\
7 \\
3 \\
1\end{array}$ & $\begin{array}{r}\text { STD ERR } \\
36.47 \\
14.79 \\
7.45 \\
42.78 \\
3.28 \\
14.51 \\
17.87 \\
5.56 \\
7.67 \\
13.34 \\
12.99 \\
6.67 \\
.62 \\
0.00\end{array}$ \\
\hline
\end{tabular}

STA. ID_TC32 EH NO FREQ $=14$

\begin{tabular}{|c|c|c|c|}
\hline $\begin{array}{r}\text { FREQ } \\
4.5 \\
7.5 \\
14.0 \\
27.0 \\
45.0 \\
75.0 \\
140.0 \\
270.0 \\
450.0 \\
750.0 \\
4560.0 \\
7506.0 \\
4060.0\end{array}$ & $\begin{array}{l}\text { AP-RES } \\
162.27 \\
473.58 \\
473.94 \\
328.28 \\
241.76 \\
359.94 \\
223.36 \\
264.67 \\
291.59 \\
359.51 \\
593.82 \\
129.97 \\
285.46\end{array}$ & $\begin{array}{c}\text { H Oes } \\
4 \\
3 \\
4 \\
8 \\
4 \\
5 \\
9 \\
3 \\
7 \\
3 \\
6 \\
3 \\
3\end{array}$ & $\begin{array}{r}\text { STD ERR } \\
31.97 \\
21.53 \\
186.56 \\
66.17 \\
1.20 \\
8.85 \\
21.97 \\
18.94 \\
9.57 \\
4.98 \\
52.91 \\
1.98 \\
16.89 \\
0.90\end{array}$ \\
\hline
\end{tabular}

RU JALEST̃N HOLE ज4

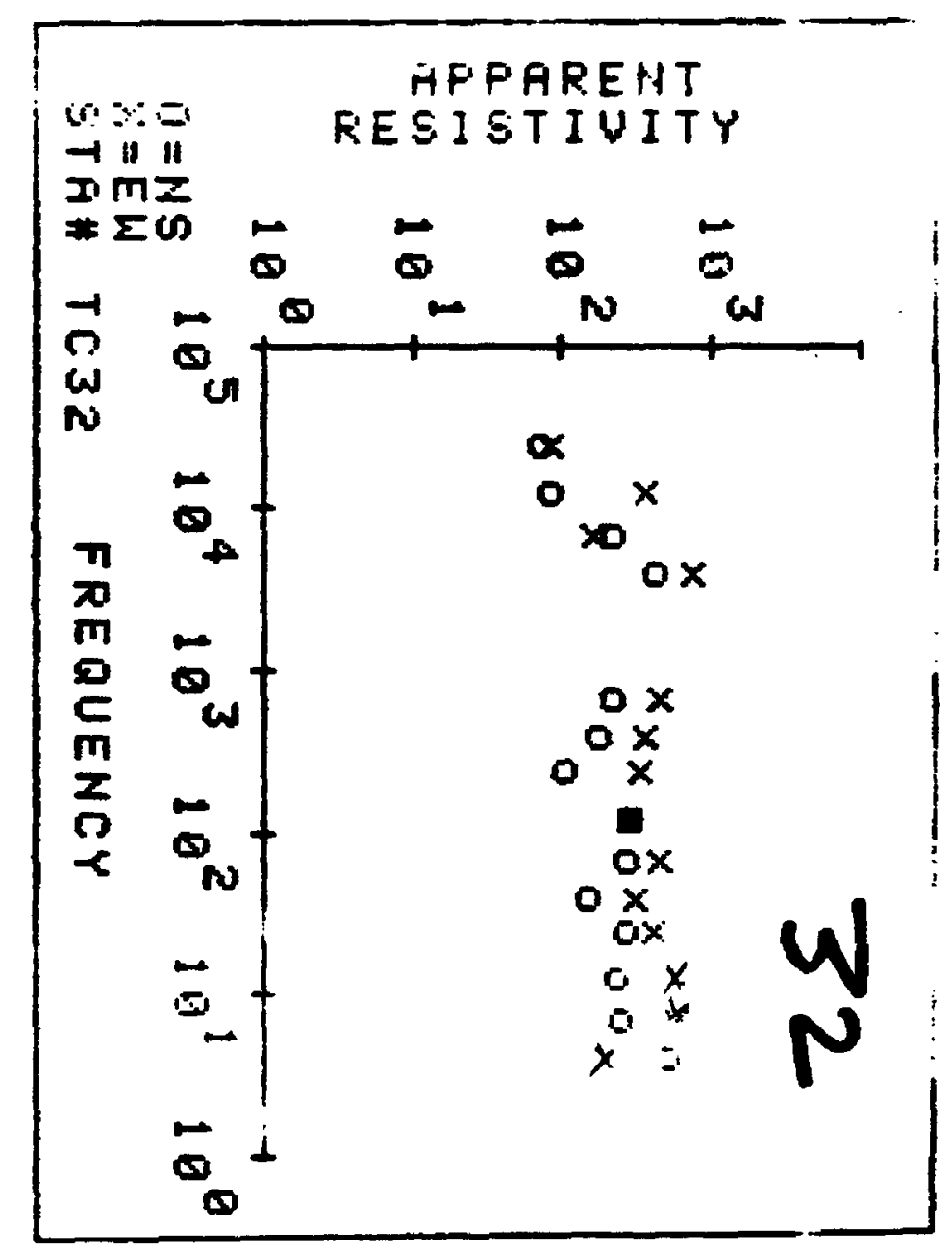


F̄EOJECTT=JĀK

STA. IO BB NS NI FREQ $=12$

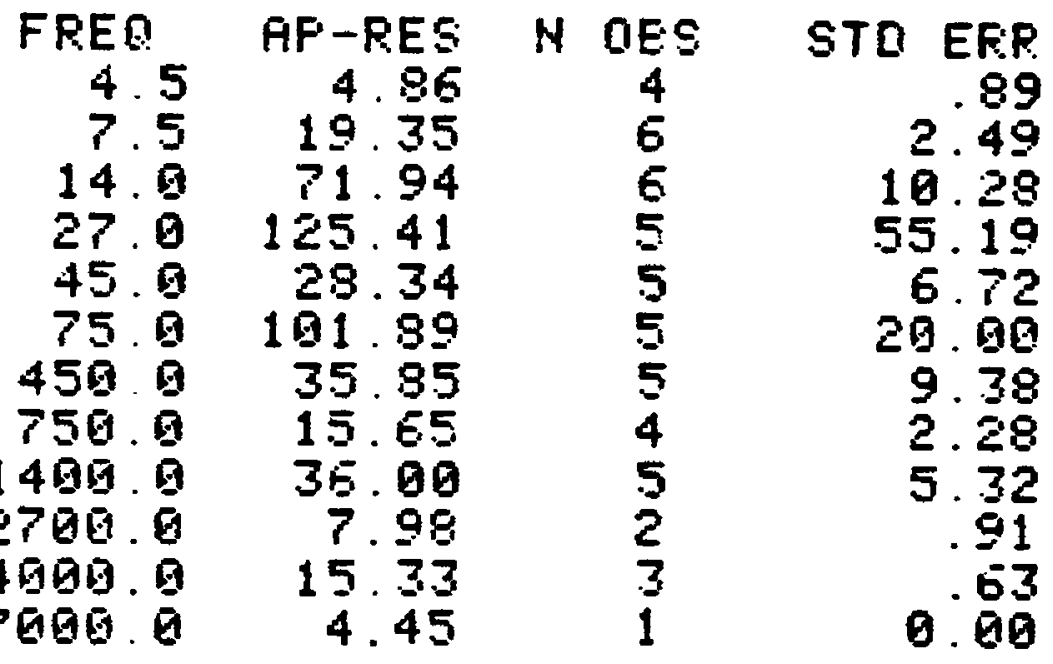

STA. ILIBB EW NO FREQ= 10

\begin{tabular}{|c|c|c|c|}
\hline $\begin{array}{r}\text { FREQ } \\
4.5 \\
7.5 \\
14.9 \\
27.9 \\
45.9 \\
750.9 \\
760.0 \\
500.0 \\
990.9\end{array}$ & $\begin{array}{r}\text { AF-RES } \\
7.56 \\
50.48 \\
199.31 \\
88.55 \\
59.20 \\
87.45 \\
12.98 \\
11.82 \\
87.88 \\
2.96\end{array}$ & $\begin{array}{c}\text { N OES } \\
6 \\
5 \\
5 \\
6 \\
6 \\
4 \\
2 \\
4 \\
3 \\
1\end{array}$ & $\begin{array}{r}\text { STL ERR } \\
.14 \\
18.49 \\
43.27 \\
7.37 \\
14.66 \\
3.92 \\
4.91 \\
1.13 \\
1.34 \\
0.09\end{array}$ \\
\hline
\end{tabular}

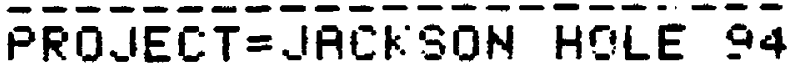

STA. IO_TC34 NS NO FREQ $=14$

\begin{tabular}{|c|c|c|c|}
\hline $\begin{array}{r}\text { FREQ } \\
4.5 \\
7.5 \\
14.0 \\
27.0 \\
45.9 \\
75.9 \\
149.9 \\
270.9 \\
459.9 \\
750.0 \\
509.0 \\
590.0 \\
1909.0 \\
909.9\end{array}$ & $\begin{array}{r}\text { AF-RES } \\
157.45 \\
48.51 \\
64.44 \\
29.23 \\
48.23 \\
122.35 \\
31.91 \\
83.28 \\
118.74 \\
79.92 \\
69.49 \\
41.07 \\
64.69 \\
142.93\end{array}$ & $\begin{array}{c}\text { N DES } \\
3 \\
7 \\
7 \\
8 \\
7 \\
6 \\
8 \\
9 \\
6 \\
4 \\
9 \\
7 \\
7 \\
1\end{array}$ & $\begin{array}{r}\text { STL ERF } \\
14.92 \\
5.54 \\
19.66 \\
3.91 \\
12.60 \\
1.90 \\
8.24 \\
7.53 \\
33.96 \\
2.33 \\
9.81 \\
1.98 \\
3.88 \\
0.09\end{array}$ \\
\hline
\end{tabular}

STA. IO_TC34 EW NO FREQ $=12$

FREQ AF-RES N DES STL ERR

$\begin{array}{llll}4.5 & 148.42 \quad 3 & 7.17\end{array}$

$\begin{array}{lll}7.5 & 234.95 & 3\end{array}$

14.9285 .10 P 22.15

$45.0 \quad 348.20$ E 25.30

$75.9468 .68 \quad 5 \quad 20.38$

$1400 \quad 615.62 \quad 5 \quad 90.90$

$270.0 \quad 289.89 \quad 7 \quad 27.38$

$750.0134 .39 \quad 5 \quad 10.53$

$\begin{array}{llll}2799.0 & 24.84 & 7 & 1.03\end{array}$

$7500.9 \quad 17.41 \quad 3 \quad 1.76$

$14900.9 \quad 28.46 \quad 3 \quad 1.82$

$27000.019 .99 \quad 2.99$
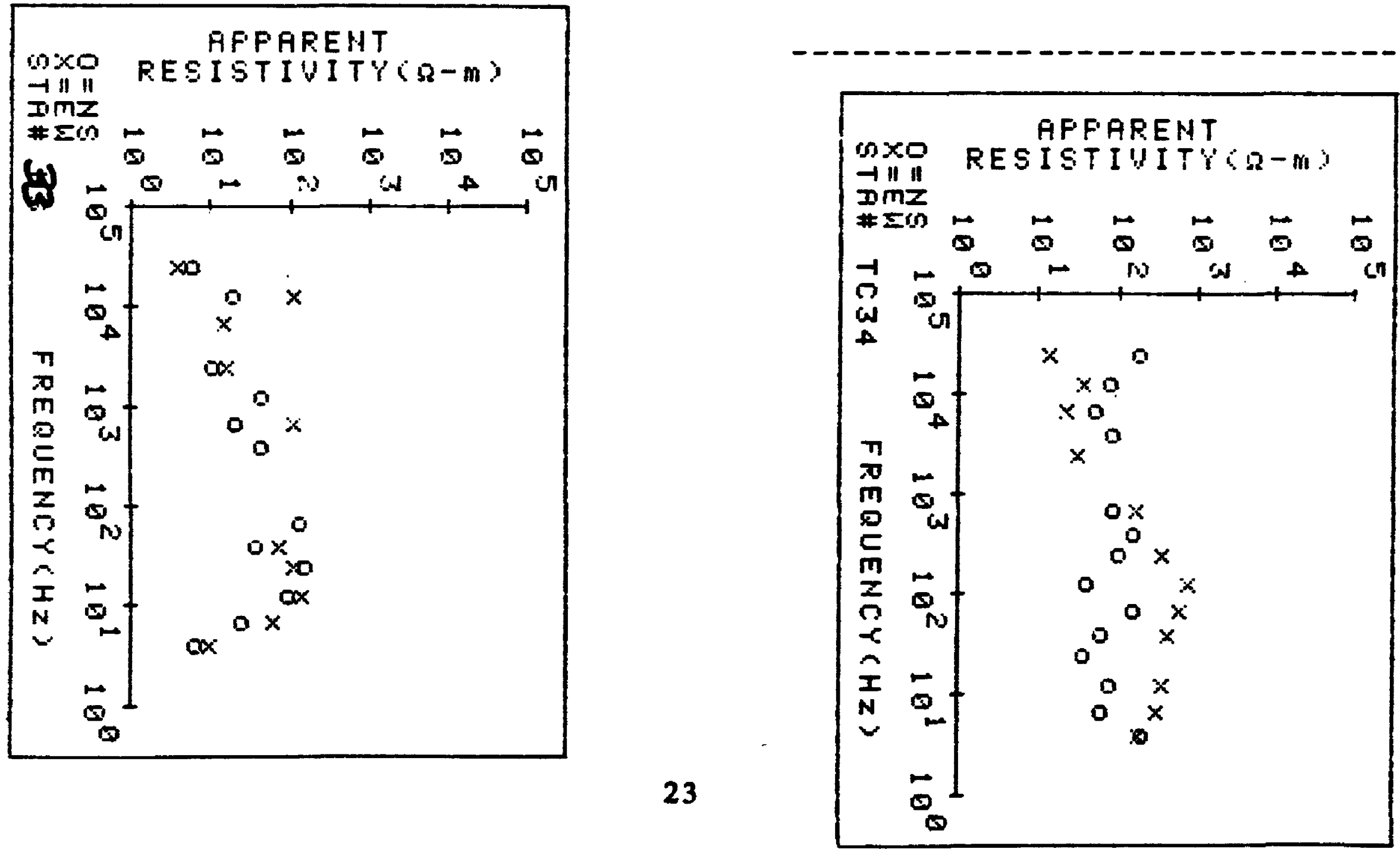
PROJ= JACKSON HOLE 94

STA-IDTC35F ILE NAME $=$ TC35

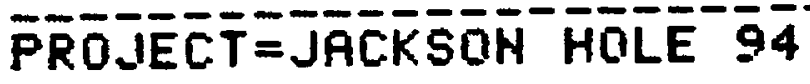

STA. ID_TC35 NS NO FREQ $=14$

$\begin{array}{rrcr}\text { FREQ } & \text { AP-RES } & \text { N OES } & \text { STD ERR } \\ 4.5 & 22.18 & 5 & 1.73 \\ 7.5 & 30.74 & 6 & 6.86 \\ 14.0 & 72.05 & 6 & 5.60 \\ 27.0 & 62.65 & 7 & 1.36 \\ 45.0 & 134.07 & 6 & 35.57 \\ 75.0 & 76.59 & 7 & 7.57 \\ 140.0 & 185.83 & 5 & 6.38 \\ 270.9 & 170.19 & 5 & 4.44 \\ 450.0 & 222.11 & 6 & 87.46 \\ 750.0 & 61.83 & 5 & 1.43 \\ 2700.0 & 35.96 & 5 & 2.22 \\ 4560.0 & 121.01 & 7 & 27.78 \\ 7500.0 & 112.91 & 3 & 12.33 \\ 4000.0 & 262.36 & 3 & 56.33\end{array}$

STR. ID_TC35 EW HO FREQ $=13$

$\begin{array}{rrcr}\text { FREQ } & \text { AP-RES } & \text { N OBS } & \text { STD ERR } \\ 4.5 & 384.12 & 8 & 121.09 \\ 7.5 & 200.22 & 3 & .79 \\ 14.0 & 99.76 & 6 & 8.09 \\ 27.0 & 106.69 & 7 & 2.71 \\ 45.0 & 232.12 & 6 & 19.24 \\ 270.0 & 157.40 & 5 & 2.15 \\ 450.0 & 79.85 & 8 & 19.17 \\ 750.6 & 94.98 & 6 & 19.61 \\ 2700.0 & 66.97 & 4 & 6.56 \\ 4590.0 & 85.87 & 6 & 5.79 \\ 7500.0 & 149.84 & 3 & 4.53 \\ 4990.0 & 122.47 & 3 & 7.99 \\ 7990.0 & 59.22 & 6 & 2.72\end{array}$

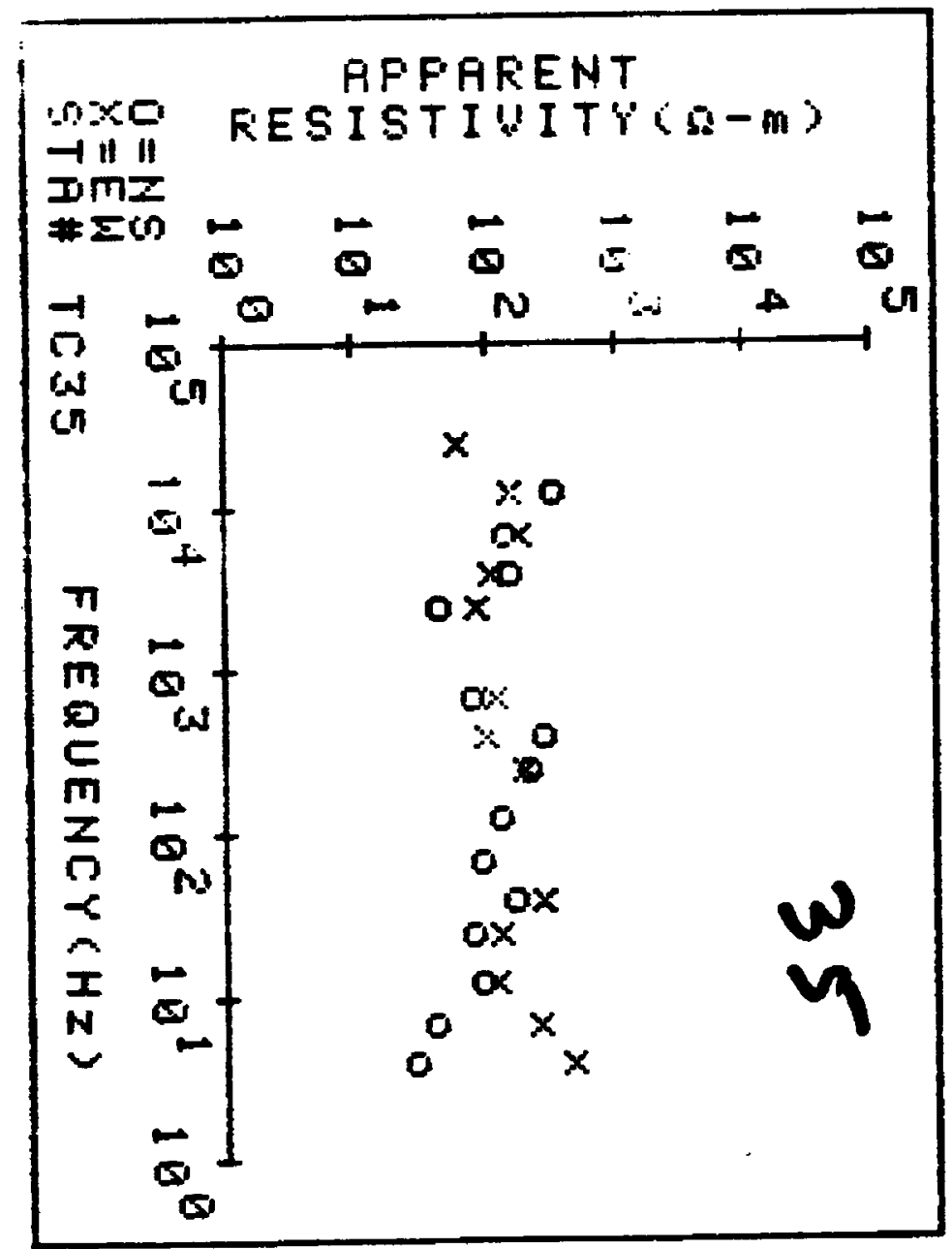

STA. ID_TC36 NS NO FREQ $=16$

\begin{tabular}{|c|c|c|c|}
\hline $\begin{array}{r}\text { FREQ } \\
4.5 \\
7.5 \\
14.0 \\
27.0 \\
45.0 \\
75.0 \\
140.0 \\
79.8 \\
450.0 \\
50.0 \\
109.0 \\
90.0 \\
500.0\end{array}$ & $\begin{array}{r}\text { AP-RES } \\
504.93 \\
2843.20 \\
724.18 \\
632.02 \\
1741.19 \\
1826.39 \\
489.85 \\
622.36 \\
487.43 \\
499.61 \\
242.46 \\
385.49 \\
63.59 \\
46.95 \\
5.17\end{array}$ & $\begin{array}{c}\text { N OBS } \\
8 \\
12 \\
7 \\
18 \\
14 \\
13 \\
19 \\
13 \\
18 \\
16 \\
19 \\
12 \\
16 \\
12 \\
16 \\
3\end{array}$ & $\begin{array}{r}\text { STO EF } \\
119.8 \\
680.6 \\
273.5 \\
139.7 \\
283.2 \\
202.8 \\
55.8 \\
65.3 \\
71.6 \\
93.4 \\
42.5 \\
73.7 \\
5.6 \\
7.0 \\
7.6\end{array}$ \\
\hline
\end{tabular}

STA. ID_TC36 EH NO FREQ $=16$

FREQ AP-RES N OBS STO ERR

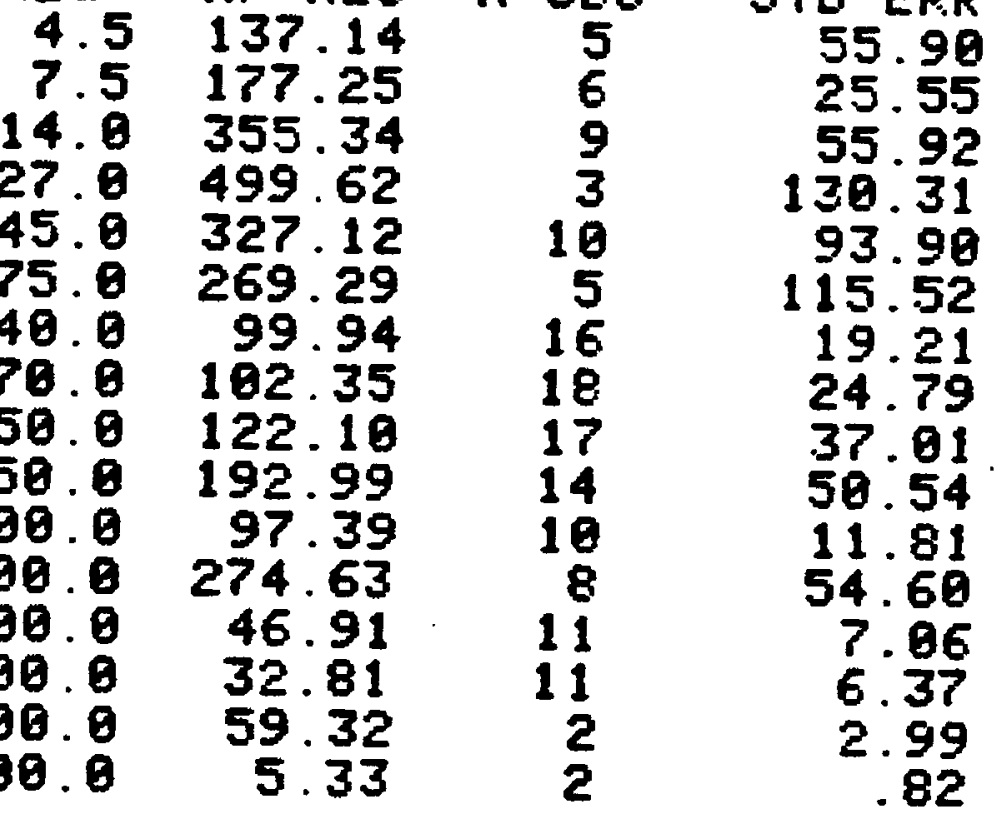

APPARENT

oxo RESISTIUITY(a-m)

$\operatorname{mmz}$

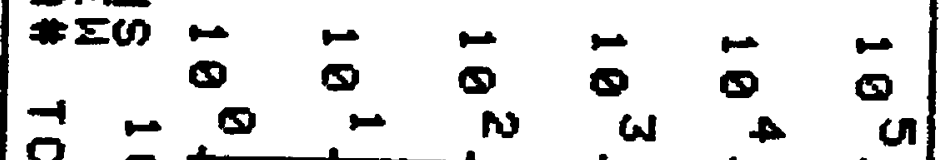

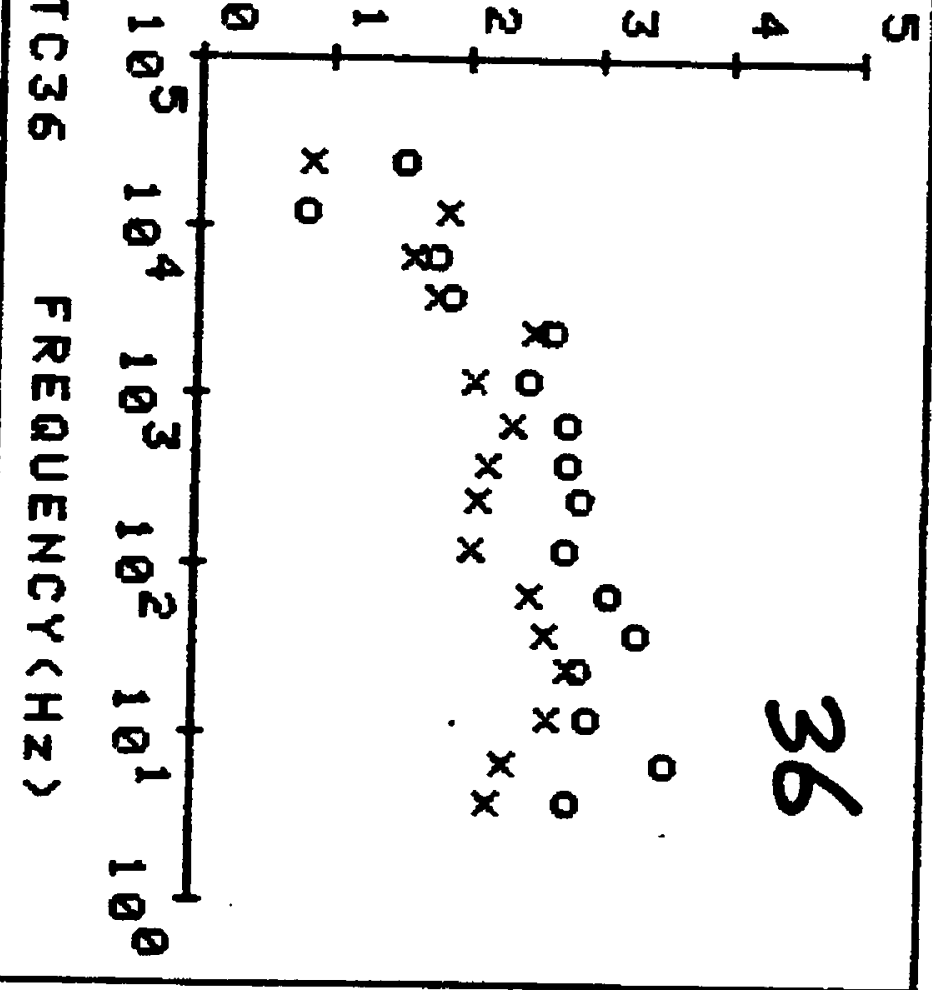


F'ROJECT =JACKKSON HOLE 94

STA. ID_TC37 NS NO FREQ $=13$

FREQ AP-RES N UBS STD ERR

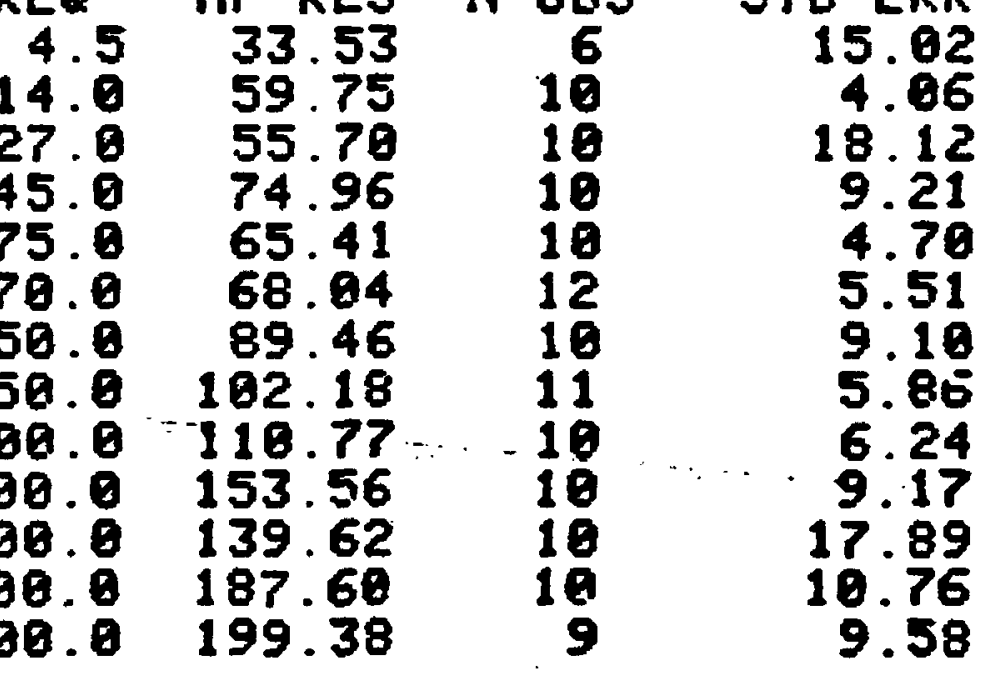

STA. ID_TC37 EN NO FREQ $=12$

FREQ AP-RES N OBS STD ERR

$\begin{array}{rrrr}4.5 & 7.10 & 5 & 3.51 \\ 14.0 & 18.45 & 8 & 3.22 \\ 27.0 & 14.99 & 8 & 5.95 \\ 45.0 & 27.89 & 9 & 3.63 \\ 75.0 & 31.91 & 12 & 1.60 \\ 70.0 & 48.02 & 10 & 6.87 \\ 50.0 & 71.39 & 10 & 7.66 \\ 50.0 & 44.57 & 18 & 4.97 \\ 60.9 & 32.05 & 9 & 5.09 \\ 90.0 & 31.58 & 10 & 9.97 \\ 80.0 & 42.13 & 8 & 7.11 \\ 00.0 & 67.83 & 3 & 4.23\end{array}$

14000.0

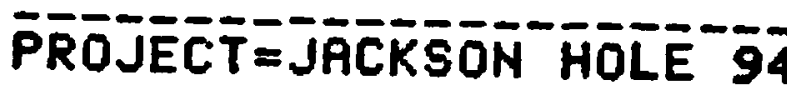

STR. ID_TC38 NS NO FREQ $=15$

FREQ AP-RES N OBS STD ERR

7.5155 .01

14.0250 .33

27.0255 .67

$45.0 \quad 716.80$

75.01467 .70

$146.0 \quad 993.04$

270.0698 .67

$450.6 \quad 581.55$

$750.8 \quad 622.53$

1480.0714 .60

2700.01241 .70

$4560.0 \quad 885.14$

7500.0227 .27

$14680.0 \quad 425.13$

$27000.0 \quad 73.91$

3
4
5
5
7
5
8
7
8
5
5
4
4
3
1

17.34

8.32

3.48

16.61

131.89

24.65

56.28

79.17

65.77

78.98

100.31

25.43

8.29

36.81

0.00

\section{STR. ID_TC38 EW NO FREQ $=14$}

FREQ AP-RES N OBS STD ERR

$14.0 \quad 134.12 \quad 4 \quad 6.61$

$27.0 \quad 175.67 \quad 5 \quad 47.55$

$\begin{array}{llll}45.8 & 221.89 & 5 & 42.31\end{array}$

$75.0 \quad 262.18 \quad 5 \quad 8.00$

$140.0 \quad 196.10 \quad 5 \quad 11.99$

$270.0 \quad 154.71 \quad 9 \quad: 81$

$\begin{array}{llll}450.6 & 365.27 & 9 & 20.40\end{array}$

$\begin{array}{llll}750.0 & 138.18 & 10 & 36.37\end{array}$

$\begin{array}{llll}1400.0 & 201.08 & 4 & 4.68\end{array}$

$\begin{array}{llll}2700.0 & 317.77 & 4 & 40.71\end{array}$

$\begin{array}{llll}4586.0 & 253.49 & 6 & 12.04\end{array}$

$\begin{array}{llll}7500.0 & 280.43 & 8 & 35.66\end{array}$

$\begin{array}{llll}14009 & 551.44 & 3 & 3.46\end{array}$

27060.0133 .971900
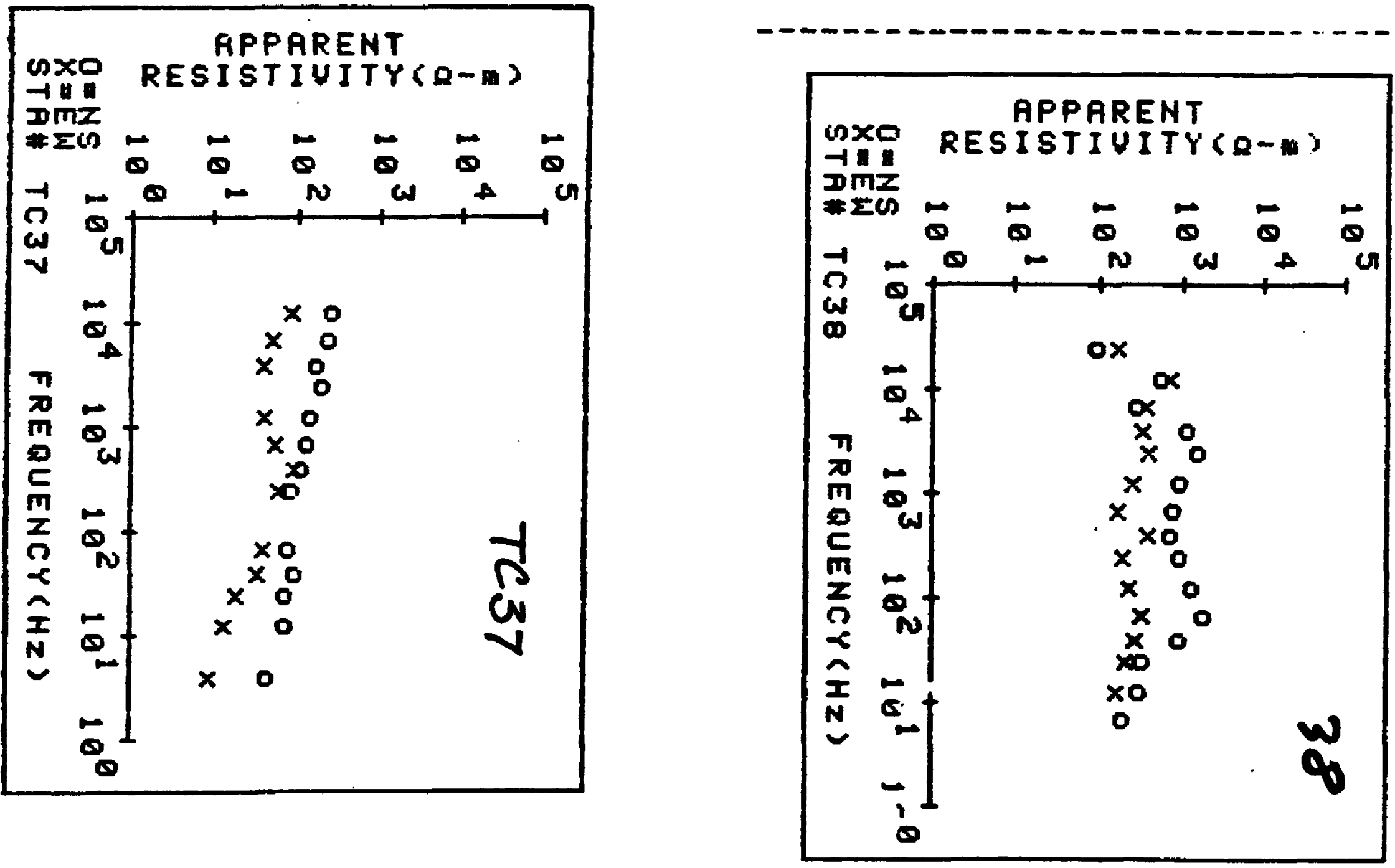
PROJ $=$ JACKSOH HOLE 94

STA-IDTC.39F ILE NAME $=$ TC.39

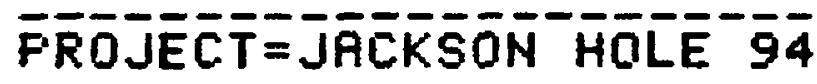

STA. ID_TC:39 NS NO FREC:= 9

$\begin{array}{rrcr}\text { FREQ } & \text { AP-RES } & \text { N DBS } & \text { STO ERR } \\ 4.5 & 189.12 & 7 & 38.78 \\ 7.5 & 106.85 & 8 & 18.31 \\ 140.0 & 95.05 & 8 & 59.35 \\ 270.0 & 82.65 & 9 & 7.63 \\ 450.0 & 165.29 & 6 & 19.34 \\ 490.0 & 311.04 & 3 & 11.61 \\ 2709.0 & 238.90 & 3 & 9.04 \\ 509.0 & 242.62 & 9 & 25.49 \\ 7690.6 & 121.72 & 4 & 8.41\end{array}$

STA. ID_TC39 EW NO FREQ $=11$

$\begin{array}{rrrr}\text { FREQ } & \text { AF-RES } & N \text { OBS } & \text { STD ERR } \\ 4.5 & 42.59 & 8 & 7.43 \\ 7.5 & 34.39 & 6 & 1.65 \\ 14.0 & 64.17 & 8 & 19.40 \\ 27.0 & 26.88 & 9 & 6.24 \\ 45.0 & 91.08 & 3 & 3.51 \\ 75.0 & 66.89 & 6 & 19.56 \\ 140.0 & 34.37 & 8 & 2.13 \\ 270.0 & 44.98 & 19 & 8.44 \\ 450.0 & 165.32 & 8 & 16.19 \\ 4909.0 & 205.41 & 3 & 6.49 \\ 7060.0 & 14.84 & 3 & .5\end{array}$

\begin{tabular}{|c|c|c|c|}
\hline $\begin{array}{r}\text { FREQ } \\
4.5 \\
7.5 \\
14.0 \\
27.0 \\
45.0 \\
75.0 \\
140.0 \\
270.0 \\
450.0 \\
750.0 \\
1400.0 \\
2700.0 \\
4500.0 \\
7500.0 \\
14000.0 \\
27000.0\end{array}$ & $\begin{array}{r}\text { AP-RES } \\
18.79 \\
34.13 \\
40.42 \\
37.65 \\
37.57 \\
71.53 \\
34.92 \\
54.14 \\
98.05 \\
81.44 \\
24.72 \\
15.92 \\
50.16 \\
67.68 \\
152.43 \\
36.10\end{array}$ & $\begin{array}{r}\text { N OES } \\
5 \\
8 \\
8 \\
8 \\
10 \\
8 \\
9 \\
18 \\
10 \\
4 \\
3 \\
5 \\
6 \\
8 \\
3 \\
1\end{array}$ & $\begin{array}{r}\text { STD ERR } \\
9.69 \\
7.45 \\
2 . \\
3.47 \\
14.23 \\
3.63 \\
4.64 \\
23.98 \\
27.69 \\
8.68 \\
7.89 \\
6.21 \\
7.62 \\
6.06\end{array}$ \\
\hline \multicolumn{2}{|c|}{ STA. ID_TC40 EH } & \multicolumn{2}{|c|}{ NO FREQ $=16$} \\
\hline $\begin{array}{r}\text { FREQ } \\
4.5 \\
7.5 \\
14.0 \\
27.0 \\
45.0 \\
75.0 \\
140.0 \\
270.0 \\
450.0 \\
750.0 \\
1400.0 \\
2700.0 \\
4500.0 \\
7500.0 \\
14000.9 \\
27000.0\end{array}$ & $\begin{array}{r}\text { AP-RES } \\
15.09 \\
7.04 \\
14.66 \\
22.11 \\
31.74 \\
65.61 \\
40.46 \\
51.45 \\
68.35 \\
37.90 \\
31.40 \\
34.36 \\
28.24 \\
165.27 \\
246.90 \\
167.00\end{array}$ & $\begin{array}{r}\text { N OBS } \\
3 \\
8 \\
5 \\
6 \\
9 \\
8 \\
10 \\
10 \\
8 \\
5 \\
5 \\
6 \\
3 \\
8 \\
3 \\
1\end{array}$ & $\begin{array}{r}\text { STD ERR } \\
1.17 \\
4.22 \\
6.81 \\
8.41 \\
6.89 \\
19.72 \\
6.08 \\
10.30 \\
15.09 \\
11.47 \\
3.44 \\
3.22 \\
14.29 \\
7.43 \\
0.90\end{array}$ \\
\hline
\end{tabular}

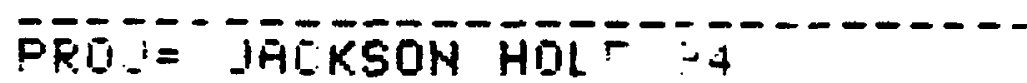

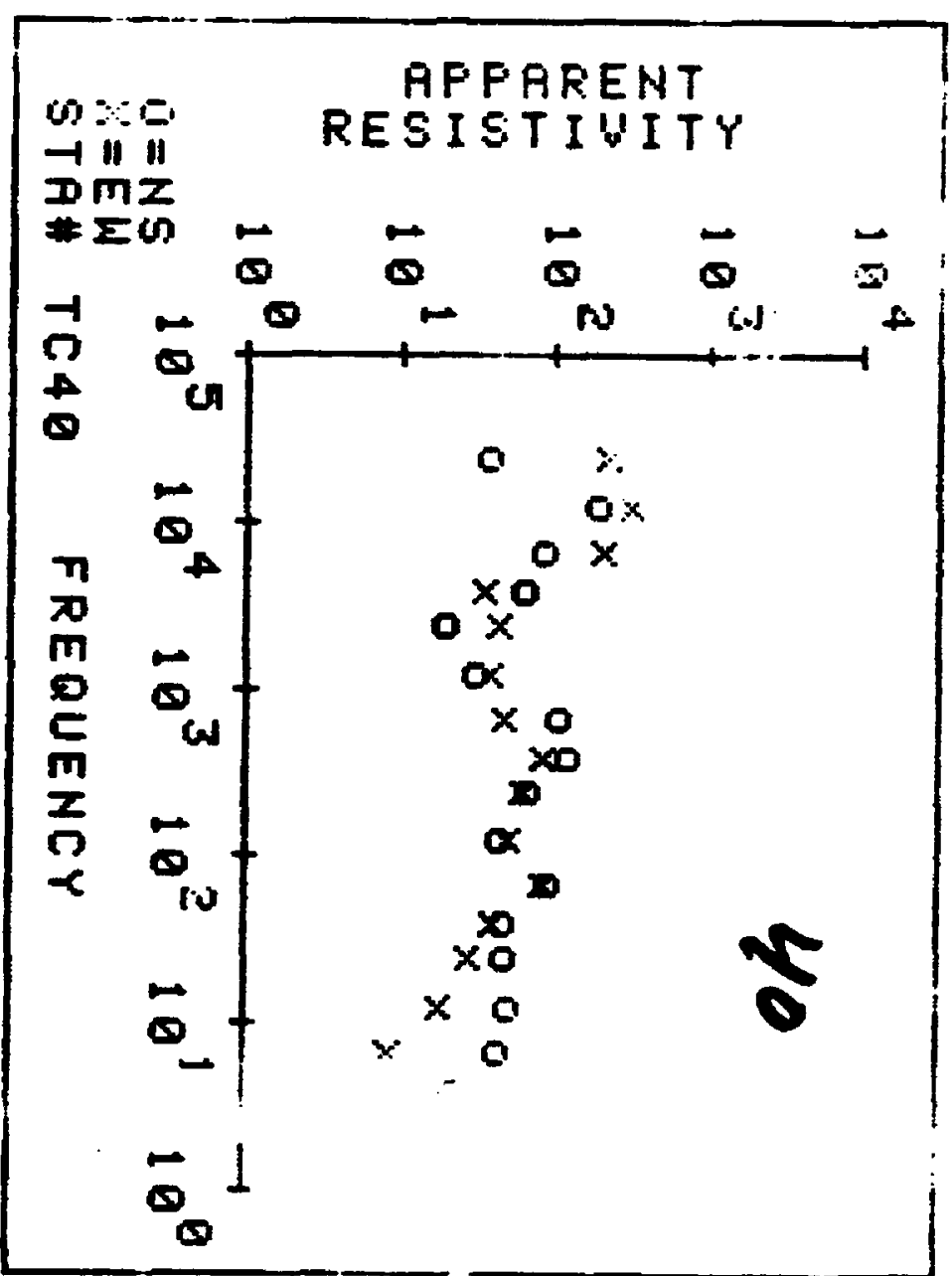


PROJI= JACKSIIH HOLE 94

STA-ILTC.41FILE NAME $=T C 41$

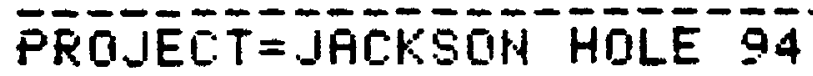

STA. ID_TC41 NS NO FREQ= 11

$\begin{array}{rrrr}\text { FREO } & \text { AF-PES } & \text { N OES } & \text { STI ERR } \\ 7.5 & 33.52 & 7 & 4.93 \\ 14.0 & 42.99 & 5 & 144.79 \\ 27.0 & 31.79 & 19 & 1.20 \\ 45.0 & 42.94 & 7 & 6.85 \\ 75.0 & 41.89 & 6 & 7.68 \\ 140.0 & 59.59 & 8 & 5.13 \\ 270.0 & 59.69 & 9 & 5.50 \\ 450.9 & 129.49 & 7 & 9.43 \\ 750.0 & 121.43 & 5 & 19.58 \\ 7590.0 & 75.94 & 7 & 8.26 \\ 4900.0 & 270.31 & 3 & 2.80\end{array}$

STA. ID_TR41 EW HO FREG $=11$

\begin{tabular}{|c|c|c|c|}
\hline 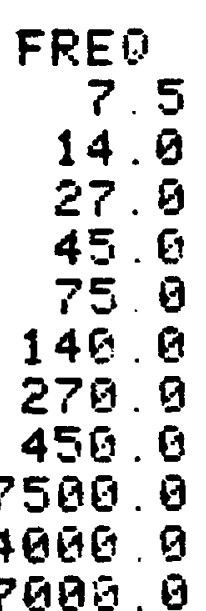 & $\begin{array}{r}A F-F E S \\
34.99 \\
35.28 \\
39.97 \\
38.49 \\
67.75 \\
56.97 \\
59.62 \\
164.93 \\
256.98 \\
415.49 \\
127.89\end{array}$ & 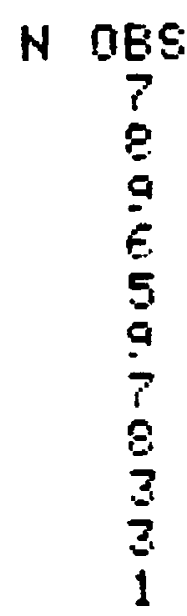 & $\begin{array}{r}\text { STD ERR } \\
1.99 \\
5.66 \\
3.91 \\
7.26 \\
2.39 \\
4.92 \\
2.92 \\
85.58 \\
21.28 \\
9.49 \\
0.00\end{array}$ \\
\hline
\end{tabular}

GFFAFENT

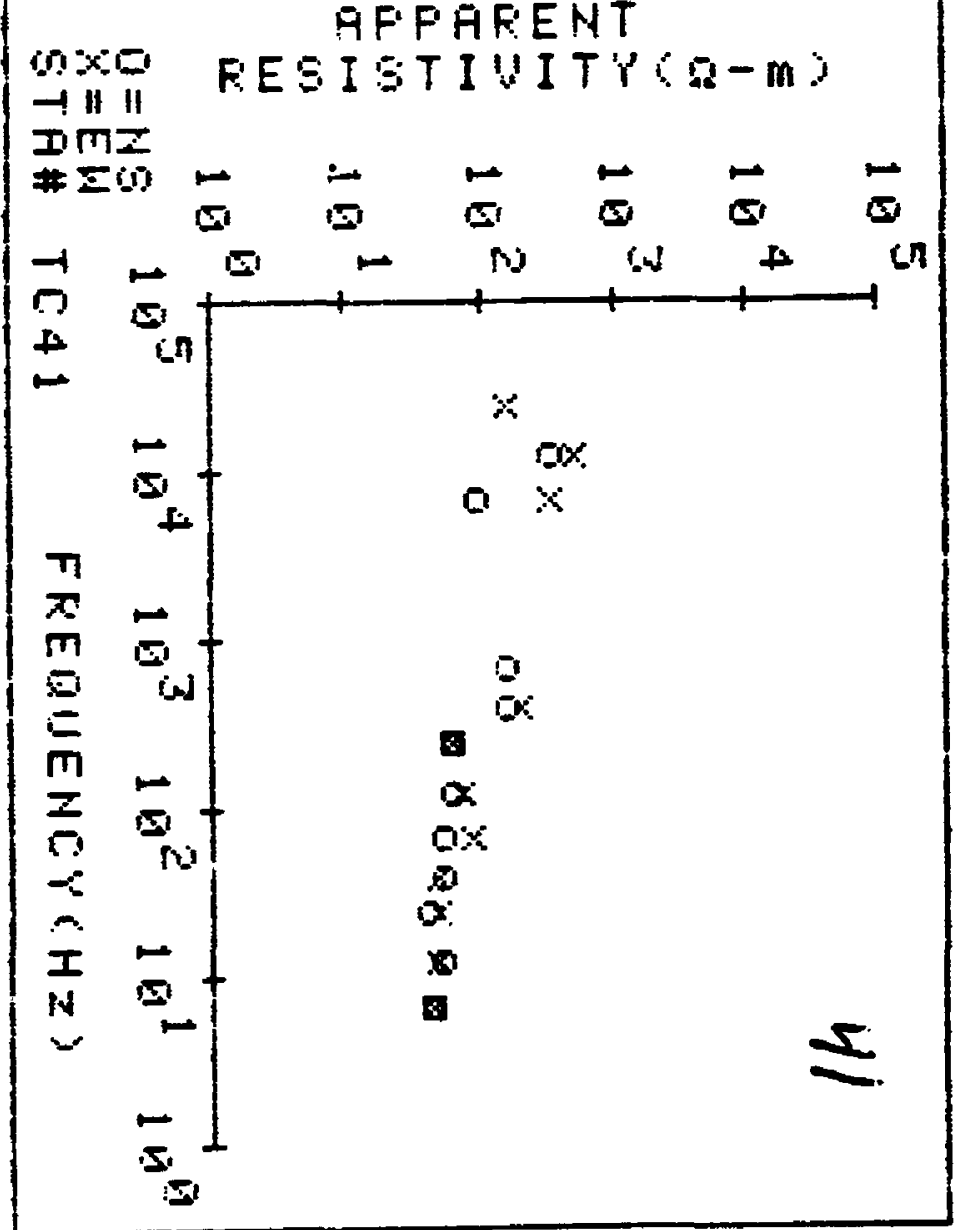

PROJ= JACKSON HOLE 94

STA-IUTC $42 F$ ILE NAME $=T C .42$

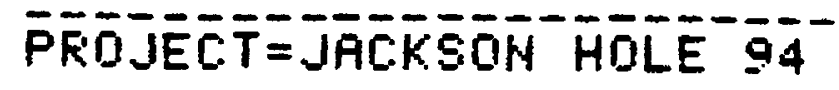

STA. ID_TC4Z NS HO FREQ= 13

FREQ AP-RES H ORS STL EFP. $4.5 \quad 60.35$ 2 16.58

$7.5 \quad 32.98 \quad 6 \quad 5.22$

$14.0 \quad 57.67 \quad 7 \quad 3.86$

$27.975 .12 \quad 10 \quad 5.92$

$45.9213 .77 \quad 7 \quad 14.42$

75.9 218.16 $7 \quad 42.64$

$140.9 \quad 449.34 \quad 19 \quad 135.31$

270.0192 .1073 .51

$450.0 \quad 397.94 \quad 5 \quad 63.23$

$750.0 \quad 701.63 \quad 5 \quad 17.92$

$2700.0 \quad 317.32 \quad 3 \quad 26.41$

$7506-1245.395522 .54$

$14090.0-1252-10 \quad 3 \quad 52.67$

STA. IOITC42 EW HO FREQ = 10

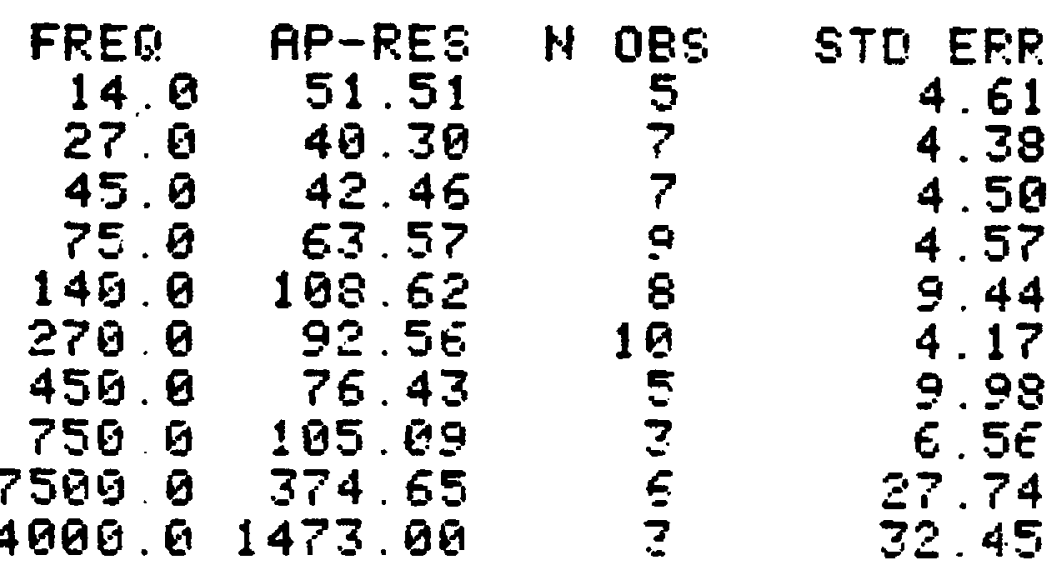

AFFARENT

RESISTIUITY $\Omega-m$ )

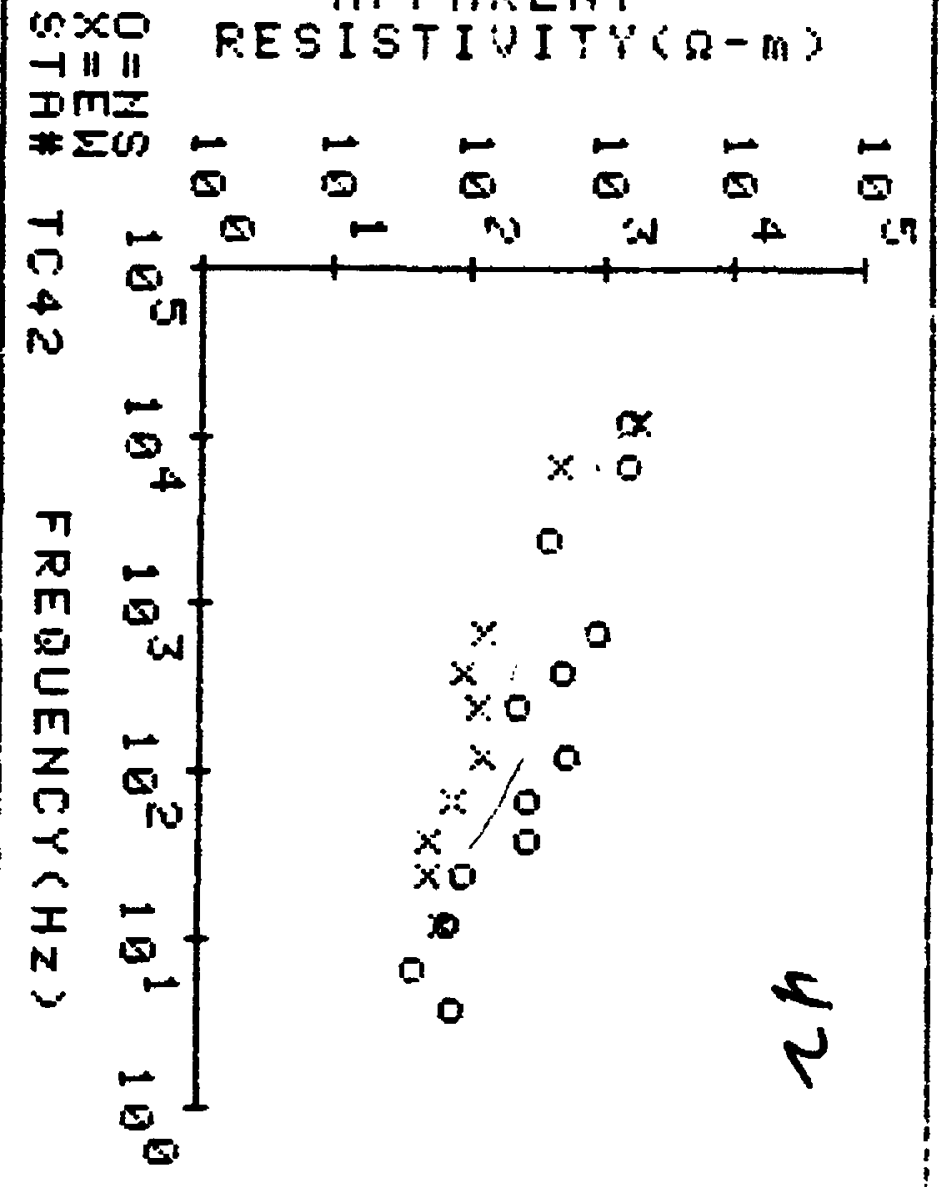


STA. ID_TC.43 NS NO FREQ $=15$

FREQ AP-RES $4.5 \quad 60.41$ $7.5 \quad 25.81$

$14.0 \quad 32.51$

27.9235 .16

45.0401 .47

75.9242 .18

$140.0 \quad 160.84$

$450.0 \quad 140.13$

750.0148 .73

1400.0151 .13

$2780.0 \quad 157.75$

$4500.0 \div 08.46$

$7500.0 \quad 272.03$

$14000.0 \quad 297.64$

27000.0183 .19

STA. ID_TC43 EN

FREQ AP-RES $4.5 \quad 236.80$

$7.5 \quad 17.50$

14.091 .90

27.0287 .00

$45.0 \quad 526.93$

$75.0 \quad 392.04$

$140.0 \quad 264.22$

270.0566 .47

$450.9 \quad 241.80$

$750.0 \quad 183.41$

1480.0283 .50

$2706.0 \quad 285.66$

$4500.0 \quad 185.82$

$7580.0 \quad 232.89$

$14000.0 \quad 255.95$

$\begin{array}{rr}\text { N OES } & \text { STD ERR } \\ 3 & 2.36 \\ 3 & 1.43 \\ 3 & 59.17 \\ 6 & 28.29 \\ 7 & 14.09 \\ 8 & 16.45 \\ 7 & 11.97 \\ 7 & 7.83 \\ 7 & 9.73 \\ 3 & 1.83 \\ 6 & 18.77 \\ 3 & 16.68 \\ 3 & 4.52 \\ 3 & \end{array}$

NO FREQ= 15

$\begin{array}{cr}N \text { OES } & \text { STD ERR } \\ 3 & 31.95 \\ 3 & .37 \\ 3 & 29.99 \\ 6 & 39.81 \\ 7 & 56.74 \\ 6 & 7.42 \\ 6 & 57.88 \\ 3 & 43.94 \\ 7 & 29.72 \\ 6 & 28.07 \\ 1 & 0.89 \\ 6 & 63.84 \\ 5 & 19.78 \\ 8 & 26.87 \\ 3 & 34.22\end{array}$

STA. IO_TC44 NS NO FREQ = 16

\begin{tabular}{|c|c|c|c|}
\hline $\begin{array}{r}\text { FREQ } \\
4.5 \\
7.5 \\
14.0 \\
27.0 \\
45.0 \\
75.0 \\
140.0 \\
270.0 \\
450.0 \\
750.0 \\
400.0 \\
700.0 \\
500.0 \\
500.0 \\
200.0 \\
980.0\end{array}$ & $\begin{array}{r}\text { AP-RES } \\
402.54 \\
246.11 \\
142.29 \\
461.42 \\
339.83 \\
367.41 \\
257.53 \\
98.69 \\
245.67 \\
73.36 \\
26.44 \\
83.14 \\
66.32 \\
62.16 \\
116.43 \\
16.79\end{array}$ & $\begin{array}{c}\text { OBS } \\
4 \\
8 \\
11 \\
7 \\
17 \\
16 \\
13 \\
16 \\
16 \\
20 \\
5 \\
6 \\
14 \\
10 \\
12 \\
7\end{array}$ & $\begin{array}{r}\text { STD ERR } \\
170.57 \\
36.92 \\
19.77 \\
59.18 \\
30.53 \\
33.05 \\
24.09 \\
10.39 \\
20.09 \\
5.33 \\
7.34 \\
16.98 \\
8.77 \\
5.12 \\
9.59 \\
.34\end{array}$ \\
\hline
\end{tabular}

STA. ID_TC44 EH NO FREQ $=16$

FREQ AP-RES $N$ OBS STD ERR $\begin{array}{llll}4.5 & 258.77 & 5 & 78.87\end{array}$

$\begin{array}{llll}7.5 & 139.02 & 8 & 45.37\end{array}$

$\begin{array}{llll}14.0 & 143.68 & 13 & 29.36\end{array}$

$\begin{array}{llll}27.0 & 211.79 & 18 & 50.05\end{array}$

$\begin{array}{llll}45.8 & 424.55 & 15 & 55.13\end{array}$

$\begin{array}{llll}75.9 & 422.28 & 11 & 40.39\end{array}$

$450.0 \quad 165.04 \quad 21 \quad 18.61$

$\begin{array}{llll}750.8 & 76.14 & 18 & 16.07\end{array}$

$\begin{array}{llll}1400.6 & 198.01 & 12 & 14.36\end{array}$

$2760.0 \quad 167.27 \quad 18$

$4500.0140 .00 \quad 15 \quad 18.24$

$7500.0 \quad 183.37 \quad 19021$

$14800.0 \quad 227.20$

27000.0311 .52
22.56

4.83

85.59

FROAJ JACKSON HOLE 94
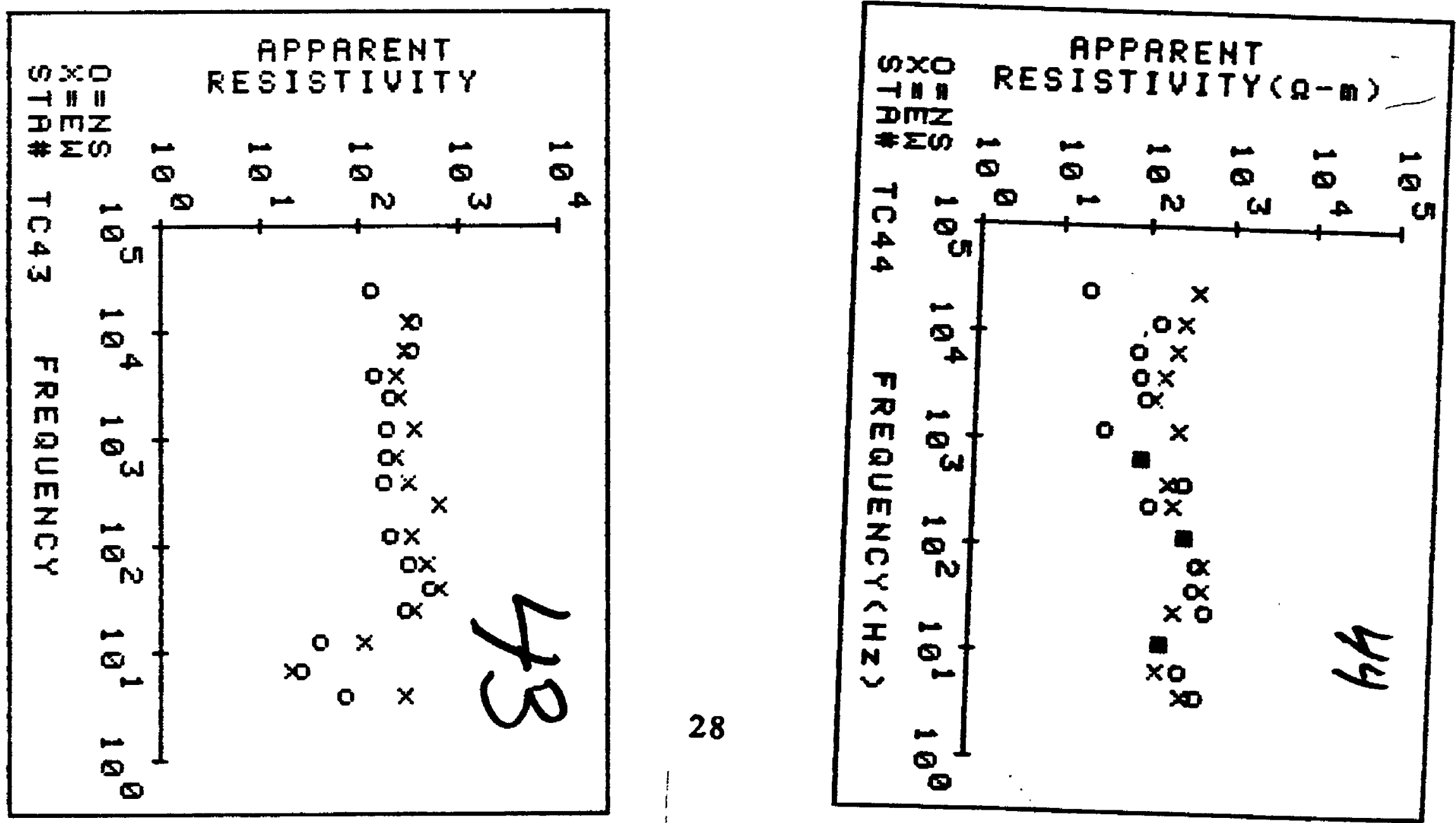
STA. ID_TC45 NS NO FREQ $=16$

\begin{tabular}{|c|c|c|c|}
\hline $\begin{array}{r}\text { FREQ } \\
4.5 \\
7.5 \\
14.0 \\
27.0 \\
45 . \theta \\
75.0 \\
148.0 \\
270.0 \\
458.0 \\
750.0\end{array}$ & $\begin{array}{l}\text { RP-RES } \\
114.34 \\
606.88 \\
462.32 \\
708.72 \\
798.97 \\
633.61 \\
443.93 \\
307.35 \\
327.71 \\
270.11 \\
295.66 \\
240.16 \\
263.75 \\
314.86 \\
368.91 \\
410.77\end{array}$ & $\begin{array}{c}\text { N OBS } \\
8 \\
16 \\
4 \\
19 \\
18 \\
24 \\
6 \\
61 \\
21 \\
13 \\
17 \\
6 \\
11 \\
16 \\
12\end{array}$ & $\begin{array}{r}\text { STD ERR } \\
66.68 \\
122.99 \\
109.08 \\
126.49 \\
34.62 \\
26.45 \\
54.97 \\
28.41 \\
19.30 \\
12.35 \\
6.76 \\
18.59 \\
63.42 \\
121.61 \\
9.09 \\
0.00\end{array}$ \\
\hline
\end{tabular}

STA. ID_TC45 EW NO FREQ $=16$

FREQ AF-RES N OBS STD ERP

$4.5 \quad 139.31$

7.561 .07

14.0119 .26

27.0181 .03

$45.0 \quad 241.57$

$75.0 \quad 242.57$

149.0171 .43

270.0128 .25

450.158 .01

750.9119 .04

1400.9

2700.0

4500.0

7596.0

14989.8

118.79

115.68

295.63

235.58

707.46

27000.019 .25

2
11
16
18
11
18
18
21
23
22
6
11
20
29
2
1

21.33

6.46

19.81

31.95

26.44

16.83

5.86

20.17

5.35

4.87

8.63

10. 30

6.51

101.79

0. 60
4.71
STA. ID_TC46 NS NO FREQ $=16$

\begin{tabular}{|c|c|c|c|}
\hline $\begin{array}{r}\text { FREQ } \\
4.5 \\
7.5 \\
14.0 \\
27.0 \\
45.0 \\
75.0 \\
140.0 \\
270.0 \\
450.0 \\
750.0 \\
1460.0 \\
2700.0 \\
4509.0 \\
7500.0\end{array}$ & $\begin{array}{l}\text { RP-RES } \\
333.75 \\
298.79 \\
339.19 \\
325.69 \\
283.65 \\
315.94 \\
174.80 \\
116.68 \\
217.62 \\
141.52 \\
225.39 \\
167.32 \\
157.46 \\
178.49 \\
279.11 \\
16.16\end{array}$ & $\begin{array}{c}\text { OBS } \\
4 \\
10 \\
10 \\
9 \\
12 \\
11 \\
10 \\
12 \\
11 \\
11 \\
4 \\
5 \\
11 \\
10\end{array}$ & $\begin{array}{r}\text { STD ERR } \\
13.92 \\
32.18 \\
72.17 \\
59.31 \\
42.28 \\
28.55 \\
5.95 \\
8.53 \\
10.66 \\
18.43 \\
17.19 \\
6.83 \\
15.18 \\
18.77 \\
7.98 \\
3.12\end{array}$ \\
\hline
\end{tabular}

STA. ID_TC46 EW NO FREQ $=15$

FREQ AP-RES N OBS STD EFR $4.5 \quad 3.51 \quad 6 \quad 33$

$\begin{array}{llll}7.5 & 133.79 & 6 & 13.86\end{array}$

$14.0 \quad 192.82 \quad 11 \quad 16.84$

$\begin{array}{llll}27.8 & 151.61 & 9 & 11.18\end{array}$

$\begin{array}{llll}45.0 & 211.85 & 16 & 17.57\end{array}$

$\begin{array}{llll}75.0 & 91.29 & 10 & 6.38\end{array}$

$\begin{array}{llll}148.6 & 163.68 & 12 & 12.11\end{array}$

$\begin{array}{llll}270.0 & 164.63 & 12 & 8.38\end{array}$

$\begin{array}{llll}450.9 & 128.78 & 16 & 15.75\end{array}$

$750.0 \quad 111.23 \quad 10 \quad 15.12$

$\begin{array}{llll}2700.0 & 120.86 & 8 & 6.87\end{array}$

$\begin{array}{llll}4560.8 & 142.14 & 12 & 21.92\end{array}$

$\begin{array}{llll}7500.0 & 373.35 & 10 & 44.94\end{array}$

$\begin{array}{rrrr}14000.0 & 302.05 & 4 & 3.14 \\ 27000.0 & 201.23 & 2 & 99.04\end{array}$

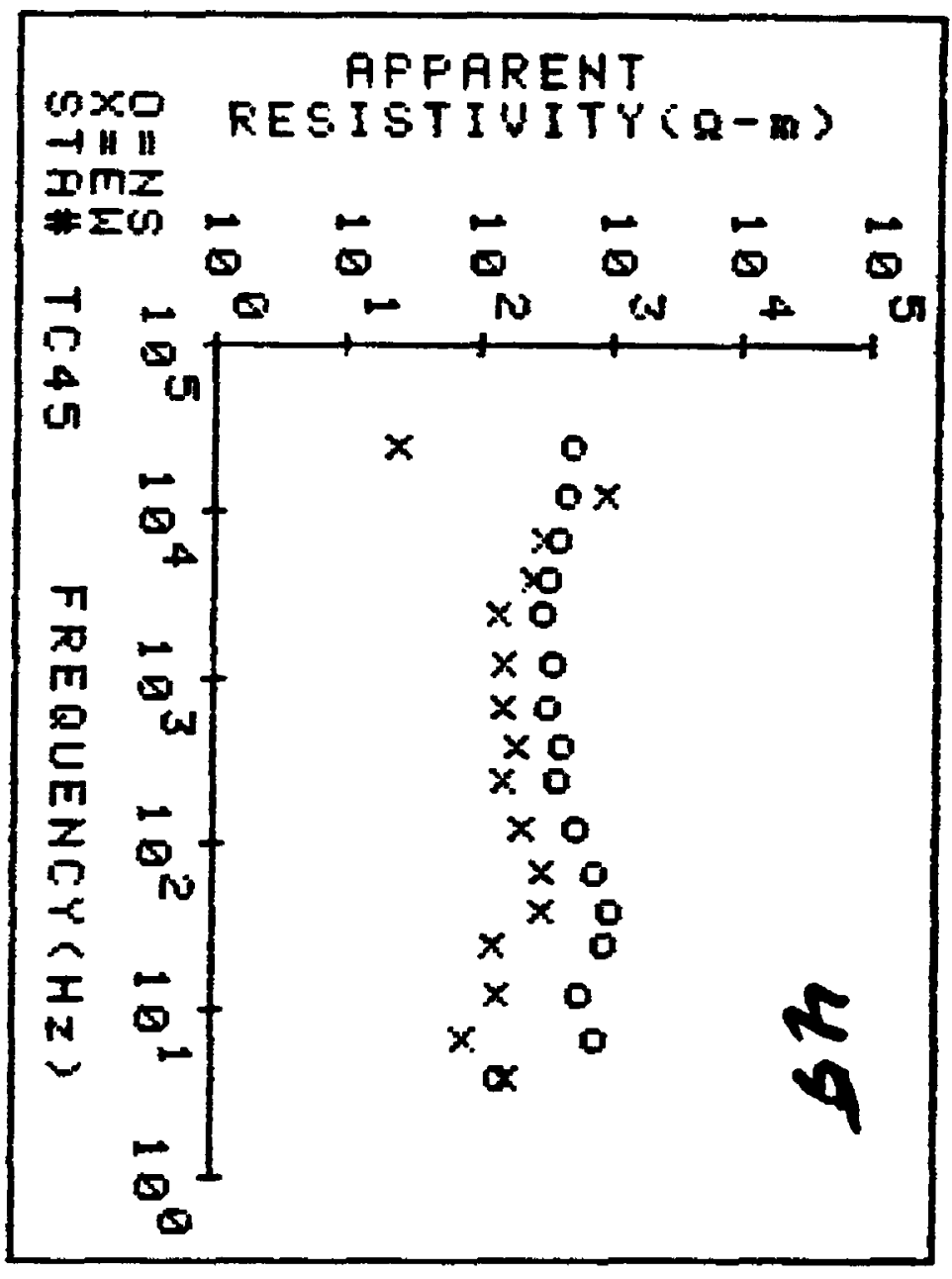

29

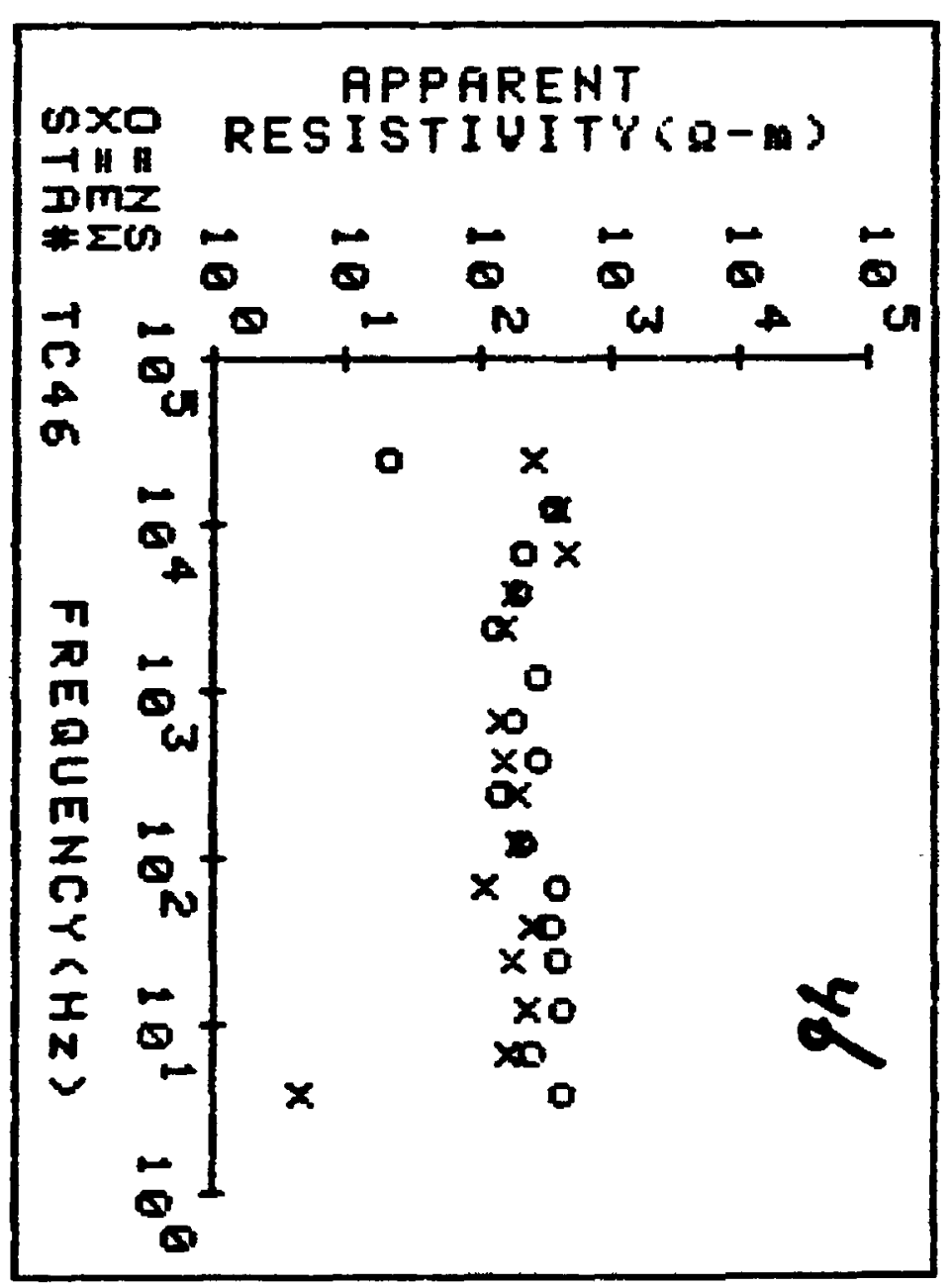


PROJ = JACKSON HOLE 94

STA-IDTC 48 FILE NAME $=$ TC 48

FROJJECT =JACKSONON HOLE 94

STA. ID_TC.47 NS NO FREQ $=14$

FREQ AP-RES N DBS STD ERR

4.531 .00

14.0197 .60

$27.0 \quad 359.00$

45.9392 .90

75.0339 .00

$270.0 \quad 170.00$

$450.9 \quad 296.87$

750.0172 .10

$1400.0 \quad 114.98$

$2700.9 \quad 156.91$

4.500 .0

7500.0

14000.0

181.09

76.90

108.52

62.50

3
9
10
10
10
10
10
10
10
10
10
3
8

49.00

39.00

33.90

27.00

22.90

18.98

7.15

2.94

12.41

23.86

19.29

11.58

6.84
13.90

STA. ID_TC.47 EW NO FREQ $=12$

\begin{tabular}{|c|c|c|c|}
\hline $\begin{array}{r}\text { FREQ } \\
4.5 \\
14.0 \\
27.0 \\
45.0 \\
75.0 \\
270.9 \\
450.0 \\
2790.0 \\
7590.0 \\
7590.0 \\
4600.0 \\
7990.0\end{array}$ & $\begin{array}{r}\text { AP-RES } \\
14.53 \\
25.46 \\
65.43 \\
128.52 \\
118.86 \\
83.53 \\
195.95 \\
57.73 \\
85.79 \\
113.40 \\
82.36 \\
22.38\end{array}$ & $\begin{array}{c}\text { NOBS } \\
3 \\
4 \\
6 \\
7 \\
7 \\
16 \\
16 \\
8 \\
11 \\
\frac{1}{3} \\
3 \\
3\end{array}$ & $\begin{array}{r}\text { STD ERR } \\
1.98 \\
4.67 \\
21.14 \\
20.57 \\
25.16 \\
10.94 \\
12.98 \\
11.44 \\
9.74 \\
12.26 \\
3.65 \\
.85\end{array}$ \\
\hline
\end{tabular}

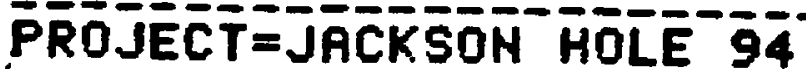

STA. ID_TC48 NS NO FREQ $=14$ FREQ AP-RES $N$ OBS STD $14.0 \quad 91.66$

27.0677 .01

45.0435 .51

$75.0 \quad 382.29$

140.0206 .33

$270.0 \quad 149.48$

$450.0 \quad 294.19$

$750.0 \quad 299.96$

$2700.0 \quad 415.47$

$4500.0 \quad 233.90$

7500.062 .31

14900.0258 .46

27000.021 .13

3
4
8
10
7
6
7
9
8
5
10
4
3
3

8.13

35.19

61.17

61.54

12.06

64.15

8.03

52.38

26.45

124.97

25.17

8.66

125.10

8.57

STA. ID_TC48 EH NO FREQ $=15$

FREQ AP-RES H OBS STD ERR

$4.5 \quad 60.77$

$7.5 \quad 212.81$

14.0162 .56

27.0185 .56

45.0360 .08

$75.0 \quad 411.80$

$140.0 \quad 114.89$

$270.0 \quad 71.66$

450.0101 .16

$750.0 \quad 164.87$

$2709.0 \quad 98.52$

$4500.0 \quad 76.01$

$7500.0 \quad 204.29$

14600.0219 .62

$27000.0 \quad 91.68$

3
3
3
3
8
8
8
7
9
9
9
6
6
3
3
3

11.04

65.06

33.47

34.04

22.39

109.21

5.59

6.85

9.04

6.97

2.17

20.19

25.50

1.64
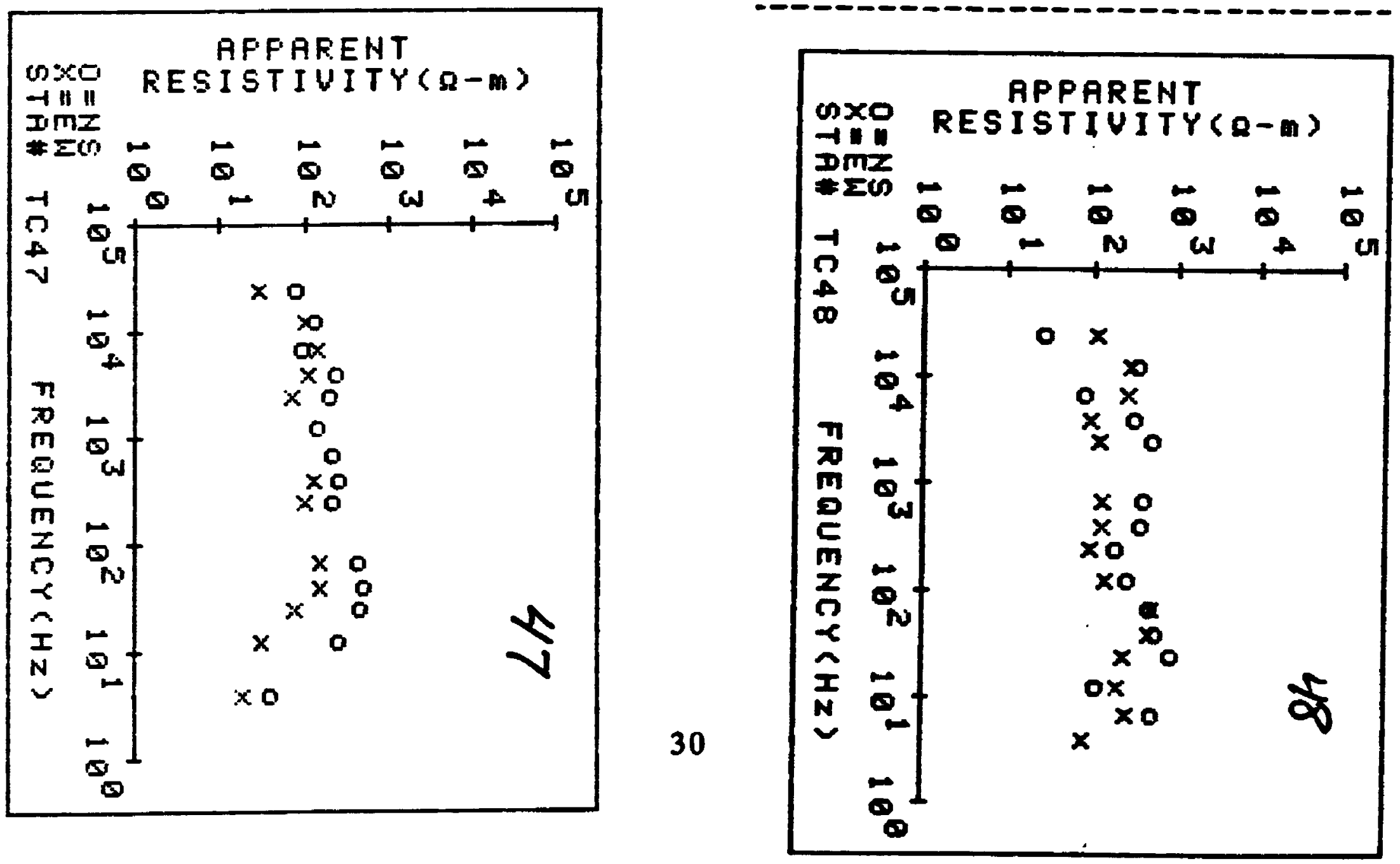
STA. ID_TC:49 NS NO FREQ $=12$

\begin{tabular}{|c|c|c|c|}
\hline $\begin{array}{r}\text { FREQ } \\
14.0 \\
27.0 \\
45.0 \\
75.0 \\
140.0 \\
279.0 \\
450.0 \\
750.0 \\
490.0 \\
790.0 \\
500.0 \\
990.9\end{array}$ & $\begin{array}{r}\text { AP-RES } \\
39.55 \\
275.11 \\
232.33 \\
174.12 \\
153.24 \\
193.55 \\
161.47 \\
144.01 \\
172.72 \\
497.41 \\
389.68 \\
166.18\end{array}$ & $\begin{array}{c}\text { OBS } \\
3 \\
8 \\
7 \\
8 \\
9 \\
8 \\
9 \\
8 \\
7 \\
8 \\
8 \\
3\end{array}$ & $\begin{array}{r}\text { STD ERR } \\
4.95 \\
64.92 \\
95.21 \\
23.11 \\
11.61 \\
19.89 \\
14.65 \\
16.11 \\
14.85 \\
112.62 \\
36.34 \\
54.69\end{array}$ \\
\hline
\end{tabular}

STA. ID_TC49 EW HO FREQ $=12$

FREQ AP-RES N OBS STD ERR

$\begin{array}{rrrr}14.9 & 2.74 & 3 & .97 \\ 45.0 & 4.58 & 3 & .14 \\ 140.0 & 16.95 & 8 & 2.37 \\ 270.0 & 13.02 & 8 & 2.62 \\ 450.0 & 84.59 & 8 & 93.83 \\ 550.9 & 7.39 & 4 & .73 \\ 409.0 & 48.21 & 7 & 7.76 \\ 790.9 & 11.64 & 7 & 4.33 \\ 590.0 & 57.33 & 8 & 5.72 \\ 990.0 & 125.93 & 8 & 20.97 \\ 900.0 & 230.34 & 3 & 4.28 \\ & 6.43 & 1 & 0.00\end{array}$

27090.0
PROJ= JACKSON HOLE 94

STA-IDTC5OF ILE HAME $=T C 50$

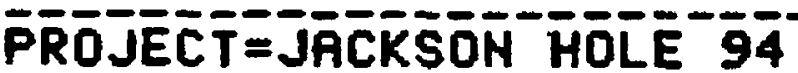

STA. ID_TC5O NS NO FREQ $=9$

FREQ AP-RES N OBS STD ERR

$\begin{array}{llll}27.8 & 134.09 & 3 & 10.48\end{array}$

$\begin{array}{llll}45.0 & 222.80 & 4 & 7.59\end{array}$

$75.8 \quad 540.54 \quad 4 \quad 22.09$

140.0203 .36

.270 .0248 .25

$4500.8 \quad 121.21$

$7500.0 \quad 150.10$

14800.8186 .73

27000.061 .84

2.58

23.80

4.75

11.55 .

1.98

3.35

STA. ID_TC5O EH NO FREQ $=11$

FREQ AP-RES

14.81181 .68

27.8534 .61

$45.8 \quad 267.53$

$75.8 \quad 372.10$

$140.6 \quad 145.34$

270.0687 .42

$456.8 \quad 173.47$

750.0162 .39

$2786.0 \quad 61.83$

14008.6258 .34

$27800.8 \quad 81.42$

$\begin{array}{cr}\text { N OBS } & \text { STD ERR } \\ 2 & 41.97 \\ 5 & 29.71 \\ 5 & 92.60 \\ 6 & 65.57 \\ 3 & 7.39 \\ 3 & 13.14 \\ 3 & 17.57 \\ 5 & 32.86 \\ 6 & 21.88 \\ 4 & 7.54 \\ 3 & 5.79\end{array}$

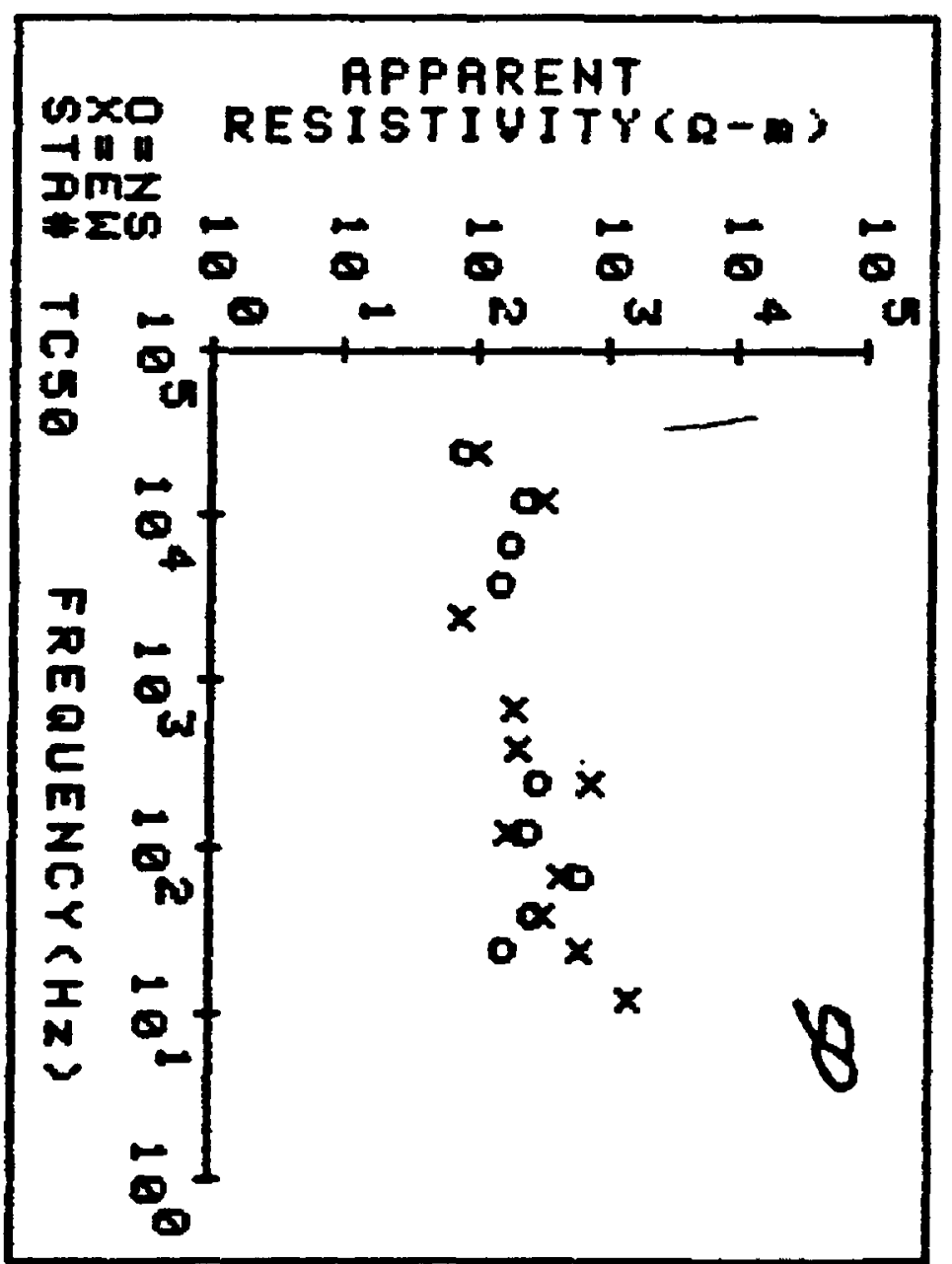


$\bar{P} \overline{R O J E} \bar{C} \bar{T}=\bar{J} \bar{C} \bar{C} \bar{S} \bar{N}$ HOLE 94

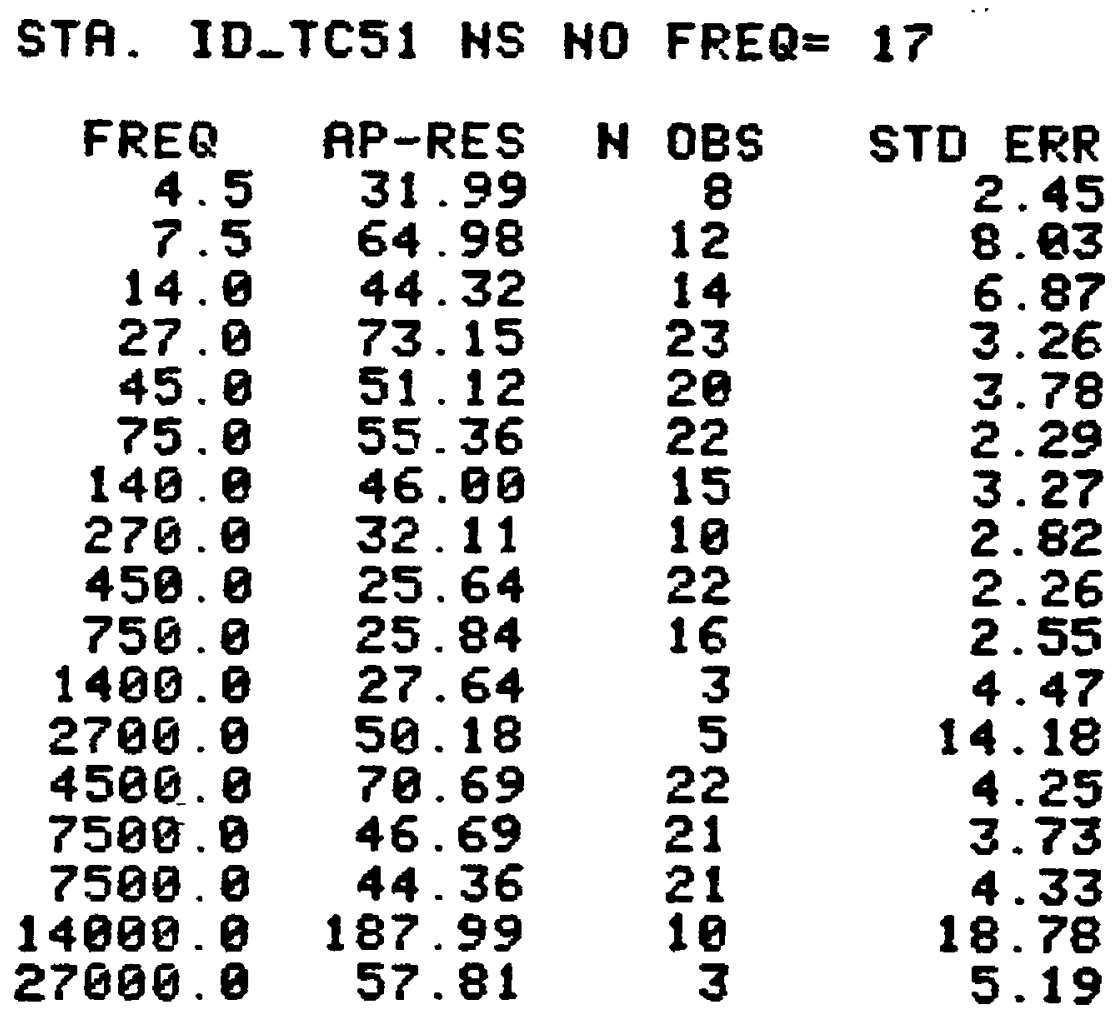

STA. ID_TC51 EW NO FREQ $=16$

\begin{tabular}{|c|c|c|c|c|}
\hline $\begin{array}{r}\text { FREQ } \\
4.5 \\
7.5 \\
14.0 \\
27.0 \\
45.0 \\
75.0 \\
140.0 \\
276.0 \\
450.0 \\
750.0 \\
400.0 \\
700.0 \\
500.0\end{array}$ & $\begin{array}{r}\text { AP-RES } \\
74.68 \\
35.38 \\
22.33 \\
57.31 \\
17.57 \\
40.91 \\
66.38 \\
47.14 \\
52.62 \\
30.94 \\
24.49 \\
66.82 \\
75.68 \\
169.82 \\
211.26 \\
44.66\end{array}$ & $\mathbf{N}$ & $\begin{array}{c}\text { OBS } \\
5 \\
12 \\
16 \\
13 \\
14 \\
19 \\
14 \\
10 \\
21 \\
13 \\
3 \\
6 \\
21 \\
11 \\
3 \\
1\end{array}$ & $\begin{array}{r}\text { STD ERR } \\
122.24 \\
4.52 \\
6.88 \\
4.13 \\
5.81 \\
7.14 \\
8.78 \\
7.88 \\
4.76 \\
7.83 \\
8.25 \\
18.89 \\
6.13 \\
6.74 \\
24.84 \\
0.80\end{array}$ \\
\hline
\end{tabular}

PROJ = JACKSON HOLE 94

STA-IDTCSZFILE NAME $=$ TC52

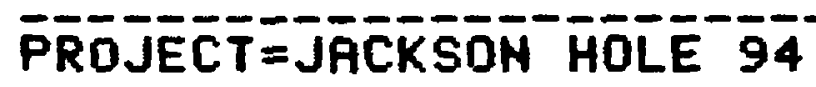

STA. ID_TCS2 NS NO FREQ= 9

FREQ AP-RES N OBS STD ERR

$\begin{array}{rrrr}4.5 & 72.49 & 3 & 2.69 \\ 14.79 & 17.79 & 6 & \end{array}$

$27.0 \quad 20.90 \quad 5 \quad 1.35$

$\begin{array}{llll}75.8 & 51.80 & 6 & 1.62\end{array}$

$146.0 \quad 112.68 \quad 3 \quad 5.69$

$\begin{array}{llll}270.8 & 194.69 & 6 & 3.16\end{array}$

$2700.9 \quad 864.65 \quad 3 \quad 126.42$

$\begin{array}{rrrr}4500.0 & 338.32 & 3 & 13.39 \\ 7500.9 & 986.93 & 3 & 128.10\end{array}$

STA. ID_TCS2 EH NO FREQ $=6$

FREQ AP-RES $N$ OES STO ERR

$\begin{array}{llll}27.0 & 137.59 & 3 & 20.76\end{array}$

$\begin{array}{llll}75.0 & 144.87 & 7 & 1.29\end{array}$

$\begin{array}{llll}270.0 & 252.64 & 5 & 5.17\end{array}$

$750.01600 .90 \quad 4 \quad 14.79$

$\begin{array}{rrrr}4500.0 & 1670.50 & 5 & 12.35 \\ 7500.0 & 5073.50 & 5 & 218.22\end{array}$

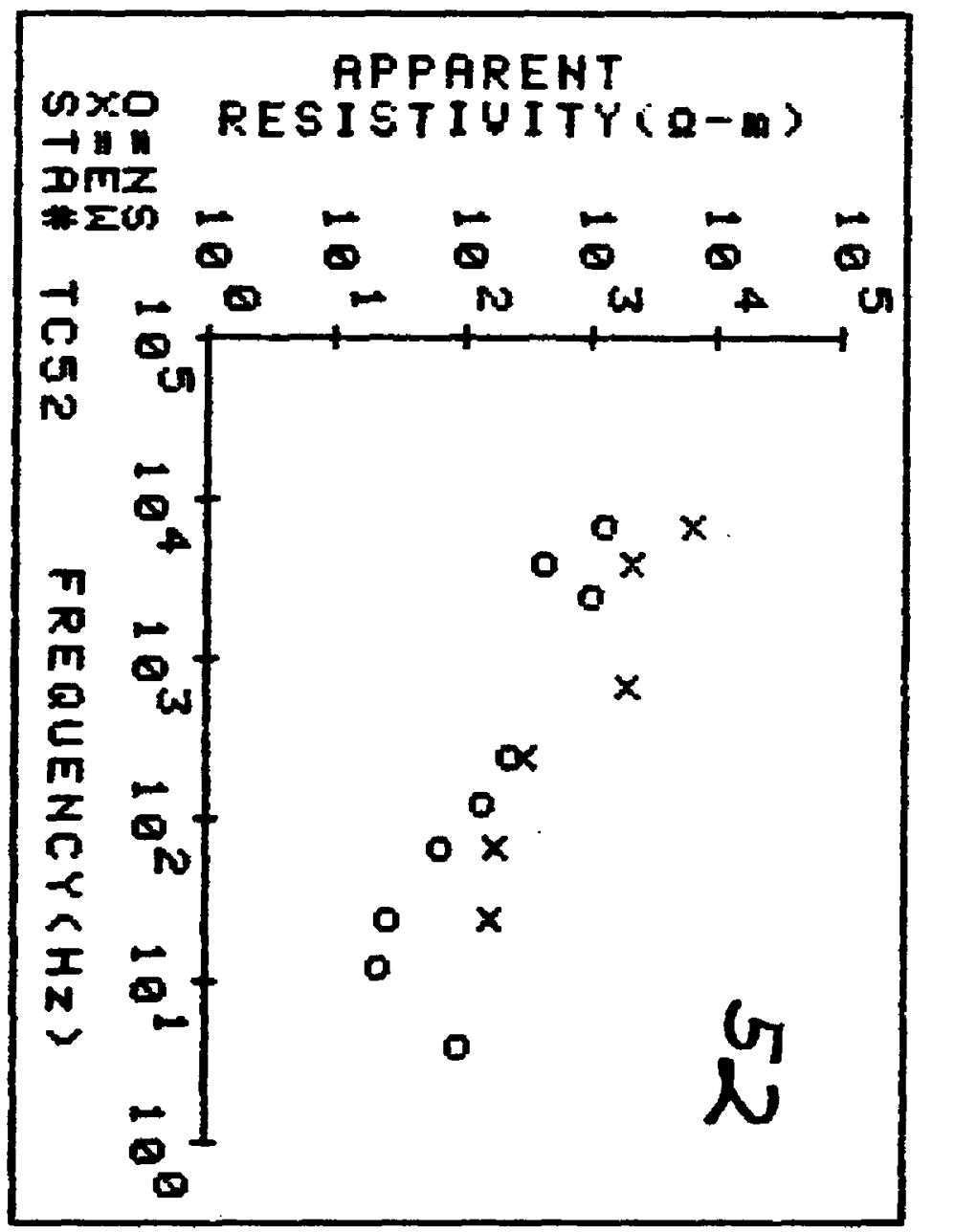


PROJ= JACKSOH HOLE 94

STA-IUTC53FILE NAME $=$ TC53

PRROJECTT=JACKSON HOLE 94

STA. ID_TC53 NS NO FREQ $=14$

\begin{tabular}{|c|c|c|c|}
\hline $\begin{array}{r}\text { FREQ } \\
7.5 \\
14.0 \\
27.0 \\
45.0 \\
75.0 \\
148.0 \\
270.0 \\
456.0 \\
758.0 \\
408.0\end{array}$ & $\begin{array}{r}\text { AP-RES } \\
35.84 \\
33.89 \\
46.72 \\
42.53 \\
78.81 \\
87.13 \\
98.87 \\
189.84 \\
159.11 \\
186.35 \\
126.56 \\
313.13 \\
182.23 \\
59.51\end{array}$ & $\begin{array}{c}\text { N OBS } \\
3 \\
4 \\
6 \\
9 \\
9 \\
7 \\
7 \\
8 \\
8 \\
5 \\
3 \\
5 \\
9 \\
3 \\
1\end{array}$ & $\begin{array}{r}\text { STD ERR } \\
.46 \\
2.18 \\
2.91 \\
2.41 \\
2.54 \\
6.34 \\
16.82 \\
39.12 \\
1.59 \\
10.67 \\
15.66 \\
11.66\end{array}$ \\
\hline
\end{tabular}

STA. ID_TC53 EW HO FREQ $=14$

\begin{tabular}{|c|c|c|c|}
\hline 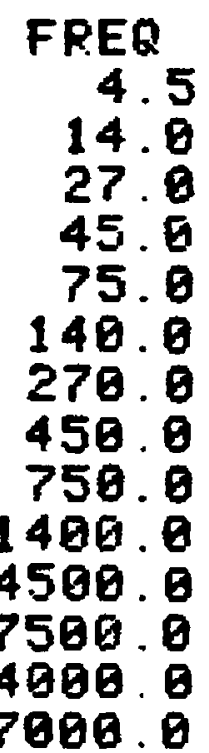 & $\begin{array}{r}\text { AP-RES } \\
33.74 \\
48.79 \\
25.49 \\
26.43 \\
37.55 \\
55.79 \\
42.84 \\
94.85 \\
85.33 \\
159.71 \\
104.71 \\
375.29 \\
437.11 \\
29.93\end{array}$ & $\begin{array}{c}\text { N OBS } \\
1 \\
4 \\
5 \\
7 \\
16 \\
9 \\
7 \\
8 \\
7 \\
4 \\
7 \\
6 \\
7 \\
1\end{array}$ & $\begin{array}{r}\text { STD ERR } \\
0.00 \\
2.02 \\
1.00 \\
1.78 \\
3.13 \\
3.33 \\
6.33 \\
3.73 \\
13.58 \\
25.67 \\
15.23 \\
5.35 \\
10.78 \\
0.08\end{array}$ \\
\hline
\end{tabular}

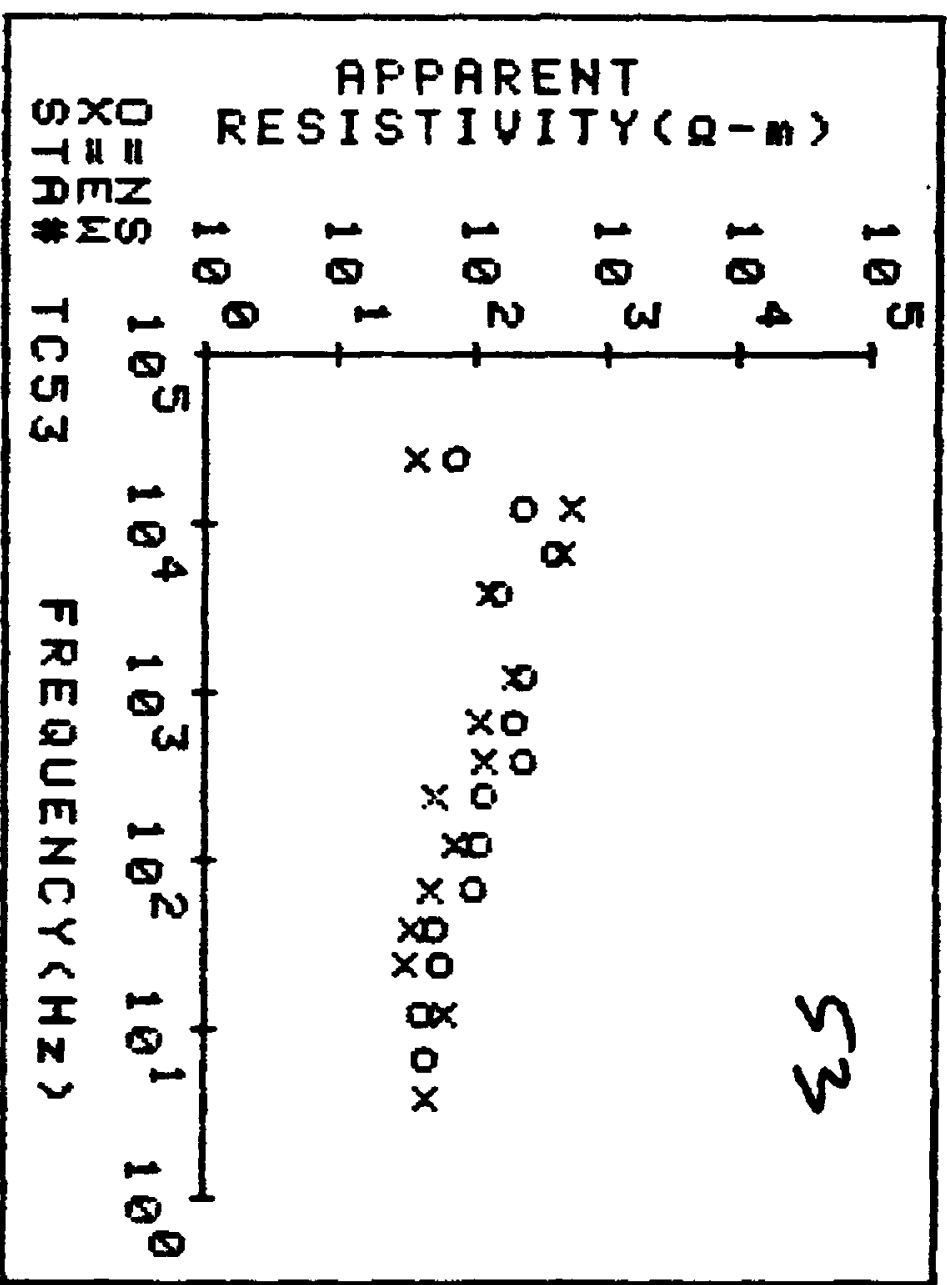

STA-IDTC54FILE NAME $=$ TC54

P̄ROJEC̄T=JĀCKSONON HOLE

STA. ID_TC54 NS NO FREQ $=14$

FREQ AP-RES N OBE STD EFR $\begin{array}{lllll}4.5 & 78.77 & 4 & 10.91\end{array}$

$\begin{array}{llll}7.5 & 34.62 & 6 & 3.60\end{array}$

$\begin{array}{llll}14.0 & 43.88 & 6 & 10.28\end{array}$

$\begin{array}{llll}27.8 & 14.53 & 4 & 2.32\end{array}$

$\begin{array}{llll}45.0 & 25.42 & 5 & 2.36\end{array}$

$\begin{array}{llll}75.0 & 74.64 & ? & 17.11\end{array}$

$\begin{array}{llll}140.8 & 67.09 & 6 & 9.43\end{array}$

$\begin{array}{llll}270.0 & 149.28 & 7 & 9.44\end{array}$

$\begin{array}{llll}450.6 & 571.67 & 5 & 83.64\end{array}$

$\begin{array}{llll}750.0 & 249.67 & 8 & 74.87\end{array}$

$1400.0 \quad 573.61 \quad 1 \quad 0$.

$\begin{array}{llll}4500.0 & 426.44 & 9 & 87.13\end{array}$

$7500.8613 .98 \quad 8 \quad 110.42$

$14800.0654 .54 \quad 3 \quad 12.54$

STA. ID_TC54 EH NO FREQ $=13$

\begin{tabular}{|c|c|c|c|}
\hline $\begin{array}{r}\text { FREQ } \\
4.5 \\
7.5 \\
14.0 \\
27.0 \\
45.0 \\
75.0 \\
140.0 \\
270.0 \\
450.0 \\
4506.0 \\
7506.0\end{array}$ & $\begin{array}{r}\text { AP-RES } \\
12.37 \\
15.29 \\
9.82 \\
5.93 \\
12.30 \\
31.17 \\
36.73 \\
49.56 \\
66.63 \\
133.18 \\
173.56 \\
196.07 \\
98.41\end{array}$ & $\begin{array}{r}\text { N OBS } \\
6 \\
8 \\
16 \\
4 \\
7 \\
10 \\
18 \\
7 \\
5 \\
10 \\
7 \\
4\end{array}$ & $\begin{array}{r}\text { STD E } \\
3 . \\
1 . \\
1 . \\
\\
2 . \\
4 \\
8 . \\
12 \\
72\end{array}$ \\
\hline
\end{tabular}

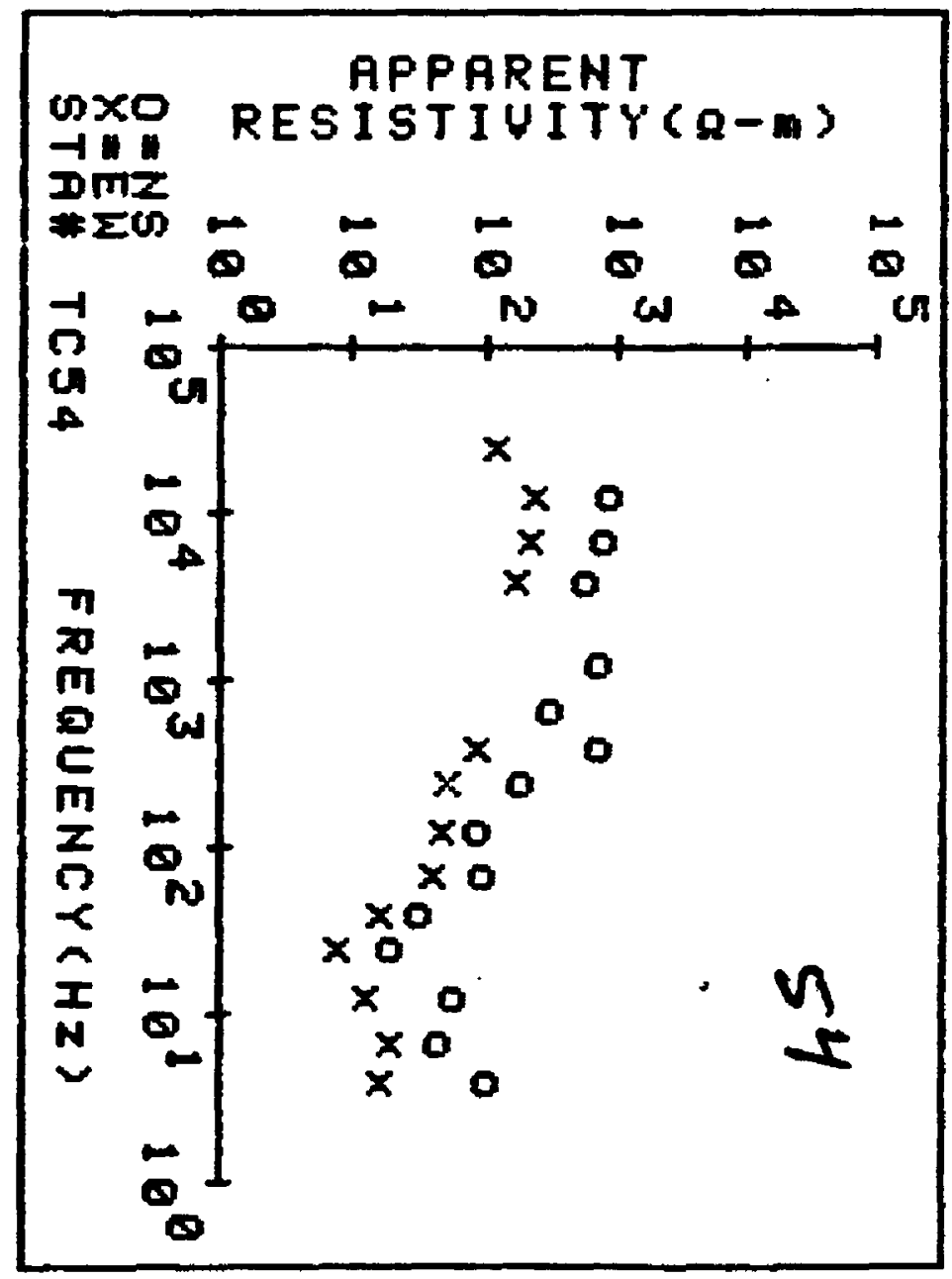


PROJECTT=JĀCKSOON HOLE 94

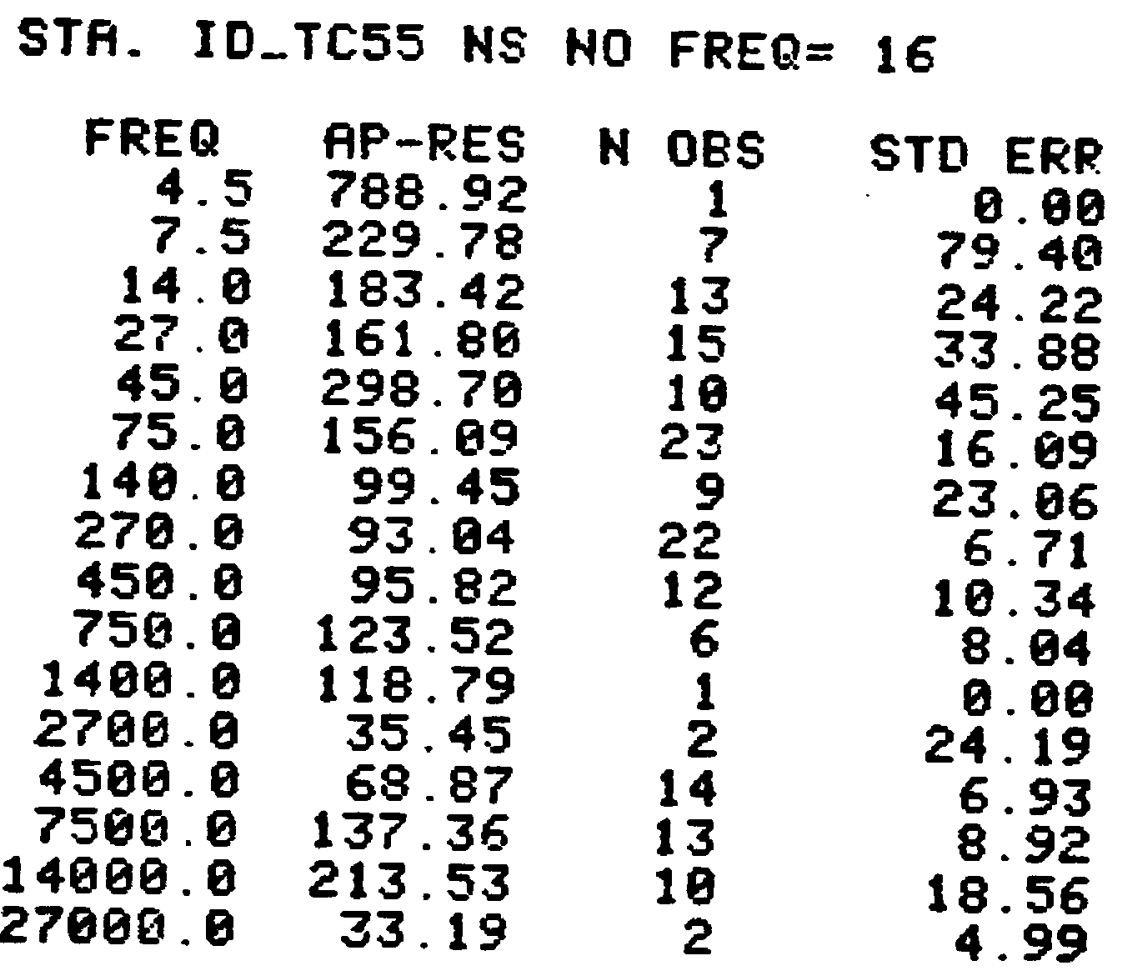

STA. ID_TCSS EW NO FREQ $=16$

$\begin{array}{rrrr}\text { FREQ } & \text { AP-RES } & \text { N OES } & \text { STD ERR } \\ 4.5 & 769.36 & 4 & 139.73 \\ 7.5 & 566.36 & 15 & 83.16 \\ 14.0 & 625.14 & 12 & 191.15 \\ 27.0 & 569.32 & 22 & 39.98 \\ 45.0 & 445.75 & 15 & 44.73 \\ 75.0 & 582.35 & 19 & 71.38 \\ 146.0 & 276.21 & 15 & 24.46 \\ 270.0 & 159.36 & 20 & 9.85 \\ 450.0 & 177.88 & 8 & 19.20 \\ 750.0 & 89.91 & 8 & 17.83 \\ 1409.0 & 93.90 & 5 & 12.51 \\ 2790.9 & 15.57 & 5 & 9.14 \\ 4500.0 & 39.32 & 12 & 6.79 \\ 7590.0 & 142.49 & 12 & 6.98 \\ 4900.0 & 168.64 & 2 & 23.35 \\ 7000.0 & 90.55 & 3 & 10.01\end{array}$

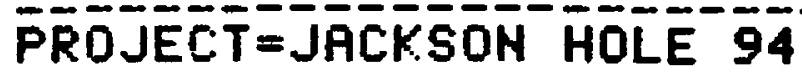

STA. ID_TC56 NS NO FREQ $=16$

$\begin{array}{rrrr}\text { FREQ } & \text { AP-RES } & \text { N ORS } & \text { STO ERR } \\ 4.5 & 570.31 & 4 & 49.35 \\ 7.5 & 316.24 & 19 & 43.24 \\ 14.0 & 366.09 & 9 & 53.35 \\ 27.9 & 267.12 & 7 & 35.65 \\ 45.0 & 184.51 & 10 & 27.07 \\ 75.0 & 142.17 & 9 & 19.33 \\ 140.0 & 89.79 & 9 & 6.39 \\ 270.0 & 51.36 & 9 & 5.85 \\ 450.0 & 97.60 & 10 & 9.55 \\ 759.0 & 113.76 & 11 & 20.77 \\ 1400.0 & 128.23 & 8 & 8.33 \\ 2700.0 & 228.54 & 9 & 29.41 \\ 4500.0 & 169.62 & 12 & 10.80 \\ 7509.0 & 145.38 & 5 & 7.44 \\ 14000.0 & 254.93 & 3 & 1.90 \\ 27000.0 & 182.57 & 3 & 9.90\end{array}$

STA. ID_TC56 EW NO FREQ $=16$

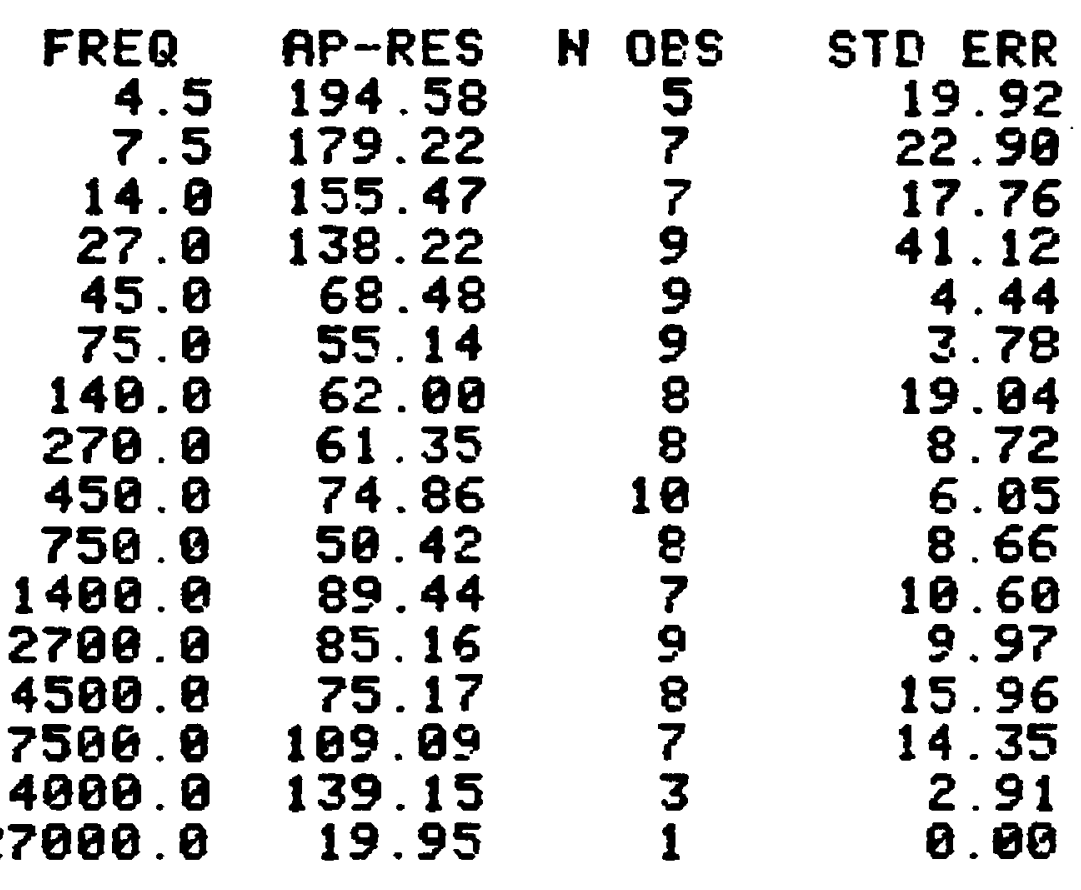

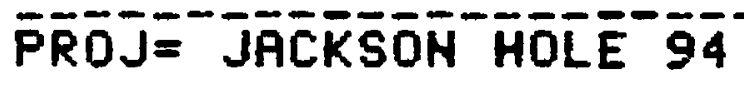
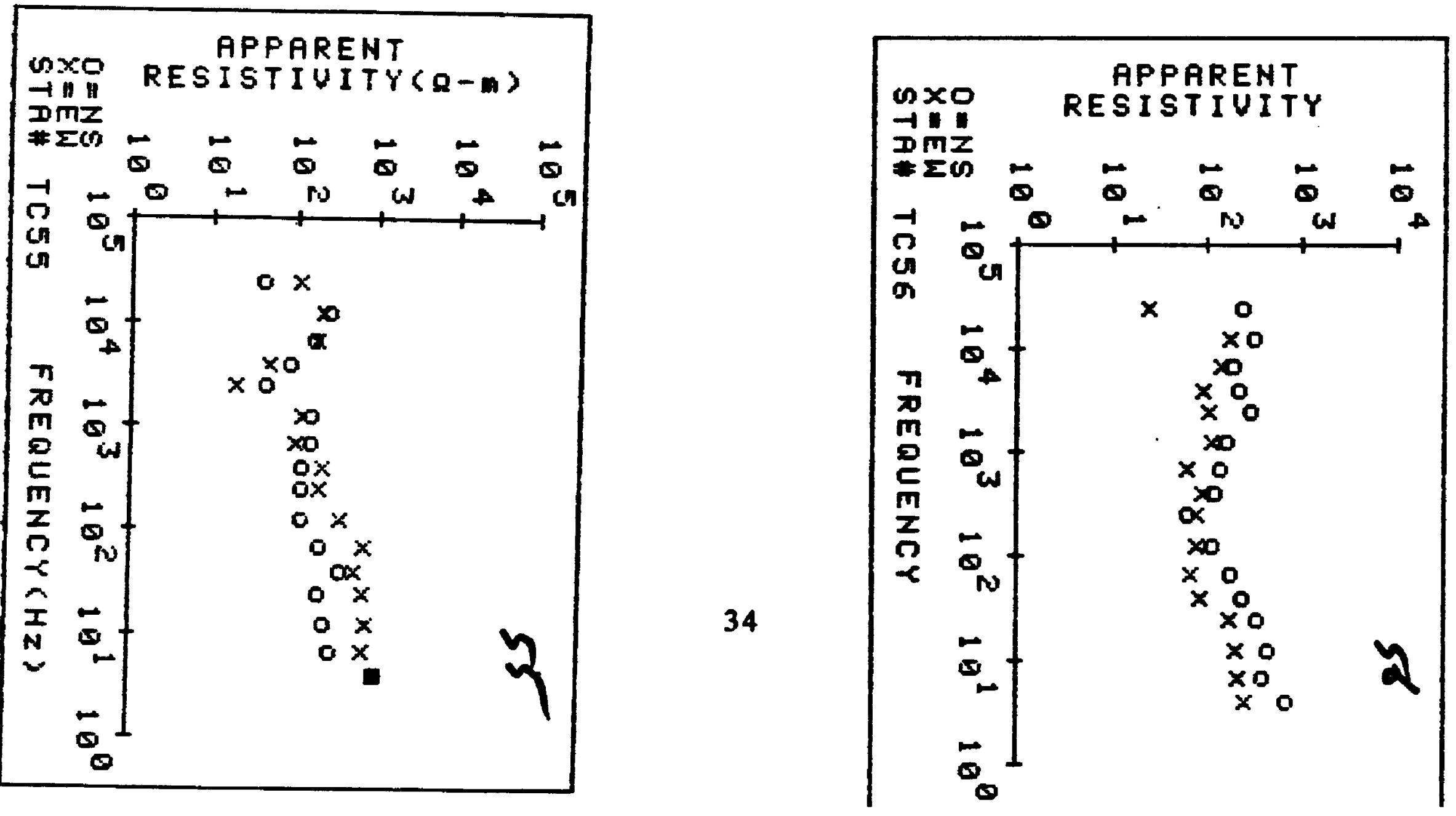
PROJECTT=JACKSON HOLE

STA. ID_TC57 NS NC FREQ = 10

FREQ AF-RES N OBS STD ERR

$7.5766 .00 \quad 7 \quad 29.00$

$14.0 \quad 642.09 \quad 9 \quad 150.00$

$27.0 \quad 559.00 \quad 10 \quad 92.00$

$\begin{array}{llll}45.0 & 644.80 & 11 & 30.09\end{array}$

75.0675 .00

$140.0 \quad 417.00$

$270.0 \quad 313.00$

$450.0 \quad 416.80$

2700.0113 .00

7500.099 .00

9
19

1111.00

112.90

92.20

23.60

STA. ID_TC57 EH NO FREQ $=15$

FREQ AF-RES $N$ OES STD ERR

4.5252 .60

$7.5 \quad 292.00$

14. 379.00

$27.0 \quad 929.00$

$45.0 \quad 1394.00$

75.91722 .00

140.0997 .00

270.0937 .00

$450.0 \quad 654.00$

$750.0 \quad 229.06$

2700.0149 .00

4500.0334 .09

7500.0

159.00

14000.0

165.00

45.80

$\begin{array}{rr}5 & 21.00 \\ 9 & 55.00 \\ 12 & 45.00 \\ 9 & 111.00 \\ 13 & 179.00 \\ 13 & 178.00 \\ 9 & 119.00 \\ 6 & 36.00 \\ 10 & 72.00 \\ 9 & 24.00 \\ 9 & 11.00 \\ 9 & 46.00 \\ 9 & 12.00 \\ 3 & 12.00 \\ 2 & 12.00\end{array}$

PROJJECT $=\overline{J A C K} \bar{S} \bar{O} \overline{\mathrm{N}}$ HOLE $\overline{9} \overline{4}$

STA. ID_TC58 NS NO FREQ $=15$

FREQ AP-RES N OES STD ERR $\begin{array}{llll}4.5 & 903.99 & 3 & 243.96\end{array}$

$\begin{array}{llll}7.5 & 575.58 & 7 & 95.36\end{array}$

$\begin{array}{llll}14.8 & 352.35 & 6 & 74.47\end{array}$

$\begin{array}{llll}27.0 & 486.11 & 6 & 54.75\end{array}$

$\begin{array}{lllll}45.0 & 415.11 & 8 & 27.35\end{array}$

$\begin{array}{llll}75.0 & 315.87 & 9 & 31.80\end{array}$

$140.0 \quad 169.13$

$270.0 \quad 73.60$

$450.6 \quad 76.27$

5.52

$750.0 \quad 78.92$

$2700.0 \quad 129.70$

$4590.0 \quad 344.07$

$7500.0 \quad 193.08$

$14800.0 \quad 484.94$

27000.01071 .00

3.86

2.65

10.00

114.60

3.70

73.83

0.00

STA. ID_TC58 EH NO FPEQ $=15$

FREQ AP-RES N OPS STD ERR

$4.5 \quad 100.04 \quad 3 \quad 4.25$

$\begin{array}{llll}7.5 & 43.52 & 8 & 10.39\end{array}$

$\begin{array}{llll}14.6 & 42.94 & 8 & 7.39\end{array}$

$\begin{array}{llll}27.0 & 60.09 & 18 & 16.98\end{array}$

$\begin{array}{llll}45.6 & 166.48 & 8 & 17.37\end{array}$

$\begin{array}{llll}75.0 & 71.33 & 8 & 18.93\end{array}$

$140.0 \quad 25.02 \quad 8 \quad 3.60$

$\begin{array}{llll}270.8 & 38.33 & 8 & 7.51\end{array}$

$\begin{array}{lllll}450.0 & 34.93 & 7 & 4.82\end{array}$

$\begin{array}{llll}750.0 & 17.11 & 8 & 3.45\end{array}$

$\begin{array}{lllll}2700.6 & 18.82 & 8 & 3.38\end{array}$

$\begin{array}{llll}4500.0 & 57.33 & 3 & 8.94\end{array}$

$\begin{array}{llll}7500.0 & 117.48 & 8 & 9.56\end{array}$

$14000.0 \quad 117.03 \quad 3 \quad 6.06$

$27800.0 \quad 31.01 \quad 110.00$

P'ROJJ JA'CKSON HOLE $9 \overline{4}$
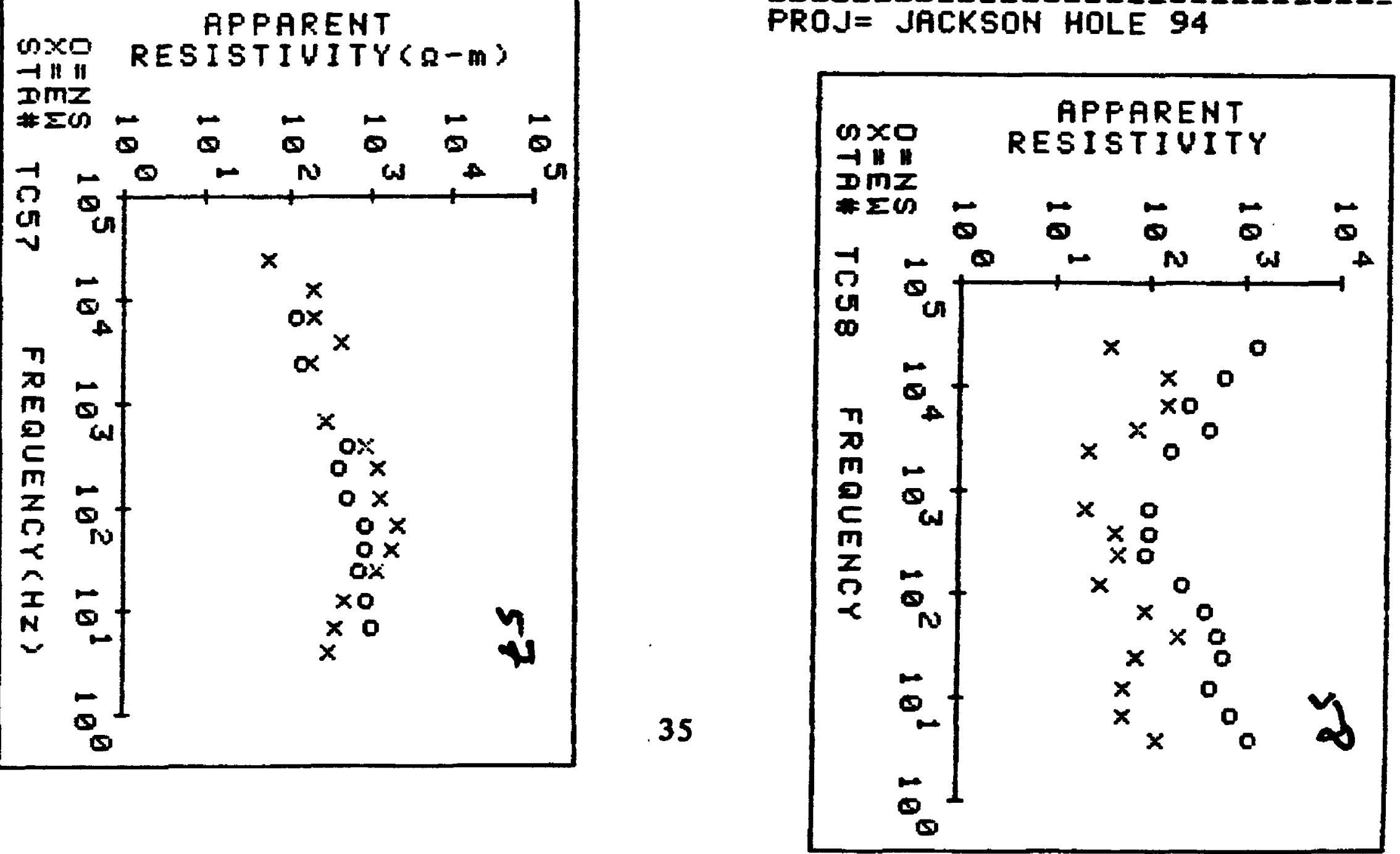
STA. ID_TC59 NS NO FREQ $=16$

\begin{tabular}{|c|c|c|c|}
\hline $\begin{array}{r}\text { FREQ } \\
4.5 \\
7.5 \\
14.0 \\
27.0 \\
45.0 \\
75.0 \\
149.0 \\
279.0 \\
456.0 \\
750.0 \\
490.0 \\
700.0 \\
500.0 \\
500.0 \\
900.0 \\
999.0\end{array}$ & $\begin{array}{r}\text { AP-RES } \\
19.70 \\
12.21 \\
68.96 \\
129.97 \\
124.04 \\
451.11 \\
444.71 \\
519.98 \\
689.79 \\
489.63 \\
237.21 \\
177.97 \\
67.28 \\
61.32 \\
144.23 \\
49.99\end{array}$ & $\begin{array}{c}\text { OES } \\
3 \\
4 \\
6 \\
9 \\
8 \\
8 \\
8 \\
19 \\
10 \\
19 \\
5 \\
7 \\
6 \\
6 \\
3 \\
1\end{array}$ & $\begin{array}{r}\text { STD ERR } \\
2.18 \\
1.52 \\
15.51 \\
10.53 \\
36.03 \\
24.74 \\
97.44 \\
75.96 \\
34.75 \\
69.36 \\
42.16 \\
15.37 \\
5.90 \\
1.62 \\
11.52 \\
0.00\end{array}$ \\
\hline
\end{tabular}

STA. ID_TC59 EW NO FREQ $=16$

\begin{tabular}{|c|c|c|c|}
\hline $\begin{array}{r}\text { FREQ } \\
4.5 \\
7.5 \\
14.0 \\
27.0 \\
45.0 \\
75.0 \\
140.0 \\
270.0 \\
450.0 \\
750.8 \\
490.0 \\
760.0 \\
1500.0 \\
560.0 \\
1000.0\end{array}$ & $\begin{array}{r}\text { AF-RES } \\
42.43 \\
112.17 \\
75.14 \\
182.34 \\
122.49 \\
298.72 \\
188.31 \\
276.48 \\
380.05 \\
279.55 \\
210.97 \\
19.93 \\
108.93 \\
126.31 \\
278.79 \\
18.88\end{array}$ & $\begin{array}{c}\text { N OBS } \\
3 \\
5 \\
6 \\
7 \\
8 \\
9 \\
10 \\
10 \\
11 \\
11 \\
6 \\
4 \\
8 \\
6 \\
3 \\
1\end{array}$ & $\begin{array}{r}\text { STD ERR } \\
2.35 \\
2.42 \\
4.34 \\
5.64 \\
8.63 \\
16.58 \\
12.56 \\
15.06 \\
10.85 \\
20.55 \\
24.22 \\
5.32 \\
10.94 \\
4.59 \\
2.62 \\
9.99\end{array}$ \\
\hline
\end{tabular}

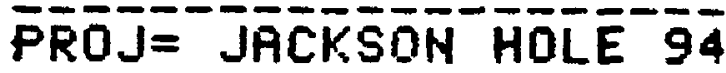

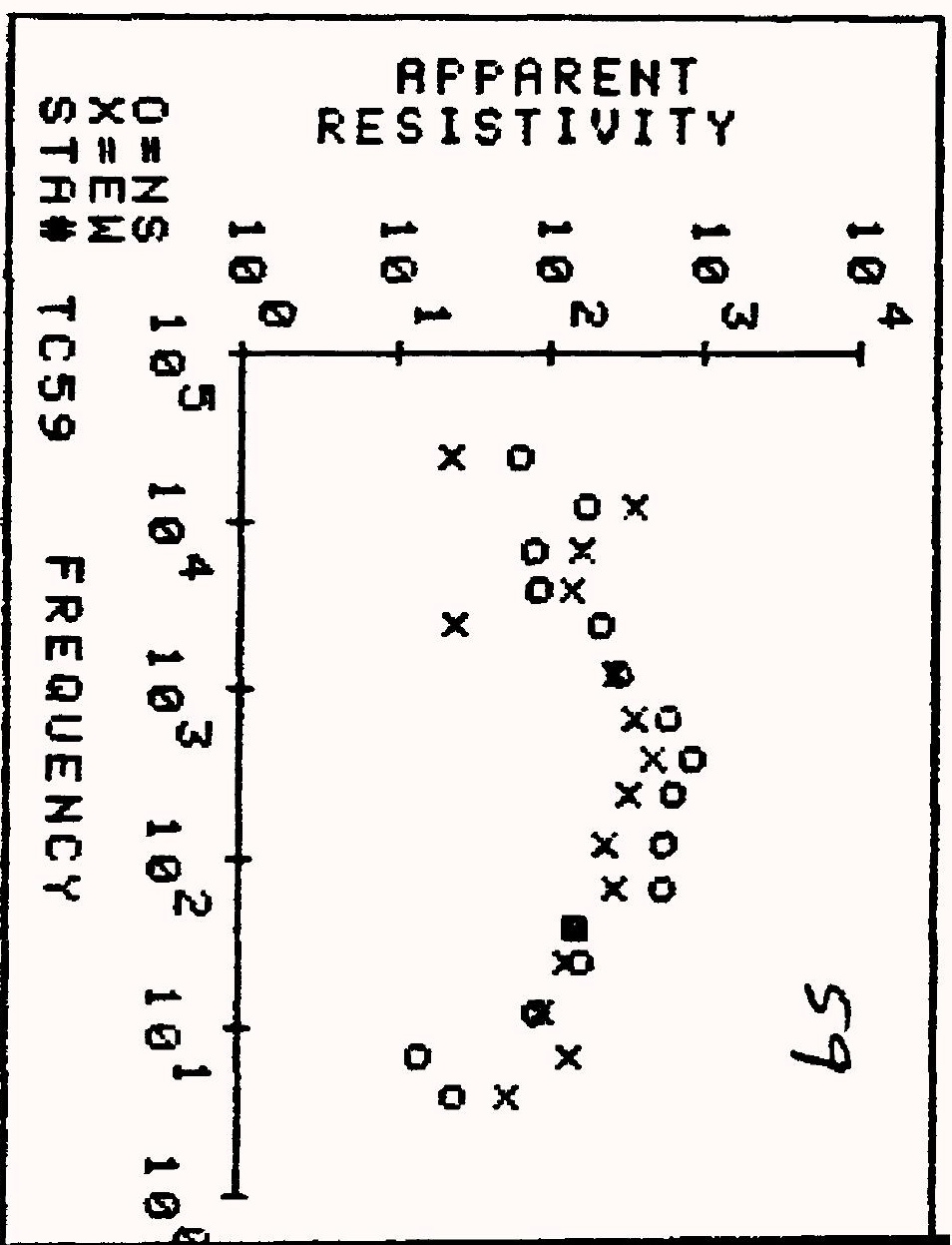

PROJ $=$ JACKSON HOLE 94

STR-IDTC6EF ILE NAME $=$ TC60

P̄ROJECT=JĀCKSOON HOLEE $9 \overline{9}$

STA. ID_TC60 NS NO FREQ $=12$

FREQ AP-RES N OBS STD ERR $\begin{array}{llll}7.5 & 42.93 & 9 & 19.56\end{array}$

$\begin{array}{llll}14.0 & 111.48 & 7 & 149.56\end{array}$

$27.8 \quad 136.53 \quad 17 \quad 32.45$

$45.8 \quad 83.60 \quad 1 \quad 9.60$

$148.0 \quad 219.62 \quad 29 \quad 23.33$

$\begin{array}{llll}450.0 & 382.37 & 13 & 32.02\end{array}$

$\begin{array}{llll}758.8 & 294.34 & 8 & 30.37\end{array}$

$2700.0 \quad 441.91 \quad 3 \quad 137.16$

$\begin{array}{llll}4500.0 & 273.01 & 21 & 11.37\end{array}$

$7580.0 \quad 240.94 \quad 20 \quad 9.53$

$14000.0 \quad 398.85 \quad 3 \quad 101.86$

$\begin{array}{llll}27000.0 & 210.54 & 1 & 0.00\end{array}$

STA. ID_TC6O EH NO FREQ $=13$

FREQ AP-RES N OES STD ERR $\begin{array}{llll}4.5 & 117.83 & 4 & 81.52\end{array}$

$\begin{array}{llll}7.5 & 61.31 & 15 & 6.69\end{array}$

$\begin{array}{llll}14.9 & 86.46 & 16 & 9.38\end{array}$

$27.8158 .66 \quad 21 \quad 5.35$

$\begin{array}{llll}140.6 & 427.03 & 13 & 24.82\end{array}$

$278.0 \quad 417.91 \quad 21 \quad 21.41$

$\begin{array}{llll}450.0 & 524.48 & 13 & 10.48\end{array}$

$758.6 \quad 354.17 \quad 13 \quad 22.84$

$1460.6 \quad 375.96 \quad 5 \quad 63.17$

$2700.0 \quad 223.50 \quad 6 \quad 48.26$

$4500.0 \quad 397.72 \quad 23 \quad 13.65$

$7560.0 \quad 379.93 \quad 12 \quad 24.29$

$\begin{array}{llll}14000.0 & 295.34 & 2 & 77.39\end{array}$

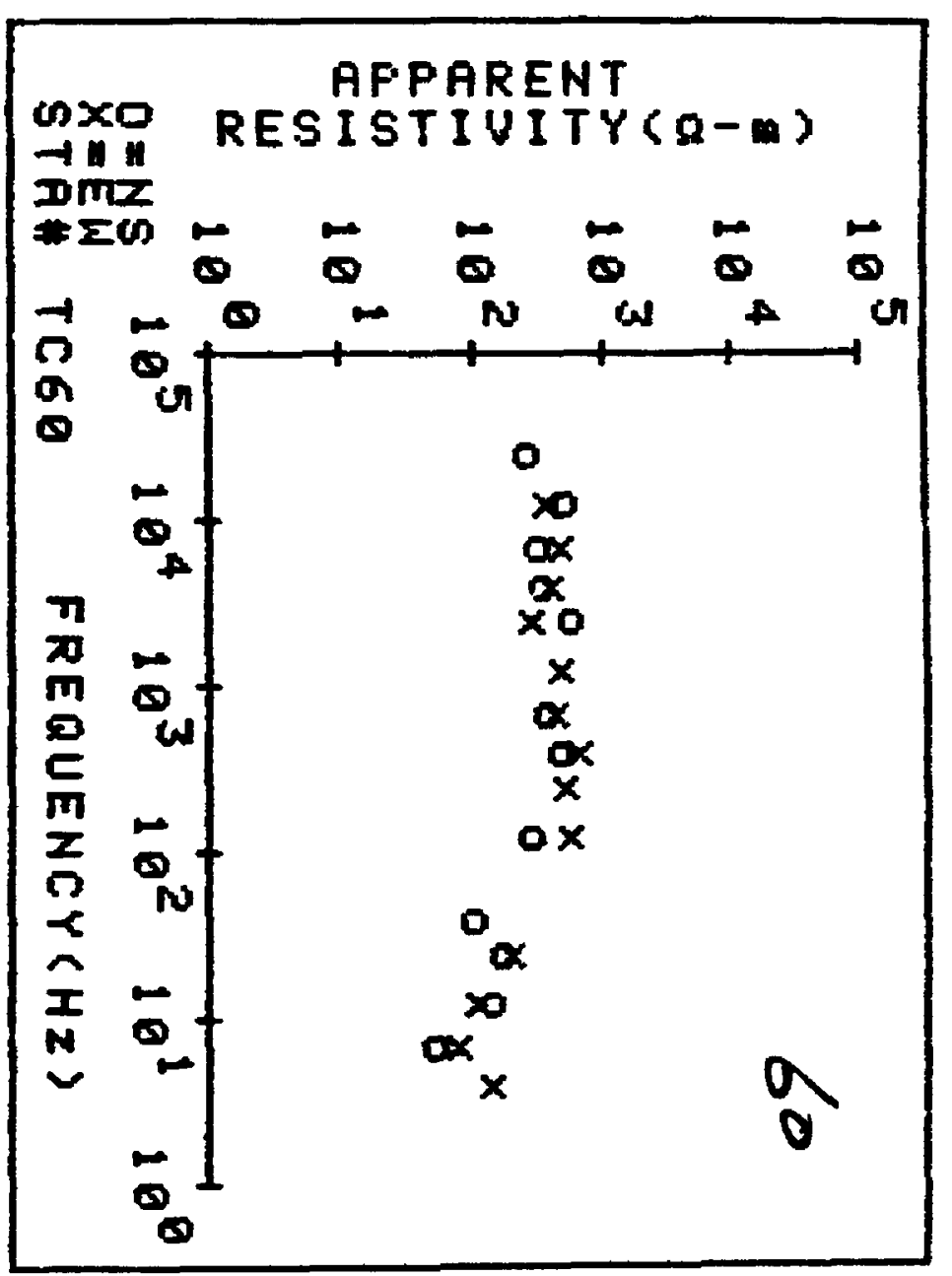


STA. ID_TC61 NS NO FREQ $=15$

$\begin{array}{rrrr}\text { FREQ } & \text { AP-RES } & N \text { OBS } & \text { STO ERR } \\ 4.5 & 478.65 & 4 & 10.11 \\ 7.5 & 58.19 & 3 & 2.85 \\ 14.0 & 255.55 & 3 & 131.50 \\ 27.0 & 869.13 & 3 & 34.18 \\ 45.0 & 1770.56 & 4 & 83.88 \\ 75.0 & 725.96 & 6 & 273.31 \\ 140.0 & 828.06 & 7 & 153.41 \\ 270.0 & 593.98 & 8 & 86.70 \\ 450.0 & 825.93 & 6 & 18.41 \\ 750.0 & 676.76 & 6 & 138.55 \\ 700.0 & 89.46 & 6 & 6.73 \\ 509.0 & 539.45 & 11 & 25.36 \\ 569.0 & 587.43 & 8 & 39.79 \\ 000.0 & 419.18 & 3 & 23.97 \\ 000.0 & 28.19 & 1 & 0.00\end{array}$

STA. ID_TC61 EH NO FREQ $=16$

$\begin{array}{rrrr}\text { FREQ } & \text { AP-RES } & \text { N OBS } & \text { STD ERR } \\ 4.5 & 69.08 & 3 & 4.69 \\ 7.5 & 132.93 & 4 & 3.92 \\ 7.5 & 66.34 & 7 & 1.64 \\ 14.6 & 142.06 & 6 & 9.72 \\ 27.6 & 268.86 & 7 & 5.83 \\ 45.6 & 335.52 & 9 & 8.54 \\ 75.0 & 418.69 & 8 & 95.18 \\ 149.0 & 416.82 & 9 & 65.87 \\ 270.0 & 427.11 & 19 & 50.75 \\ 456.0 & 549.38 & 8 & 53.34 \\ 750.0 & 253.70 & 8 & 60.97 \\ 706.0 & 98.52 & 3 & 26.77 \\ 500.0 & 264.16 & 8 & 81.37 \\ 509.0 & 668.56 & 8 & 31.90 \\ 090.0 & 936.15 & 3 & 52.65 \\ 000.0 & 24.84 & 1 & 6.09\end{array}$

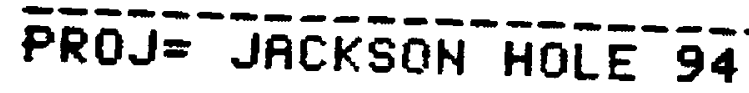

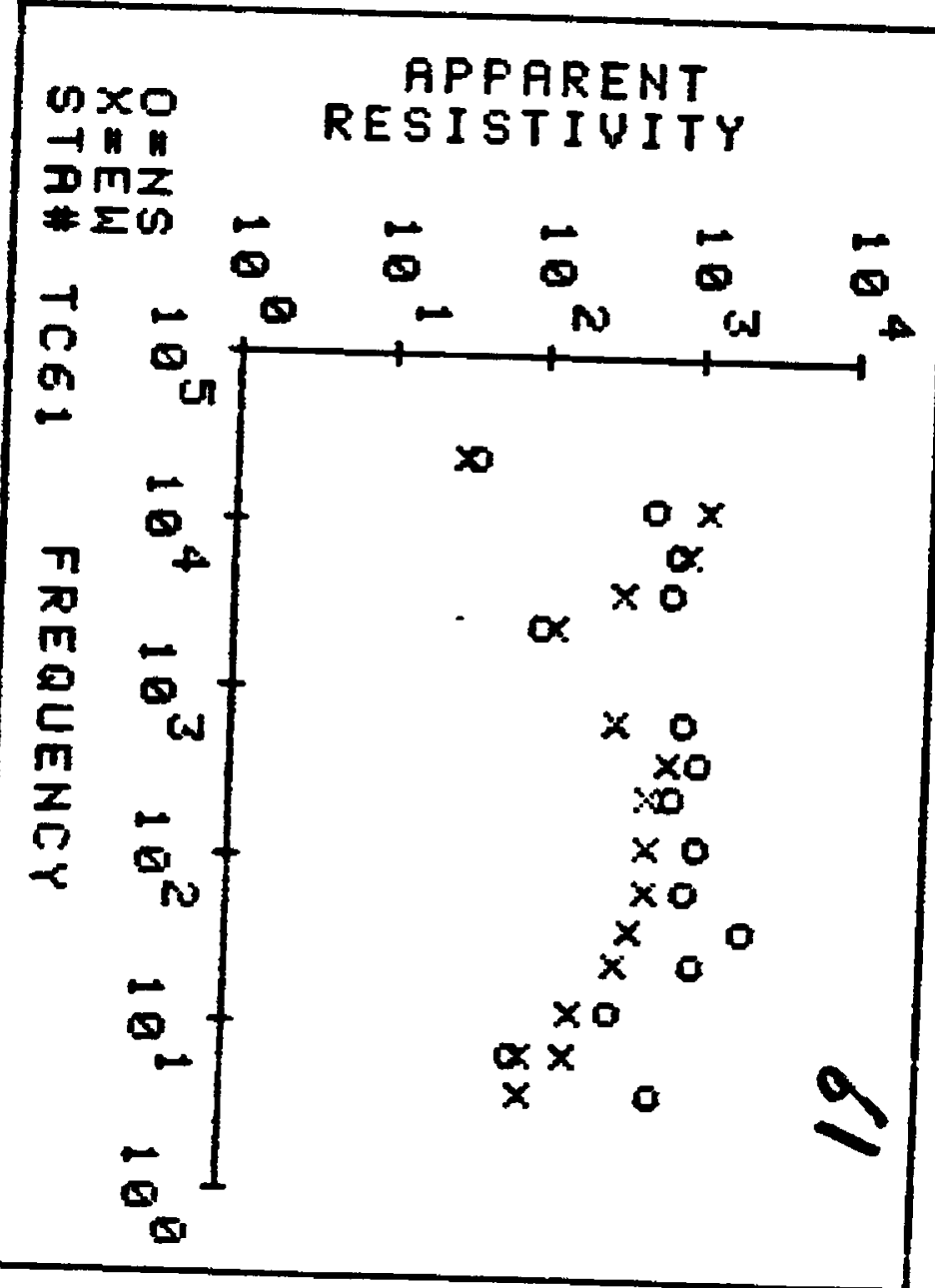

PRŌJEC̄T=JĀKSON HOLE 94

STA. ID_TC.62 NS NO FREQ $=12$

$$
4.5 \quad 65.15
$$

$7.5 \quad 184.13$

N OES STO ERR

$14.6 \quad 65.82$

$27.0 \quad 97.53$

$75.0 \quad 221.62$

$140.6 \quad 128.31$

$270.0 \quad 252.21$

$750.0 \quad 139.30$

$1496.0 \quad 132.72$

4500.0168 .28

$7500.0 \quad 310.13$

$14800.0 \quad 191.91$

$\begin{array}{rr}\text { OES } & \text { STO ERR } \\ 3 & 2.27 \\ 3 & 3.88 \\ 8 & 3.07 \\ 8 & 9.16 \\ 7 & 84.42 \\ 7 & 9.15 \\ 9 & 47.13 \\ 9 & 21.64 \\ 2 & 17.90 \\ 7 & 34.35 \\ 8 & 38.49 \\ 6 & 21.82\end{array}$

STA. ID_TCG2 EN NO FREQ $=12$

$\begin{array}{rrrr}\text { FRER } & \text { AP-RES } & \text { N OBS } & \text { STD ERR } \\ 4.5 & 195.44 & 2 & 8.74 \\ 7.5 & 182.59 & 3 & 8.76 \\ 14.0 & 155.39 & 8 & 15.01 \\ 27.0 & 222.45 & 8 & 30.12 \\ 45.0 & 326.81 & 4 & 38.31 \\ 75.0 & 280.45 & 7 & 37.62 \\ 140.0 & 368.17 & 8 & 36.92 \\ 279.0 & 388.74 & 8 & 46.95 \\ 750.0 & 348.28 & 8 & 62.76 \\ 2700.0 & 594.83 & 2 & 168.22 \\ 7590.0 & 953.58 & 4 & 50.41 \\ 4009.0 & 1211.60 & 3 & 79.88\end{array}$

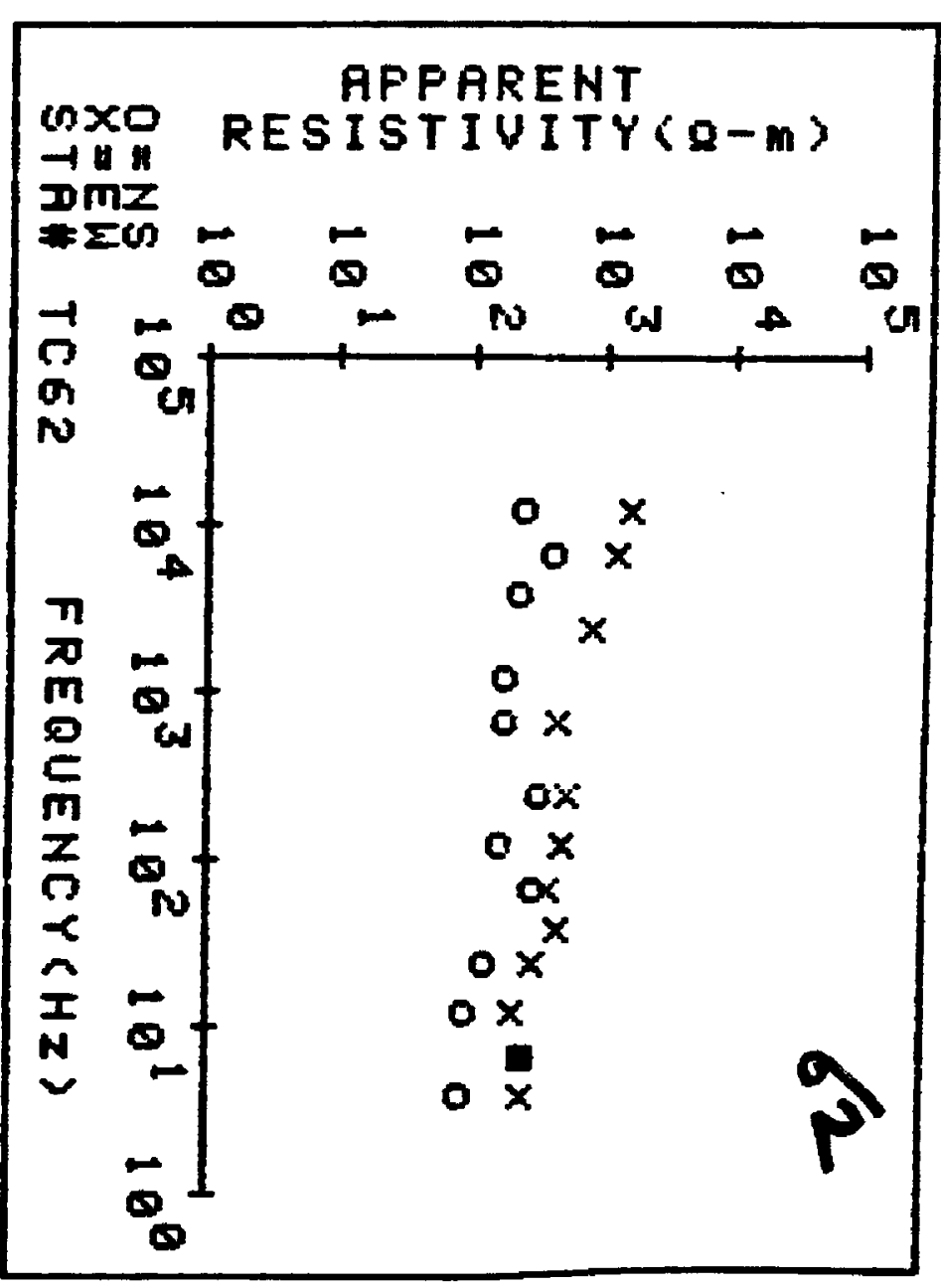


F'ROJECTEJACKSOAN HOLE 94

STA. ID_TC6.3 NS NO FREQ $=13$

$\begin{array}{rrrr}\text { FREQ } & \text { AP-RES } & \text { N OBS } & \text { STD ERR } \\ 4.5 & 320.52 & 3 & 48.49 \\ 14.0 & 163.21 & 6 & 54.16 \\ 45.0 & 99.37 & 4 & 58.19 \\ 75.0 & 67.35 & 5 & 27.72 \\ 140.0 & 193.63 & 6 & 17.38 \\ 270.9 & 129.20 & 9 & 13.22 \\ 450.0 & 285.91 & 8 & 19.32 \\ 750.0 & 283.90 & 8 & 77.88 \\ 460.0 & 416.40 & 7 & 41.77 \\ 2709.0 & 261.56 & 8 & 39.51 \\ 4569.0 & 428.73 & 6 & 23.68 \\ 7509.0 & 485.41 & 6 & 32.86 \\ 4060.0 & 272.50 & 3 & 29.55\end{array}$

5TA. ID_TE63 EW HO FREQ $=15$

FREQ AP-RES $N$ OBS STD ERR

$\begin{array}{rrrr}4.5 & 121.97 & 4 & 5.34 \\ 14.0 & 125.95 & 4 & 1.81 \\ 27.0 & 121.83 & 5 & 144.25 \\ 45.0 & 190.35 & 4 & 10.63 \\ 75.0 & 76.83 & 6 & 1.20 \\ 40.0 & 129.37 & 8 & 48.73 \\ 70.0 & 136.13 & 8 & 16.09 \\ 50.9 & 197.53 & 8 & 10.57 \\ 50.0 & 180.19 & 7 & 33.25 \\ 50.0 & 215.56 & 8 & 280.39 \\ 90.0 & 493.89 & 7 & 39.69 \\ 90.0 & 244.94 & 8 & 21.65 \\ 00.0 & 351.33 & 6 & 6.41 \\ 90.0 & 388.21 & 3 & 127.70 \\ 00.0 & 413.99 & 3 & 16.23\end{array}$

$14000.0 \quad 413.99$
5TA. ID_TC64 NS NO FREQ $=18$

$\begin{array}{rrrr}\text { FREQ } & \text { AP-RES } & \text { N OBS } & \text { STD ERR } \\ 4.5 & 226.39 & 3 & 4.29 \\ 7.5 & 274.66 & 3 & 152.74 \\ 14.0 & 1354.40 & 4 & 262.59 \\ 14.0 & 247.15 & 4 & 3.27 \\ 27.0 & 1813.70 & 5 & 524.51 \\ 45.9 & 2263.90 & 9 & 810.93 \\ 75.0 & 691.66 & 7 & 135.71 \\ 75.0 & 247.06 & 6 & 27.12 \\ 149.0 & 253.02 & 9 & 86.50 \\ 270.0 & 115.27 & 7 & 7.76 \\ 450.0 & 123.58 & 9 & 15.68 \\ 750.0 & 226.53 & 9 & 71.94 \\ 1400.0 & 298.67 & 1 & 0.00 \\ 2700.0 & 276.34 & 10 & 33.22 \\ 4509.0 & 336.15 & 8 & 51.52 \\ 7509.0 & 659.19 & 10 & 71.54 \\ 4909.0 & 673.65 & 3 & 20.79 \\ 7009.0 & 78.47 & 6 & 8.63\end{array}$

5TA. ID_TC64 EH NO FREQ $=15$

FREQ AP-RES N OBS STD ERR

75131.11 N 5170

$14.0 \quad 294.49 \quad 5 \quad 106.51$

$27.61673 .30 \quad 5 \quad 670.67$

$\begin{array}{llll}45.6 & 212.06 & 6 & 34.47\end{array}$

$\begin{array}{llll}75.0 & 221.26 & 8 & 28.33\end{array}$

$\begin{array}{llll}149.0 & 85.37 & 9 & 8.95\end{array}$

$\begin{array}{llll}278.0 & 185.59 & 10 & 11.48\end{array}$

$\begin{array}{llll}450.0 & 87.55 & 9 & 12.51\end{array}$

$\begin{array}{llll}750.0 & 98.68 & 10 & 12.41\end{array}$

$1400.6 .639 .41 \quad 4 \quad 43.83$

$\begin{array}{llll}2700.0 & 79.49 & 9 & 60.75\end{array}$

$\begin{array}{llll}4500.6 & 71.71 & 7 & 11.36\end{array}$

$\begin{array}{llll}7500.0 & 135.45 & 8 & 7.98\end{array}$

$14000.6 \quad 44.25 \quad 3 \quad 6.16$

$\begin{array}{llll}27000.0 & 4.28 & 3 & .26\end{array}$
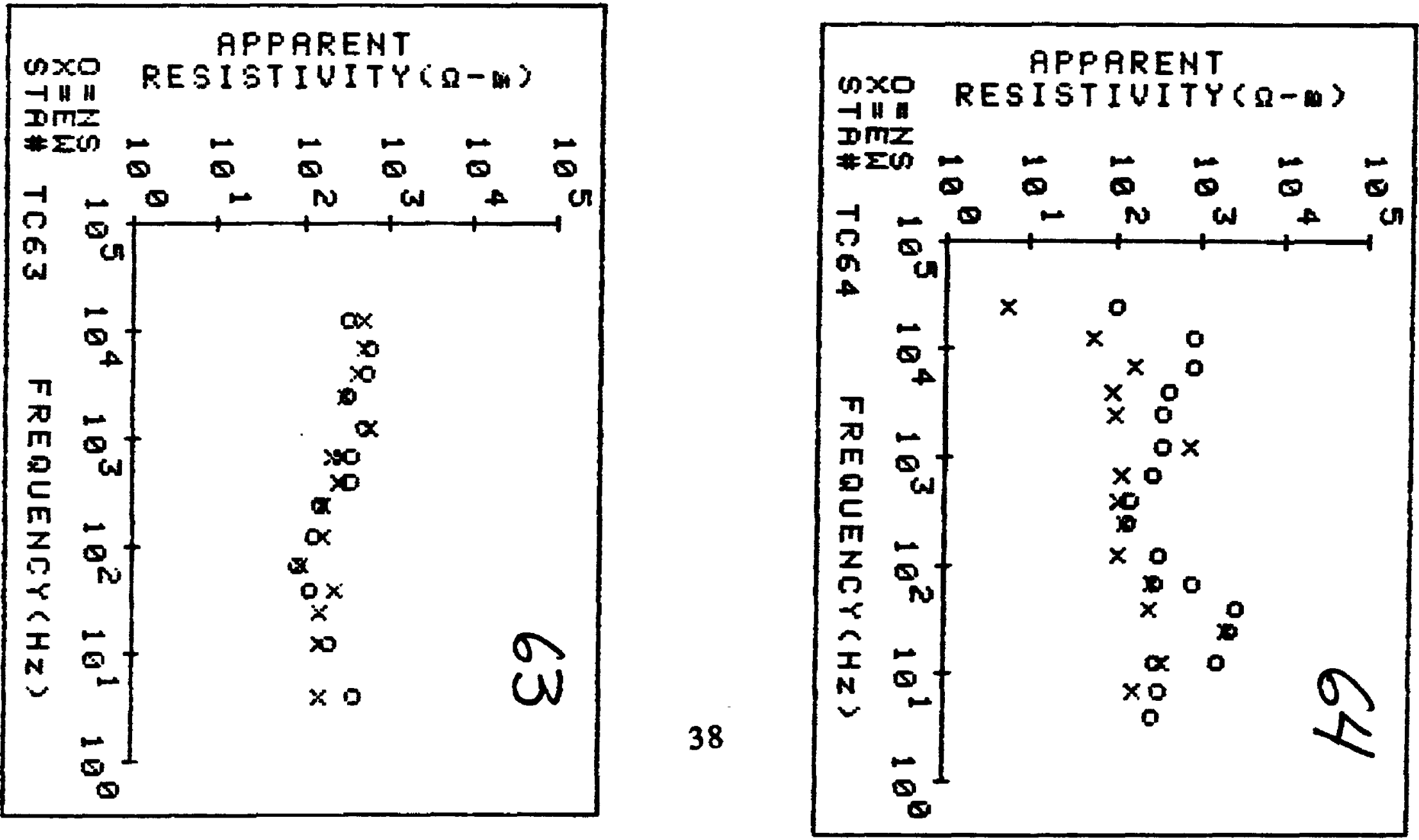


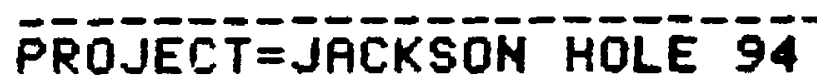

$\begin{array}{rrcr}\text { STA. ID_TC65 NS NO FREQ }=10 \\ \text { FREQ } & \text { AP-RES } & \text { N OBS } & \text { STD ERR } \\ 7.5 & 395.66 & 4 & 1.77 \\ 14.0 & 264.91 & 5 & 12.05 \\ 27.0 & 128.57 & 4 & 4.31 \\ 75.0 & 43.71 & 4 & 17.40 \\ 140.0 & 54.57 & 4 & .94 \\ 270.6 & 64.14 & 4 & 2.77 \\ 450.0 & 32.35 & 5 & 4.71 \\ 2700.0 & 71.92 & 4 & 4.33 \\ 4500.0 & 270.33 & 4 & 36.21 \\ 14009.0 & 141.97 & 3 & 8.22\end{array}$

STA. ID_TC6.5 EH NO FREQ $=8$

FREQ AP-RES $N$ OBS STD ERR.

$7.5 \quad 146.96$

14.6272 .03

$27.0 \quad 353.56$

$45.0 \quad 88.52$

$140.6 \quad 29.45$

$750.0 \quad 267.02$

2700.0104 .68

27069.037 .15

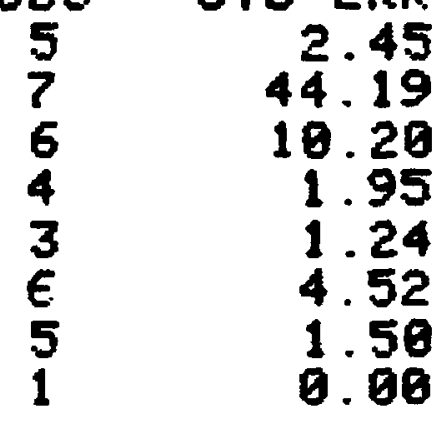

APPARENT

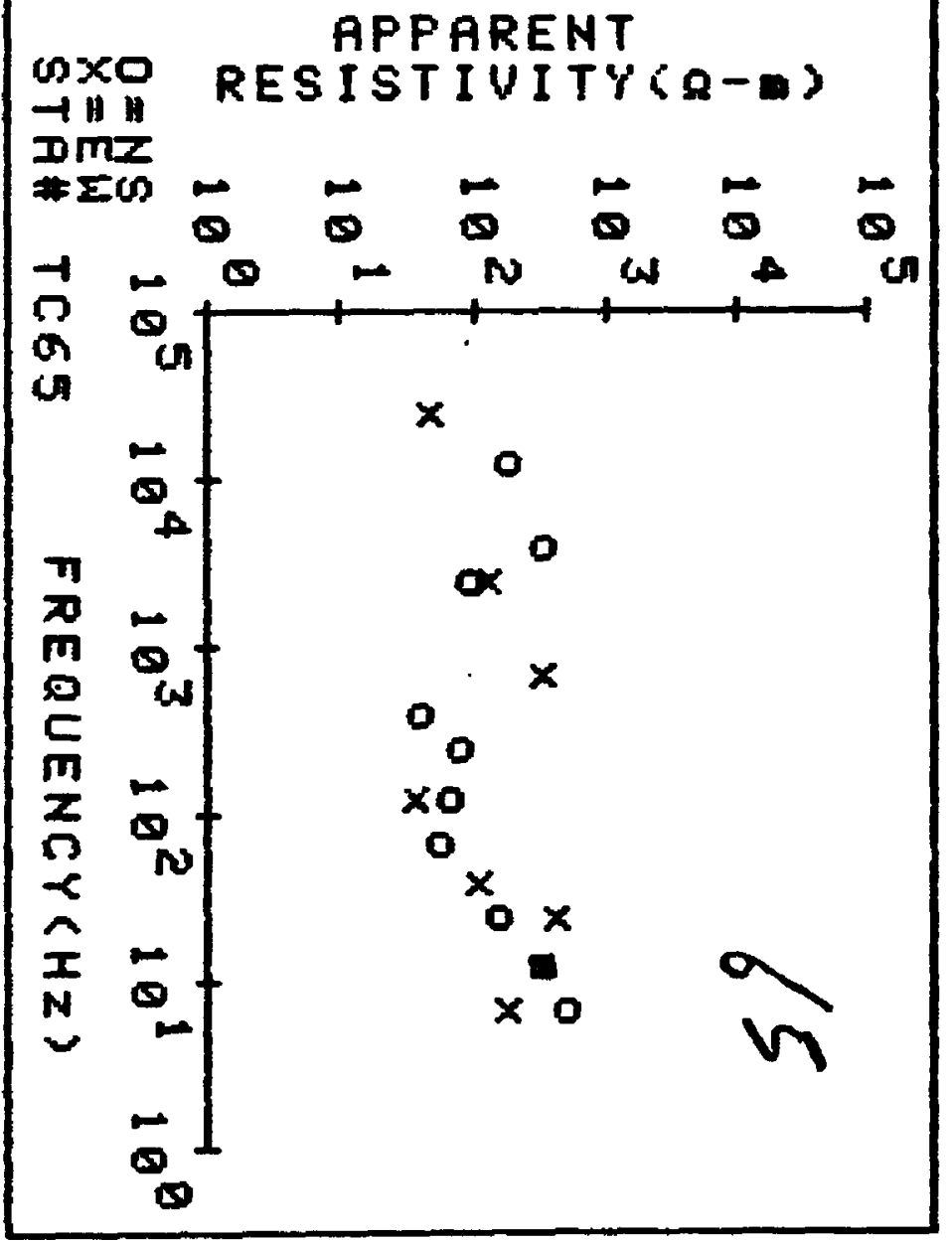

PROOJJE T́T

STA. ID_TC66 NS NO FREQ $=16$

FREQ AP-RES $N$ ORE STD ERR $4.5 \quad 2.10$

$7.5 \quad 28.40$

$14.8 \quad 25.51$

$27.8 \quad 10.93$

45.016 .73

$75.0 \quad 22.42$

140.020 .27

$270.0 \quad 23.19$

450.053 .16

750.0

1460.8

72.87

11.63

2709.0

4500.0

7500.8

14000.0

47.51

15.73

8.55

164.31

57.58

08
5
6
9
7
10
10
10
10
9
3
3
9
4
4
3
1

2.05

4.20

3.45

1.98

1.35

1.04

1.96

4.14

4.50

2.90

3.48

.51

.20

7.51

0.00

STA. ID_TC66 EH NO FREQ $=12$

FREQ AP-RES $N$ OES STD ERR $4.5 \quad 14.05$

$7.5 \quad 28.29$

$\begin{array}{rr}3 & .07 \\ 6 & 3.58 \\ 6 & 3.03 \\ 8 & 23.85 \\ 7 & 49.45 \\ 19 & 8.84 \\ 9 & 12.17 \\ 6 & 6.29 \\ 8 & 11.29 \\ 7 & 9.94 \\ 3 & 1.52 \\ 3 & 2.03\end{array}$

$27.0 \quad 471.56$

$45.8 \quad 228.12$

$75.0 \quad 282.23$

140.0116 .77

$270.0 \quad 82.11$

$456.0 \quad 139.58$

750.0207 .87

14008.0257 .37

27000.0

46.35

2.03

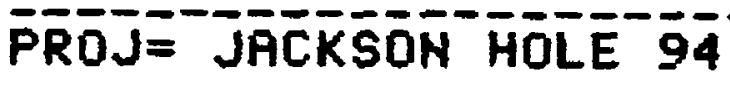

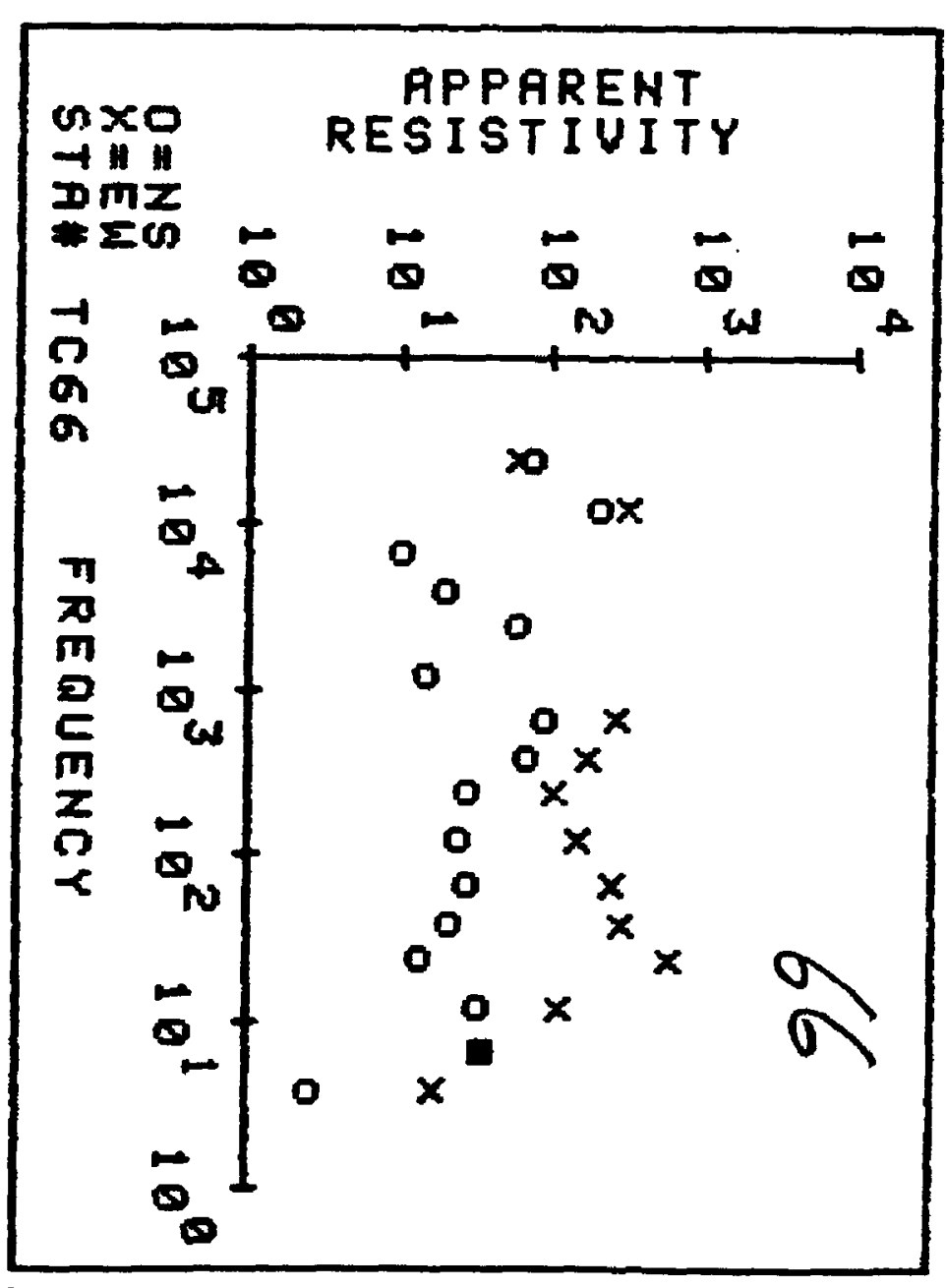


PROJ $=$ JACKSON HOLE 94

STA-IDTC67F ILE NFME=TC67

P̄ROJJECT =JĀKKSON HOLE $9 \overline{94}$

STA. ID_TC67 NS NO FREQ $=11$

$\begin{array}{rrrr}\text { FREQ } & \text { AP-RES } & \text { N OES } & \text { STD ERR } \\ 4.5 & 23.61 & 5 & 1.22 \\ 7.5 & 6.29 & 5 & .52 \\ 14.0 & 16.97 & 7 & 1.10 \\ 45.0 & 14.83 & 8 & 2.13 \\ 75.0 & 14.94 & 19 & 1.92 \\ 149.0 & 15.52 & 9 & 1.55 \\ 270.0 & 24.25 & 9 & 2.78 \\ 450.0 & 20.77 & 7 & 2.55 \\ 750.0 & 45.76 & 9 & 2.72 \\ 4909.0 & 59.09 & 3 & 18.51 \\ 7090.0 & 79.53 & 1 & 0.00\end{array}$

STA. ID_TCE? EW NO FREQ $=12$

FREQ AP-RES N OBS STD ERR

$\begin{array}{rrrr}4.5 & 8.11 & 4 & .40 \\ 7.5 & 6.42 & 6 & .77 \\ 14.0 & 12.46 & 19 & .68 \\ 27.0 & 11.48 & 9 & 1.09 \\ 45.9 & 10.24 & 10 & 1.04 \\ 75.0 & 12.05 & 10 & 1.79 \\ 40.9 & 17.71 & 9 & 1.08 \\ 70.0 & 10.61 & 8 & 1.85 \\ 50.9 & 30.61 & 6 & 3.97 \\ 59.9 & 30.53 & 5 & 2.76 \\ 60.0 & 165.64 & 3 & .36 \\ 90.0 & 133.81 & 3 & 7.89\end{array}$

$27000.0133 .81 \quad 3 \quad 7.89$
PROJECT $=$ JACKSON HOLE 94

STA. ID_TC68 NS NO FREQ $=15$

FREQ AP-RES N OBS STD ERR

\begin{tabular}{|c|c|}
\hline $\begin{array}{r}2.30 \\
13.30 \\
8.61 \\
4.47 \\
7.78 \\
10.90 \\
23.48 \\
23.18 \\
47.81 \\
38.81 \\
20.33 \\
19.33 \\
20.72 \\
109.16 \\
21.49\end{array}$ & $\begin{array}{r}3 \\
3 \\
9 \\
10 \\
10 \\
9 \\
9 \\
8 \\
7 \\
7 \\
3 \\
3 \\
7 \\
3 \\
3\end{array}$ \\
\hline
\end{tabular}

STA. ID_TC68 EW NO FREQ $=13$

FREQ AP-RES N OBS STD ERR

$$
4.5 \quad 4.22
$$

7.5

8.08

14.0

9.26

27.8

9.09

45.0

12.48

75.8

13.23

140.0

21.13

270.0

450 . 0

16.23

35.97

750.0

32.12

5.68

4500 . 0

7500.8

32.49

70.40
3
8
11
10
10
10
10
8
9
9
4
5
3

.59

.42

.65

.71

1.45

1.66

2.14

2.72

4.54

.28

2.19

1. 48

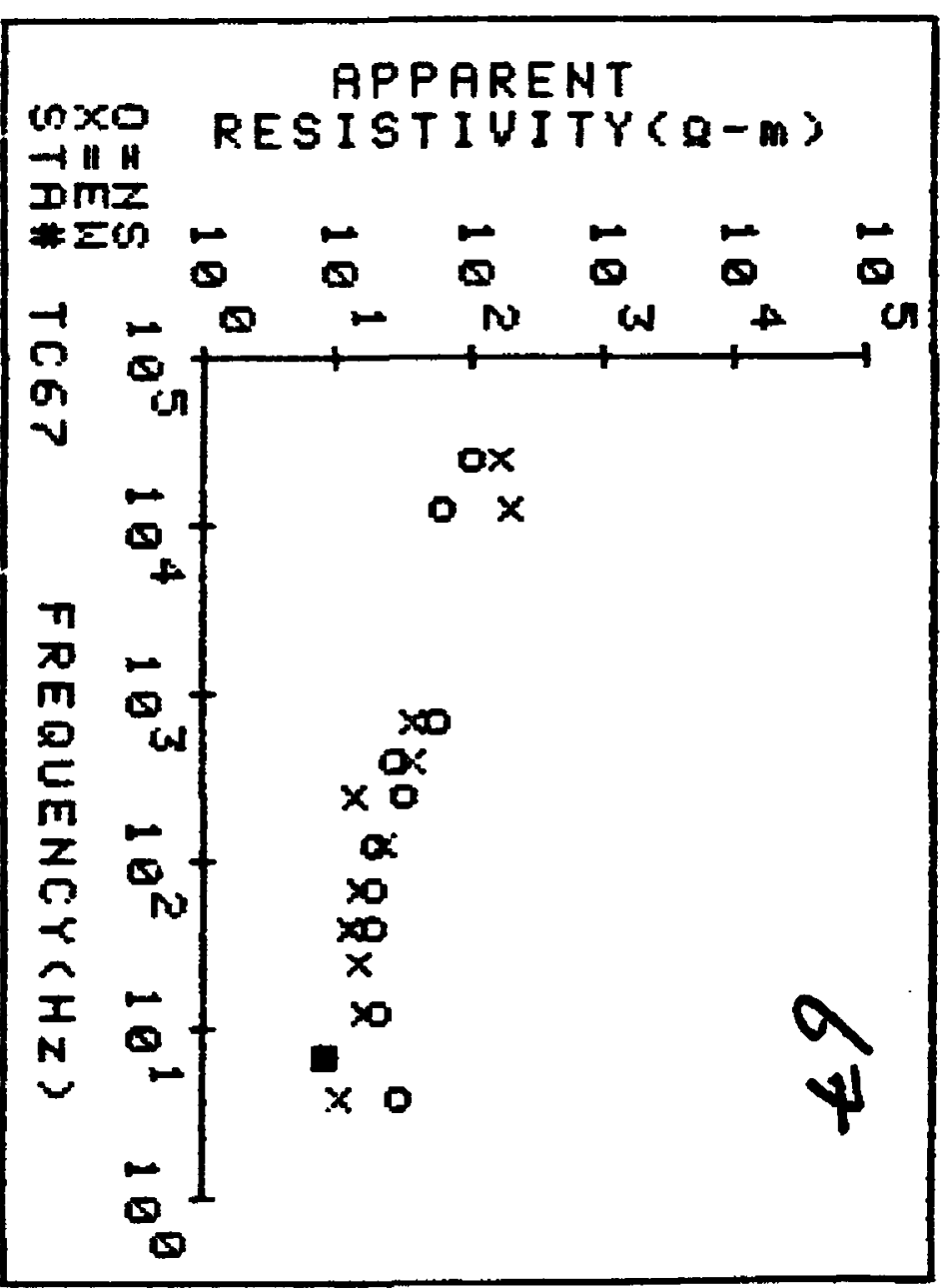

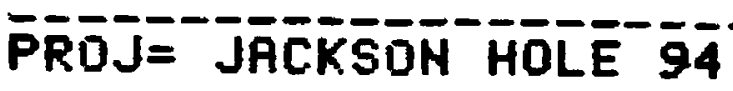

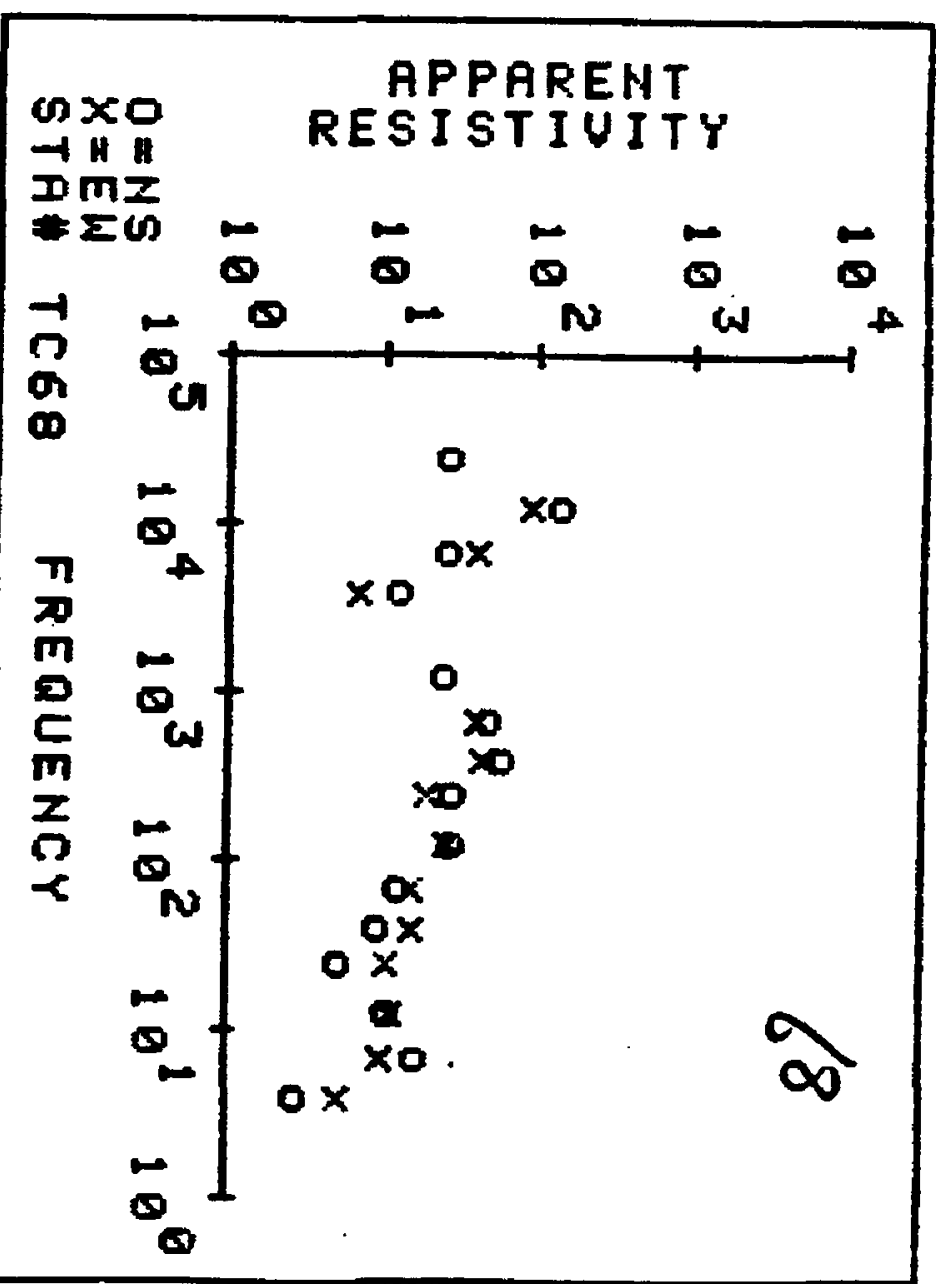




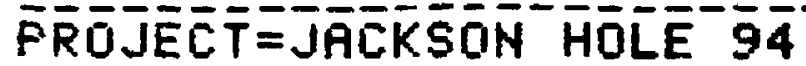

STA. IU_TCES NS ND FPEQ $=14$

\begin{tabular}{|c|c|c|c|}
\hline $\begin{array}{r}\text { FREQ } \\
4.5 \\
7.5 \\
14.0 \\
27.0 \\
45.0 \\
75.0 \\
140.0 \\
270.0 \\
450.0 \\
750.0 \\
590.0 \\
590.0 \\
699.0\end{array}$ & $\begin{array}{r}\text { AP-RES } \\
9.62 \\
29.94 \\
19.99 \\
54.23 \\
52.35 \\
39.90 \\
21.11 \\
25.31 \\
46.29 \\
29.19 \\
19.14 \\
19.65 \\
42.14 \\
57.99\end{array}$ & $\begin{array}{c}\text { N OBS } \\
3 \\
6 \\
7 \\
9 \\
10 \\
10 \\
10 \\
7 \\
19 \\
6 \\
10 \\
9 \\
5 \\
6\end{array}$ & $\begin{array}{r}\text { STD ERR } \\
.51 \\
9.77 \\
9.43 \\
5.94 \\
3.19 \\
3.92 \\
1.75 \\
2.89 \\
4.47 \\
.95 \\
2.57 \\
2.55 \\
5.99 \\
3.24\end{array}$ \\
\hline
\end{tabular}

STA. ID_TC69 EW NO FREQ $=16$

FREQ AP-RES $N$ OES

$\begin{array}{rrr}4.5 & 9.31 & 3 \\ 7.5 & 3.79 & 9 \\ 4.0 & 11.69 & 10 \\ 7.0 & 53.11 & 10 \\ 4.9 & 59.77 & 19 \\ 5.9 & 66.87 & 11 \\ 9.0 & 29.16 & 10 \\ 0.0 & 31.64 & 10 \\ 6.0 & 29.99 & 9 \\ 0 & 39.99 & 9 \\ 9.9 & 31.14 & 3 \\ 0 & 31.54 & 4 \\ 0 & 9.92 & 9 \\ 0 & 34.92 & 11 \\ 0.0 & 76.61 & 6 \\ 0 & 33.14 & 5\end{array}$

STD ERR

.19

1.19

10.59

5.69

3.25

2.94

2.27

5.22

2.35

2.59

3.25

1.51

259

1. 30

10.53

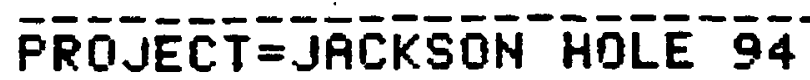

STA. ID_TC70 NS NO FREQ $=16$

$\begin{array}{rrrr}\text { FREQ } & \text { AP-RES } & \text { N OBS } & \text { STD ERR } \\ 4.5 & 5.91 & 3 & .29 \\ 7.5 & 7.98 & 5 & .41 \\ 14.0 & 27.88 & 5 & 5.12 \\ 27.8 & 29.79 & 6 & 5.26 \\ 45.0 & 33.29 & 10 & 1.95 \\ 75.9 & 17.51 & 19 & 2.33 \\ 149.0 & 13.17 & 11 & .85 \\ 270.0 & 6.42 & 9 & 3.48 \\ 459.9 & 18.64 & 8 & 7.89 \\ 750.0 & 53.82 & 19 & 7.64 \\ 1400.0 & 29.30 & 3 & 7.18 \\ 4509.0 & 47.69 & 3 & 1.29 \\ 7509.0 & 22.37 & 6 & 1.28 \\ 4090.0 & 75.88 & 3 & 7.63 \\ 7000.0 & 45.18 & 4 & 1.67 \\ 7000.0 & 56.92 & 3 & 1.39\end{array}$

STA. ID_TC7O EW NO FREQ $=13$

FREQ RP-RES N OBS STD ERR $4.5 \quad 69.68$

$7.5 \quad 38.43$

14.9

27.9

40.92

4.5 .8

48.45

36.90

75.0

37.84

18.20

140.0

450.0

750.0

9.63

37.23

7.82

4500.0

7500.0

14900.0

8. 98

4.19

41.53

OB5
3
3
7
9
9
7
10
9
4
5
3
4
3

22.39

2. 87

4.64

4.72

3.93

.98

3.52

1.49

.75

.93

.53

.11

.13

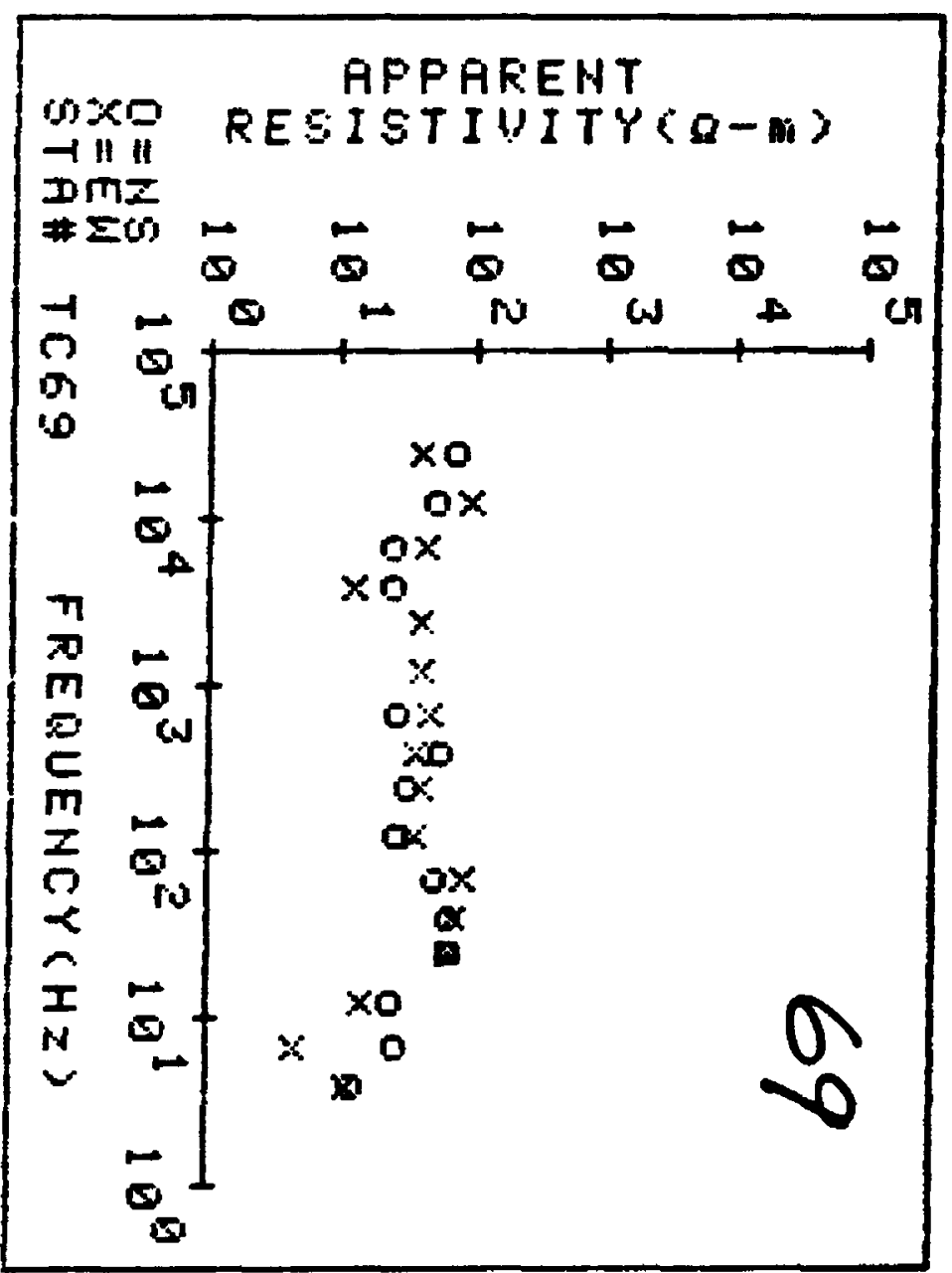

APPARENT

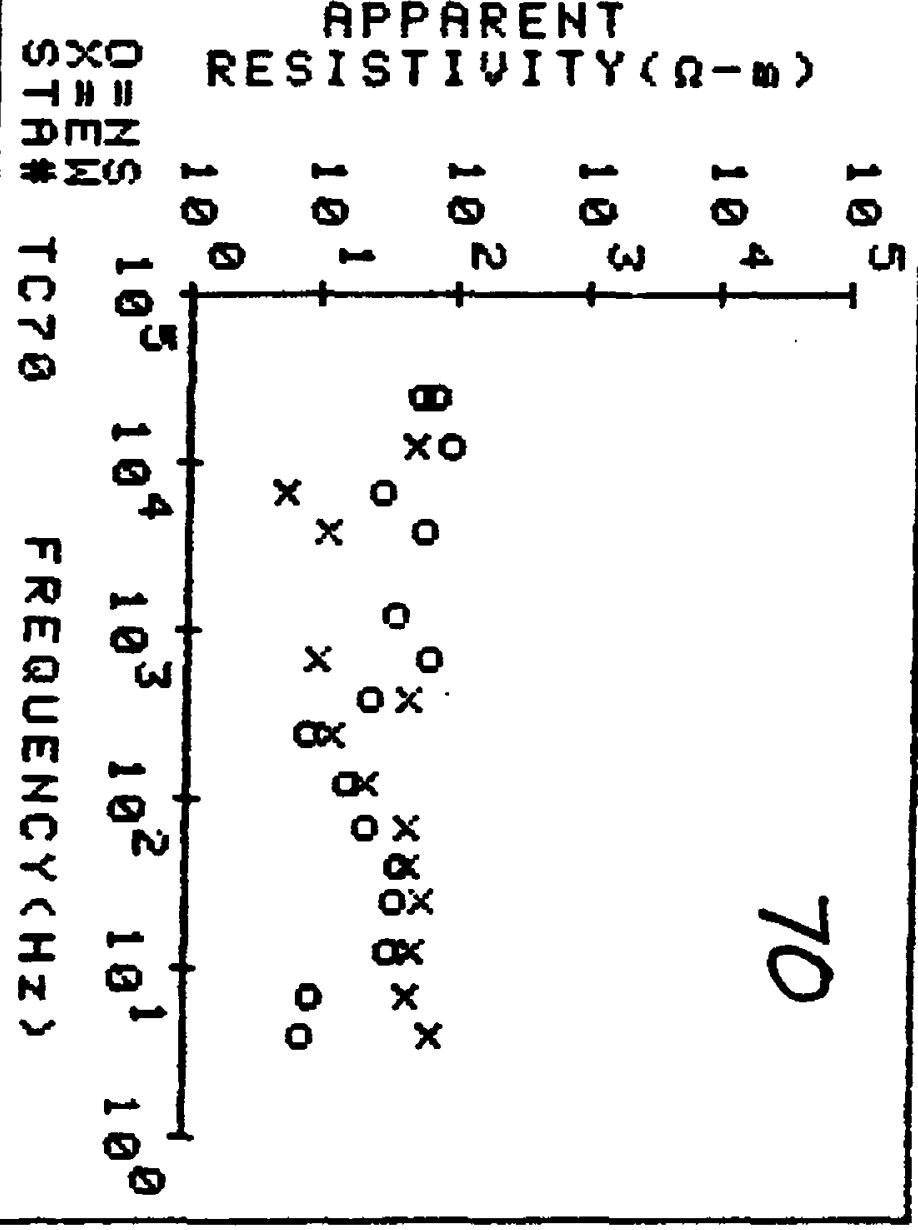


PROJJECT=JĀCKSON HOLE 94

STA. ID_TCPI NS NO FREQ $=13$

FREQ AF-RES H OBS STD ERR

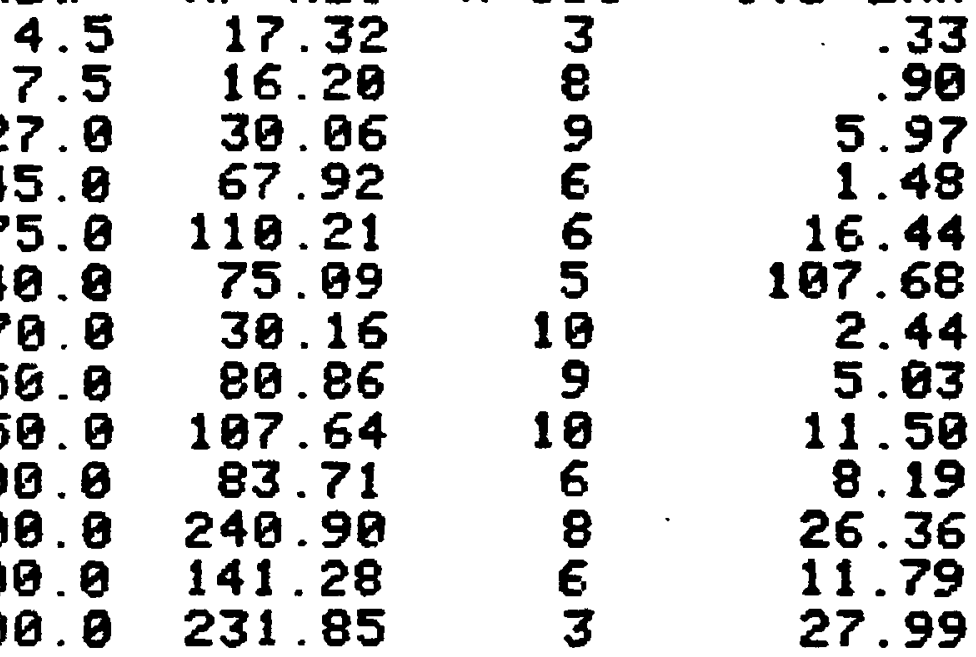

14080.0

STA. ID_TCPI EW NO FREQ $=15$

FREQ AP-RES N OBS STO ERR

4.584 .55

$7.5 \quad 188.82$

$14.0 \quad 88.91$

27.87 .17

$45.0 \quad 144.92$

75.0317 .98

140.0140 .45

270.0130 .10

450.0145 .37

$759.8 \quad 87.27$

$2700.0 \quad 306.95$

$4590.0 \quad 89.23$

$7506.0 \quad 253.22$

$14000.0 \quad 337.01$

$27000.0 \quad 30.75$

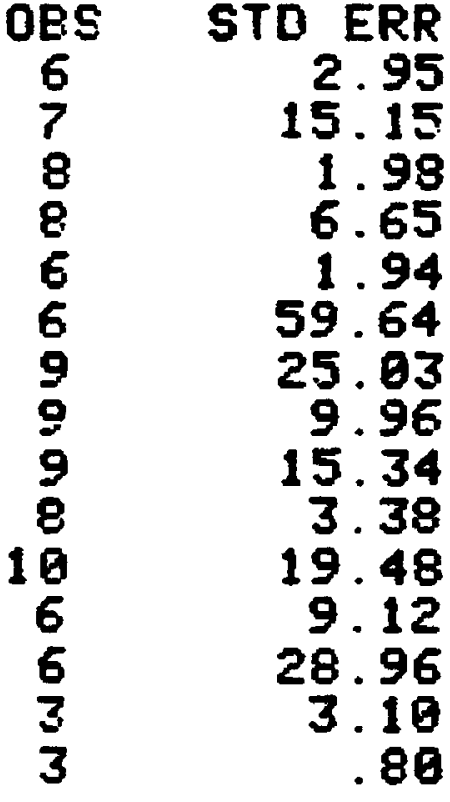

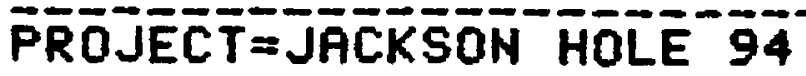

STA. ID_TCP2 NS NO FREQ $=12$

FREQ AP-RES N OBS STD ERP $4.5 \quad 185.42$

27.0189 .84

45.0134 .34

$75.0 \quad 255.58$

$140.0 \quad 174.99$

$278.0 \quad 286.34$

$450.0 \quad 459.66$

$750.0 \quad 517.50$

1400.0633 .47

$7500.0 \quad 469.23$

14000.0912 .07

27000.01855 .80

3
6
7
9
10
11
8
9
3
5
3
1

16.84

11.09

29.15

29.11

18.14

23.80

10.14

49.47

15.61

21.43

90.26

0.60

\section{STA. ID_TCP2 EN NO FREQ $=11$}

FREQ AP-RES $N$ OBS STD ERR

$\begin{array}{llll}4.5 & 134.14 & 3 & 15.44\end{array}$

$\begin{array}{llll}7.5 & 384.27 & 5 & 12.09\end{array}$

$\begin{array}{llll}14.0 & 114.62 & 6 & 4.27\end{array}$

$\begin{array}{llll}45.8 & 305.88 & 6 & 4.17\end{array}$

$\begin{array}{llll}75.0 & 73.58 & 8 & 13.11\end{array}$

$\begin{array}{llll}140.6 & 121.95 & 11 & 24.29\end{array}$

$\begin{array}{llll}270.0 & 99.67 & 8 & 26.32\end{array}$

$\begin{array}{llll}750.0 & 218.90 & 5 & 3.31\end{array}$

$\begin{array}{llll}7509.0 & 96.27 & 8 & 18.82\end{array}$

$7500.0 \quad 94.29 \quad 5 \quad 5.82$

$\begin{array}{llll}27000.0 & 13.73 & 1 & 0.00\end{array}$
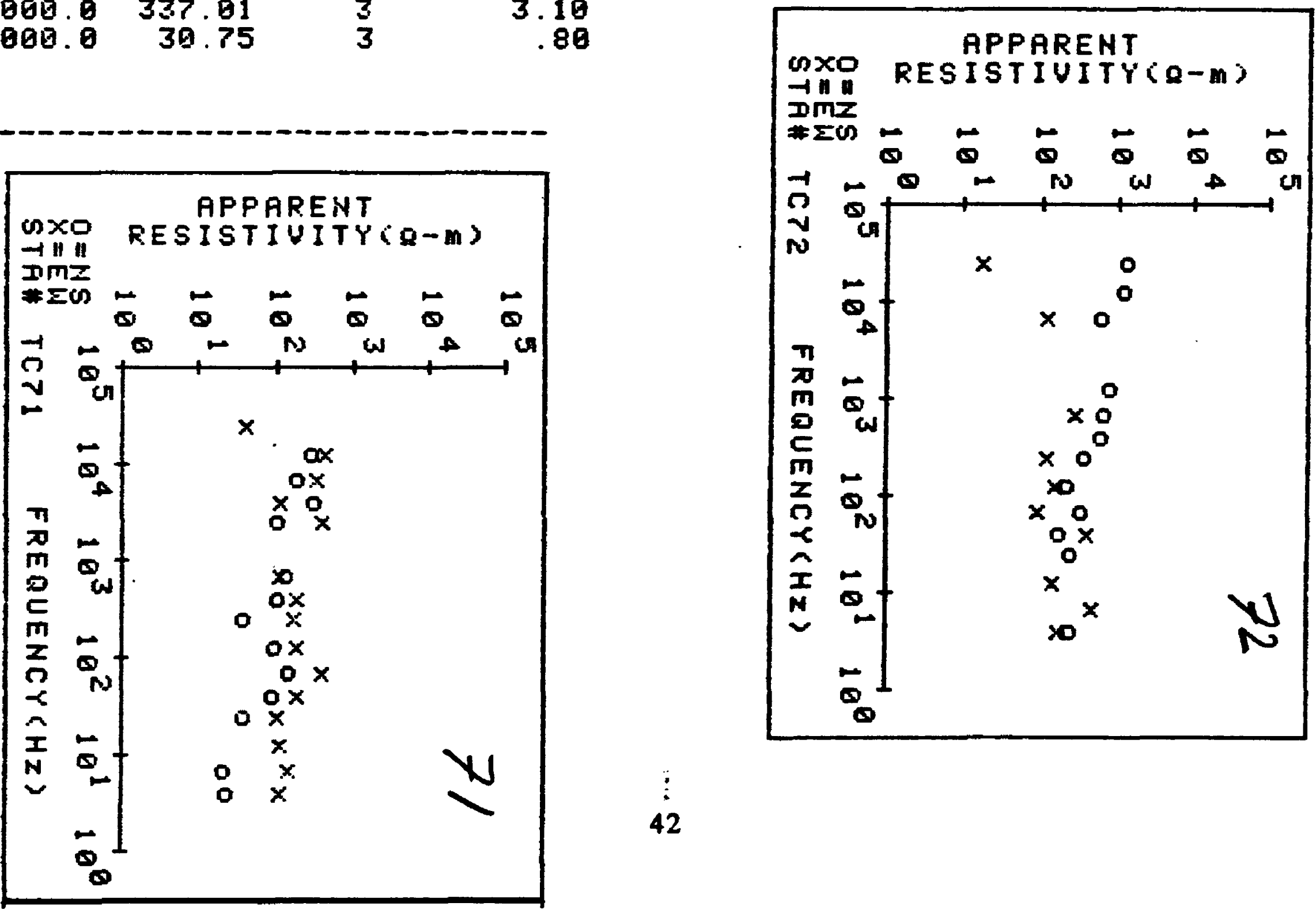
PROJECT =JATKSON HOLE 94

STF. ID_TCP3 NS NO FPEQ $=12$

\begin{tabular}{|c|c|c|c|}
\hline $\begin{array}{r}\text { FREQ } \\
7.5 \\
14.9 \\
27.9 \\
45.9 \\
75.9 \\
149.9 \\
279.9 \\
459.9 \\
499.9 \\
596.9 \\
999.9\end{array}$ & $\begin{array}{l}\text { AP-RES } \\
109.13 \\
132.53 \\
111.46 \\
557.99 \\
473.94 \\
612.95 \\
336.24 \\
193.01 \\
679.43 \\
149.20 \\
177.45 \\
39.58\end{array}$ & $\begin{array}{c}\text { N OES } \\
3 \\
5 \\
8 \\
7 \\
7 \\
7 \\
8 \\
8 \\
7 \\
4 \\
6 \\
6 \\
3 \\
1\end{array}$ & $\begin{array}{r}\text { STD EFR } \\
.80 \\
2.44 \\
25.12 \\
99.99 \\
26.11 \\
95.75 \\
43.29 \\
42.55 \\
22.65 \\
35.28 \\
11.49\end{array}$ \\
\hline
\end{tabular}

STA. ID_TC.T3 EH NO FPEQ $=11$

FREQ AF-RES N OBS STD ERR

$\begin{array}{rrrr}7.5 & 85.97 & 3 & 1.76 \\ 14.9 & 390.97 & 4 & 15.74 \\ 27.9 & 395.25 & 3 & 16.99 \\ 75.9 & 333.52 & 8 & 87.93 \\ 49.9 & 495.93 & 8 & 22.34 \\ 70.9 & 275.56 & 8 & 40.98 \\ 50.9 & 485.48 & 7 & 39.96 \\ 59.9 & 181.35 & 5 & 29.93 \\ 99.0 & 465.13 & 4 & 13.51 \\ 99.9 & 139.42 & 3 & 17.77 \\ 90.9 & 78.74 & 1 & 9.90\end{array}$

14969.9
27969.9
PROJECTT=JACKSON HOLE $9 \overline{4}$

STA. ID_TC74 NS NO FREQ $=11$

FREQ AP-RES N OES STD ERR $\begin{array}{lllll}4.5 & 332.96 & 4 & 22.52\end{array}$

$\begin{array}{llll}7.5 & 341.97 & 3 & 32.59\end{array}$

$14.0 \quad 678.03 \quad 4 \quad 47.81$

$27.0 \quad 949.76 \quad 4 \quad 165.94$

75.0693 .52

14901935.29

759.01684 .60

$4509.6 \quad 422.49$

$7599.9 \quad 66.85$

14990.0296 .26

$27000.9 \quad 52.47$

$6 \quad 191.81$

$6 \quad 138.26$

$4 \quad 59.81$

$6 \quad 36.18$

42.24

$3 \quad 17 \cdot 20$

3

1.15

STA. ID_TC74 EN NO FREQ $=12$

FREQ AF-RES N OES STD EFP $7.5 \quad 169.83$

27.91945 .19

45.9399 .73

75.0741 .89

140.890 .90

279.0283 .69

450.0232 .93

$7590 \quad 222.51$

$2700.0 \quad 149.29$

$4509.0 \quad 79.44$

$7500.0 \quad 21.42$

$14999.0 \quad 26.63$
2.00 320.71

42.23

95.47

54.61

14.11

34.10

7.67

16.67

10. 87

1.93
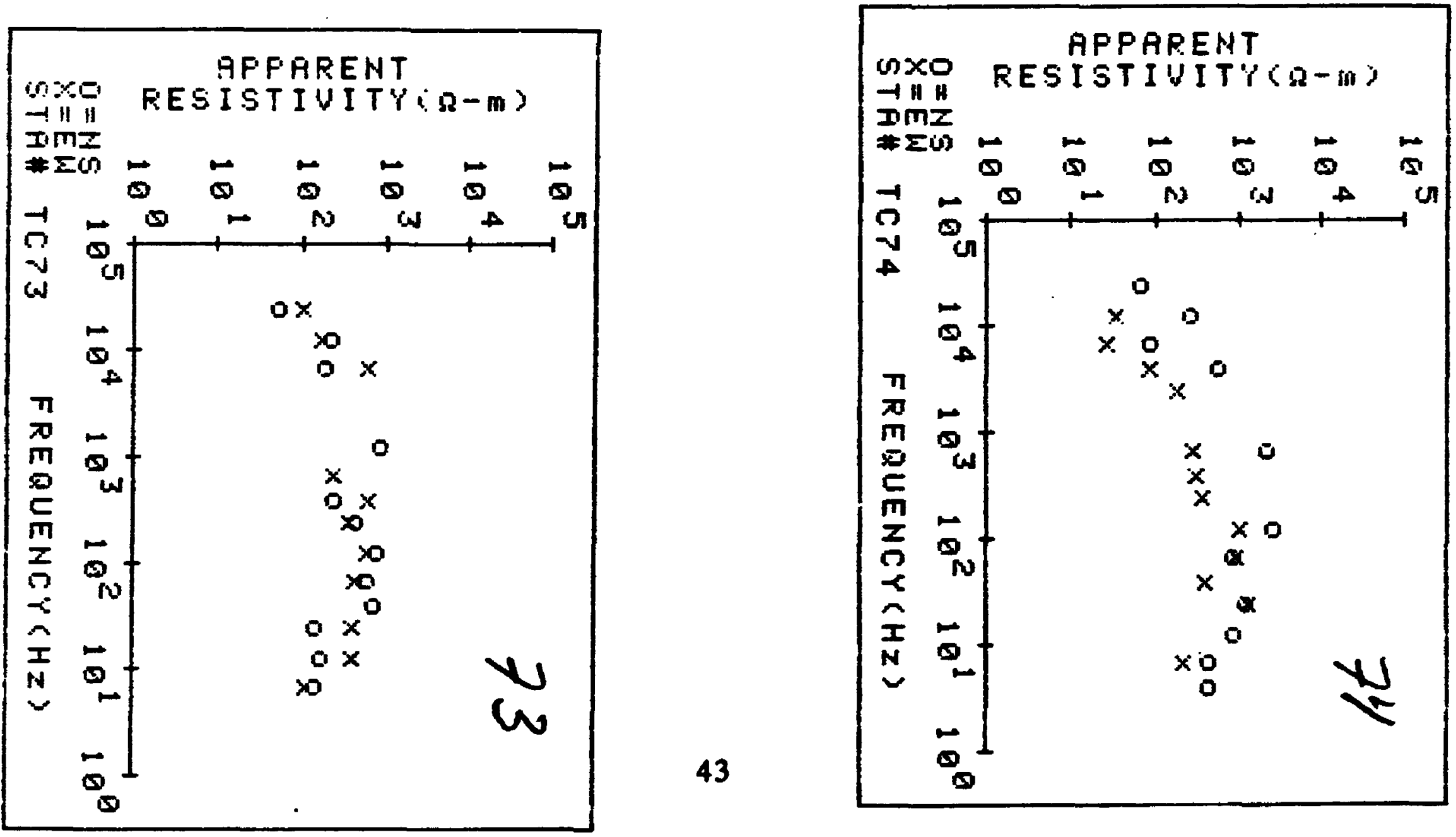
PROJ= JFICKSON HOLE 94

5TA-IOTC75FILE NAME $=$ TC75

F̄ROJEC̄T=JĀCKSON HOLE

STA. IO_TC75 NS NO FREQ $=12$

\begin{tabular}{|c|c|c|c|c|}
\hline $\begin{array}{r}\text { FREQ } \\
7.5 \\
14.0 \\
27.0 \\
45.9 \\
75.9 \\
149.9 \\
270.0 \\
450.0 \\
509.9 \\
500.0 \\
909\end{array}$ & $\begin{array}{r}\text { AF-RES } \\
84.94 \\
189.79 \\
286.89 \\
76.19 \\
319.94 \\
383.49 \\
24.33 \\
42.77 \\
31.72 \\
26.20 \\
51.11 \\
28.57\end{array}$ & N C & $\begin{array}{c}08 S \\
5 \\
5 \\
8 \\
7 \\
7 \\
8 \\
6 \\
8 \\
10 \\
9 \\
3 \\
1\end{array}$ & $\begin{array}{r}\text { STD ERR } \\
8.37 \\
16.50 \\
50.20 \\
1.72 \\
14.29 \\
17.21 \\
1.28 \\
8.20 \\
2.65 \\
1.94 \\
1.26\end{array}$ \\
\hline
\end{tabular}

STA: IO_TC75 EW NO FREQ $=9$

$\begin{array}{rrrr}\text { FREQ } & \text { AP-RES } & \text { N OBS } & \text { STD ERR } \\ 7.5 & 45.54 & 6 & 3.00 \\ 14.0 & 49.19 & 4 & 1.07 \\ 27.0 & 171.56 & 6 & 16.24 \\ 45.0 & 42.41 & 7 & 8.65 \\ 149.0 & 300.56 & 9 & 13.78 \\ 279.9 & 199.36 & 11 & 13.24 \\ 450.0 & 67.33 & 8 & 12.93 \\ 7590.0 & 14.77 & 7 & .37 \\ 4090.0 & 19.89 & 3 & .45\end{array}$

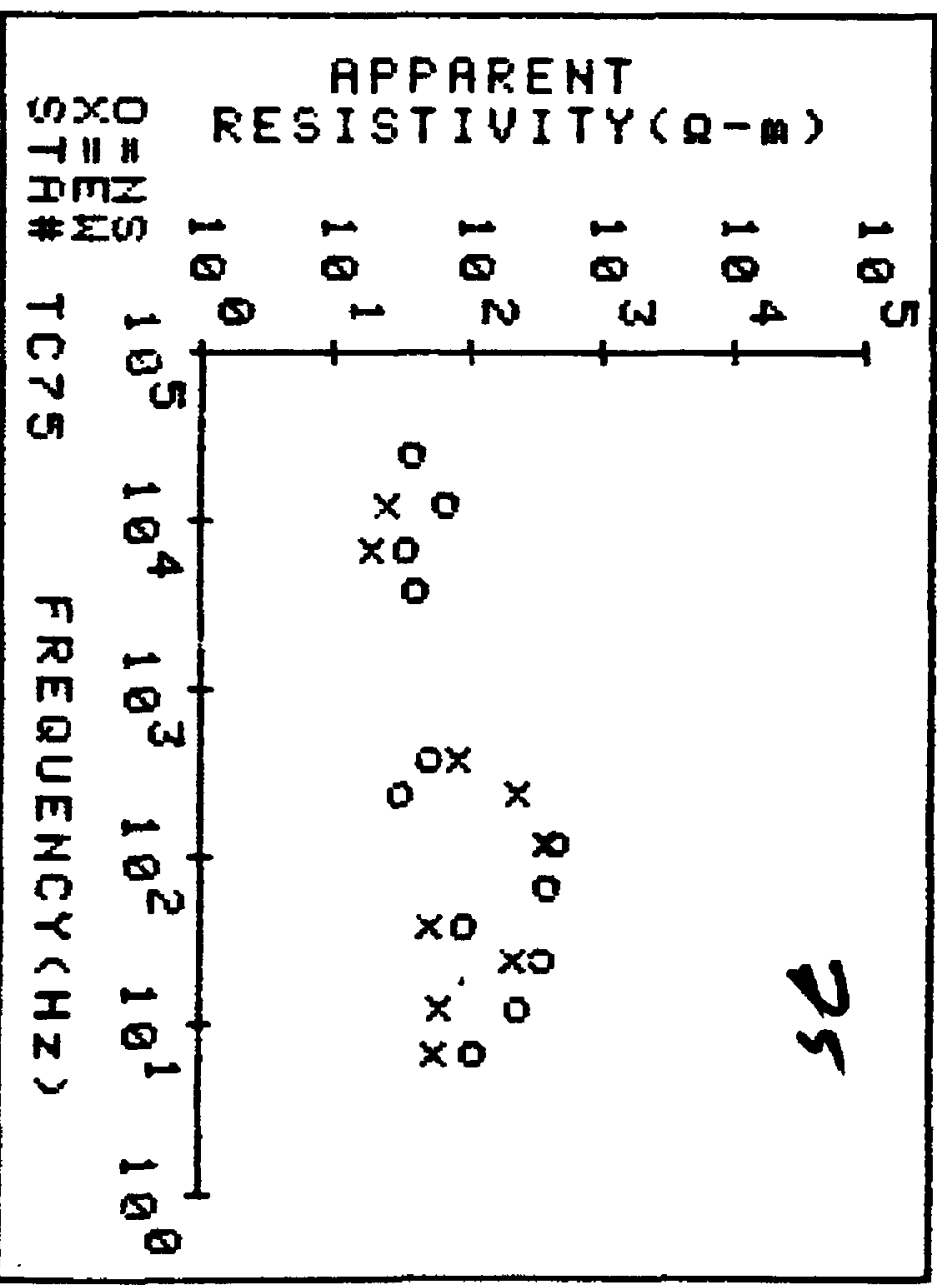

\section{PROOJECT =JACKSON HOLE 94}

STA. ID_TC76 NS NO FREQ $=9$

\begin{tabular}{rrcr}
\multicolumn{1}{r}{ FREQ } & AP-RES & $N$ OBS & STD ERR \\
4.5 & 2088.09 & 4 & 84.53 \\
7.5 & 675.70 & 3 & 60.52 \\
14.0 & 295.99 & 3 & 24.55 \\
27.0 & 353.97 & 3 & 29.38 \\
45.0 & 1173.19 & 4 & 18.21 \\
2790.0 & 709.94 & 6 & 152.06 \\
7500.0 & 68.66 & 3 & 3.52 \\
4900.0 & 53.50 & 3 & 2.56 \\
7000.0 & 26.54 & 1 & 0.00
\end{tabular}

STA. ID_TC76 EH NO FREQ $=8$

FREQ AF-RES $N$ OBS STL ERR $27.0260 .27 \quad 5 \quad 5.18$ $75.0 \quad 181.46 \quad 8 \quad 22.07$

$\begin{array}{llll}140.9 & 103.17 & 9 & 14.50\end{array}$

$270.0160 .32 \quad 4 \quad .80$

$\begin{array}{llll}450.0 & 60.22 & 7 & 1.83\end{array}$

$\begin{array}{llll}750.0 & 60.17 & 7 & 2.23\end{array}$

$\begin{array}{rrrr}14000.9 & 130.68 & 3 & 1.84 \\ 27000.0 & 22.64 & 3 & 1.18\end{array}$

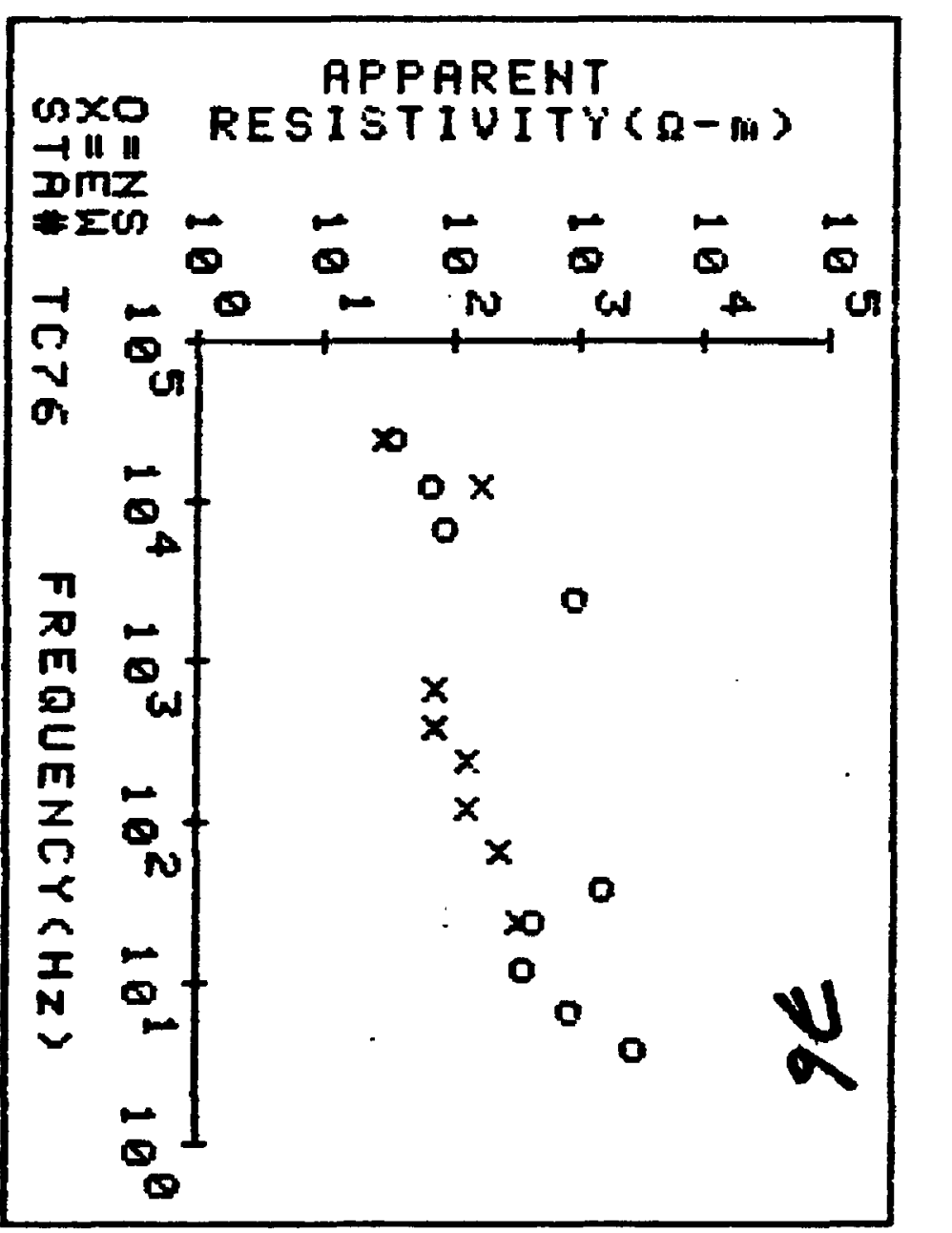


STA. ID_TC77 NS NO FREQ $=14$

$\begin{array}{rrrr}\text { FREQ } & \text { RP-RES } & \text { N OBS } & \text { STO ERR } \\ 4.5 & 38.77 & 3 & 2.04 \\ 7.5 & 31.79 & 8 & 1.05 \\ 14.0 & 50.09 & 5 & 15.48 \\ 27.0 & 43.20 & 8 & 16.30 \\ 45.0 & 35.81 & 8 & 5.20 \\ 75.0 & 38.42 & 9 & 4.90 \\ 140.0 & 31.05 & 9 & 2.99 \\ 270.0 & 27.91 & 9 & 3.83 \\ 750.0 & 57.35 & 8 & 5.28 \\ 2709.0 & 51.27 & 5 & 1.43 \\ 4500.0 & 23.84 & 10 & 3.82 \\ 500.0 & 24.64 & 6 & 1.59 \\ 4600.0 & 97.35 & 3 & 9.30 \\ 000.0 & 33.92 & 1 & 0.00\end{array}$

STR. ID_TCT7 EH NO FREQ $=15$

FREQ AP-RES N OBS STD ERR

$4.5 \quad 8.5 .50$

$7.5 \quad 52.04$

14.060 .61

27.090 .62

45.0

75.9

140.0

270.0

450.0

750.0

2700.0

4506.0

7500.0

14000.0

27060.0
44.93

57.68

64.74

56.94

89.25

74.07

144.26

69.75

60.71

182.64

129.69

$\begin{array}{rr}5 & 9 \cdot 24 \\ 9 & 3 \cdot 11 \\ 4 & 18.59 \\ 5 & 21.75 \\ 3 & 15.47 \\ 7 & 5 \cdot 28 \\ 10 & 14.76 \\ 8 & 3.59 \\ 8 & 13 \cdot 38 \\ 10 & 3.93 \\ 8 & 12.77 \\ 7 & 5 \cdot 37 \\ 5 & 7.71 \\ 3 & 2.55 \\ 1 & 0.90\end{array}$

APPARENT

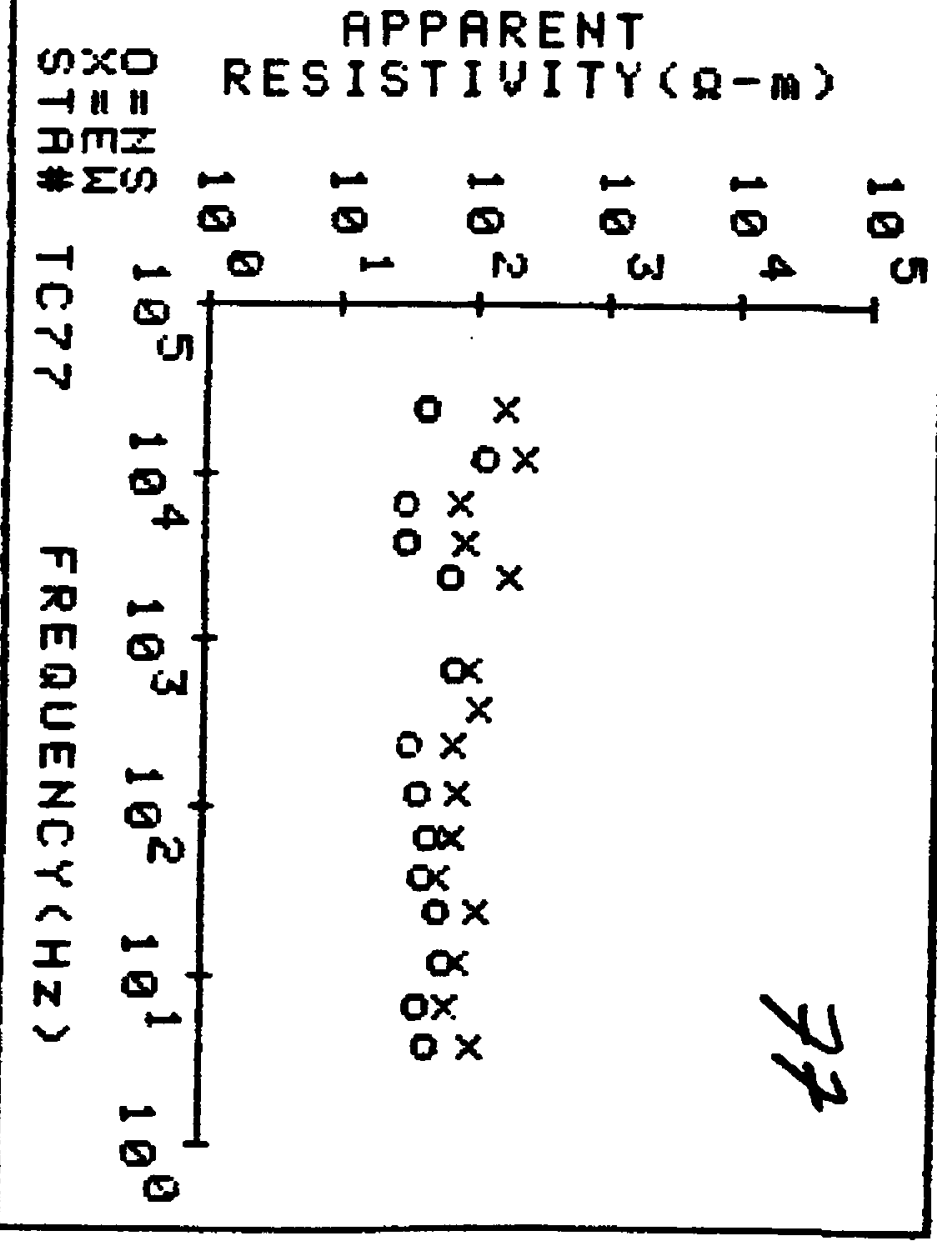

PROJ= JACKSON HOLE 94

STA-IDTCP8FILE NAME $=$ TC78

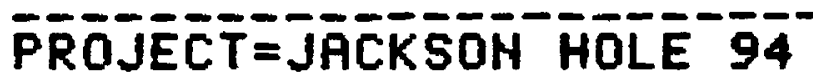

STA. ID_TC78 NS NO FREQ $=14$

$\begin{array}{rrrr}\text { FREQ } & \text { AP-RES } & \text { N OBS } & \text { STD ERR } \\ 4.5 & 128.26 & 7 & 4.75 \\ 7.5 & 60.51 & 3 & 3.38 \\ 27.0 & 47.08 & 6 & 2.00 \\ 45.0 & 33.19 & 8 & 3.83 \\ 75.0 & 42.45 & 9 & 4.16 \\ 140.6 & 54.04 & 7 & 2.09 \\ 270.0 & 37.93 & 8 & 3.72 \\ 450.0 & 45.85 & 8 & 3.88 \\ 750.0 & 95.99 & 8 & 9.28 \\ 1409.0 & 30.98 & 8 & 1.88 \\ 2700.0 & 49.64 & 10 & 3.10 \\ 4509.0 & 94.59 & 7 & 5.33 \\ 7580.0 & 59.32 & 8 & 3.82 \\ 7000.0 & 34.43 & 4 & 1.17\end{array}$

5TA. ID_TC78 EH HO FREQ $=12$

FREQ AP-RES N OES STD ERR

$14.0 \quad 43.11 \quad 1 \quad 0.00$

$\begin{array}{llll}27.0 & 48.27 & 6 & 4.55\end{array}$

$45.0 \quad 34.59 \quad 9 \quad 11.99$

$\begin{array}{llll}75.0 & 24.74 & 8 & 1.94\end{array}$

$140.0 \quad 15.67 \quad 8 \quad 1.57$

$\begin{array}{llll}270.0 & 17.72 & 8 & 1.78\end{array}$

$\begin{array}{llll}450.0 & 22.37 & 8 & 2.47\end{array}$

$\begin{array}{llll}750.0 & 17.12 & 8 & 2.16\end{array}$

$\begin{array}{llll}2700.0 & 26.10 & 3 & 1.06\end{array}$

$\begin{array}{llll}4500.0 & 35.10 & 11 & 4.54\end{array}$

$\begin{array}{llll}7500.0 & 43.25 & 9 & 1.48\end{array}$

$14800.0 .45 .30 \quad 3 \quad 4.87$

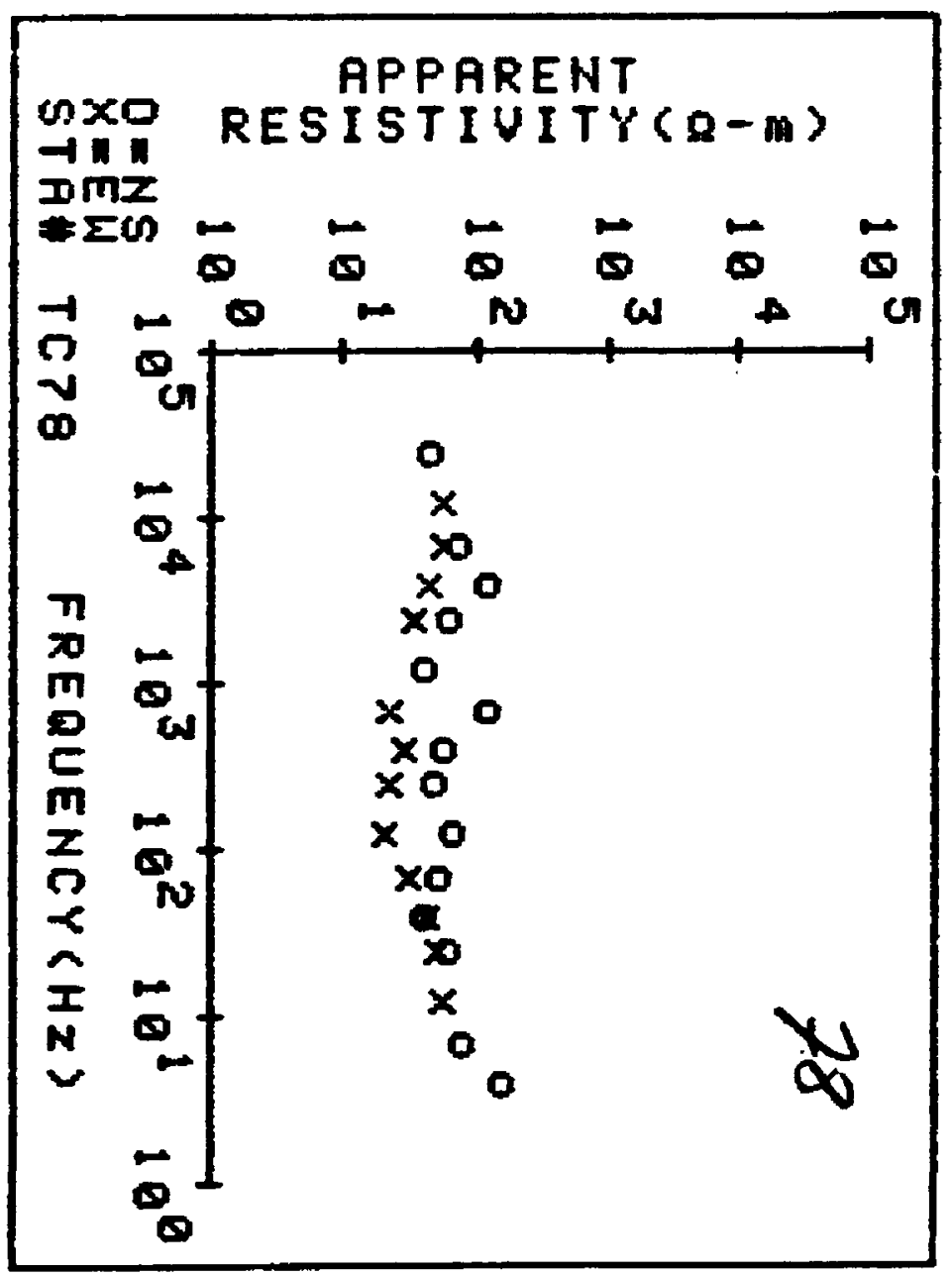


PROJECT=JACKSON HOLE 94

PROJJECTEJĀCKSONN HOLE

STA. ID_TCP9 NS NO FREQ=?

$\begin{array}{rrcr}\text { FREQ } & \text { AF-RES } & \text { N OES } & \text { STO ERR } \\ 4.5 & 113.40 & 1 & 9.90 \\ 7.5 & 97.55 & 4 & 6.20 \\ 140.6 & 71.65 & 6 & 8.71 \\ 450.0 & 96.79 & 6 & 3.49 \\ 750.0 & 84.13 & 7 & 1.15 \\ 7590.0 & 189.53 & 7 & 9.40 \\ 7990.0 & 148.82 & 3 & 3.91\end{array}$

STA. ID_TC79 EH NO FREQ $=12$

FREQ AP-RES N OBS STD ERR $4.5 \quad 285.68$

7.5206 .41

$27.0 \quad 265.76$

75.194 .49

$140.0 \quad 148.31$

450.0295 .62

750.0167 .18

2790.154 .81

4509.193 .11

$7500.0 \quad 89.82$

14990.0

27000.

176.80

47.25

3
3
6
8
6
7
6
5
4
7
3
4

16.67

38.35

2.72

18.25

42.75

13.73

60.94

3.20

15.24

5.91

5.95

2.87

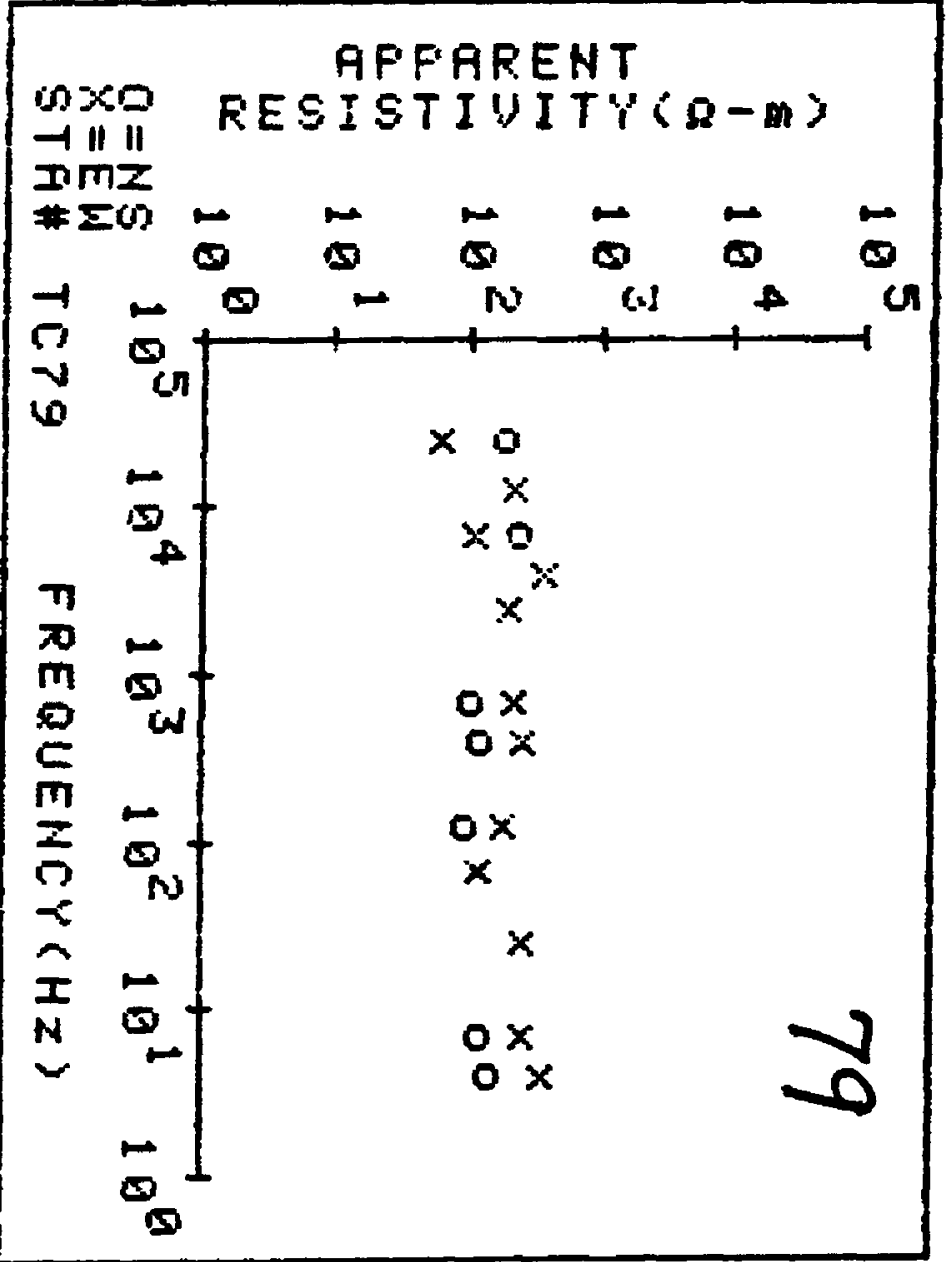

STA. ID_TCSO NS NO FREQ $=16$

\begin{tabular}{|c|c|c|c|}
\hline $\begin{array}{r}\text { FREQ } \\
4.5 \\
7.5 \\
14.0 \\
27.0 \\
45.0 \\
75.0 \\
140.0 \\
270.0 \\
450.0 \\
750.0 \\
460.0\end{array}$ & $\begin{array}{r}\text { AP-RES } \\
56.23 \\
46.74 \\
76.29 \\
178.59 \\
244.81 \\
321.16 \\
188.57 \\
162.68 \\
490.41 \\
351.46 \\
330.25 \\
329.41 \\
102.85 \\
185.03 \\
181.57 \\
28.89\end{array}$ & $\begin{array}{c}\text { N OBS } \\
6 \\
5 \\
8 \\
11 \\
6 \\
19 \\
19 \\
19 \\
11 \\
8 \\
4 \\
5 \\
8 \\
19 \\
-4 \\
4\end{array}$ & $\begin{array}{r}\text { STDEF } \\
5.4 \\
1.6 \\
6.4 \\
13.5 \\
7.3 \\
96.2 \\
24.3 \\
12.6 \\
46.5 \\
38.2 \\
35.2 \\
27.6 \\
31.4 \\
21.9\end{array}$ \\
\hline
\end{tabular}

STA. ID_TC8O EW NO FREQ $=16$

\begin{tabular}{|c|c|c|c|c|}
\hline $\begin{array}{r}\text { FREQ } \\
4.5 \\
7.5 \\
14.0 \\
27.0 \\
45.0 \\
75.0 \\
140.0 \\
270.0 \\
450.0 \\
750.0 \\
400.0 \\
790.0 \\
500.0 \\
500.0 \\
000.0\end{array}$ & $\begin{array}{r}\text { AP-RES } \\
33.45 \\
20.15 \\
33.19 \\
28.07 \\
59.26 \\
79.23 \\
193.58 \\
139.08 \\
358.47 \\
129.93 \\
157.47 \\
137.44 \\
219.06 \\
84.77 \\
155.33 \\
81.09\end{array}$ & N & $\begin{array}{r}0 B 5 \\
6 \\
10 \\
10 \\
9 \\
7 \\
10 \\
10 \\
10 \\
8 \\
7 \\
7 \\
8 \\
11 \\
4 \\
4 \\
9\end{array}$ & $\begin{array}{r}\text { STD ER } \\
3.5 \\
1.1 \\
2.0 \\
1.9 \\
3.0 \\
2.29 \\
6.6 \\
46.3 \\
11.31 \\
10.39 \\
3.18 \\
16.13 \\
3.26 \\
2.92 \\
10.73\end{array}$ \\
\hline
\end{tabular}

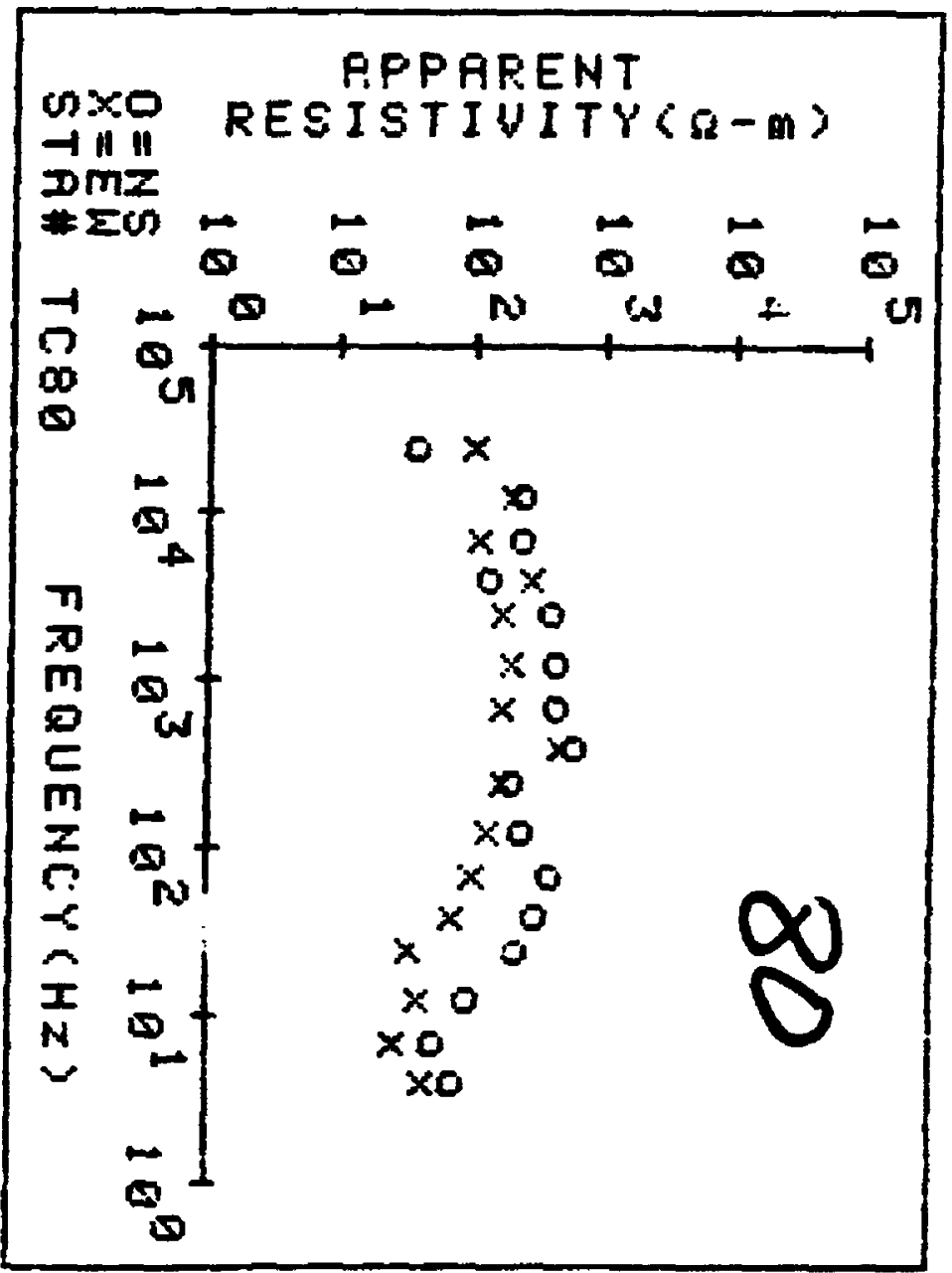




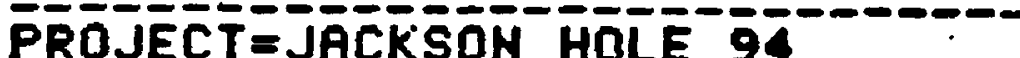

STA. ID_TCEI NS NO FREQ $=8$

$\begin{array}{rrrr}\text { FRER } & \text { AP-RES } & \text { N OES } & \text { STD ERR } \\ 14.8 & 23.19 & 4 & 7.60 \\ 27.8 & 28.72 & 2 & 1.22 \\ 270.8 & 58.55 & 4 & 28.87 \\ 758.8 & 27.58 & 4 & 4.22 \\ 2780.8 & 23.57 & 7 & 6.52 \\ 4580.8 & 35.74 & 2 & .15 \\ 7580.8 & 49.75 & 5 & 12.69 \\ 4080.8 & 76.63 & 1 & 8.68\end{array}$

STA. ID_TC81 EH HO FREQ $=8$

FREQ AP-RES N OBS STD ERR

$\begin{array}{rrrr}7.5 & 8.21 & 4 & 6.93 \\ 14.8 & 46.84 & 1 & 6.06 \\ 45.8 & 54.75 & 2 & 2.27 \\ 75.8 & 35.69 & 4 & 7.12 \\ 46.8 & 28.77 & 3 & .74 \\ 76.0 & 34.35 & 3 & 3.26 \\ 58.0 & 23.67 & 3 & .23 \\ 90.0 & 42.73 & 3 & .72\end{array}$

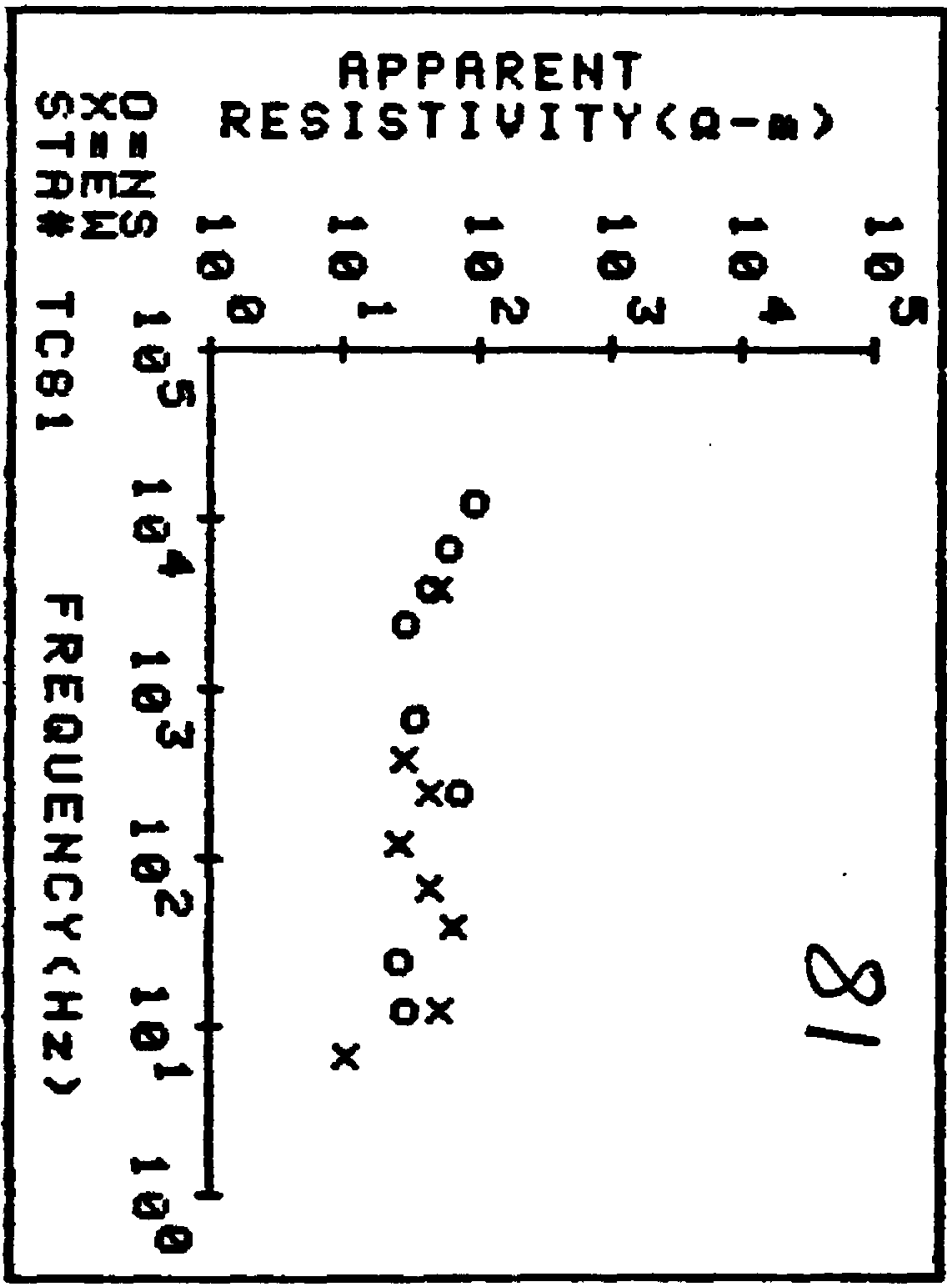

STA. ID_TC82 NS NO FREQ $=16$

\begin{tabular}{|c|c|c|}
\hline & $\begin{array}{r}\text { AP-RES } \\
169.13 \\
93.26 \\
362.44 \\
433.93 \\
438.15 \\
766.54 \\
369.62 \\
385.74 \\
306.53 \\
216.24 \\
229.48 \\
439.85 \\
280.73 \\
244.72 \\
157.89 \\
24.58\end{array}$ & $\begin{array}{c}\text { N O8S } \\
7 \\
7 \\
10 \\
19 \\
18 \\
22 \\
24 \\
4 \\
5 \\
6 \\
6 \\
6 \\
6 \\
18 \\
8 \\
17\end{array}$ \\
\hline
\end{tabular}

STA. ID_TC82 EH NO FREQ $=16$

\begin{tabular}{|c|c|c|c|}
\hline $\begin{array}{r}\text { FRER } \\
4.5 \\
7.5 \\
14.8 \\
27.8 \\
45.8 \\
75.8 \\
148.8 \\
278.8 \\
458.8 \\
750.0 \\
1408.8 \\
2700.0\end{array}$ & $\begin{array}{r}\text { AP-RES } \\
50.82 \\
63.47 \\
87.98 \\
205.98 \\
219.59 \\
326.24 \\
392.23 \\
358.97 \\
491.29 \\
512.68 \\
631.53 \\
379.13 \\
587.91 \\
772.38 \\
272.09\end{array}$ & $\begin{array}{c}\text { N OBS } \\
2 \\
7 \\
12 \\
12 \\
11 \\
11 \\
15 \\
12 \\
7 \\
7 \\
6 \\
9 \\
9 \\
6 \\
11 \\
18\end{array}$ & $\begin{array}{r}\text { STD ERR } \\
6.68 \\
15.86 \\
9.99 \\
46.26 \\
35.97 \\
34.91 \\
68.48 \\
17.98 \\
25.41 \\
78.78 \\
394.61 \\
58.08\end{array}$ \\
\hline
\end{tabular}

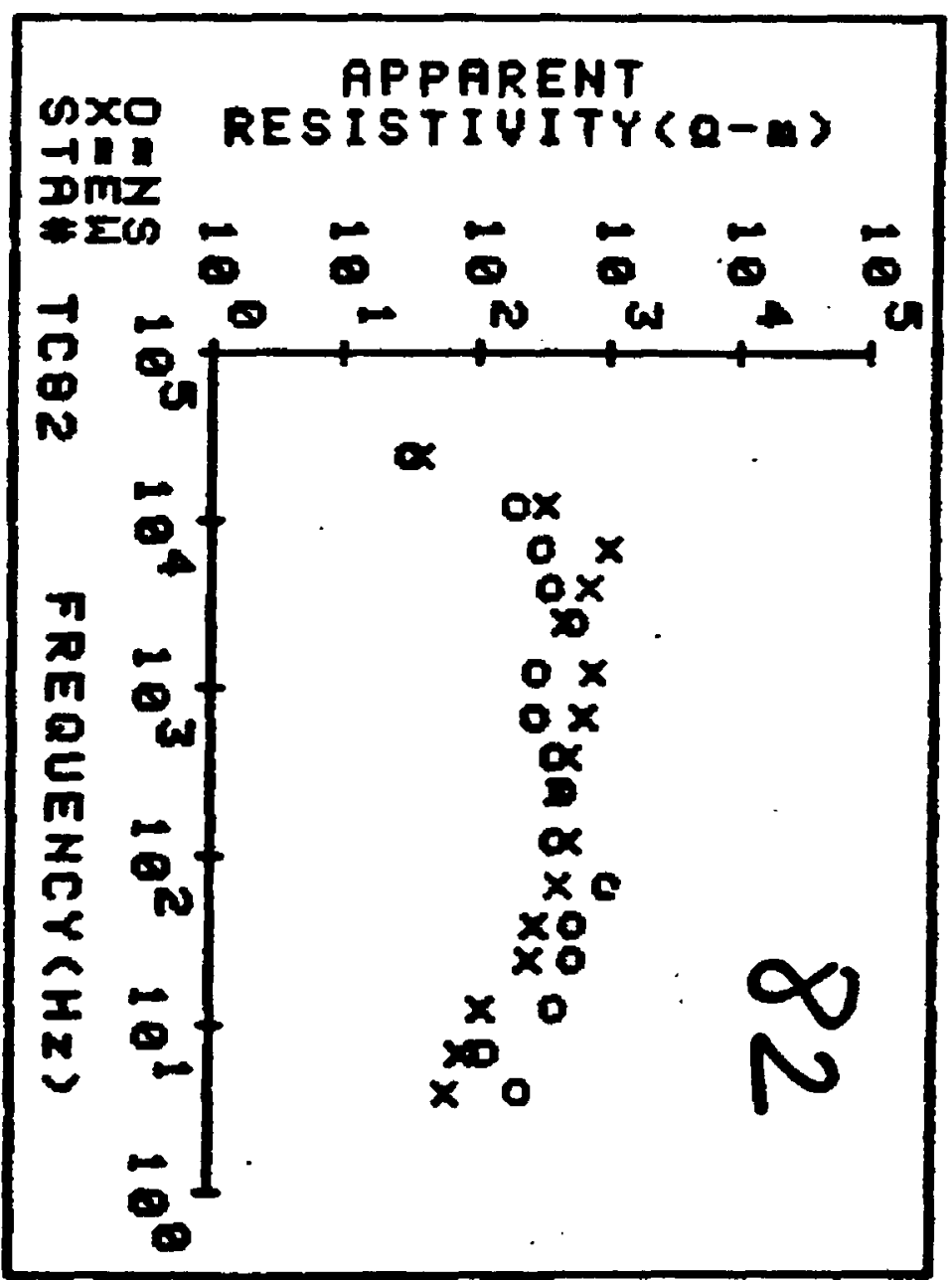


Appendix B

Bostick (1977) inversion interpretations of possible geoelectrical layers under AMT sounding sites in Jackson Hole, WY. For each sounding the $E-W$ and $N-S$ data from Appendix $A$ have been averaged and then smoothed and resampled at the original frequencies. The interpretation method that was used here calculates and plots one geoelectric layer for each pair of resampled data points. Not all such calculated layers are meaningful, but we expect the general trends shown on each plot to be valid. The first and last calculated points have been extended to the edge of the plot only to guide the eye; it should not be inferred that the plotted resistivity holds over that extended depth range.

The typed labels on each page are transcriptions of brief notes made in the field or during processing. They give written station locations specifying Quadrangle, Township, Range, and quarter-Section of quarter-Section (example: NESW S12 means the northeast quarter of the southwest quarter of Section 12), additional brief geographic descriptions, notes about field conditions that may have influenced data quality, and remarks about particular features, if any, that we noted about the data for the station. The data quality rating (Excellent, Good, Fair, Poor, Very Poor) is subjective, based on the opinion of the operator. A substantial separation between apparent resistivity curves drawn separately for NS and EW orientations can indicate strong lateral changes (a fault, for example) near the sounding station. The remarks accordingly attempt to indicate such separations. A remark of the type "NS below (above) EW" means that the apparent resistivity calculated from data using the NS electrode orientation is less than (more than) the apparent resistivity calculated from data using the EW electrode orientation. 


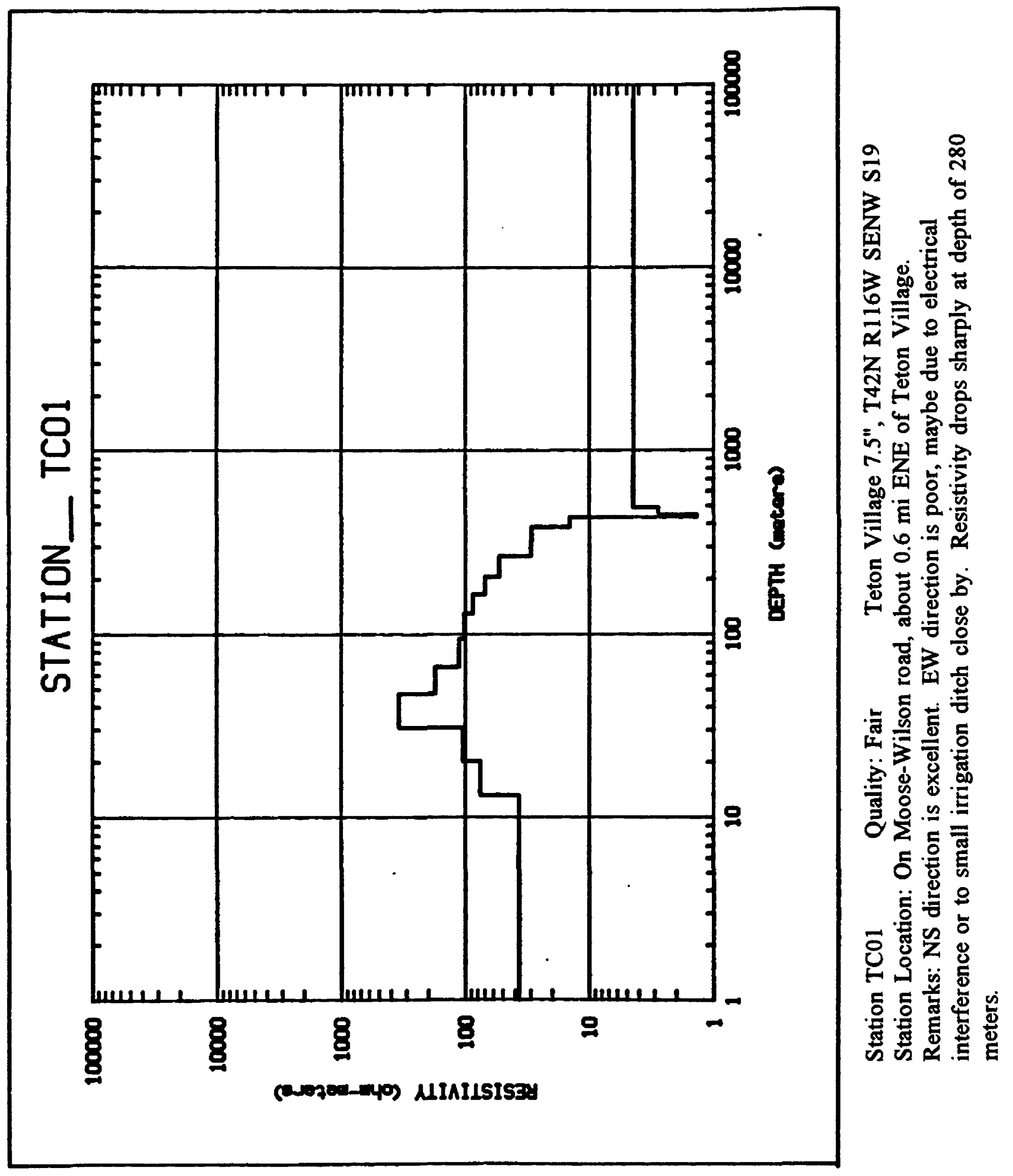




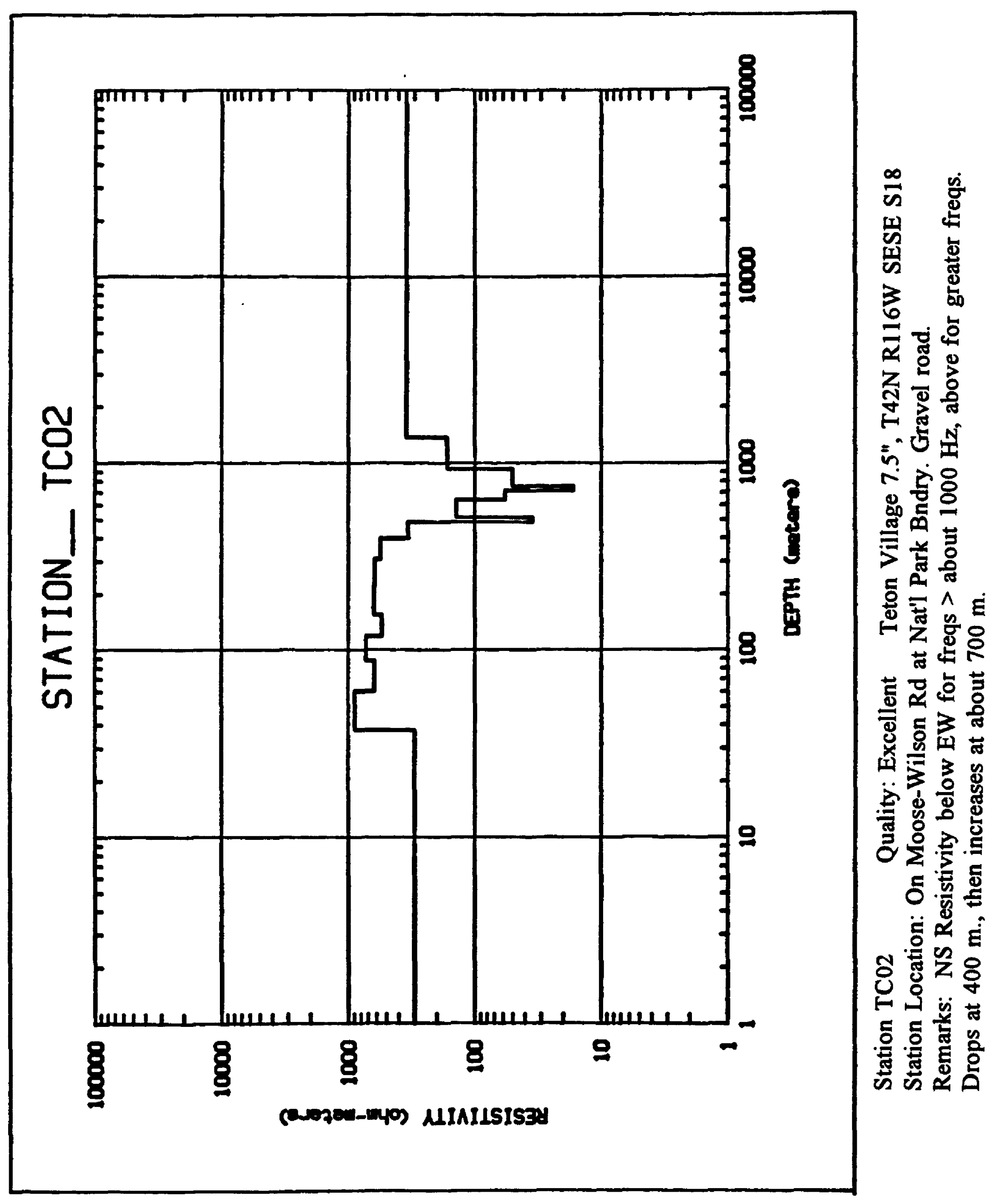




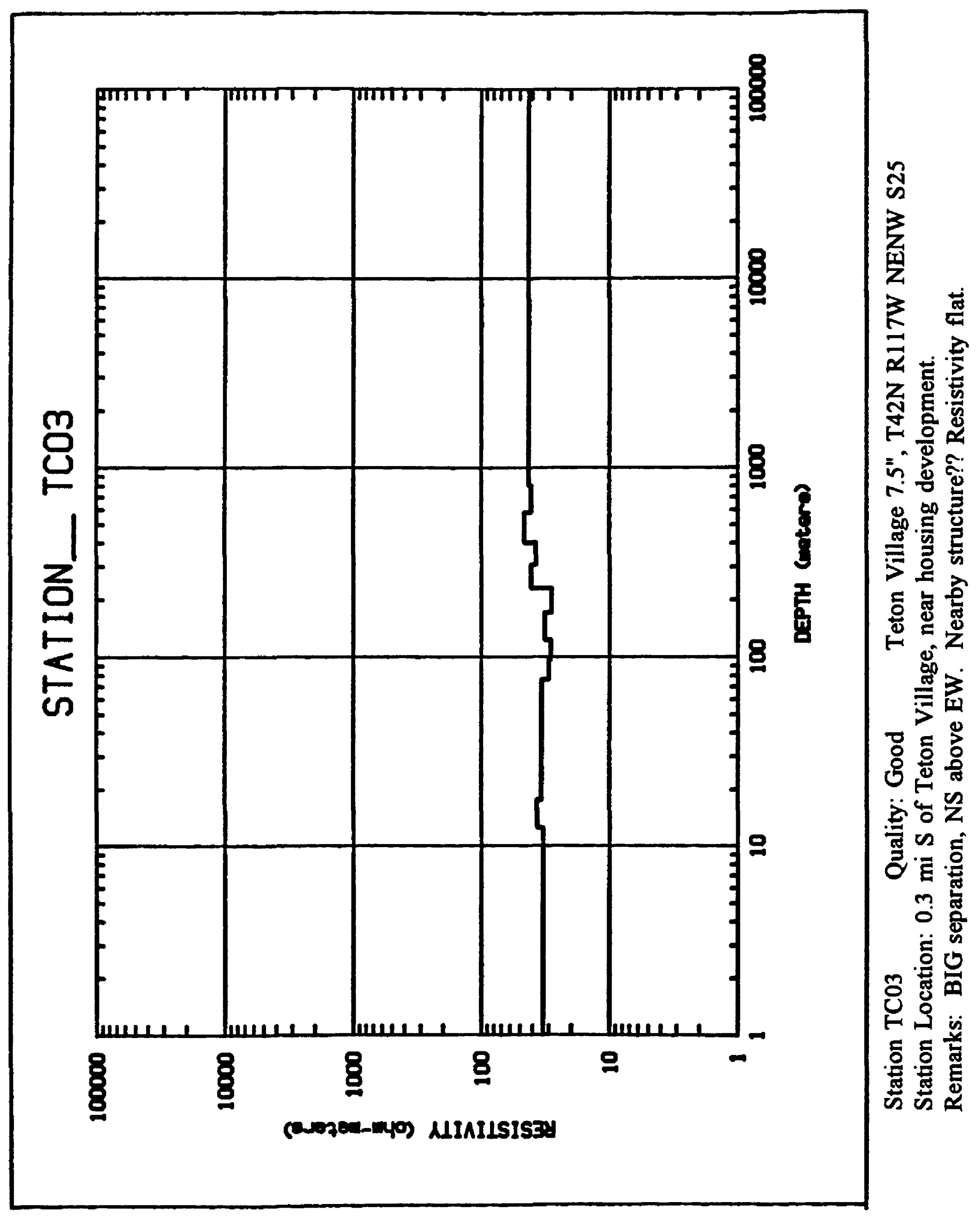




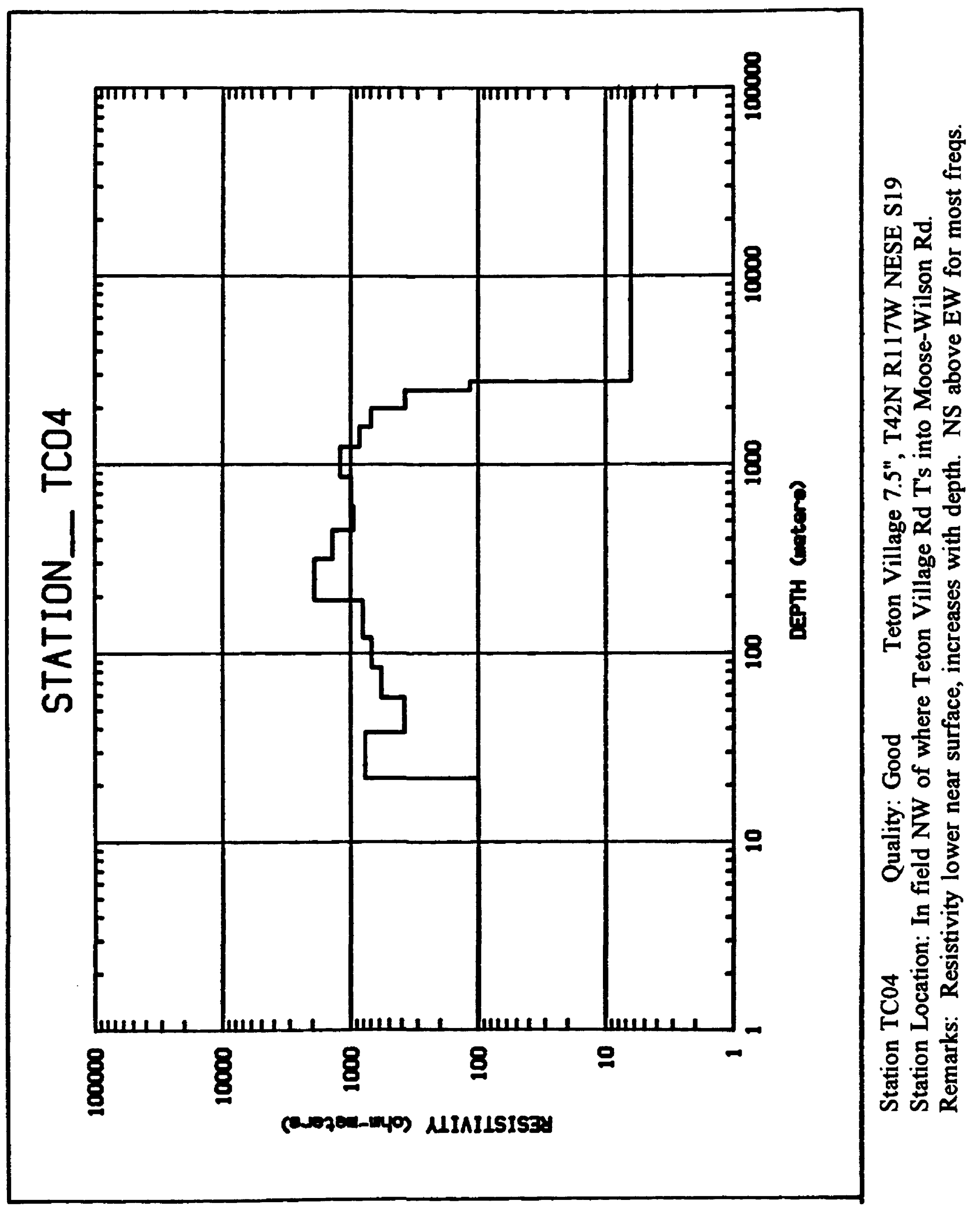




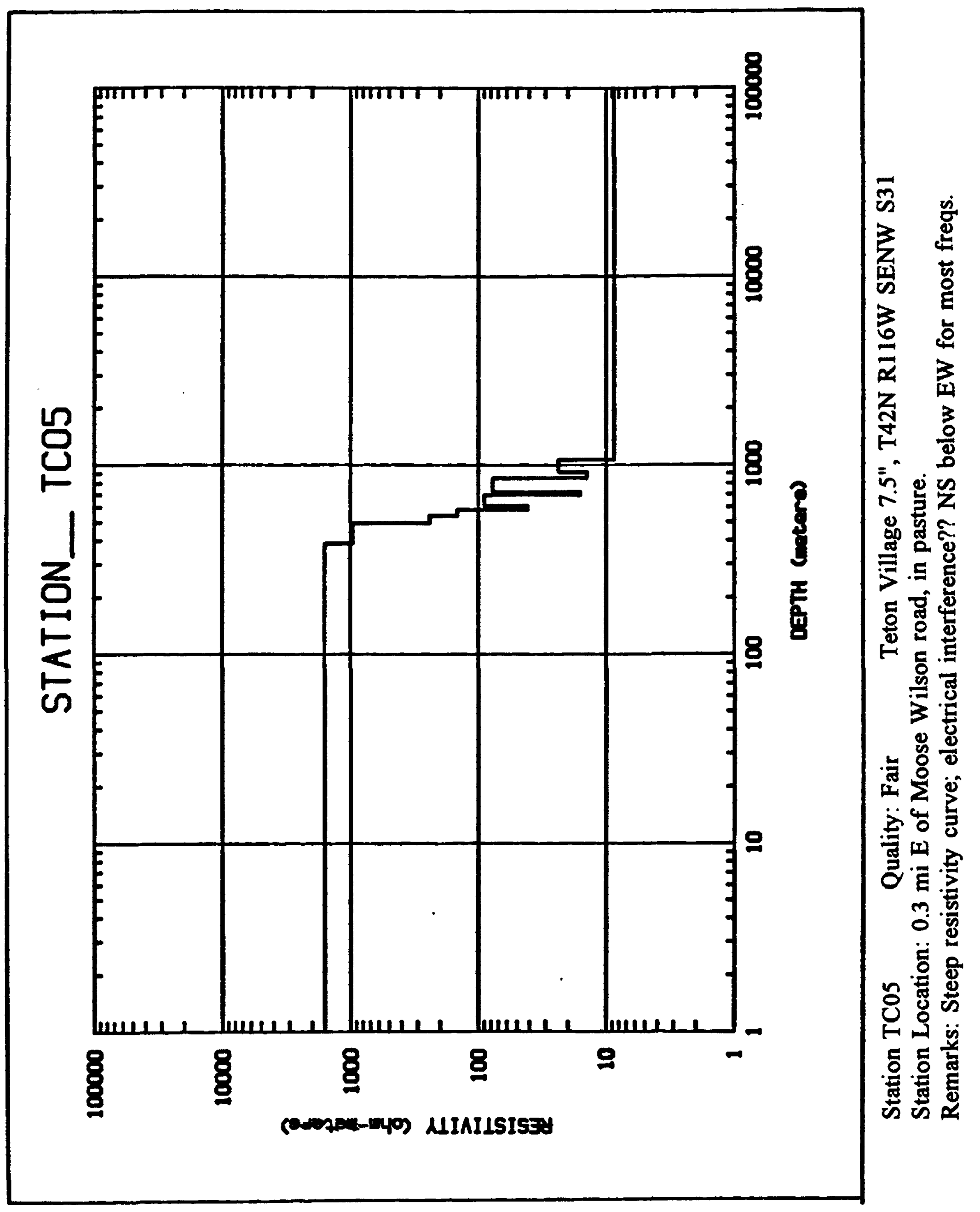




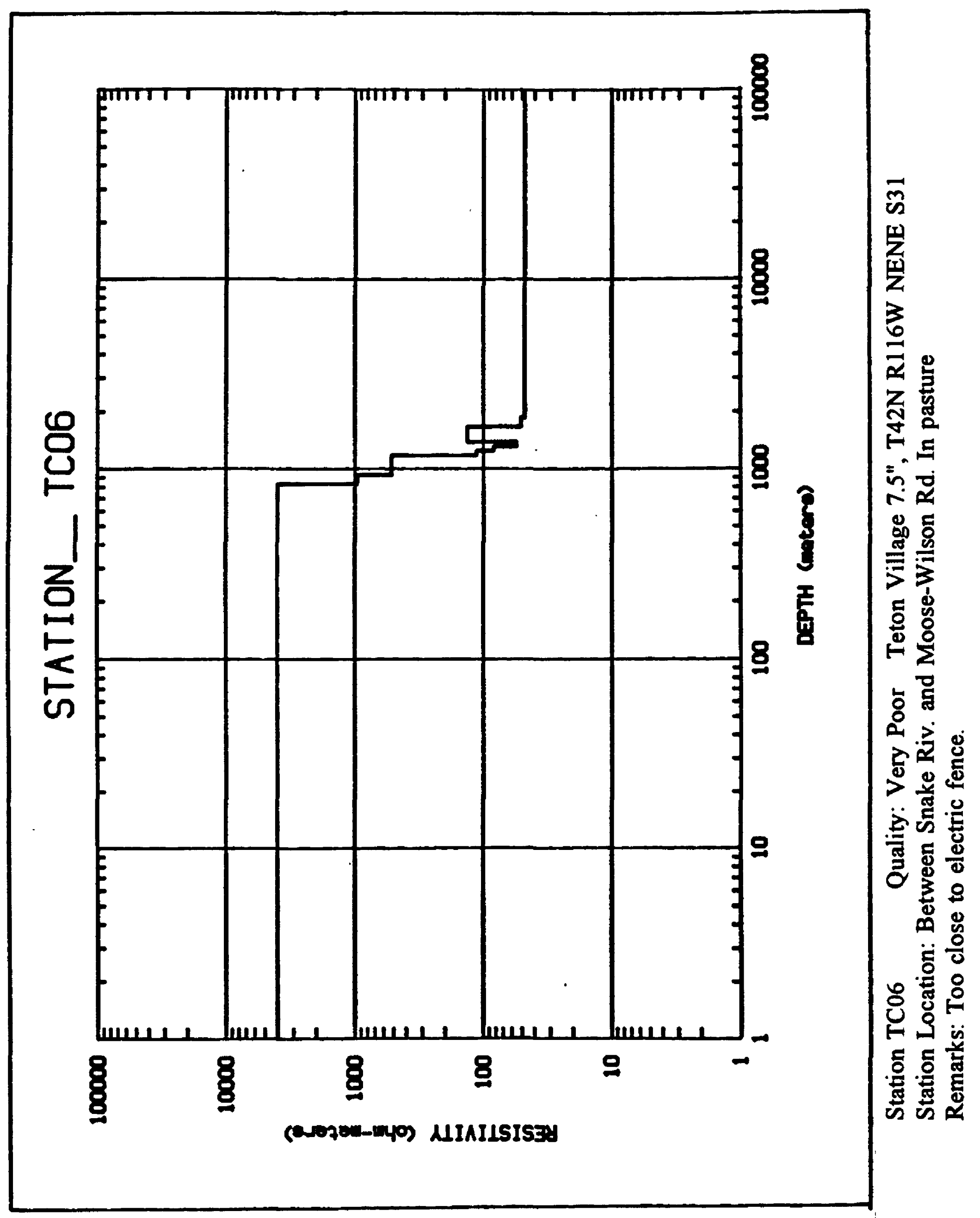




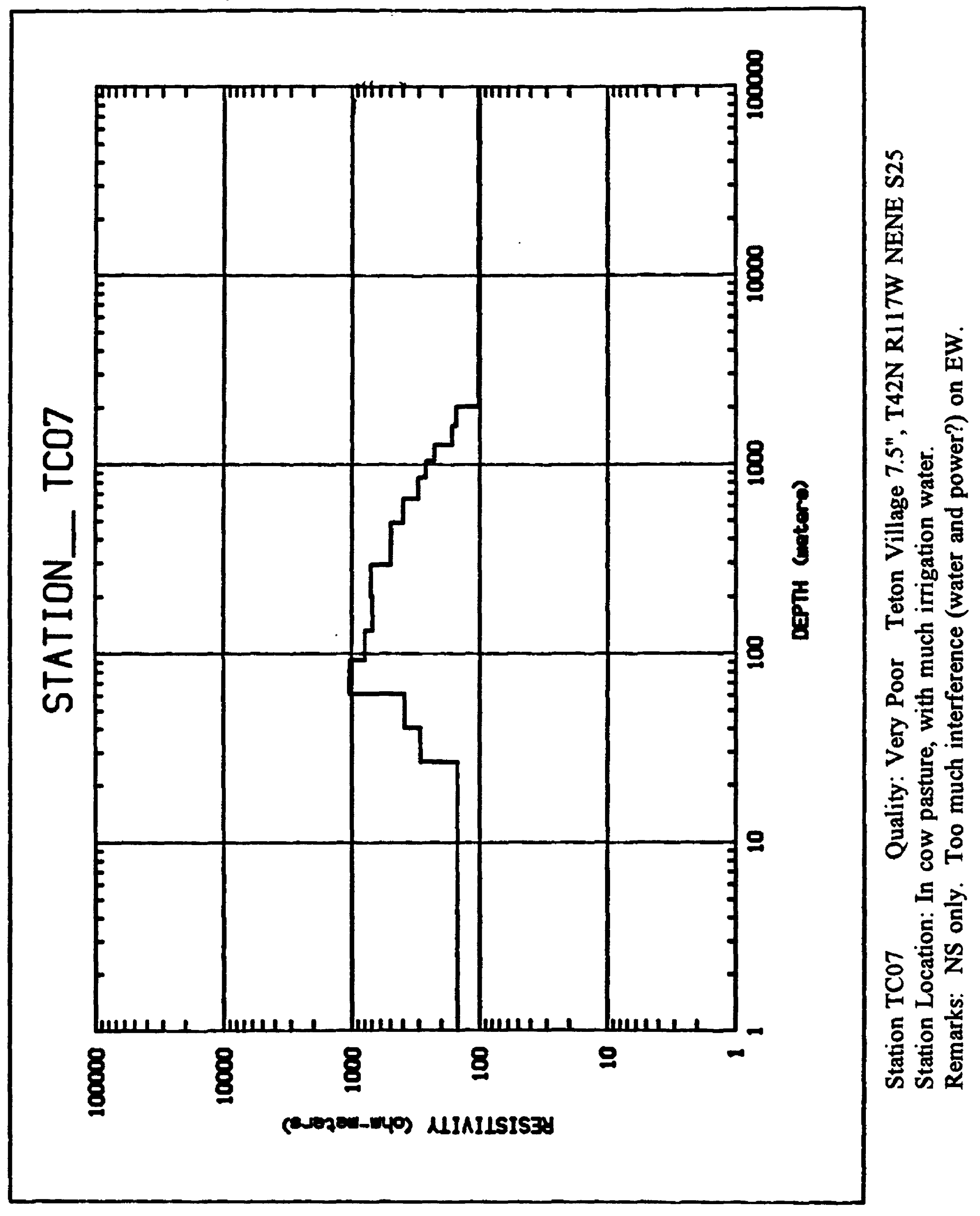




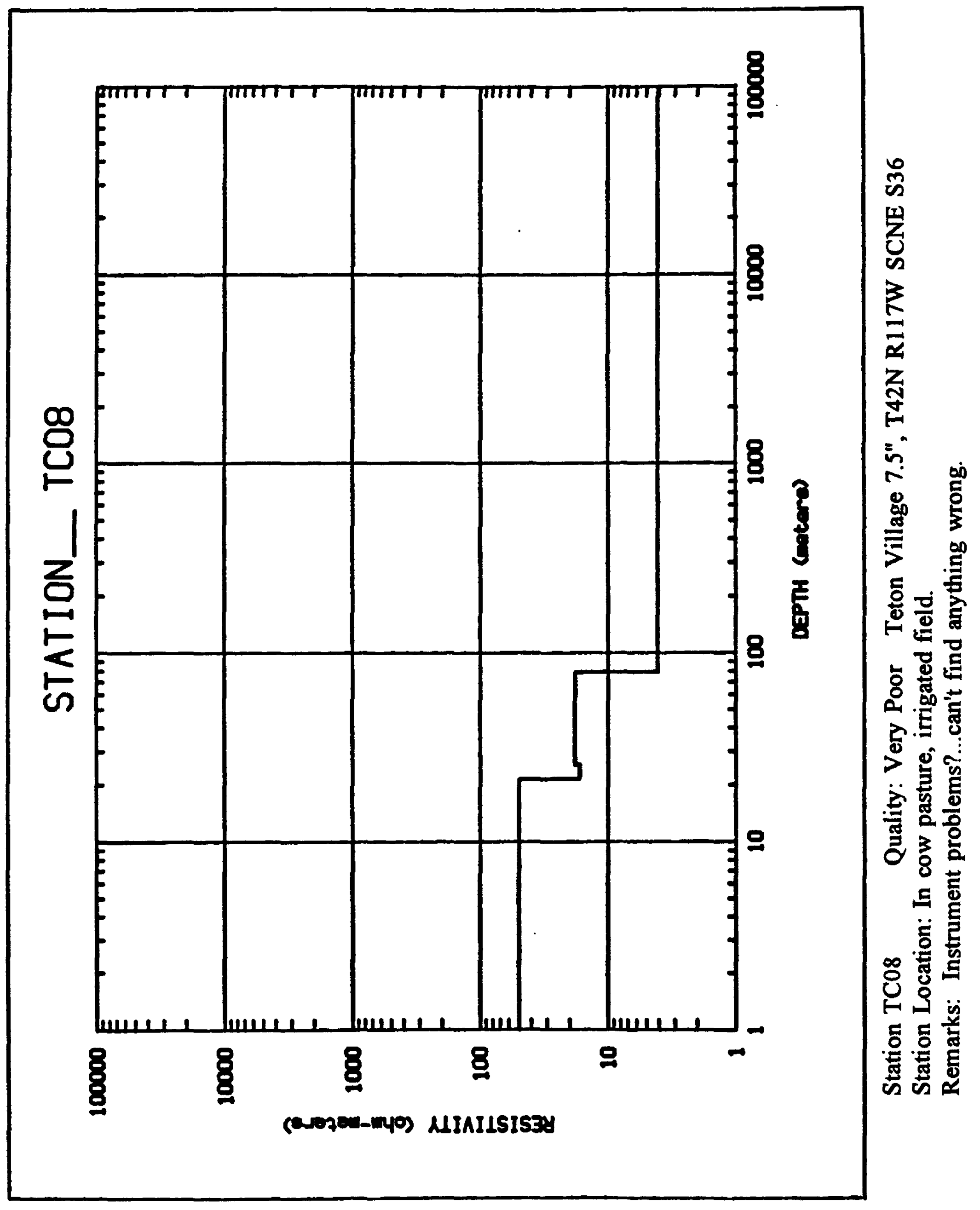




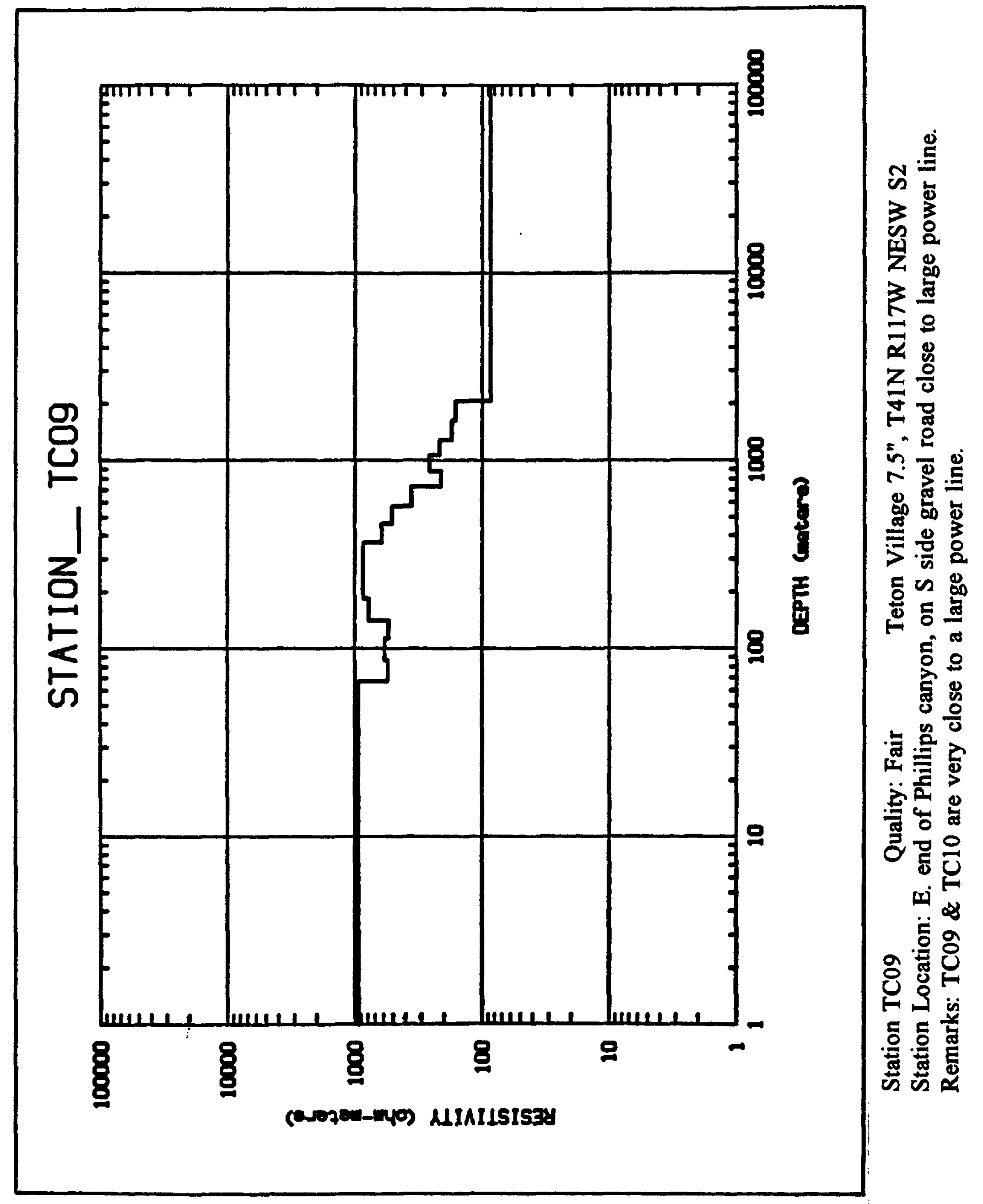




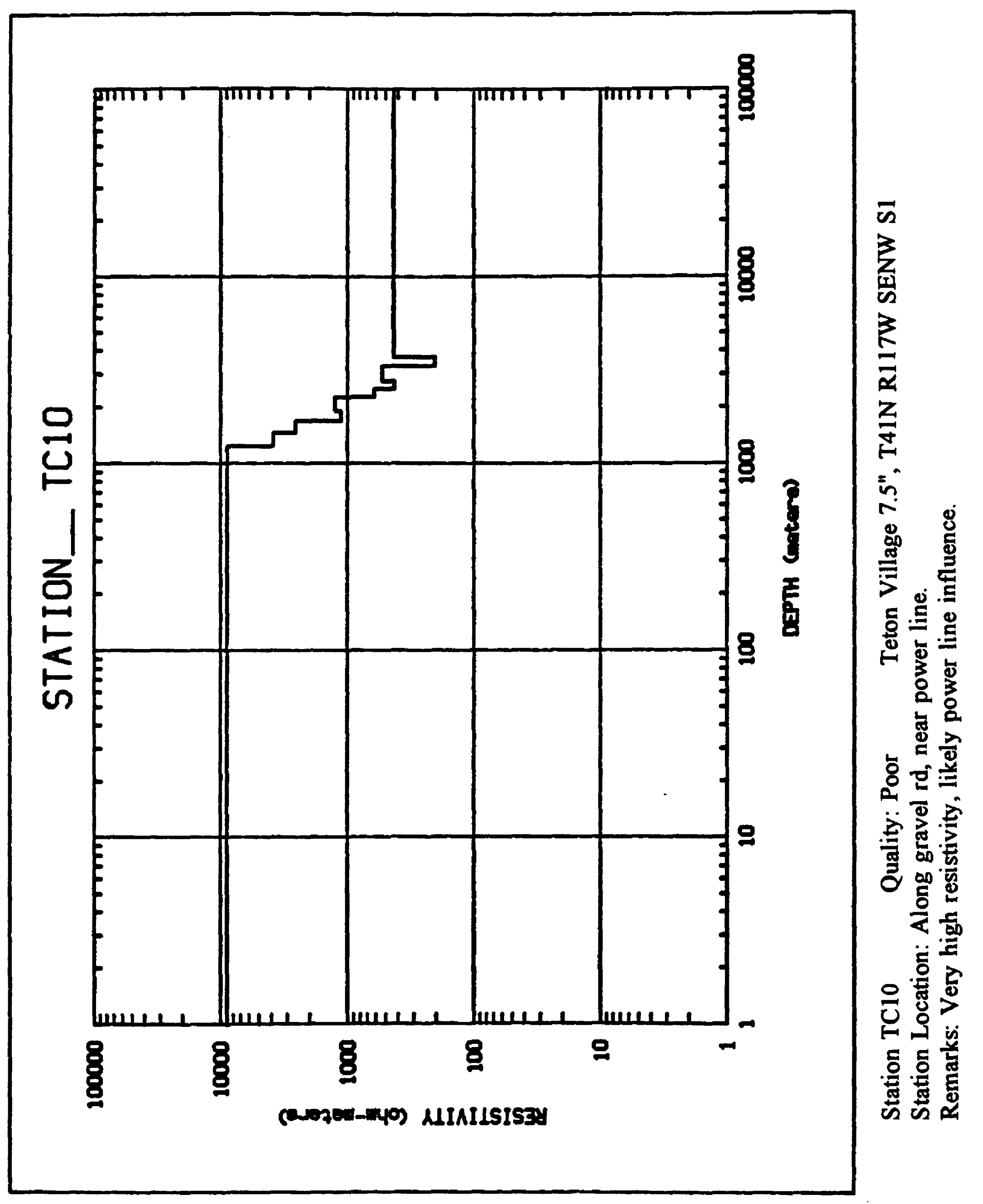




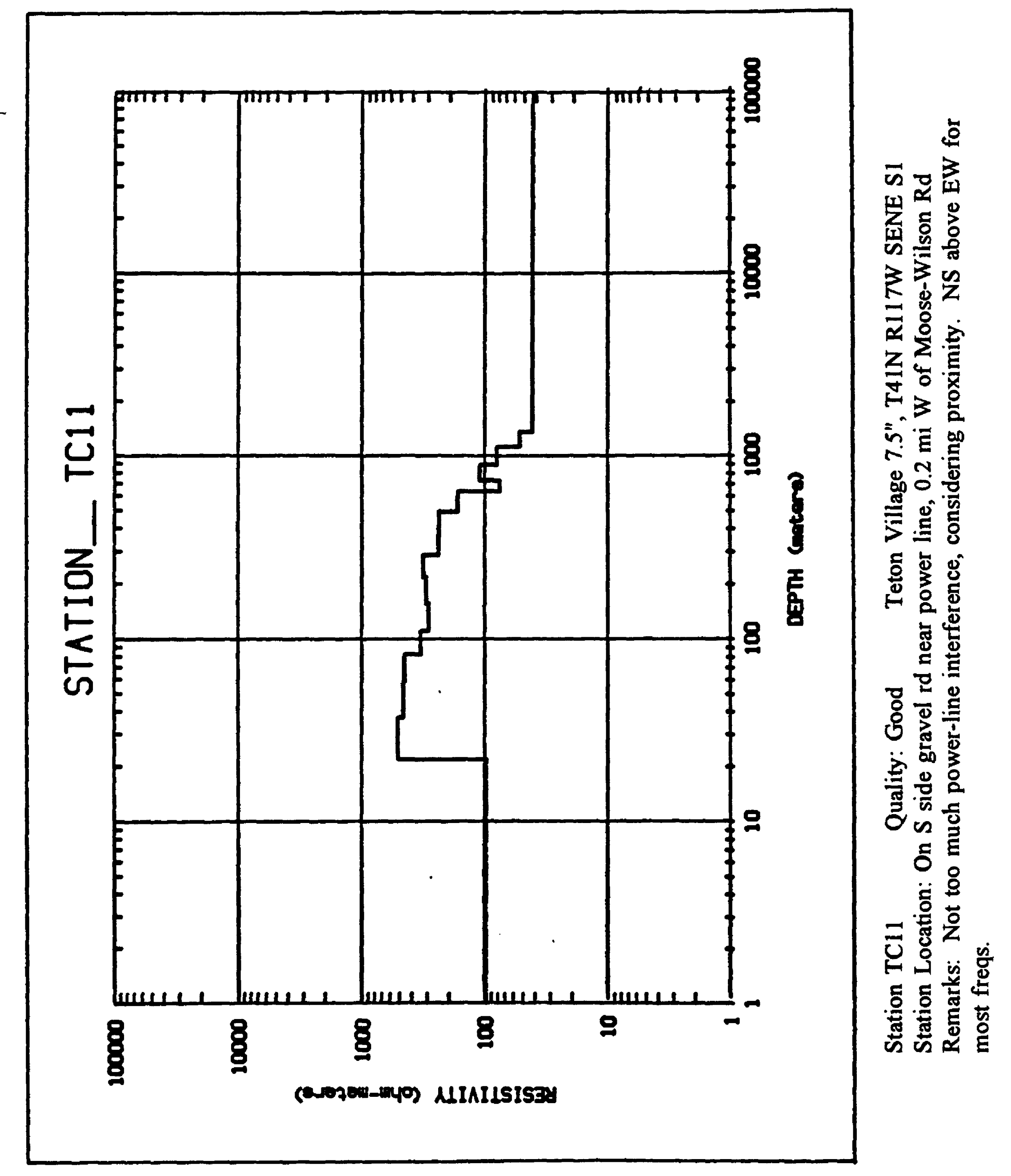




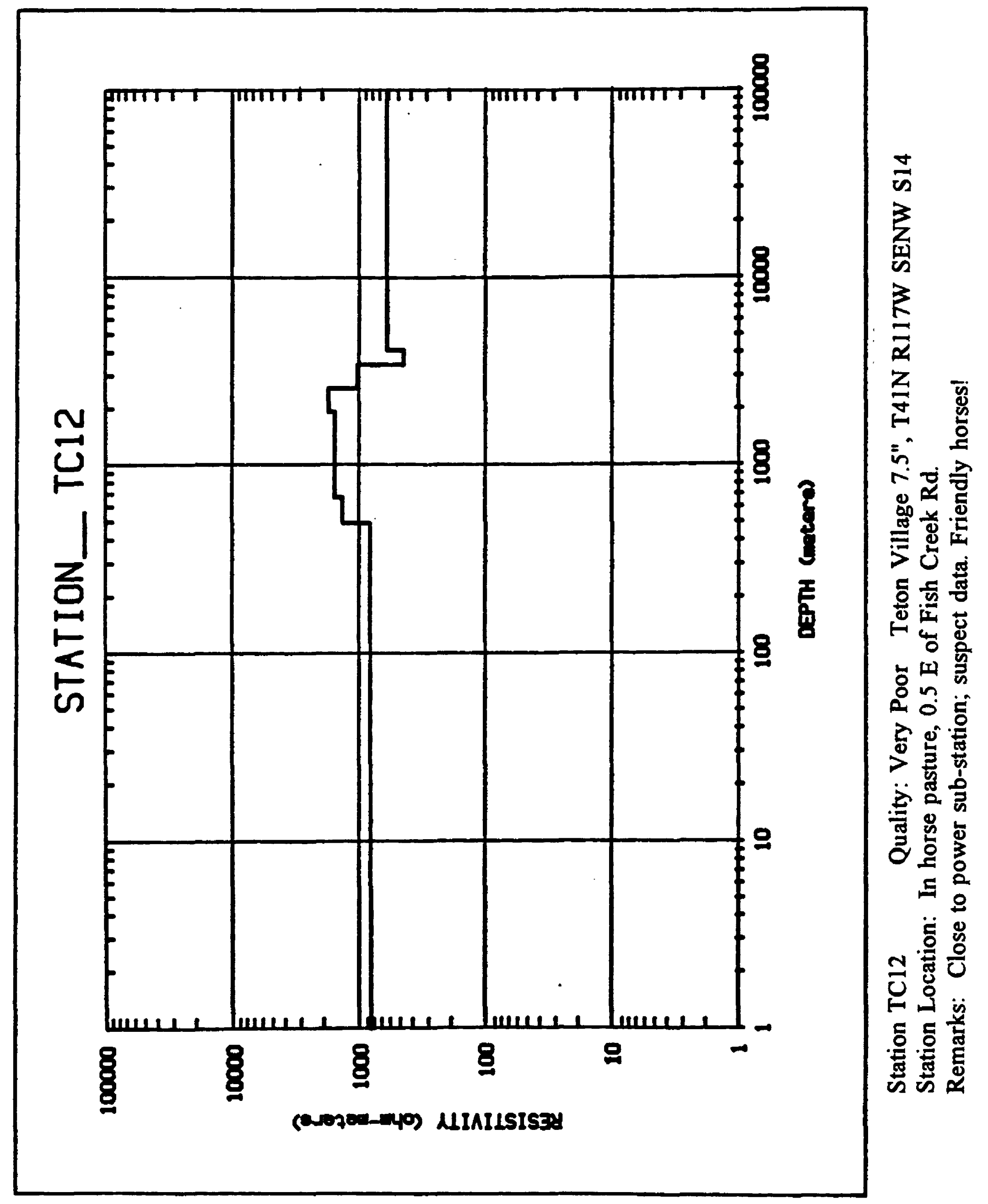




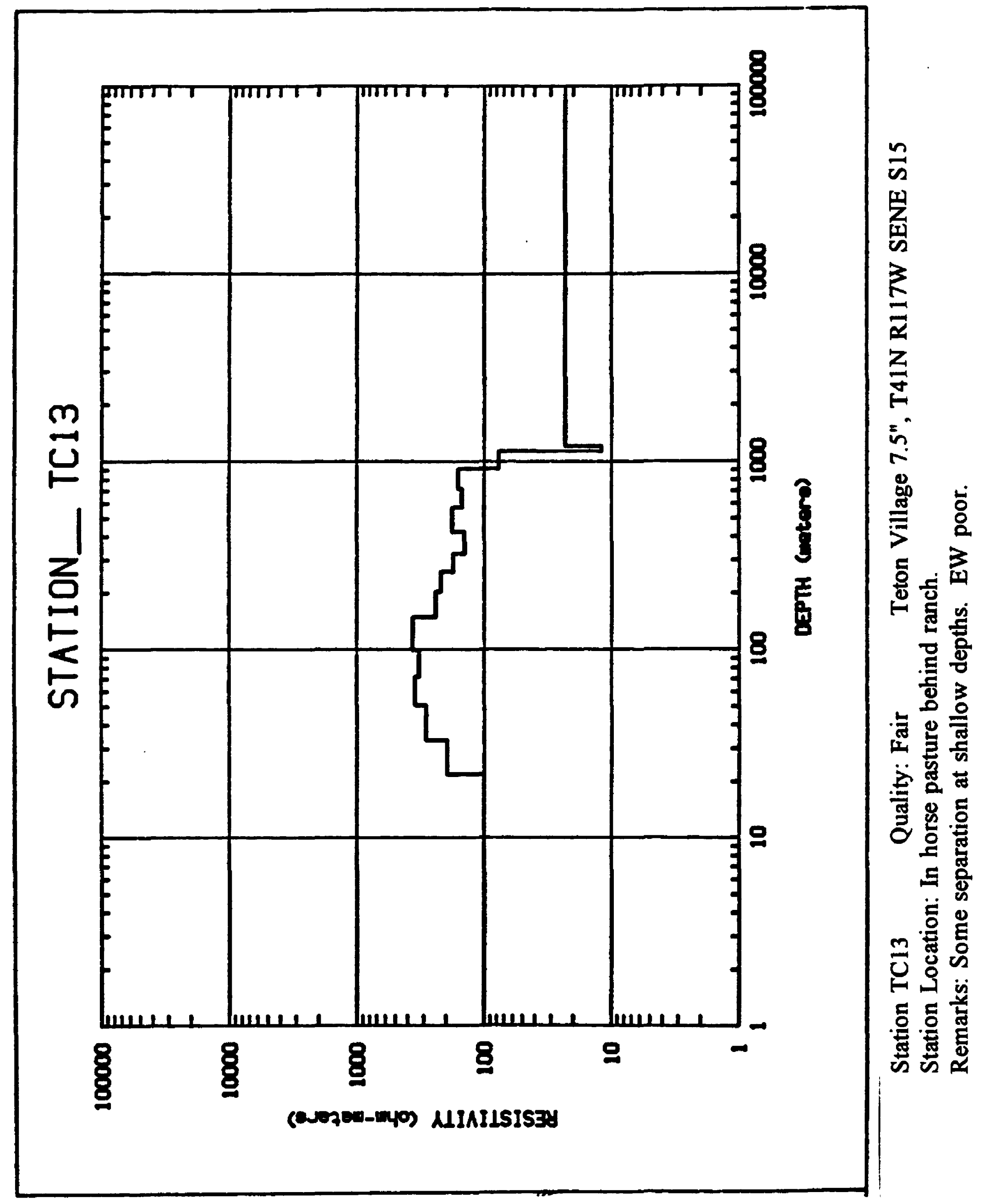




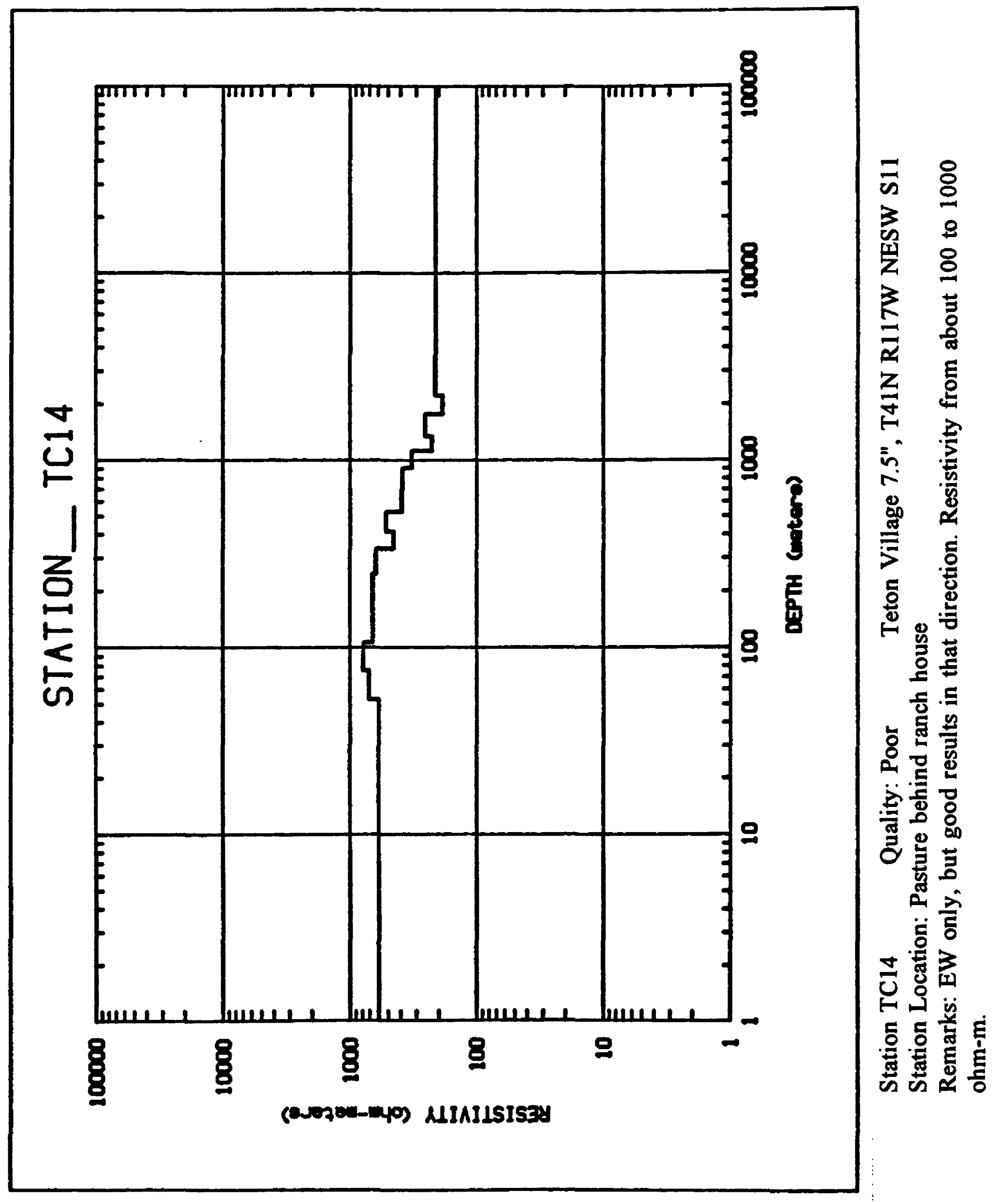




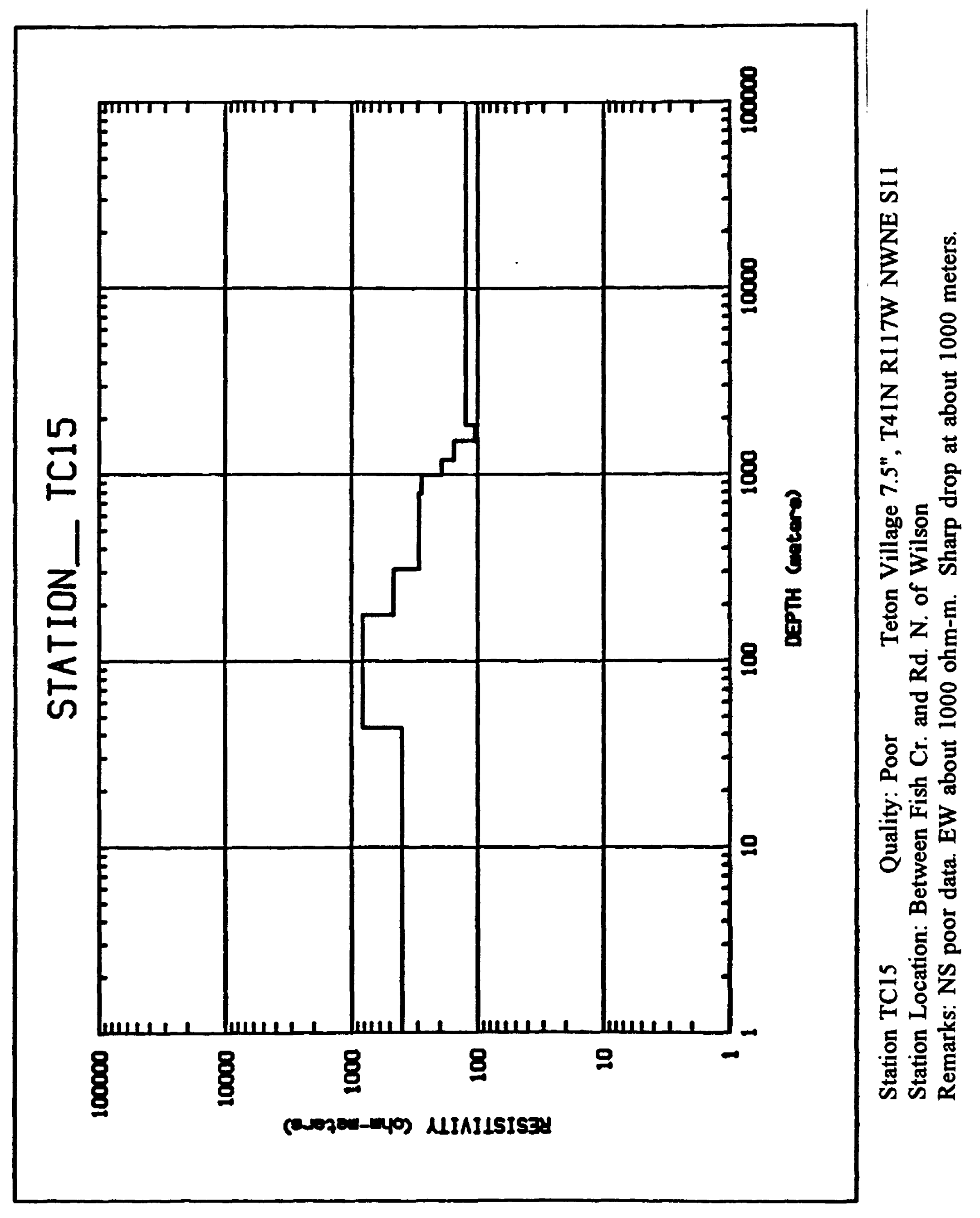




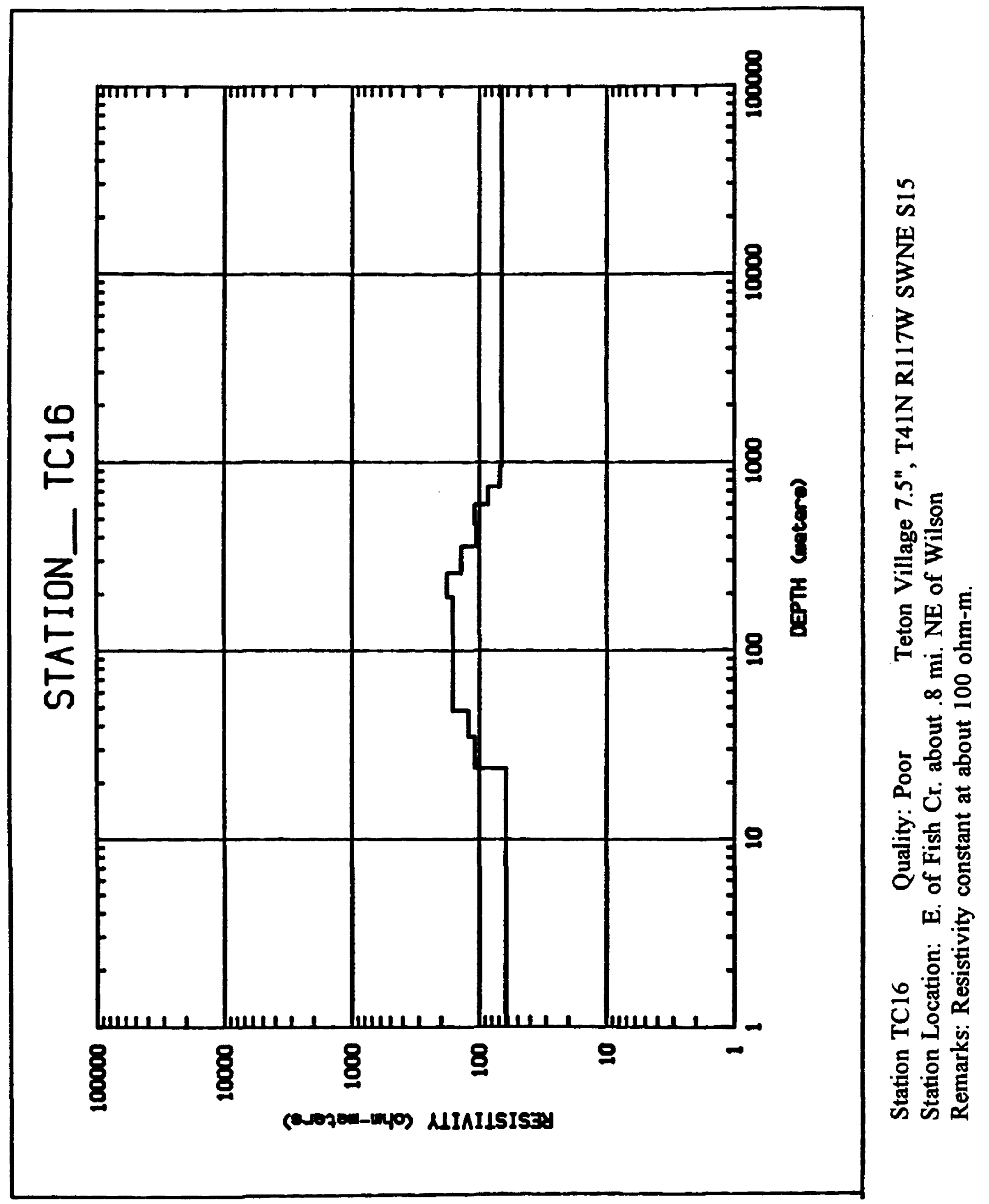




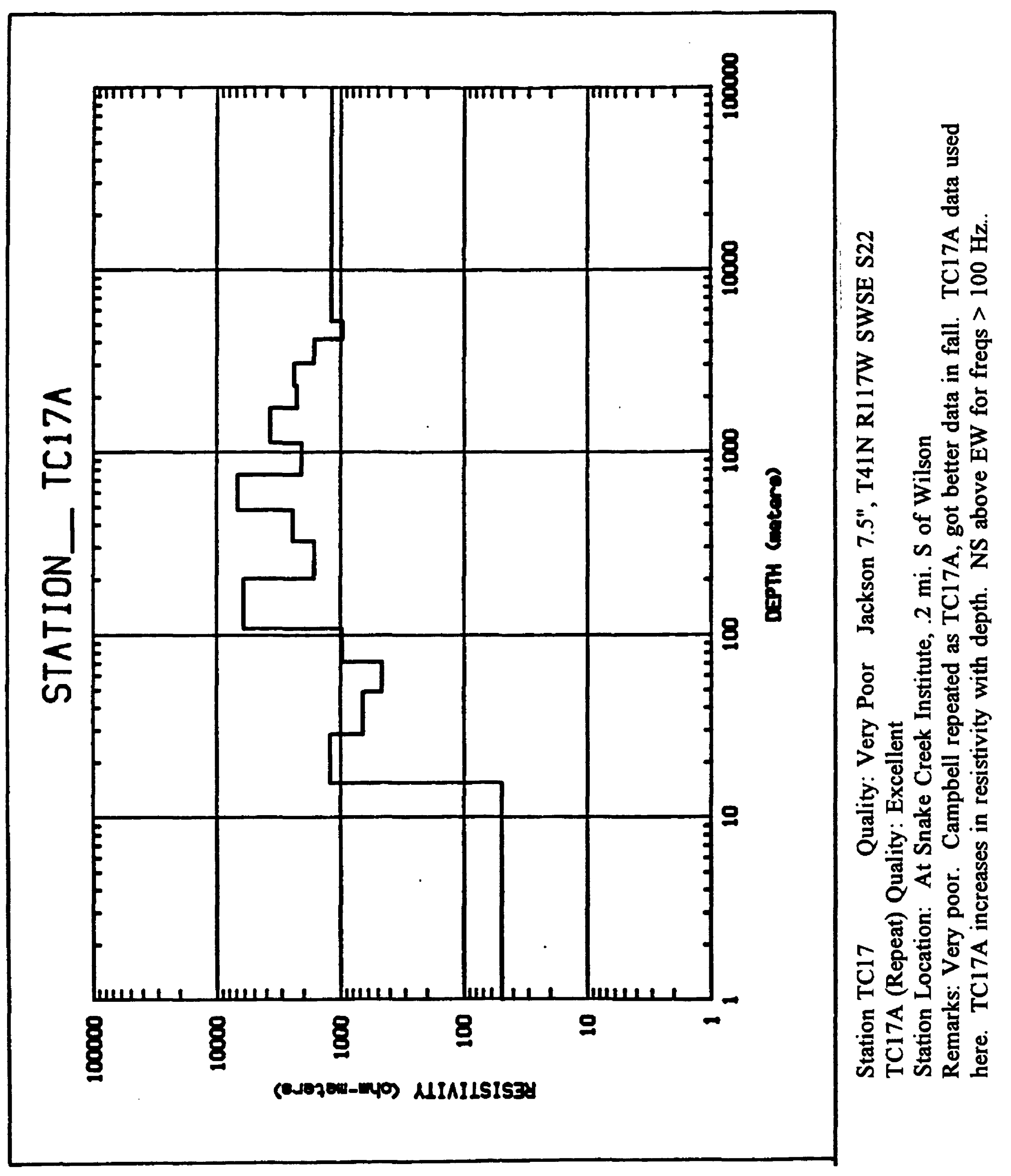




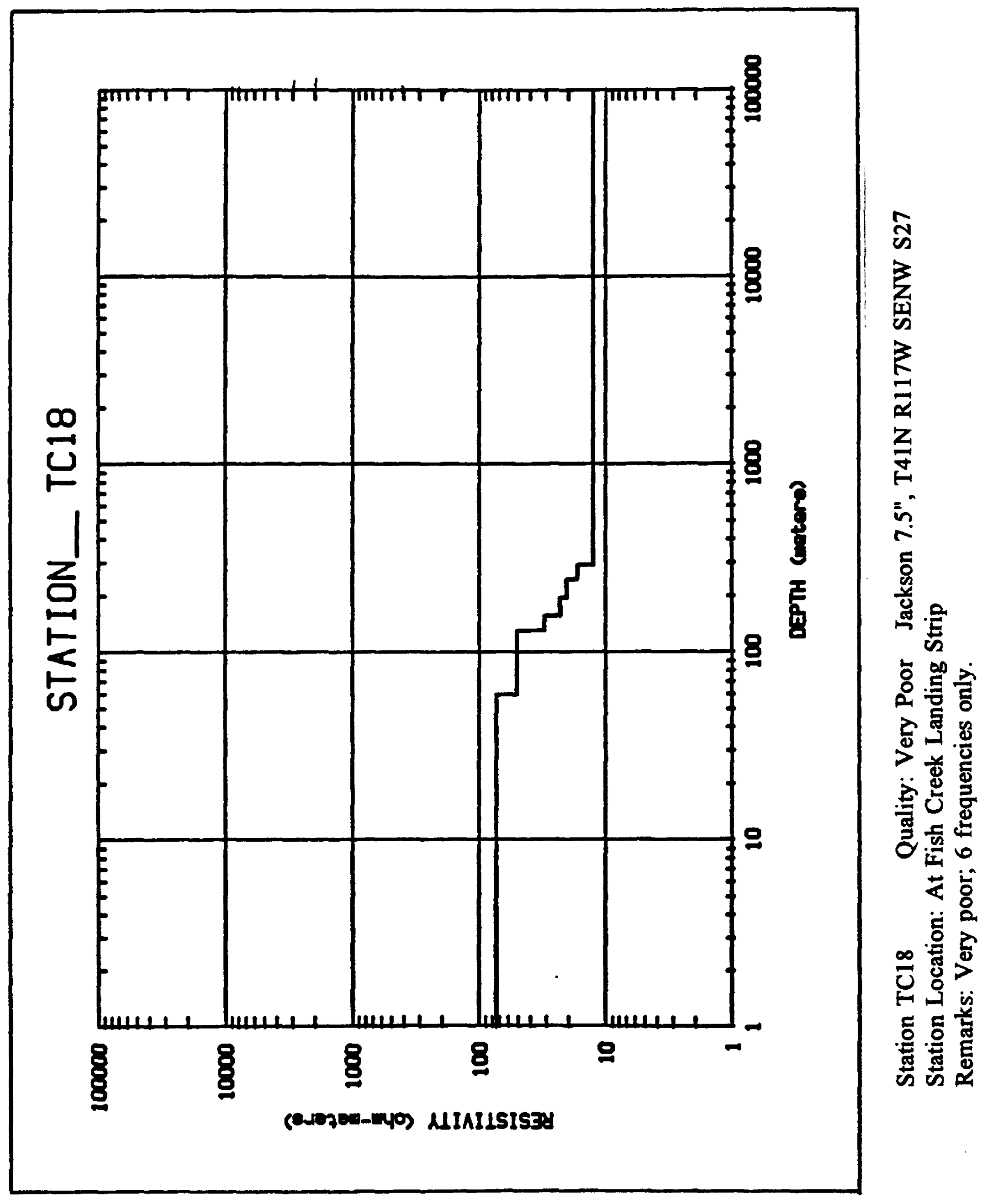




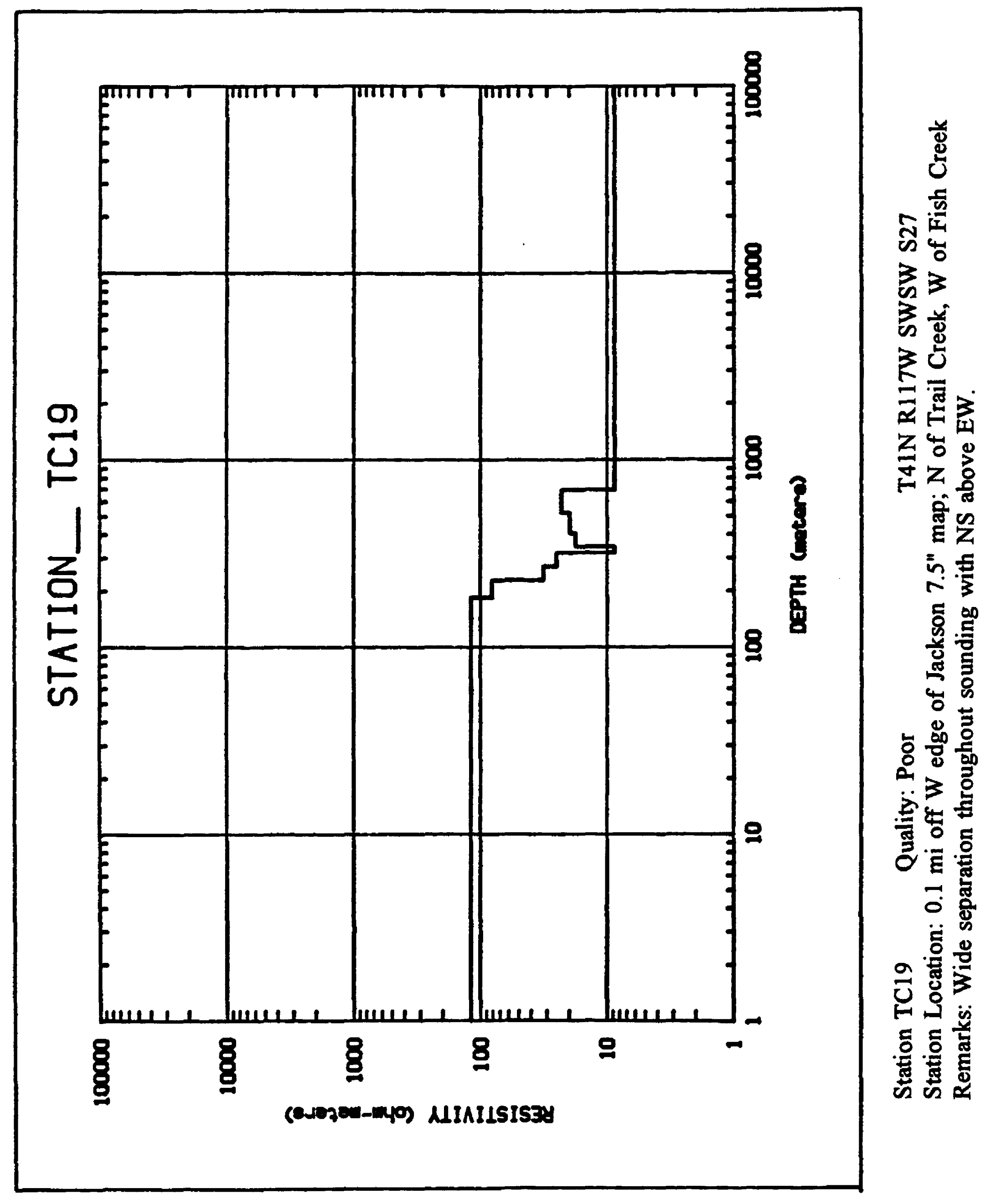




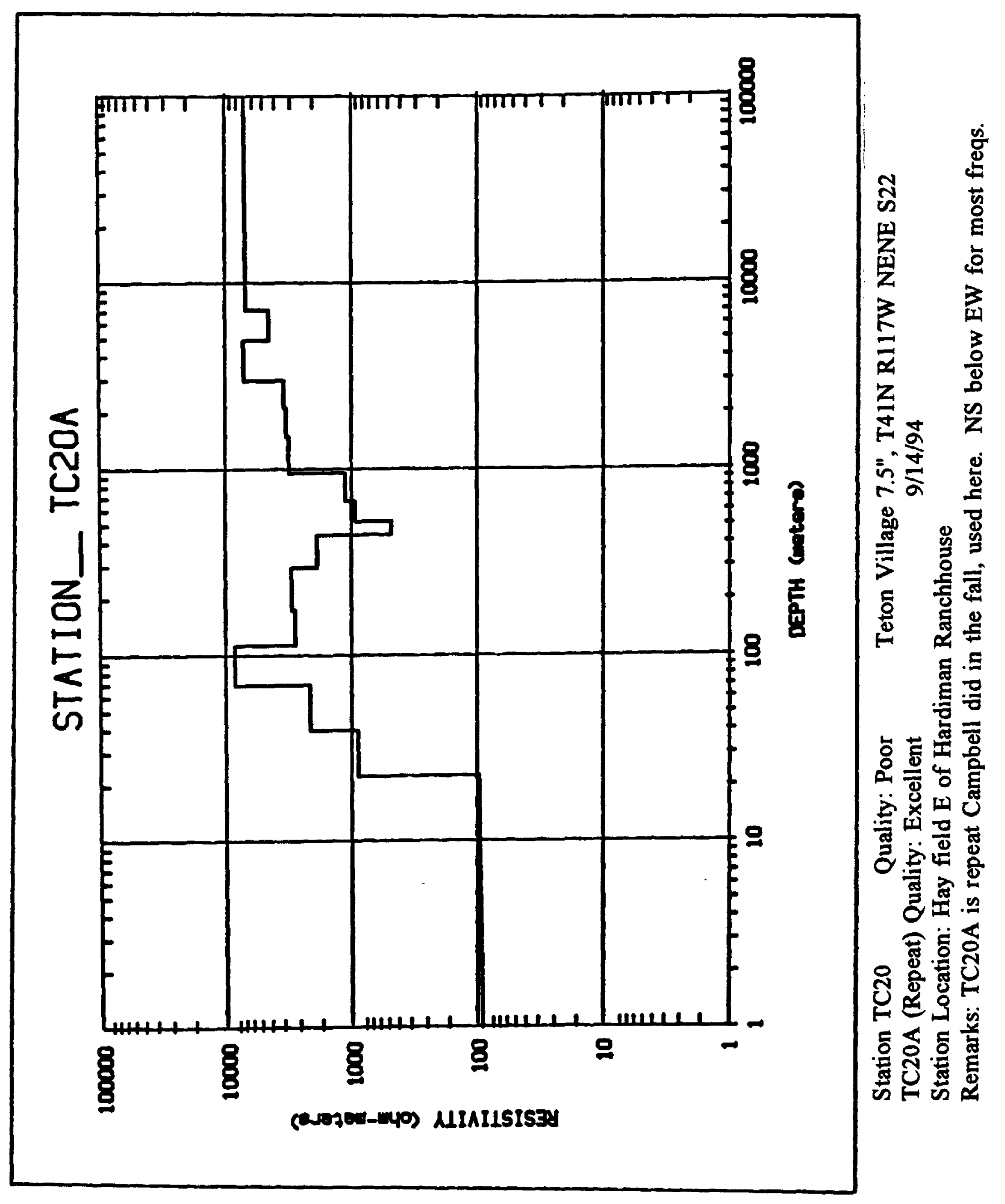




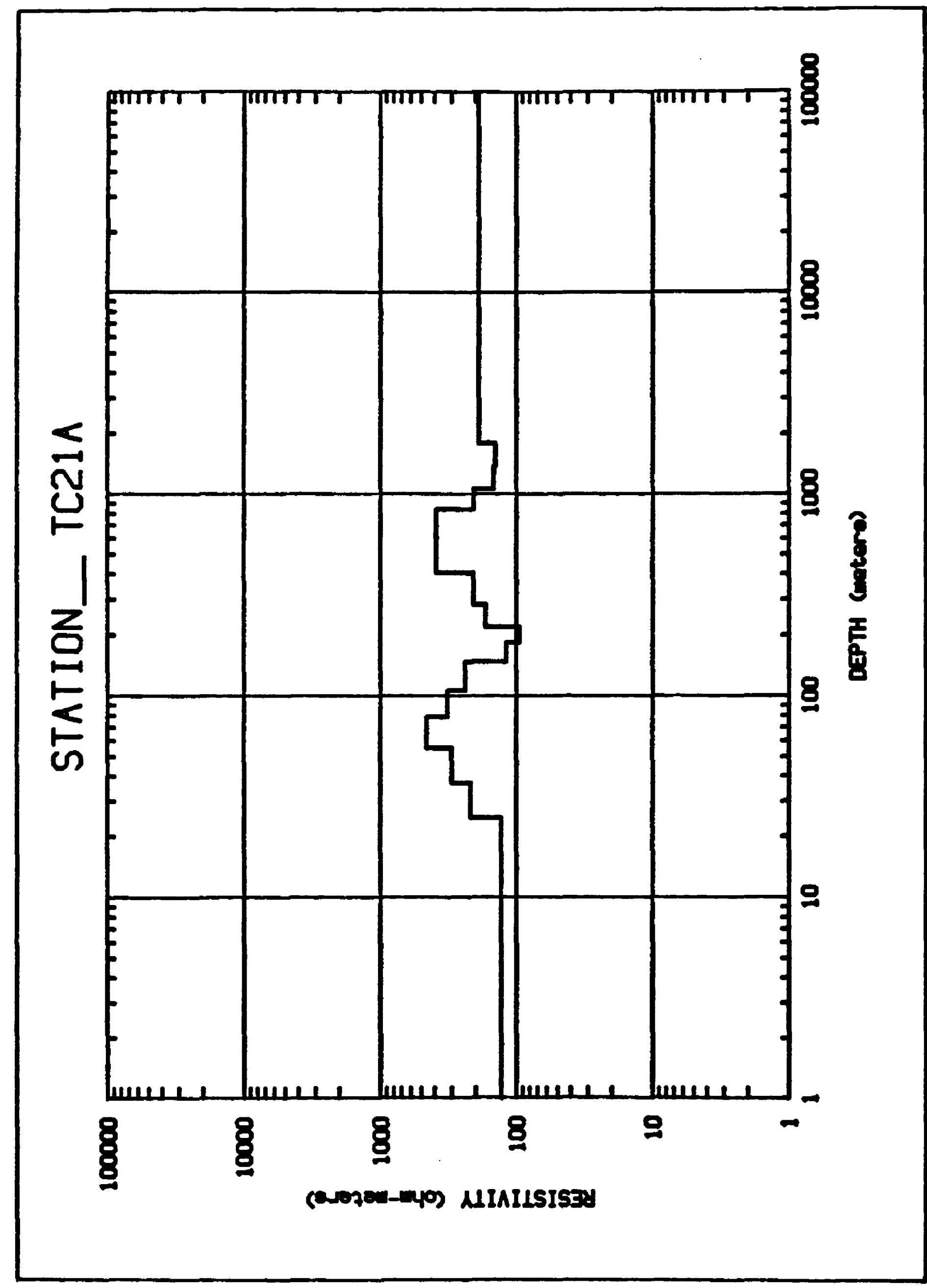

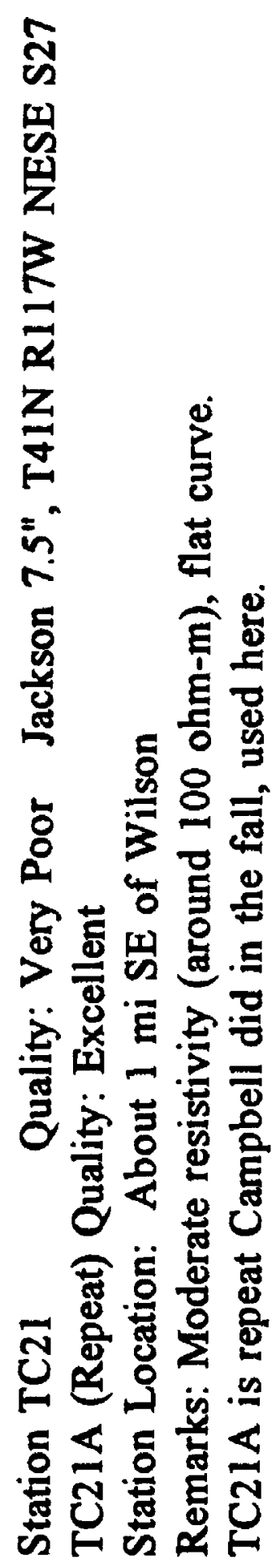




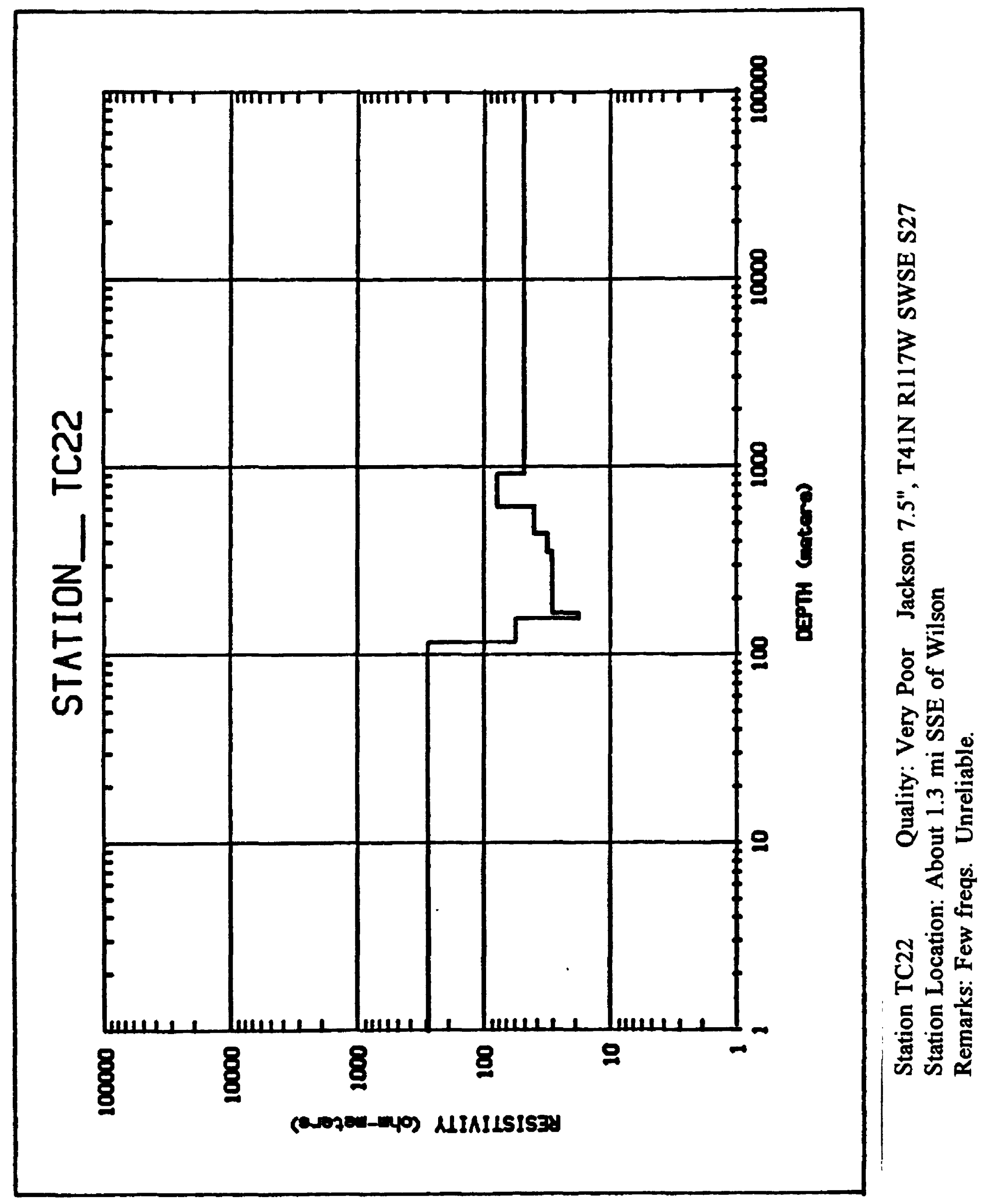




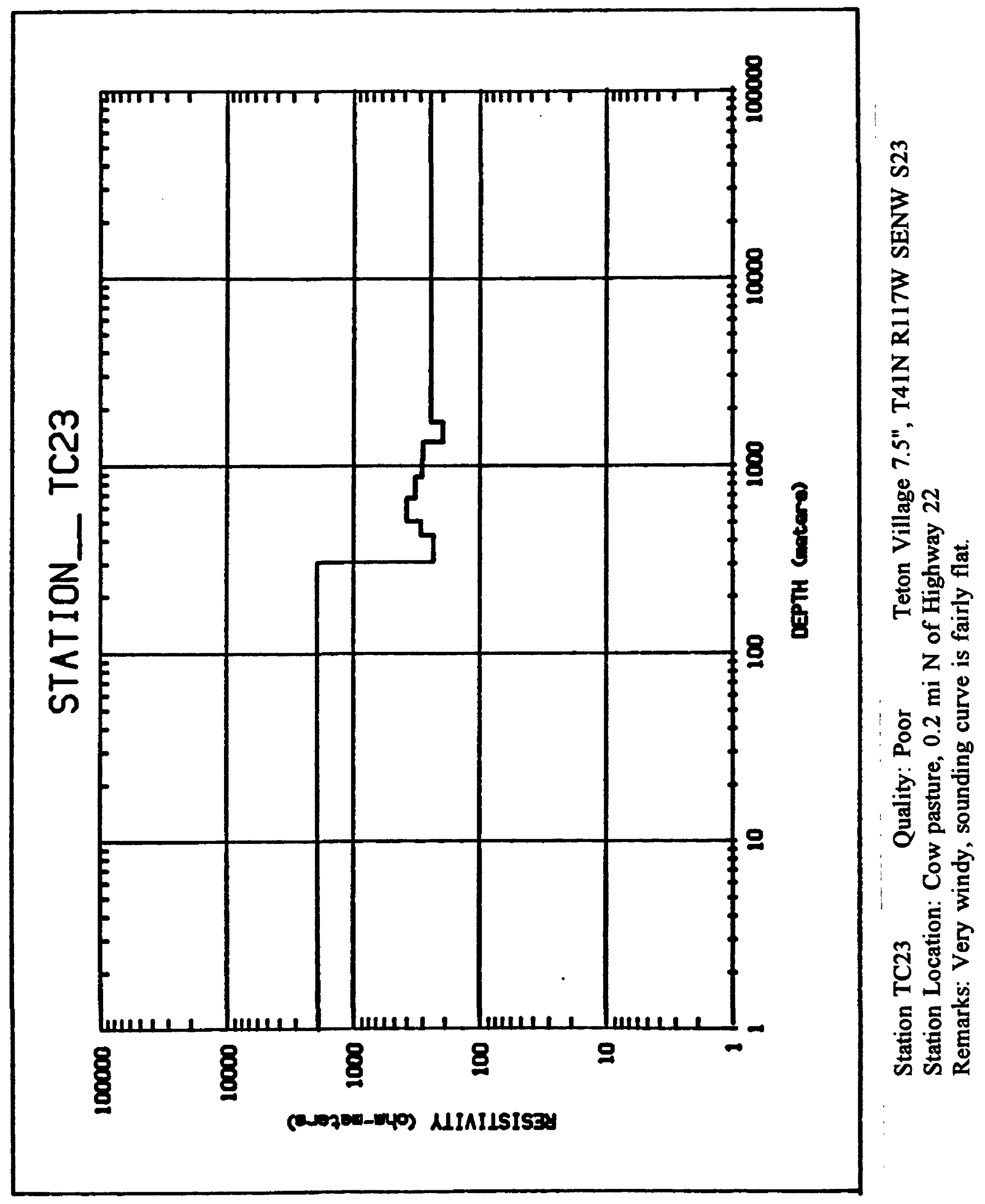




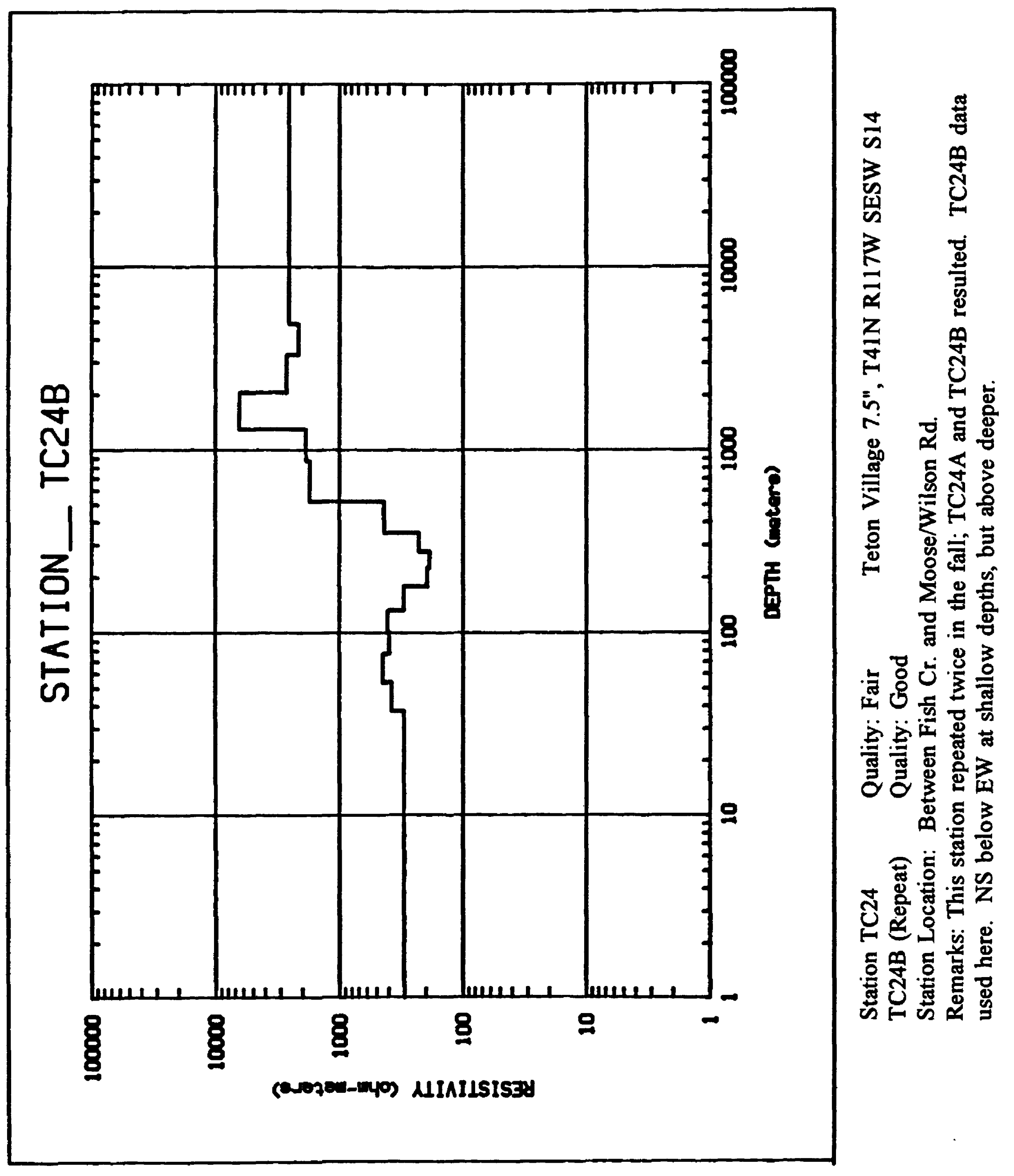




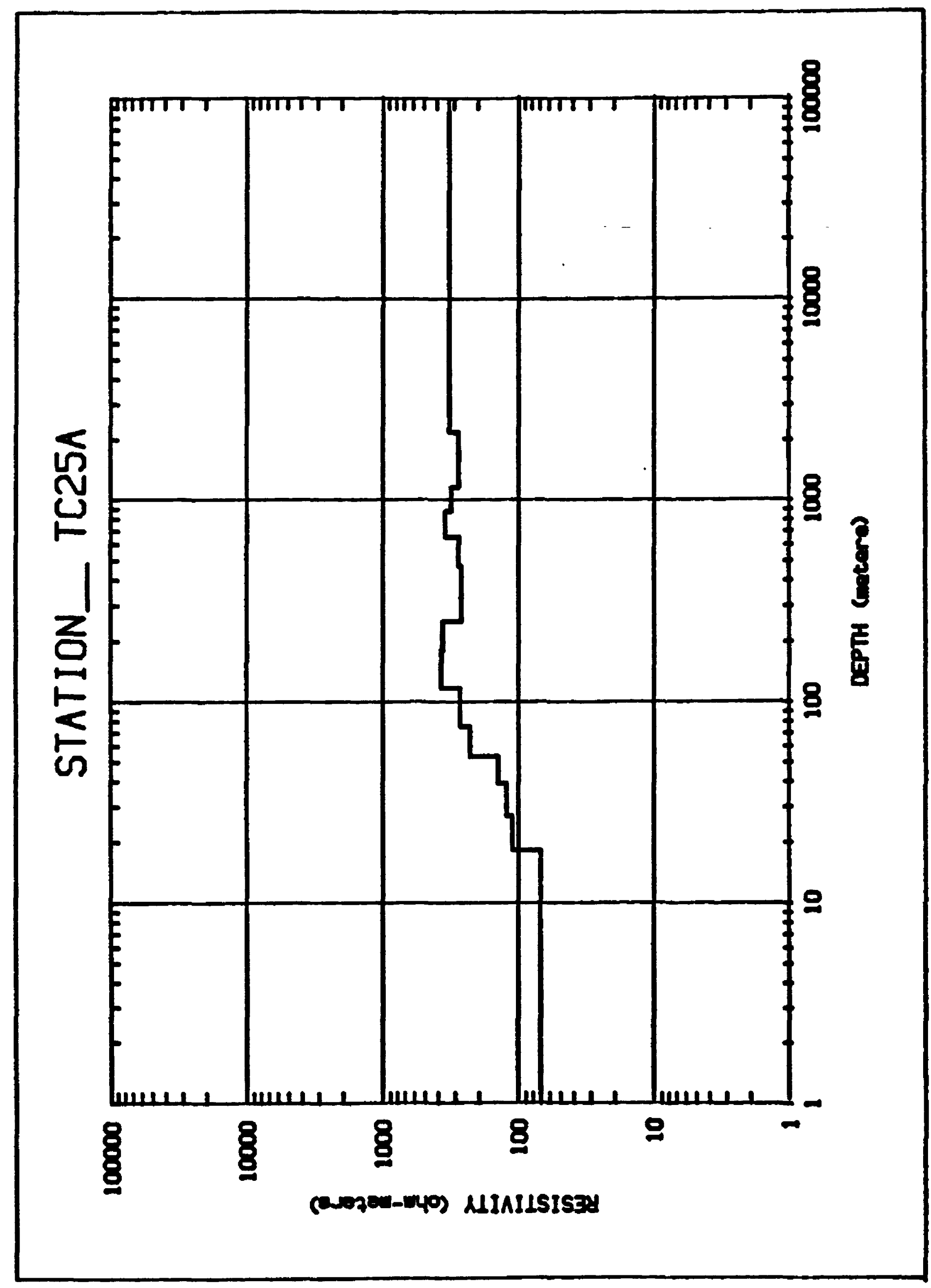

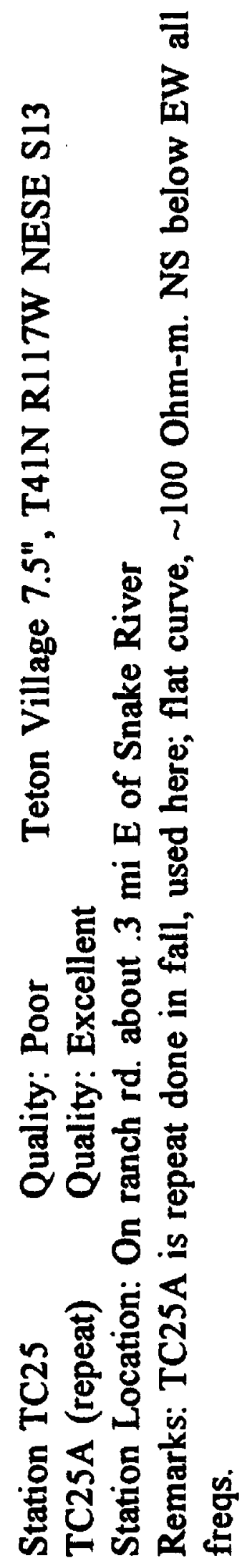




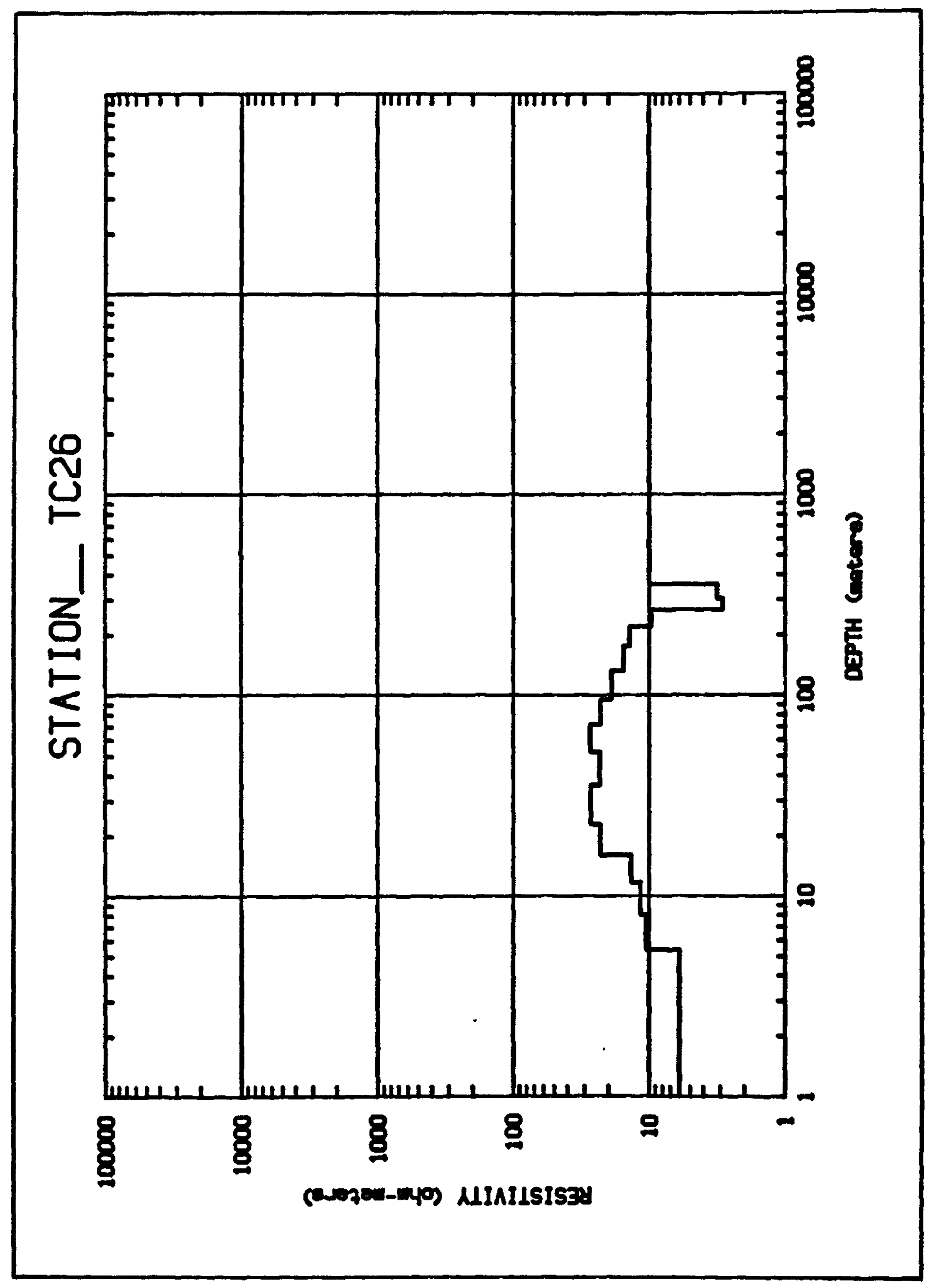

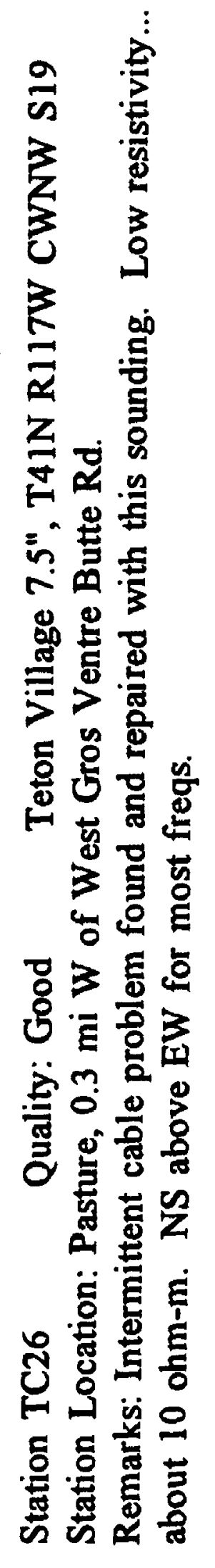




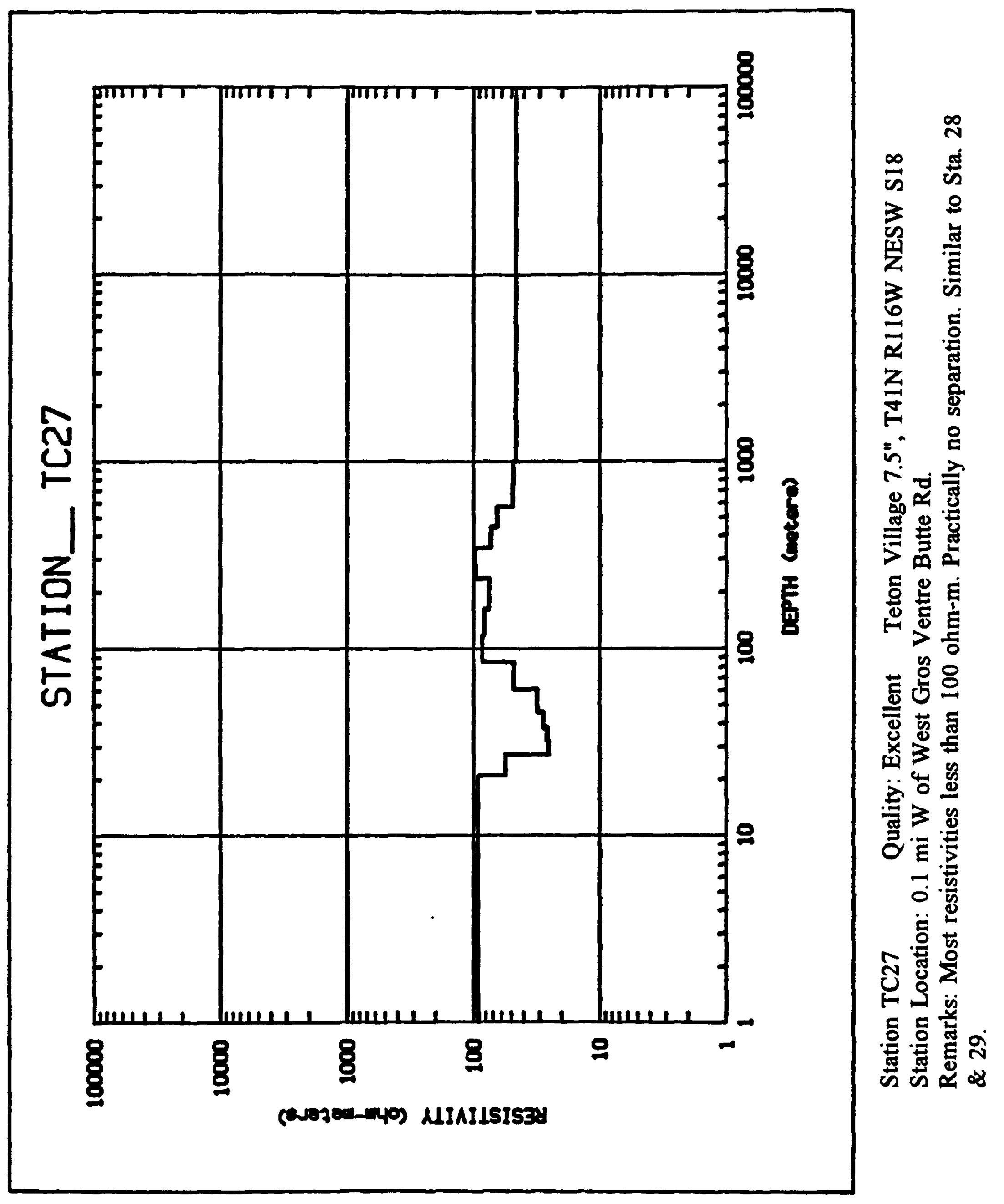




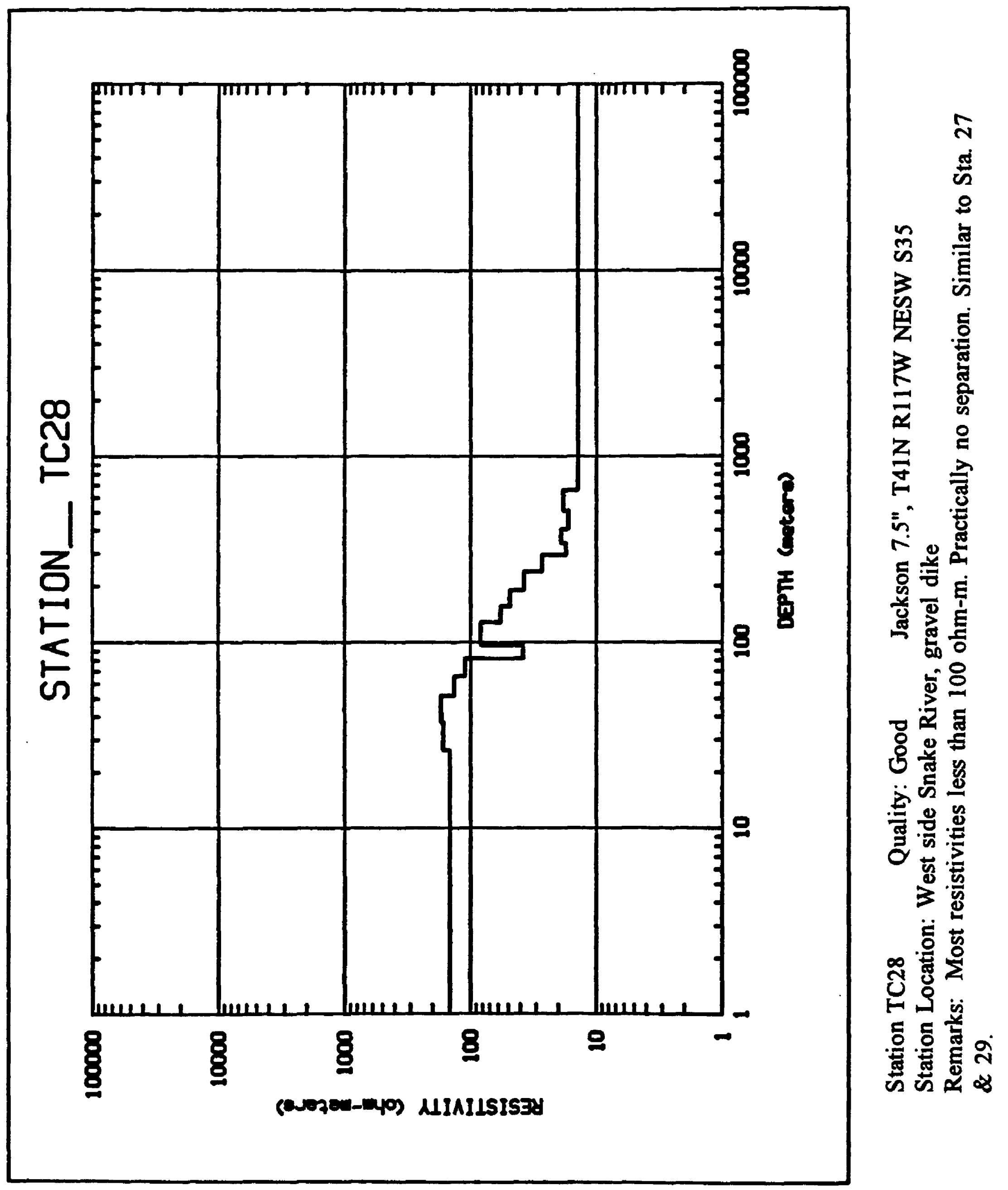




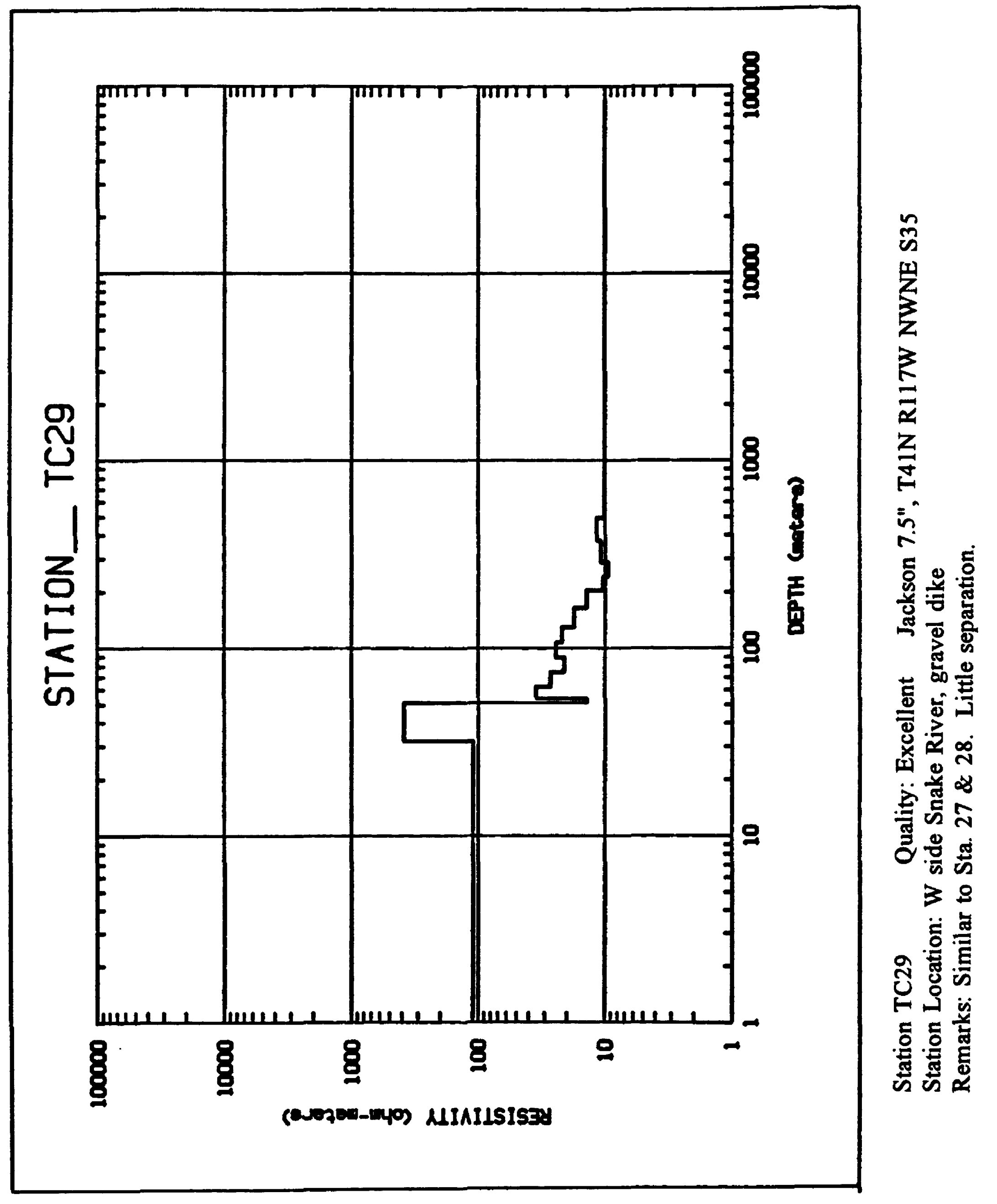




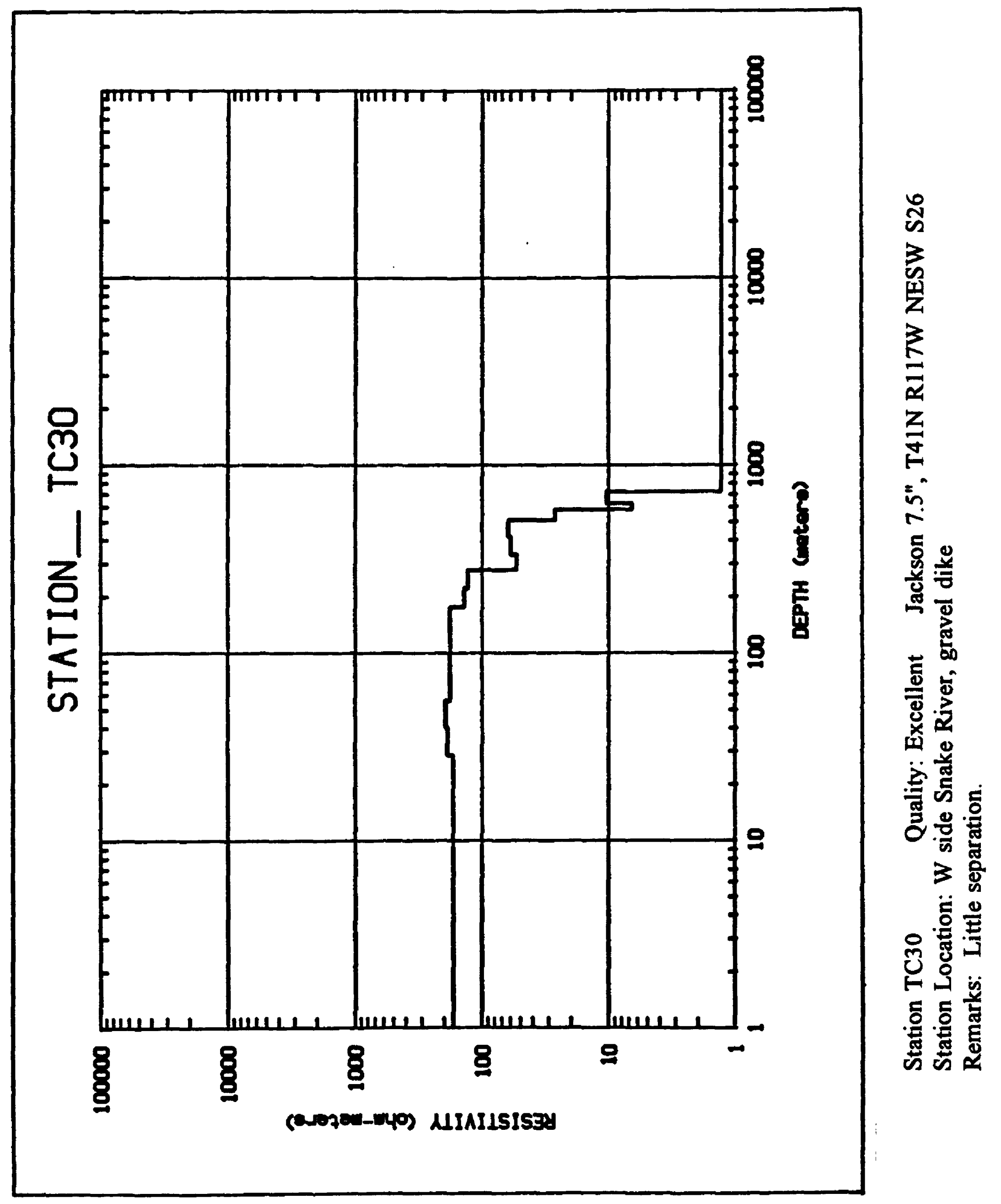




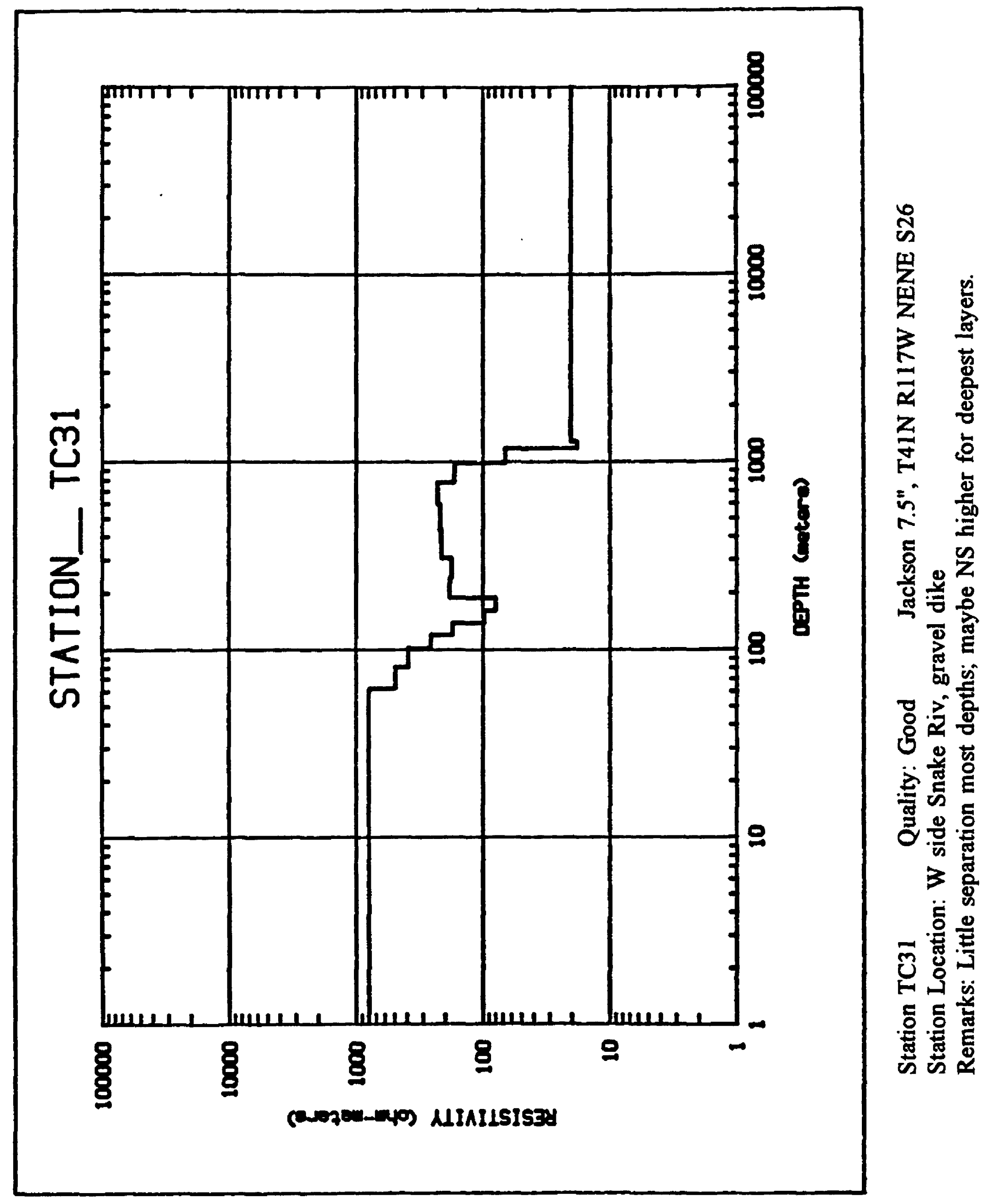




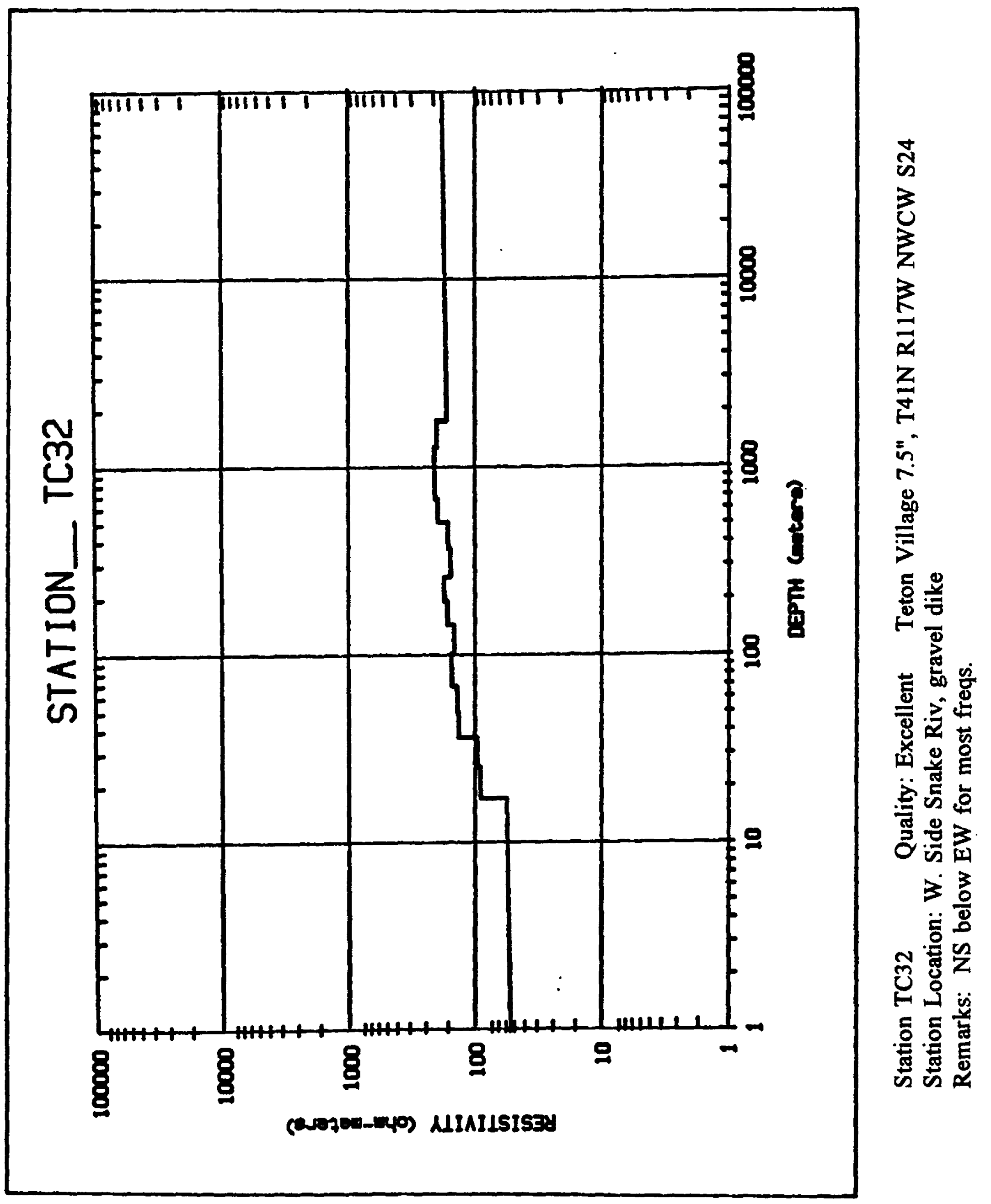




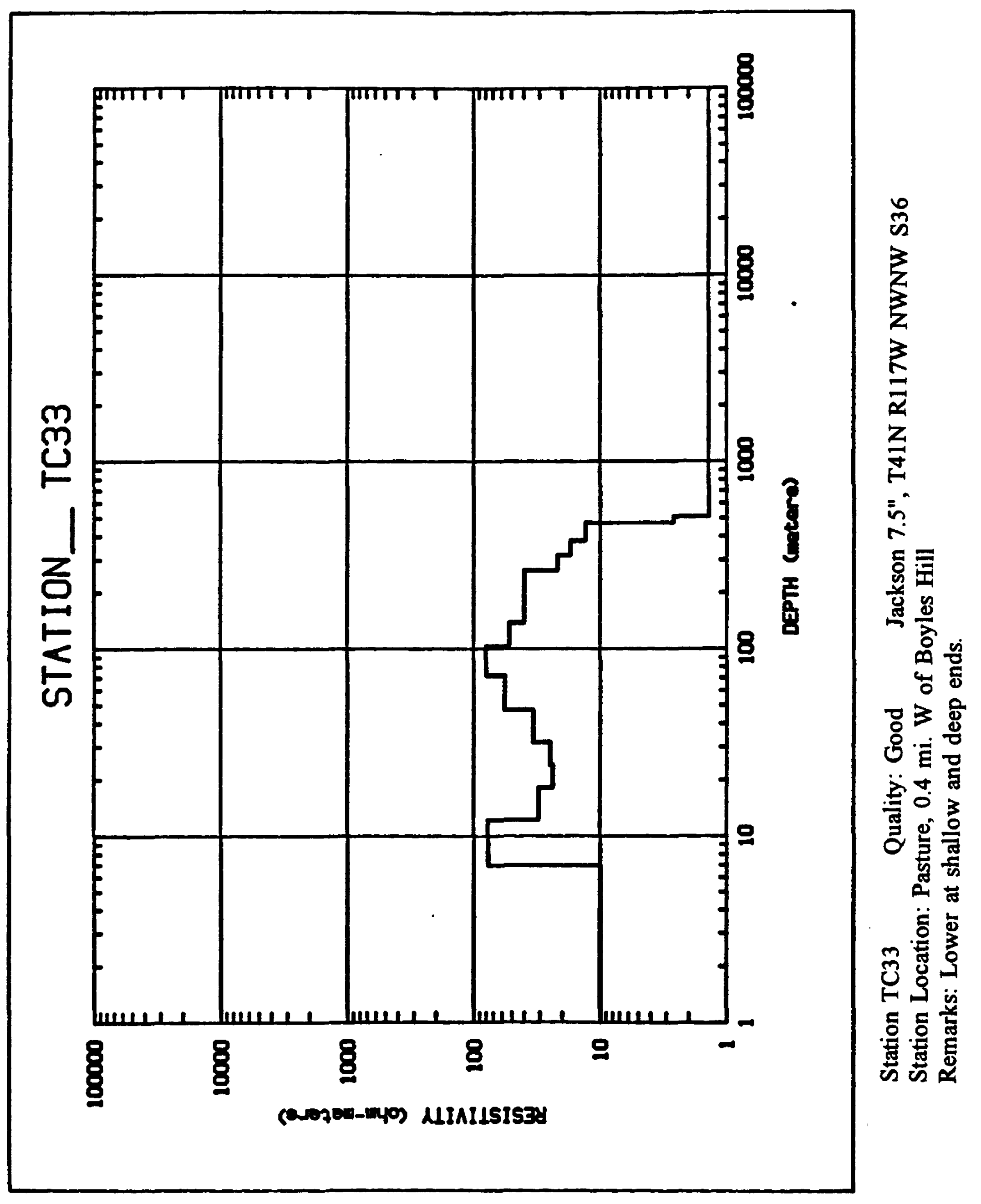




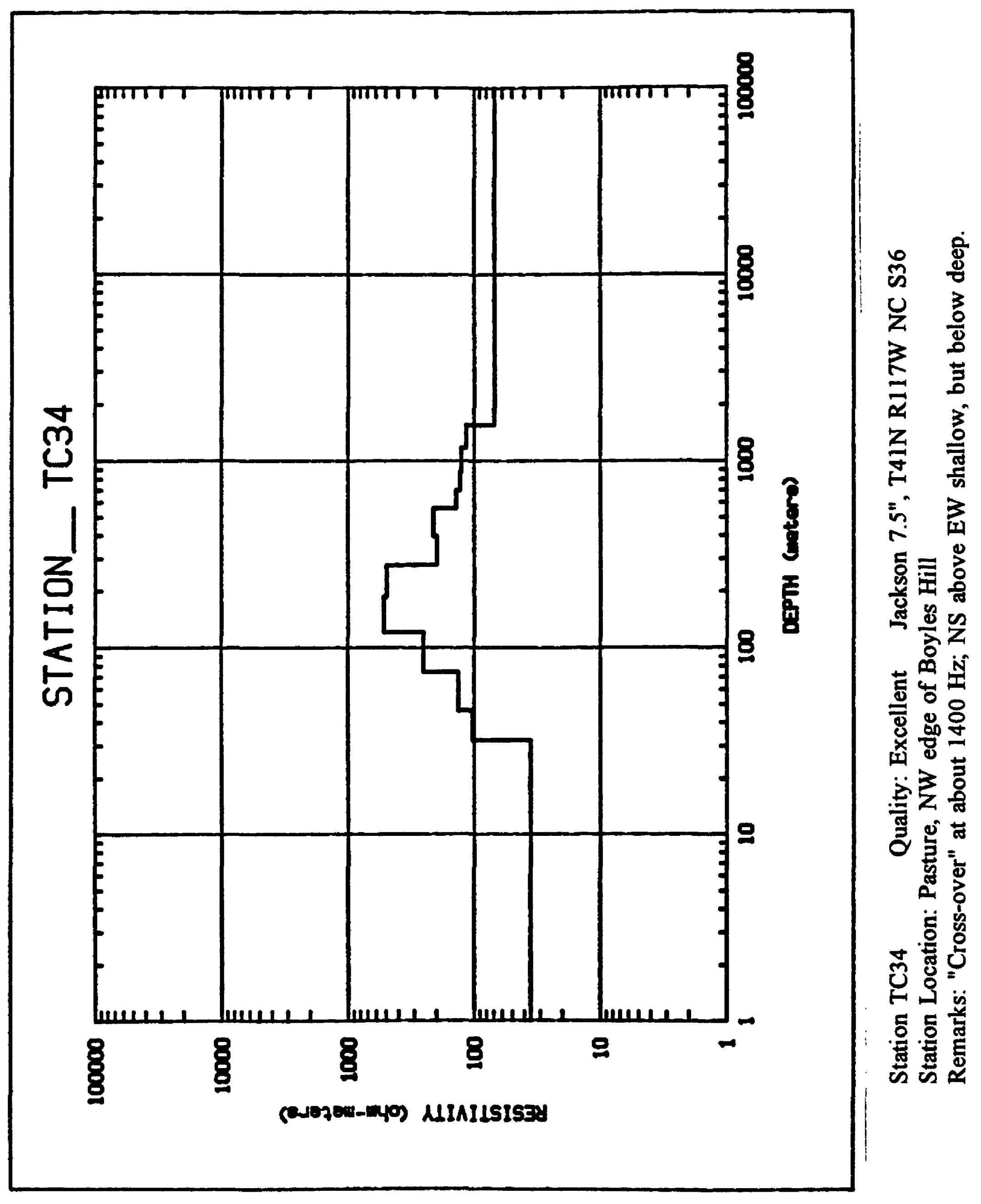




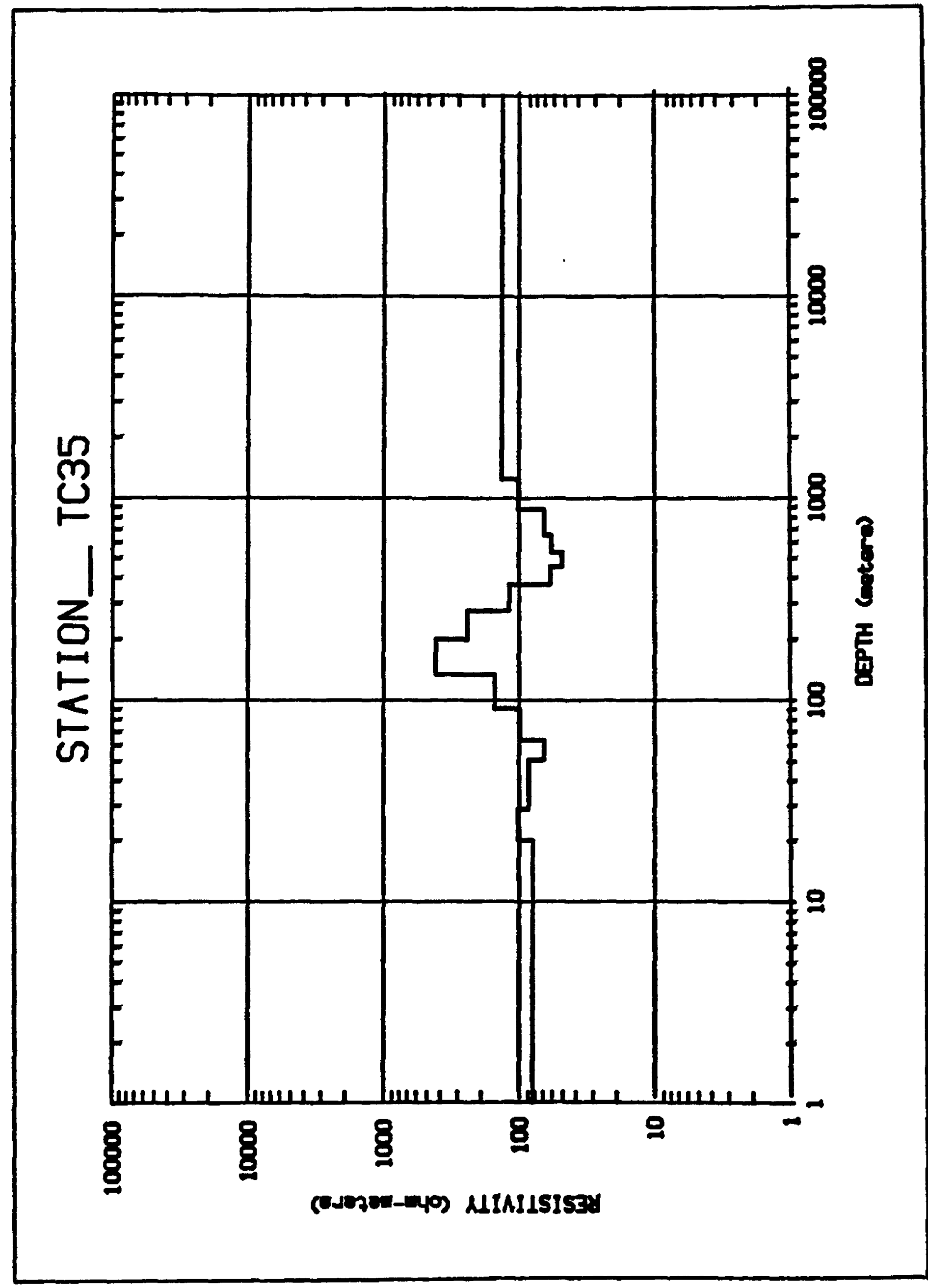

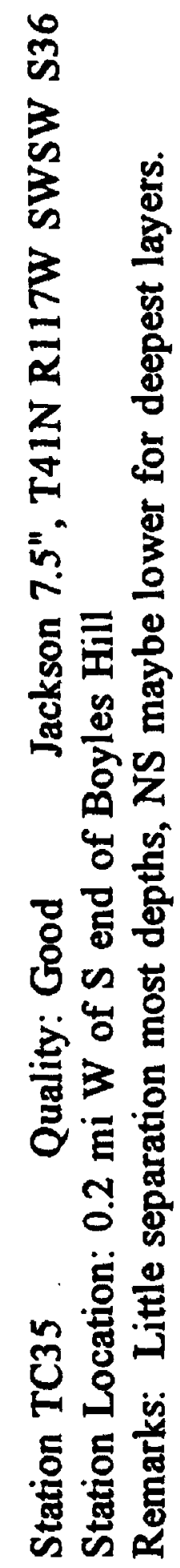




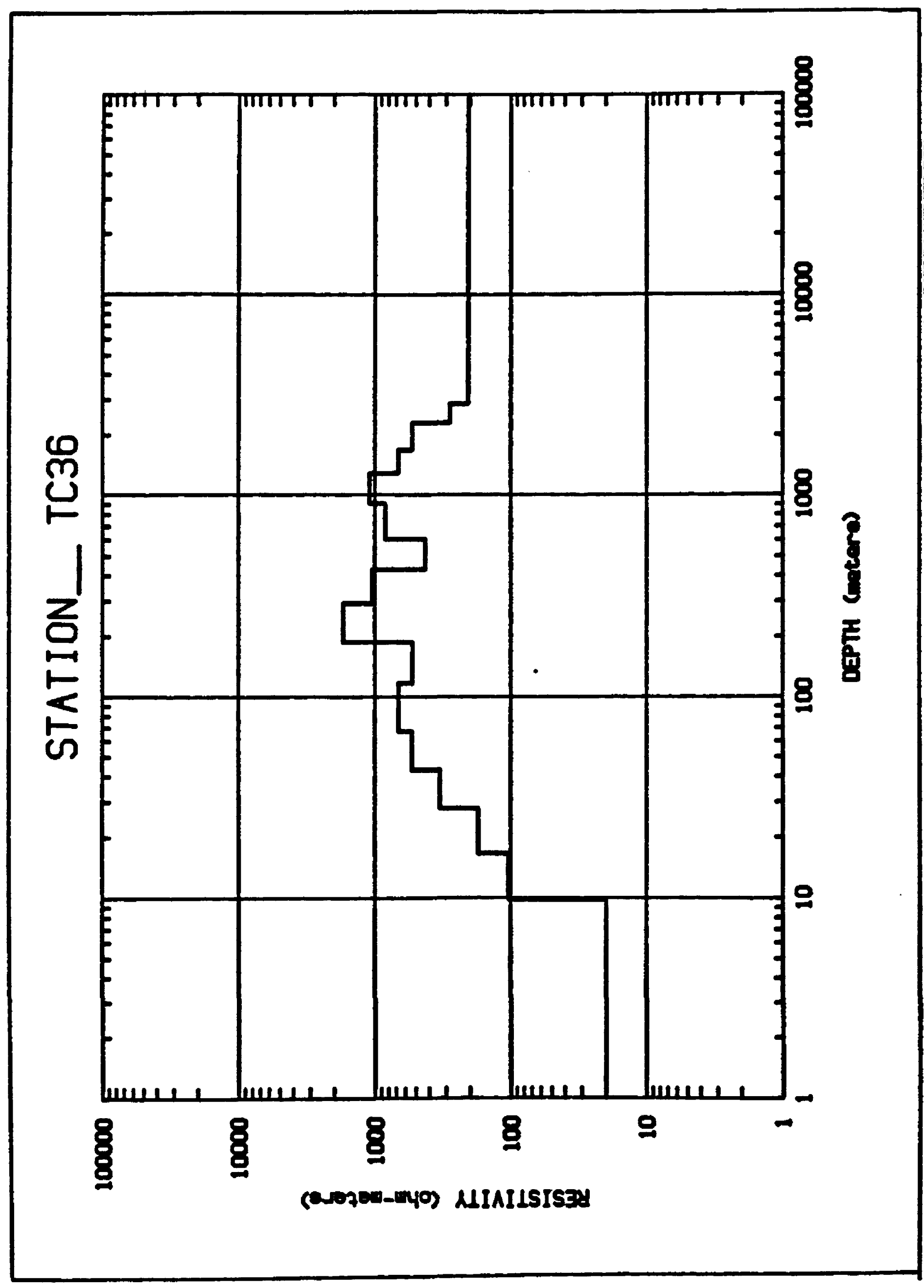

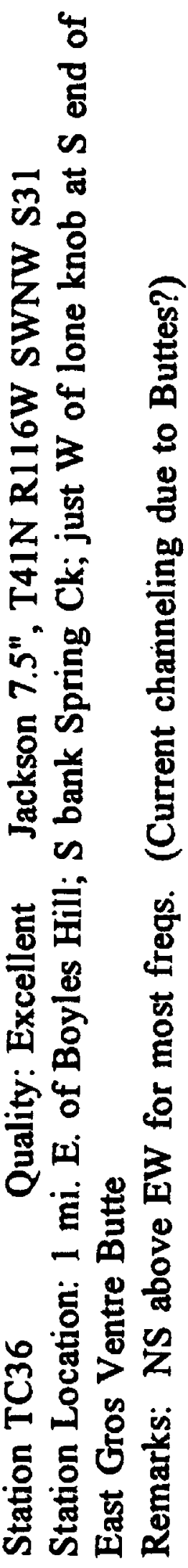




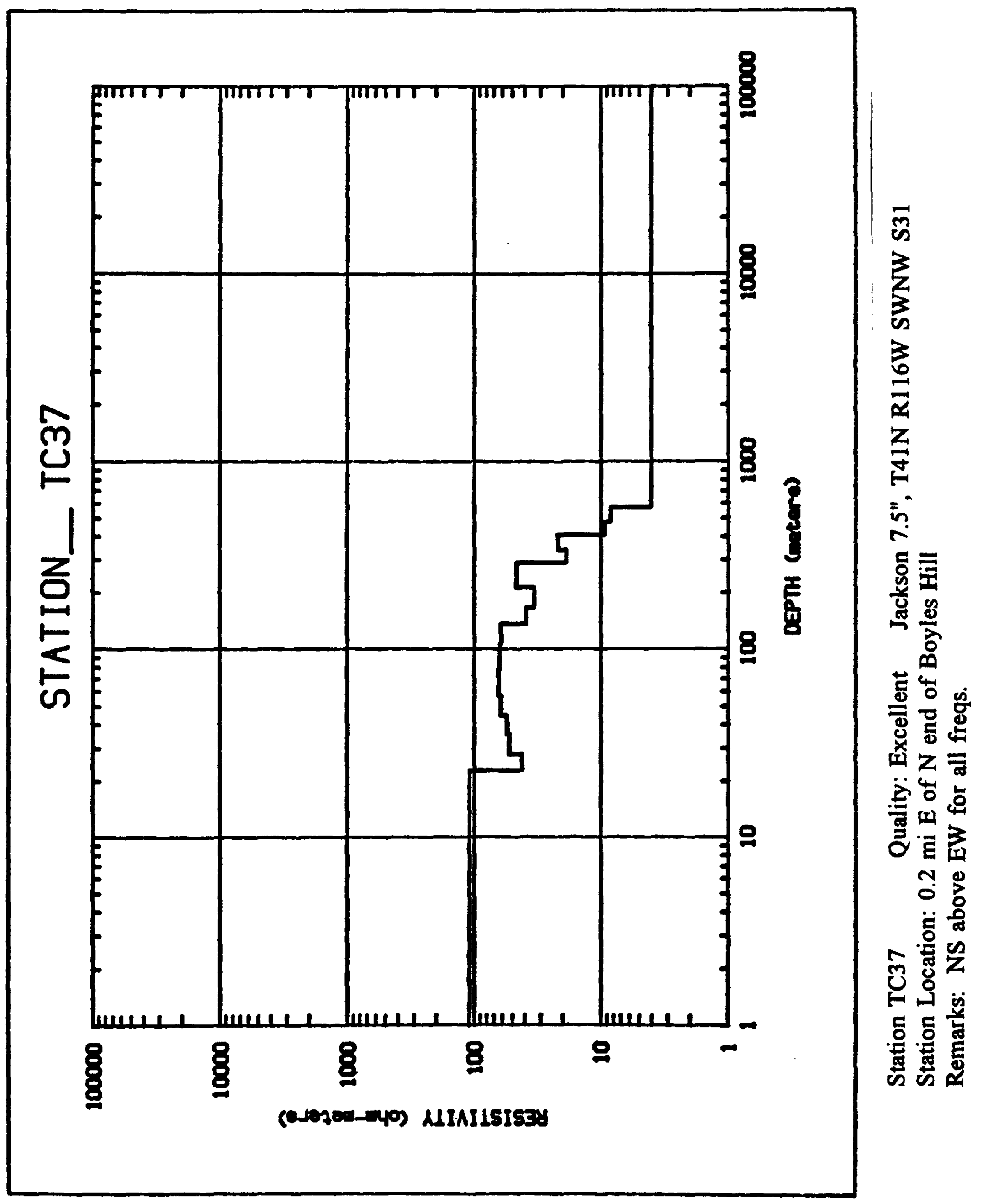




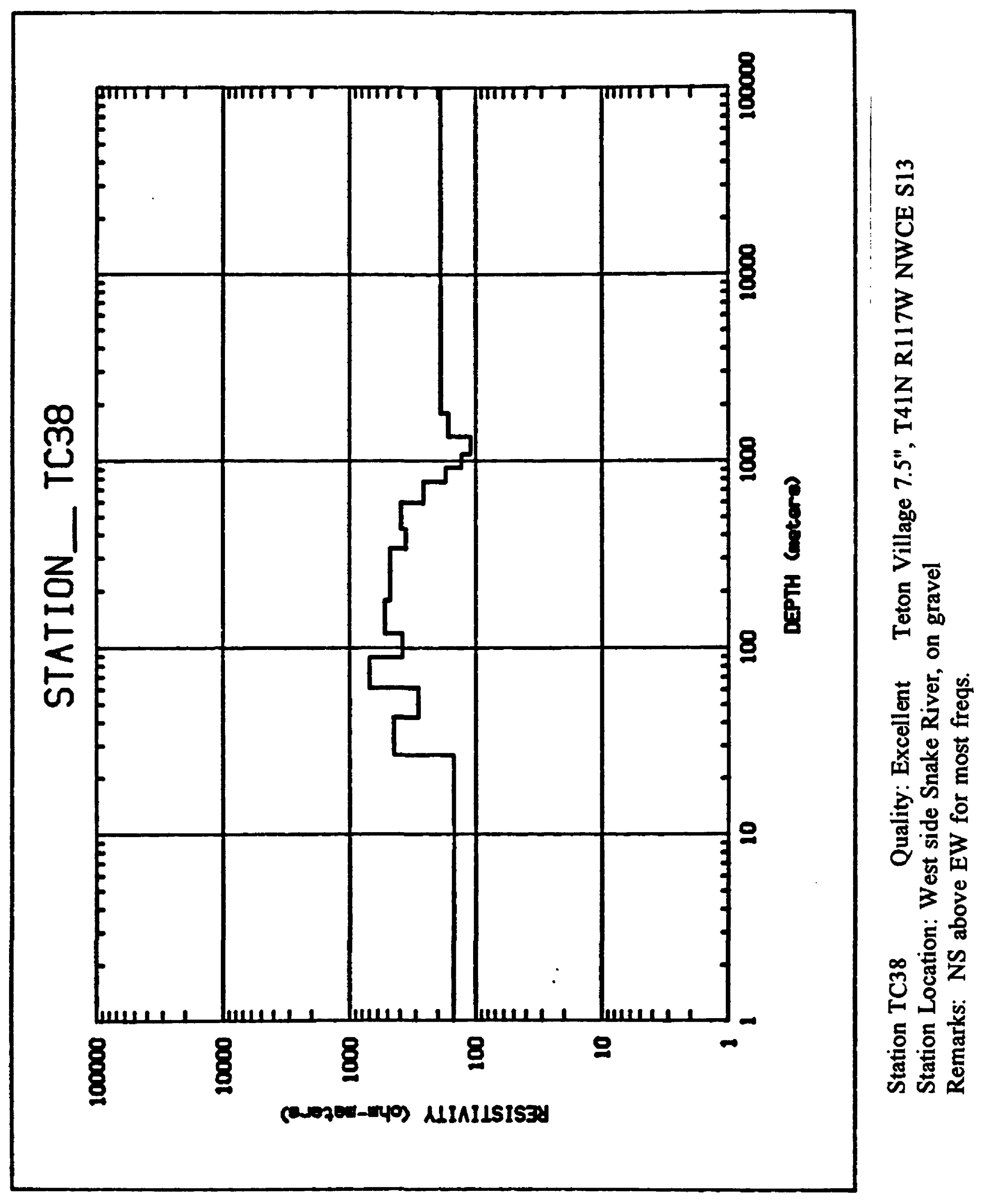




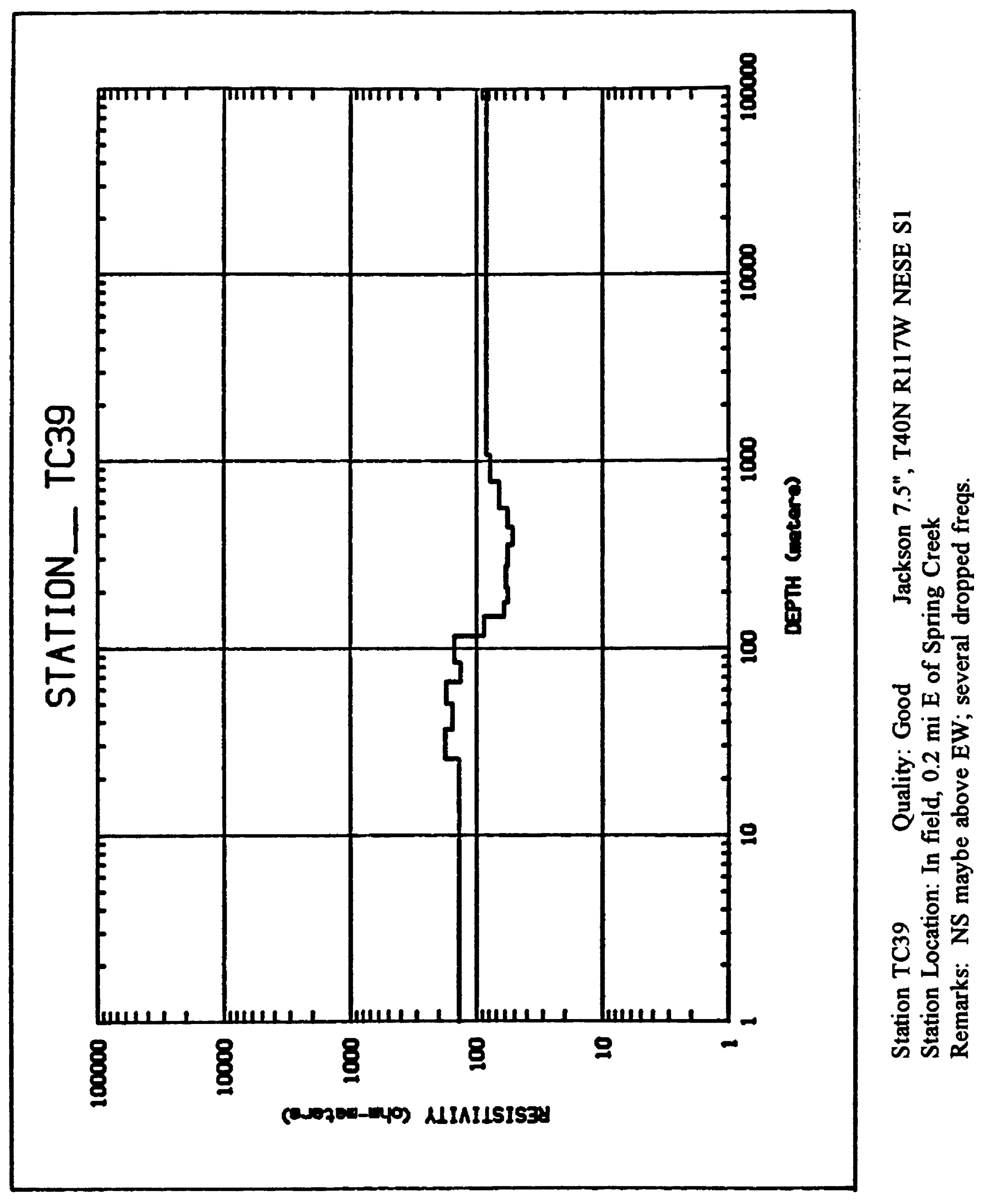




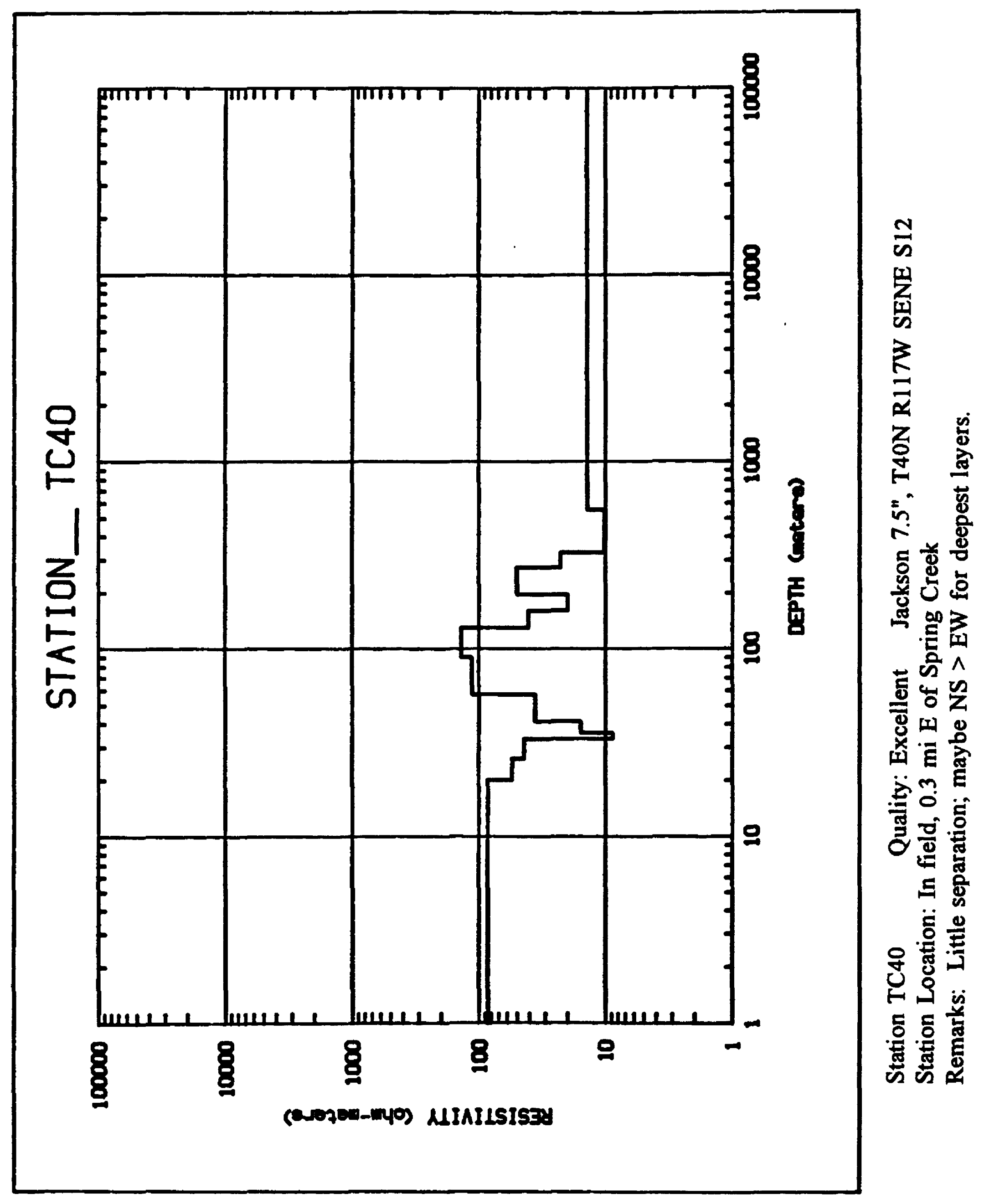




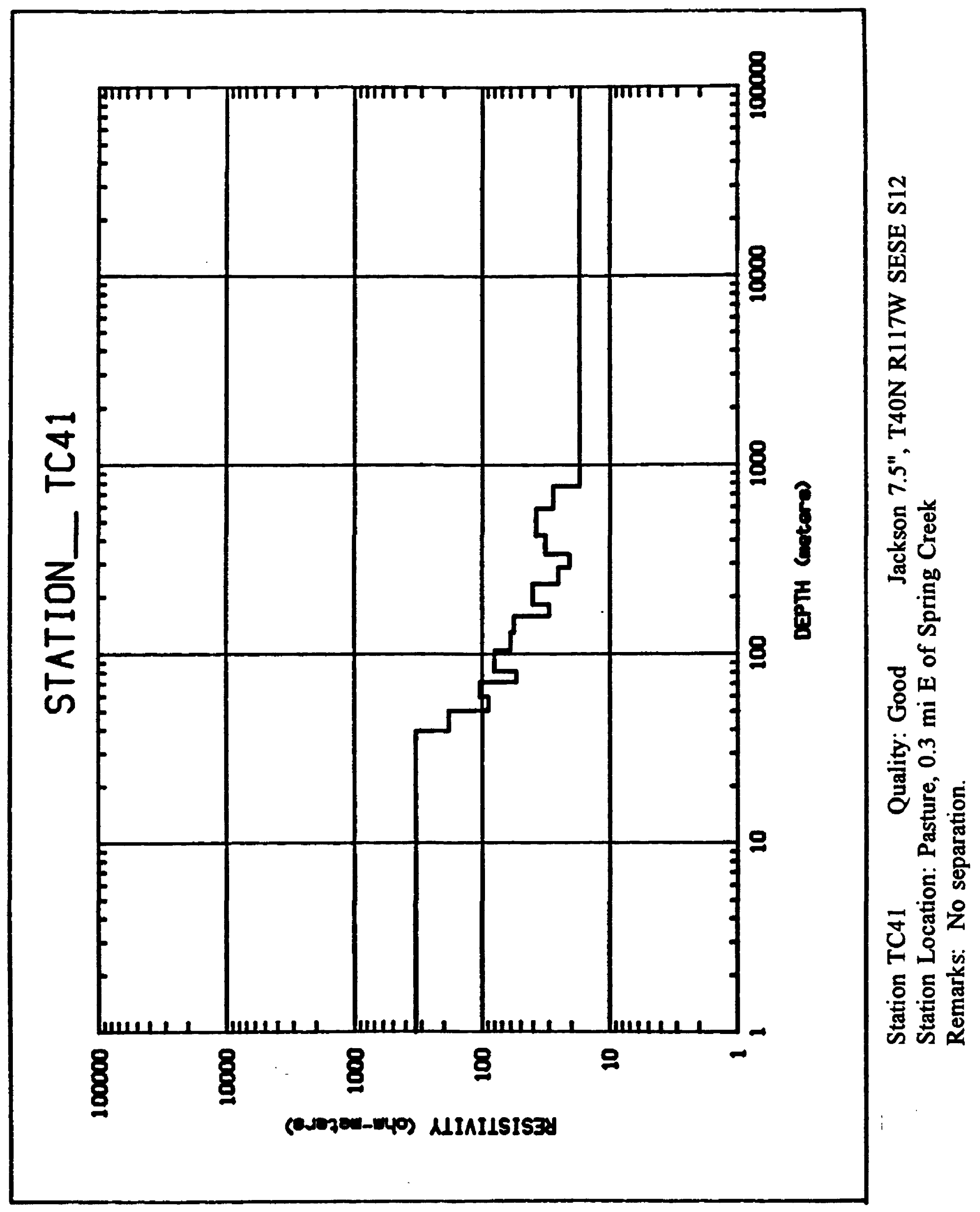




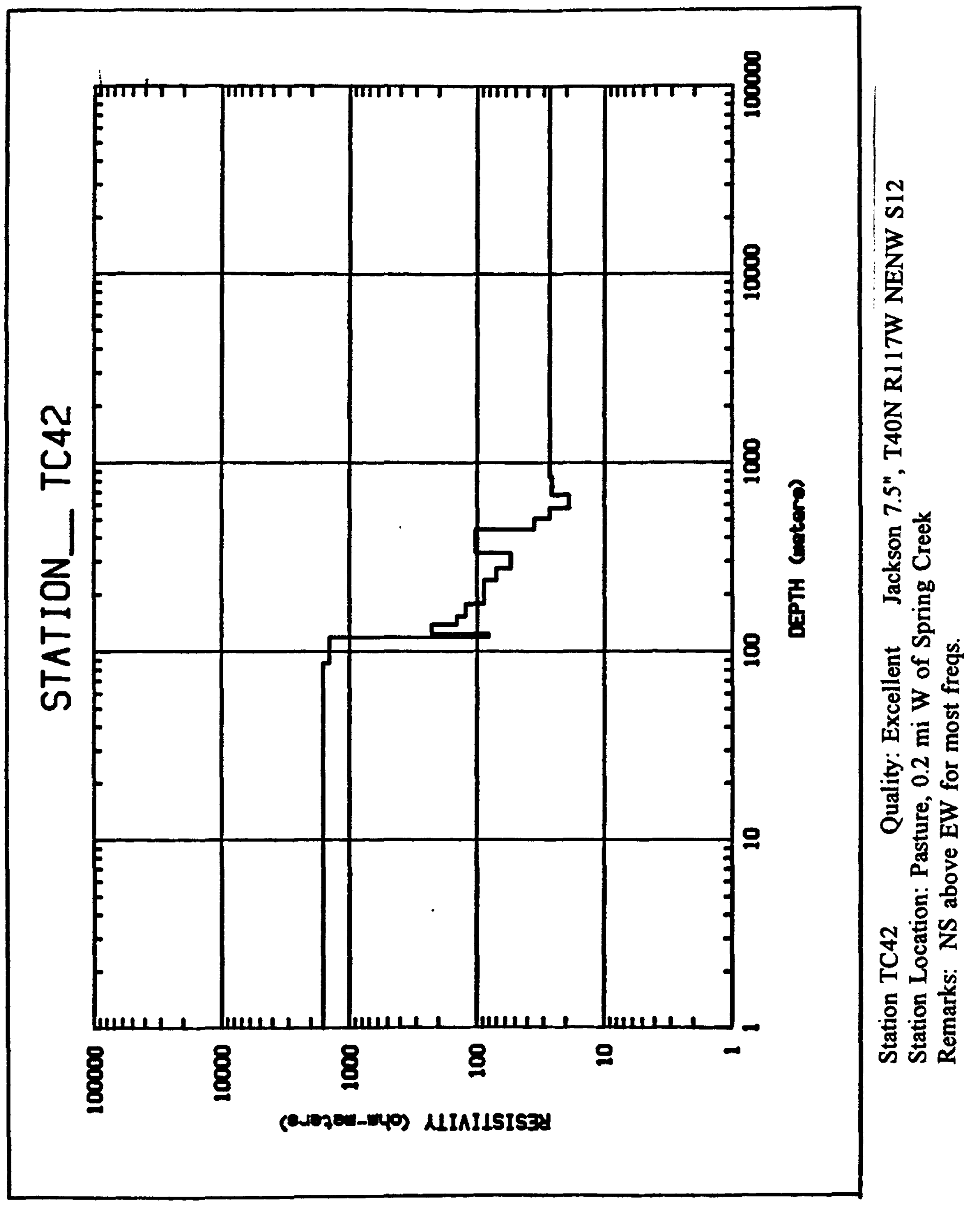




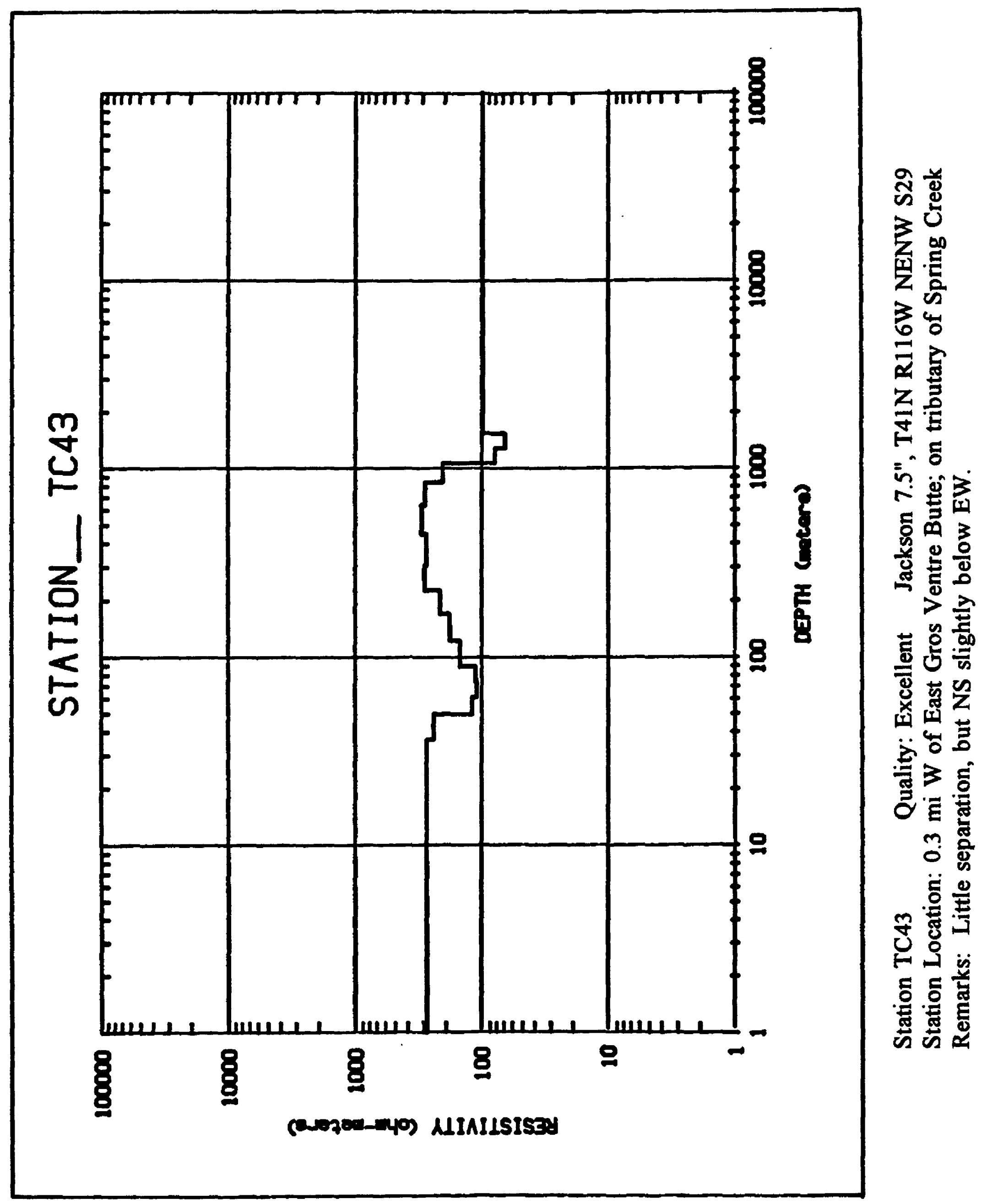




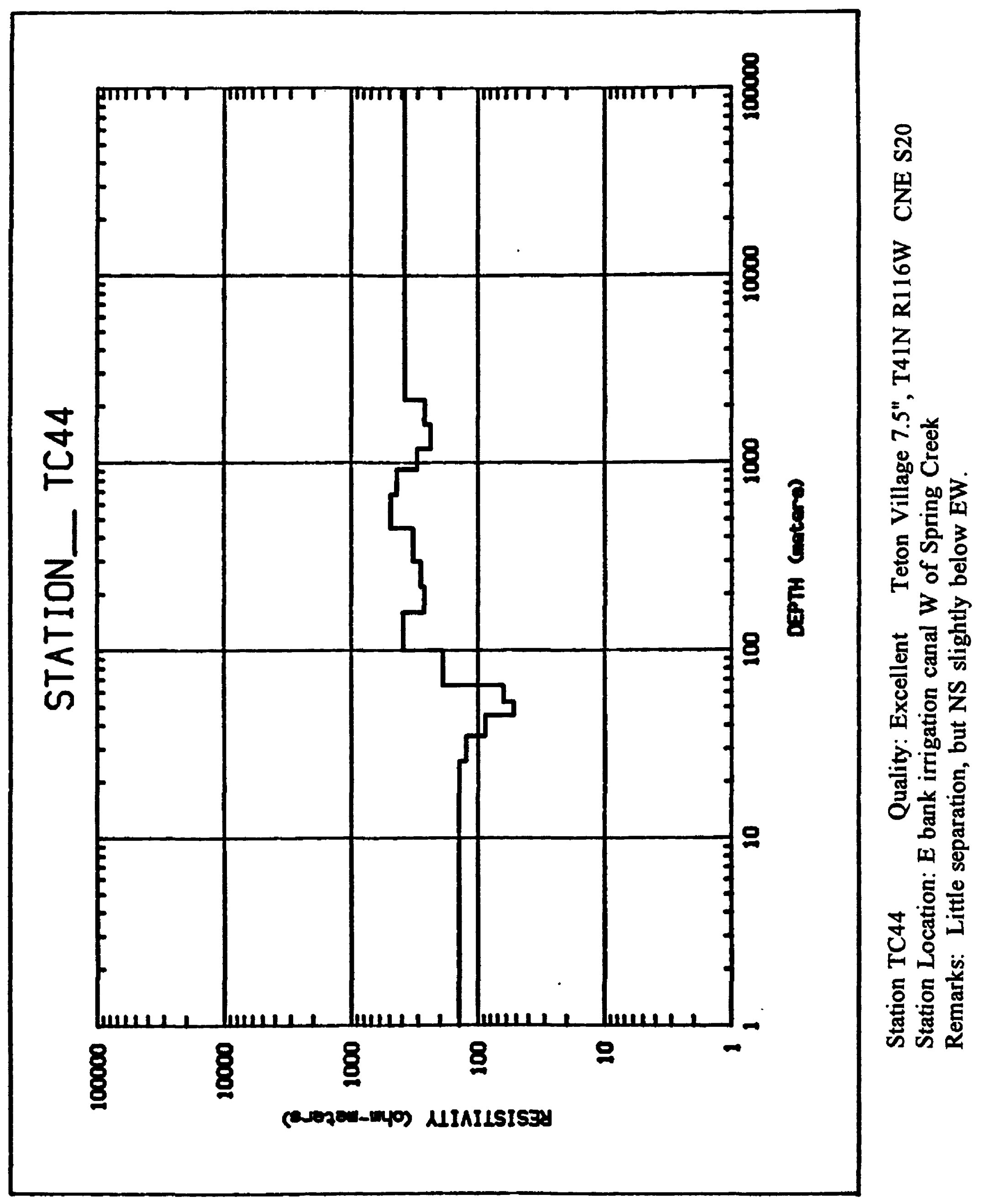




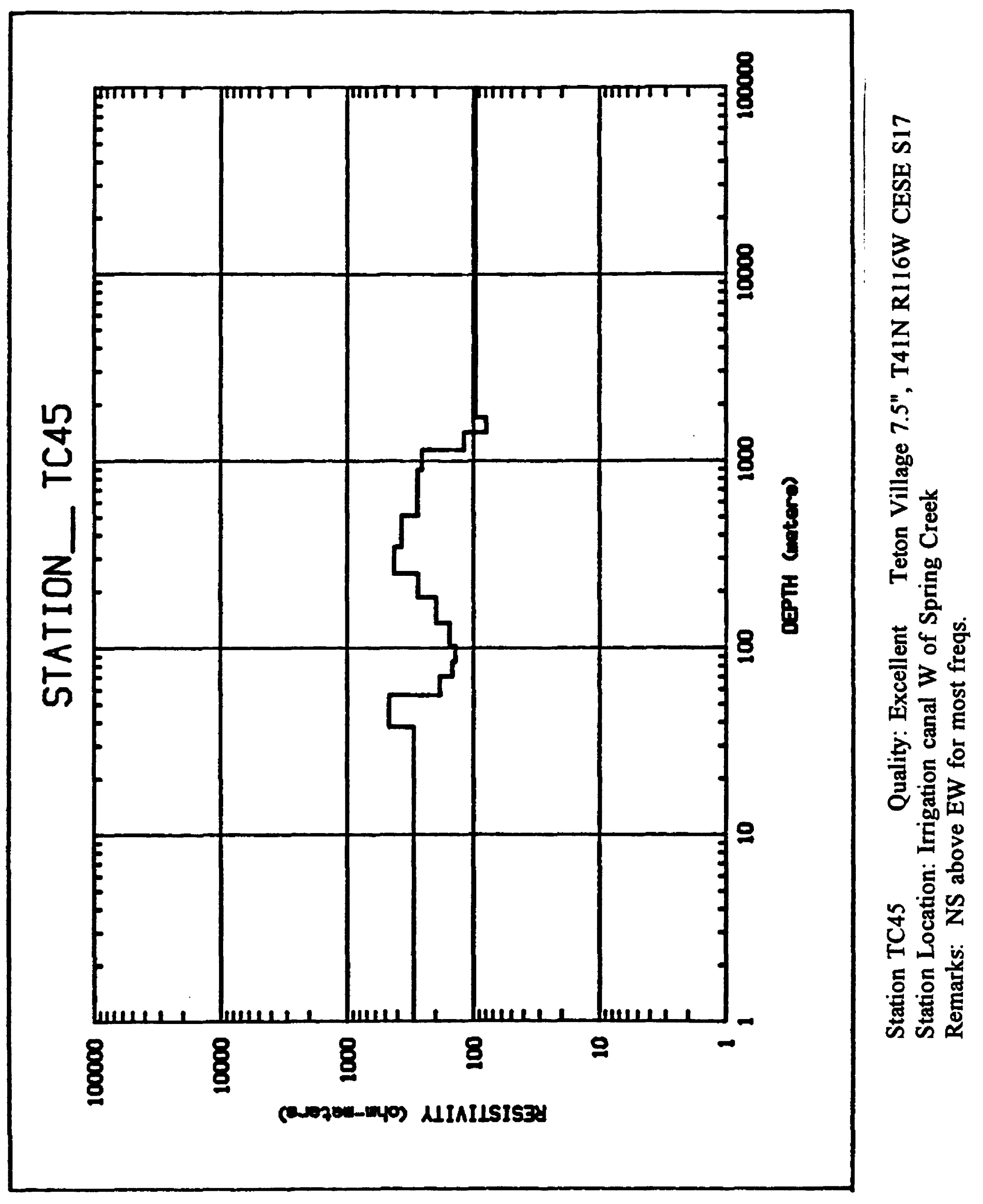




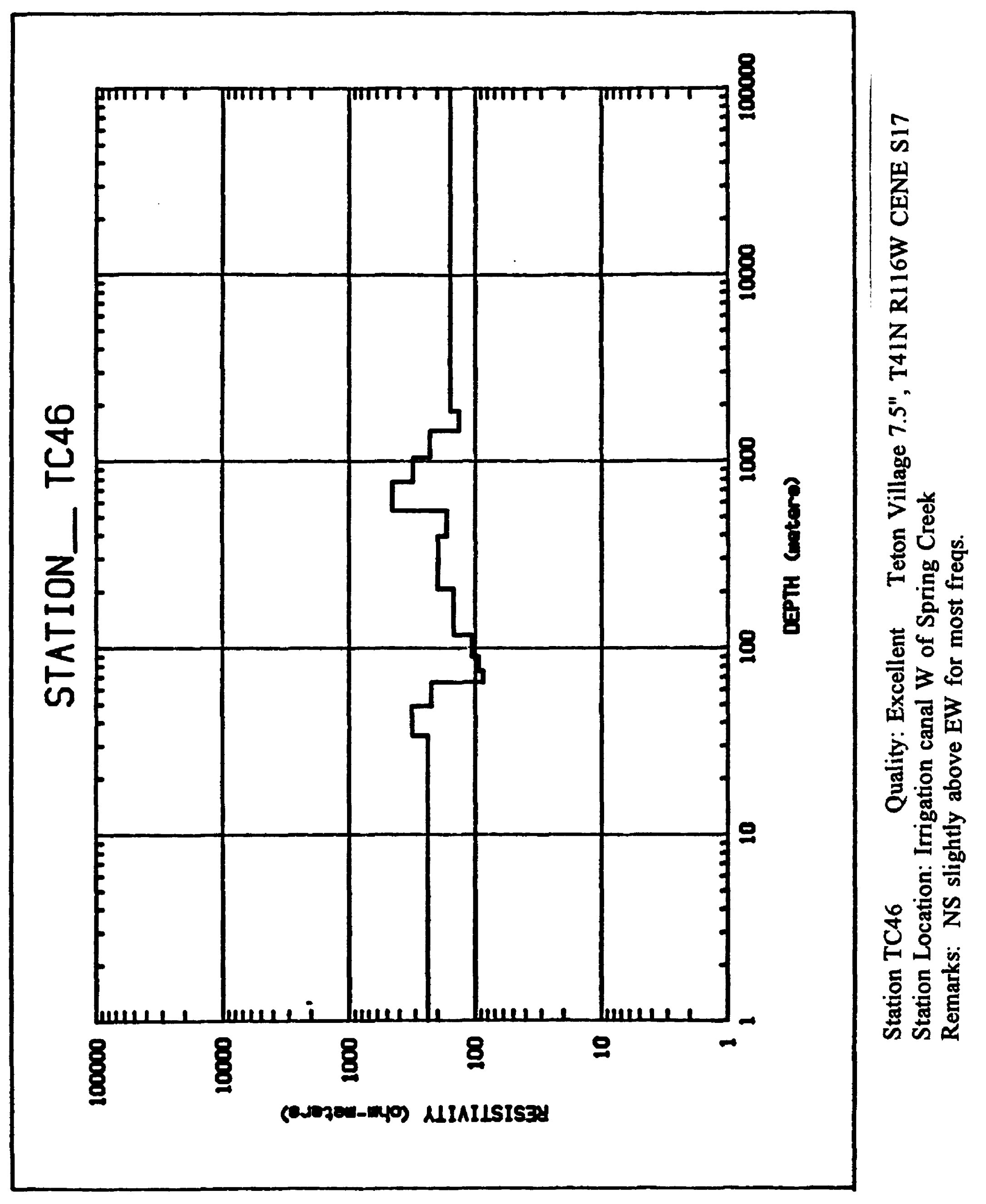




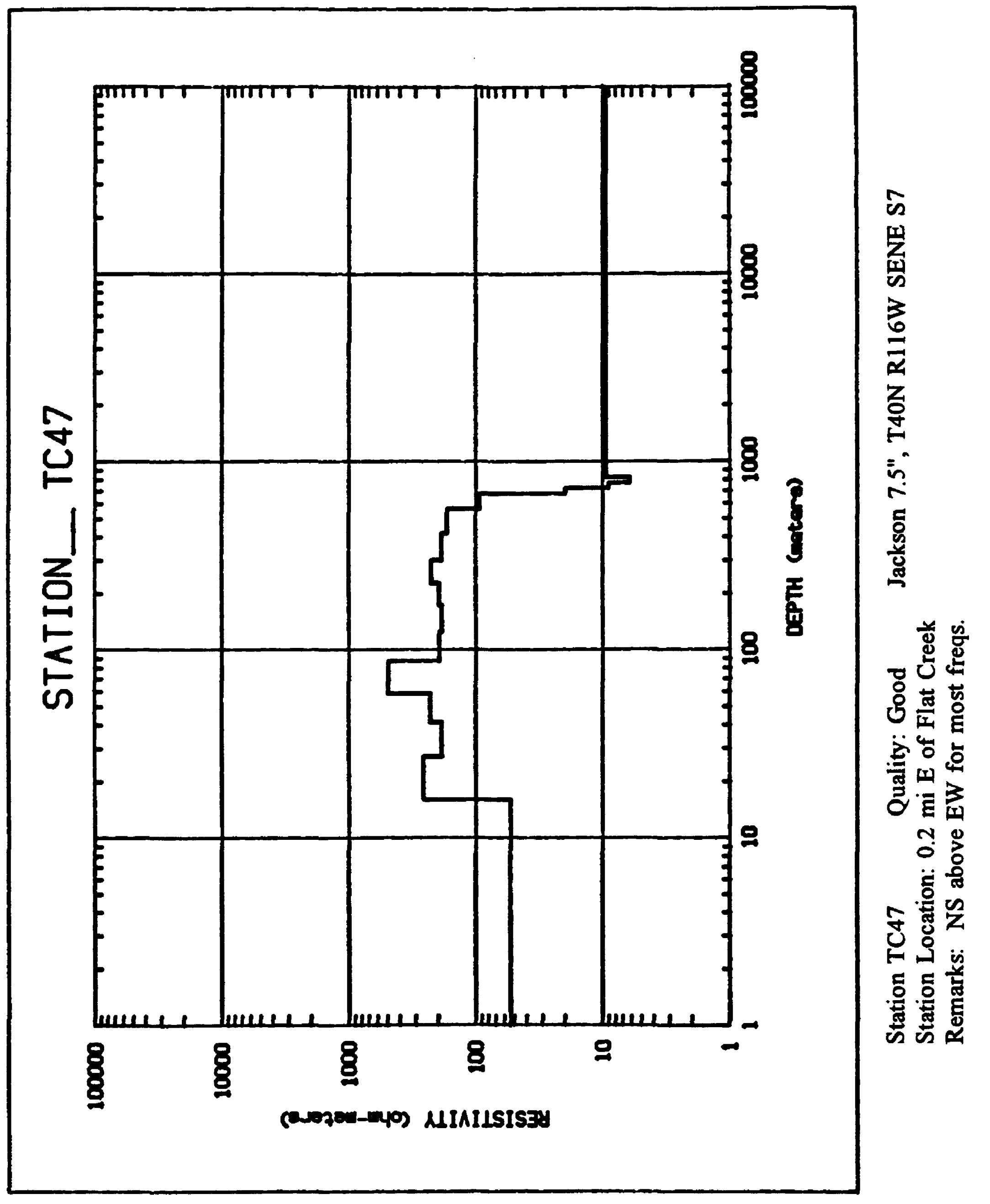




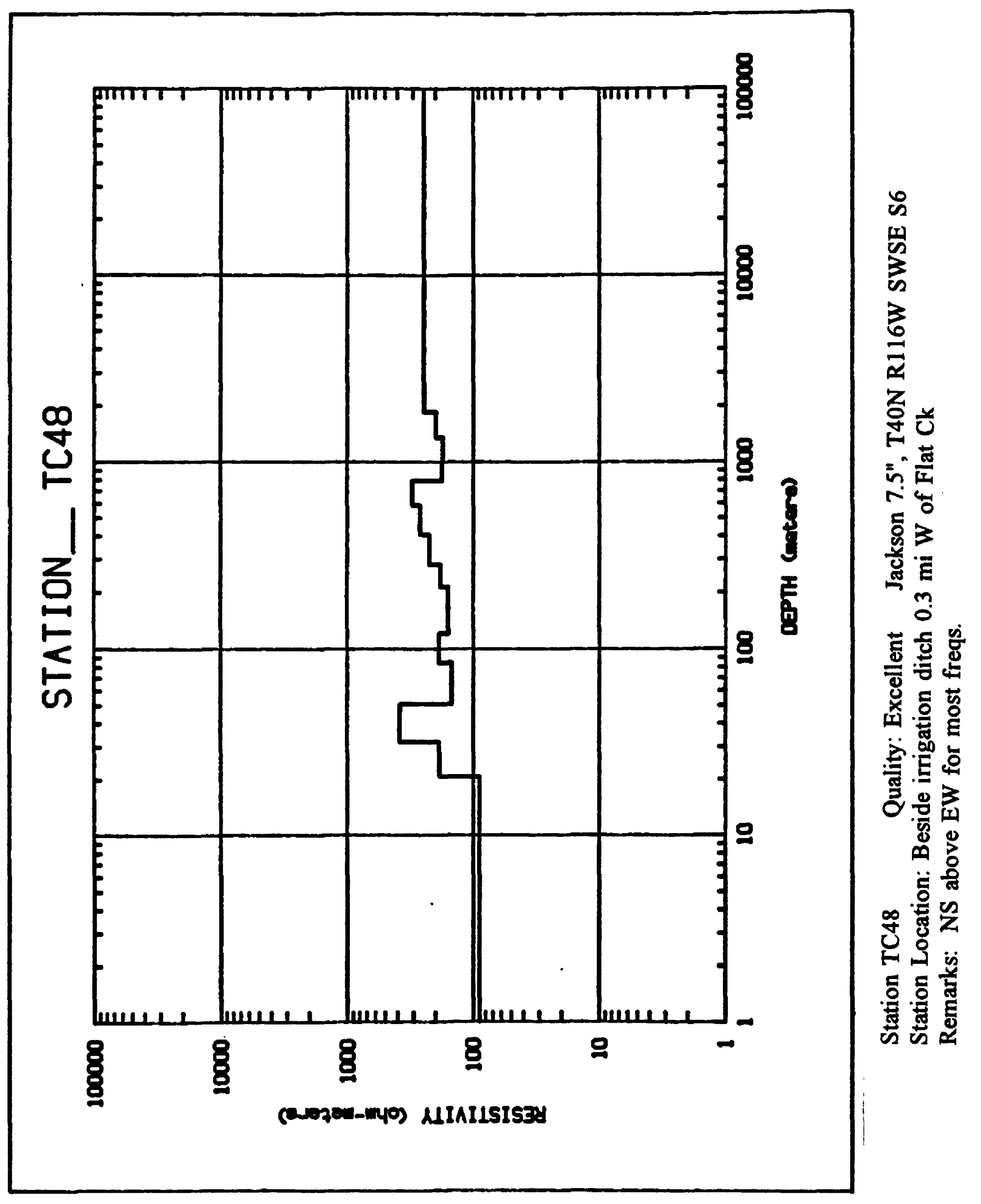




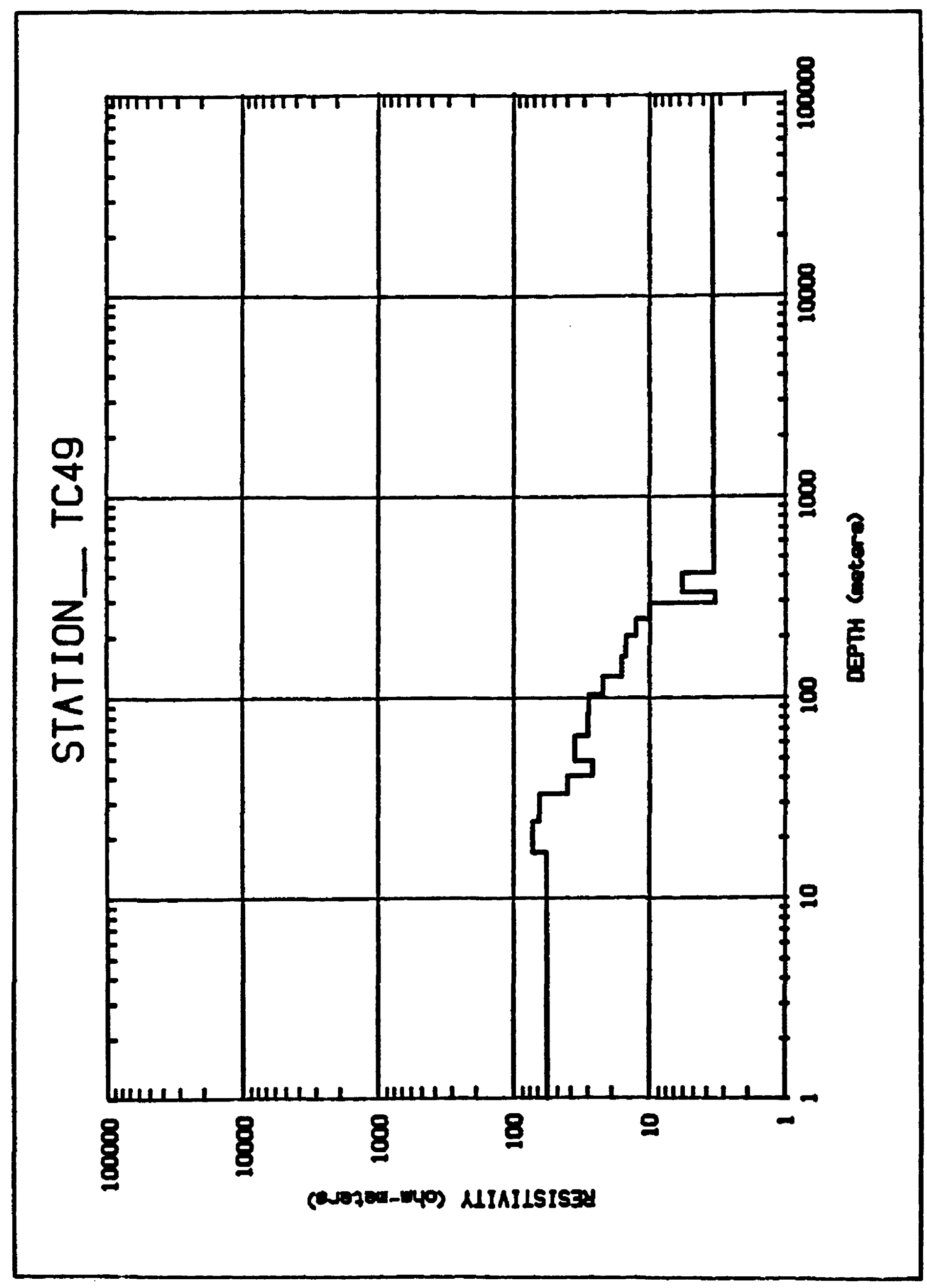

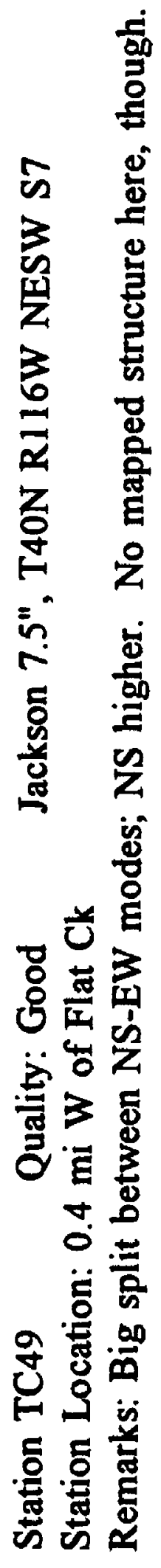




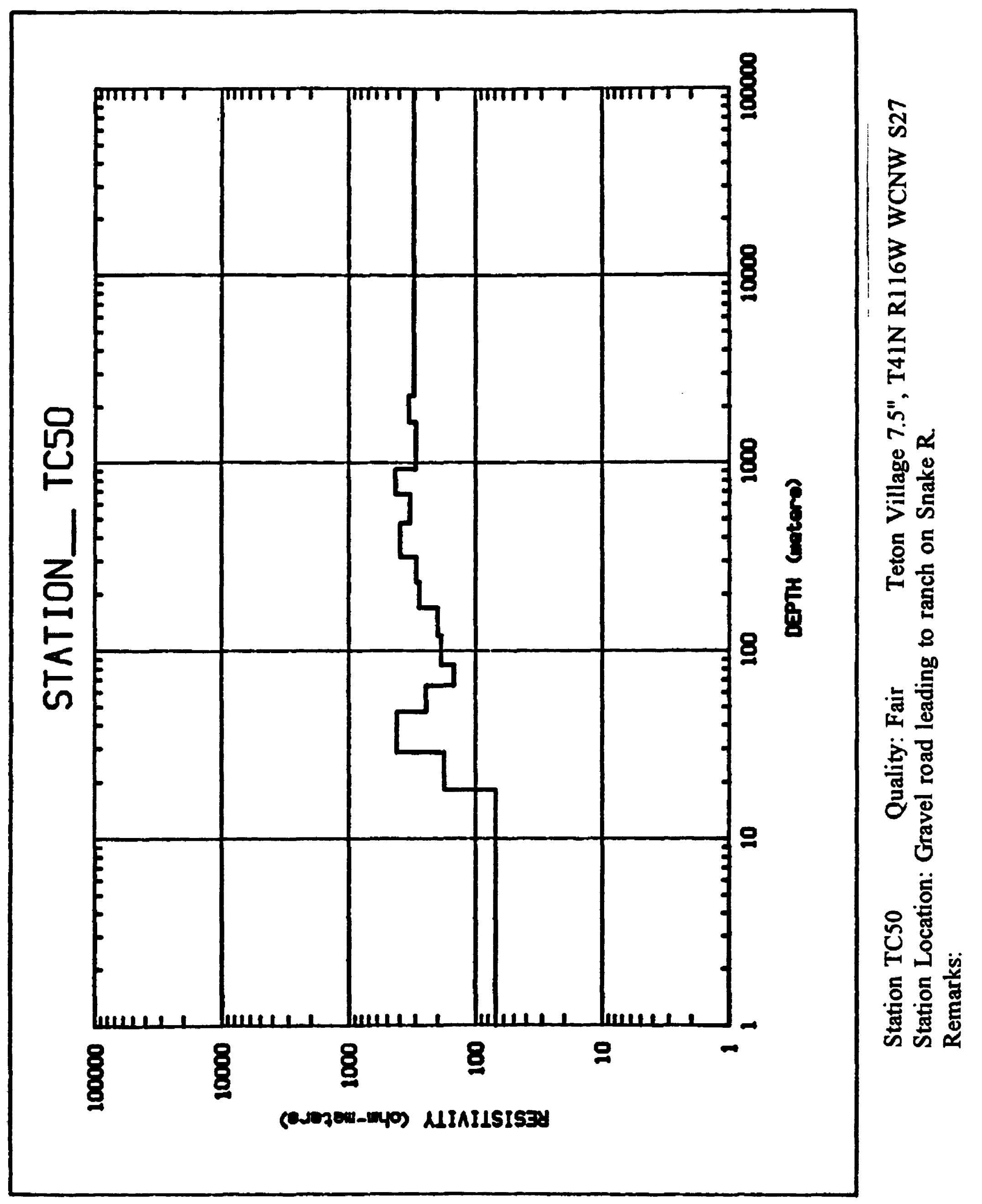




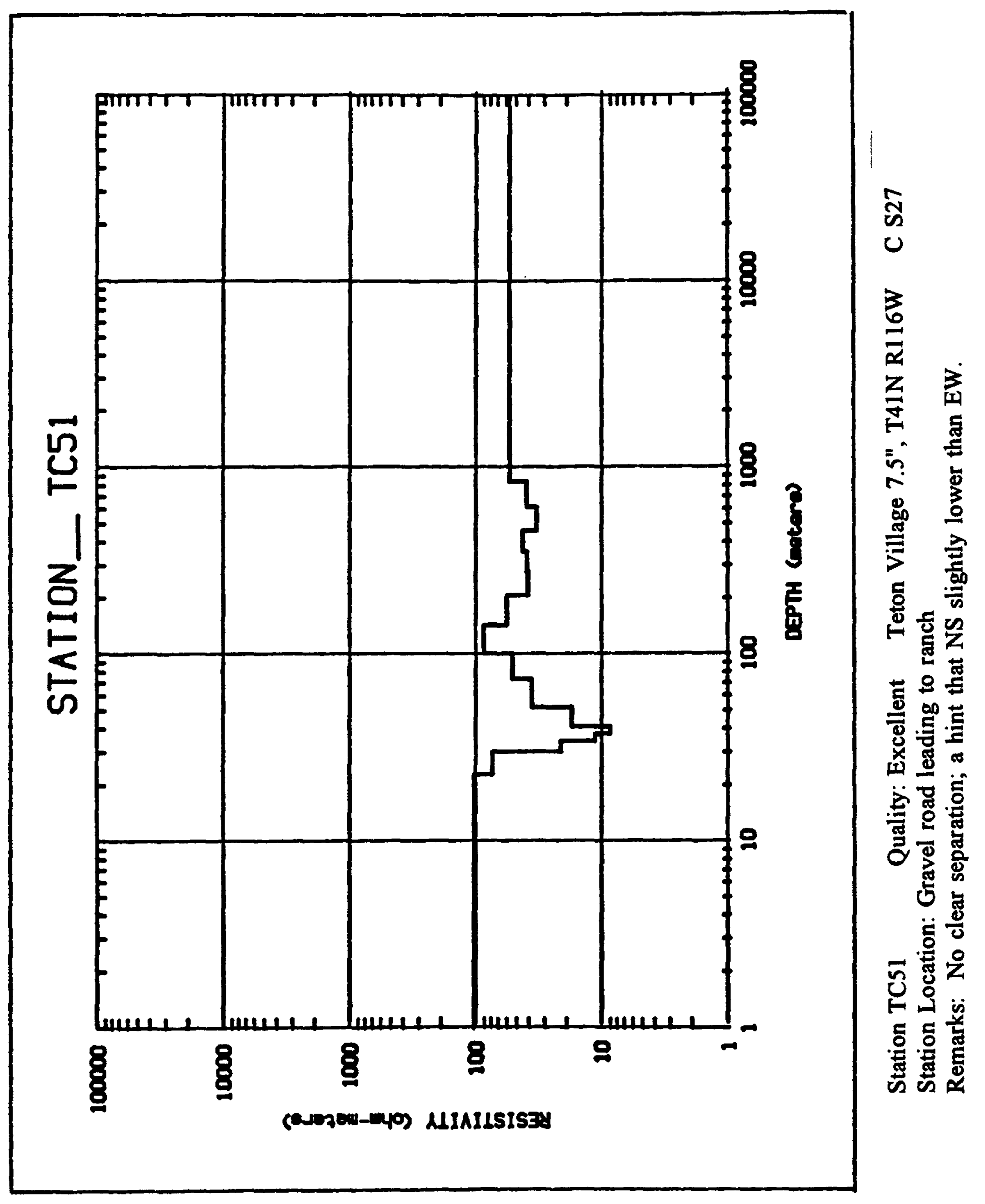




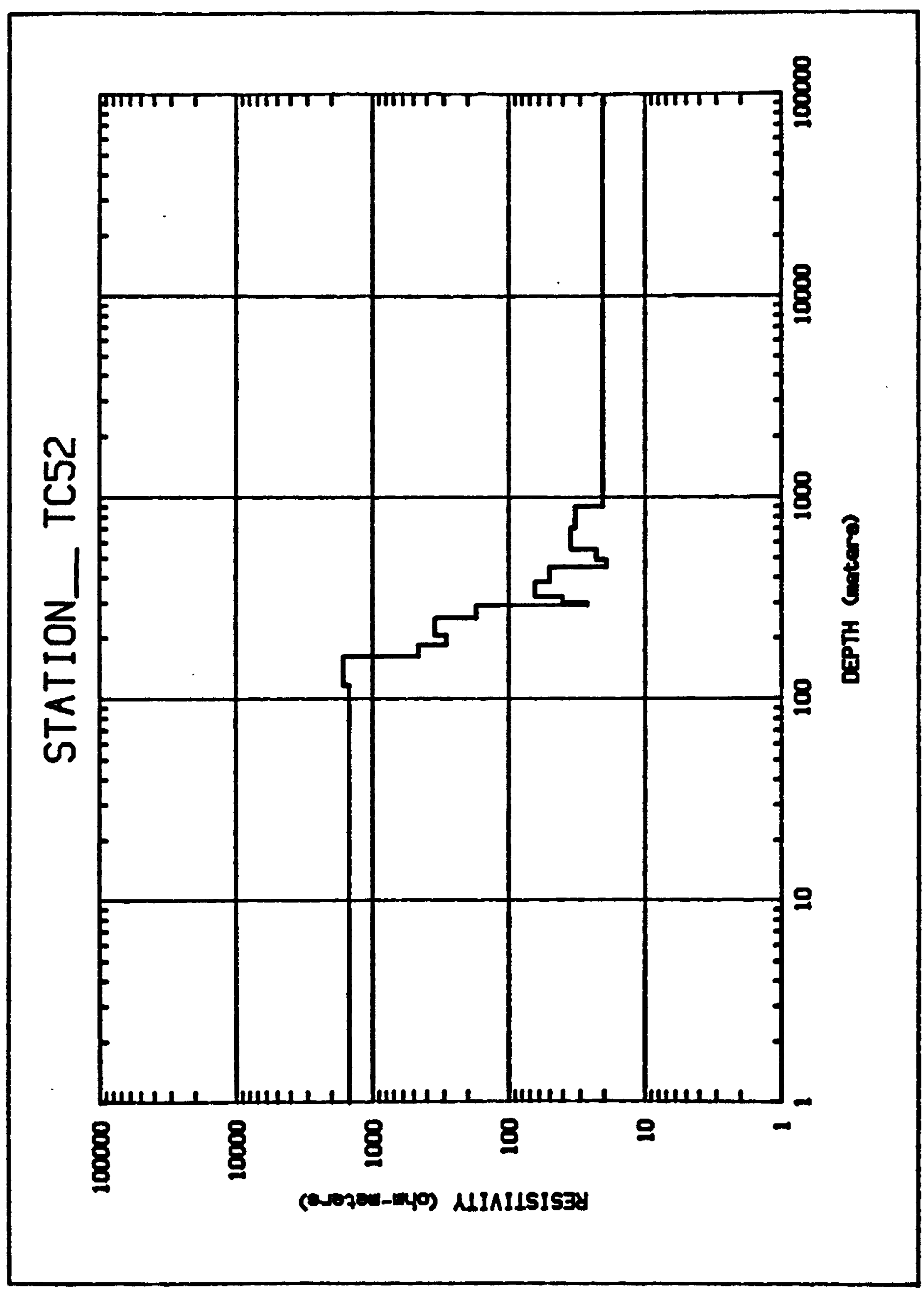

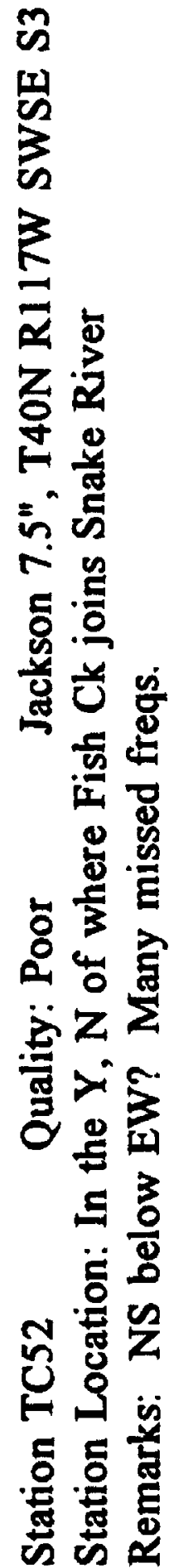




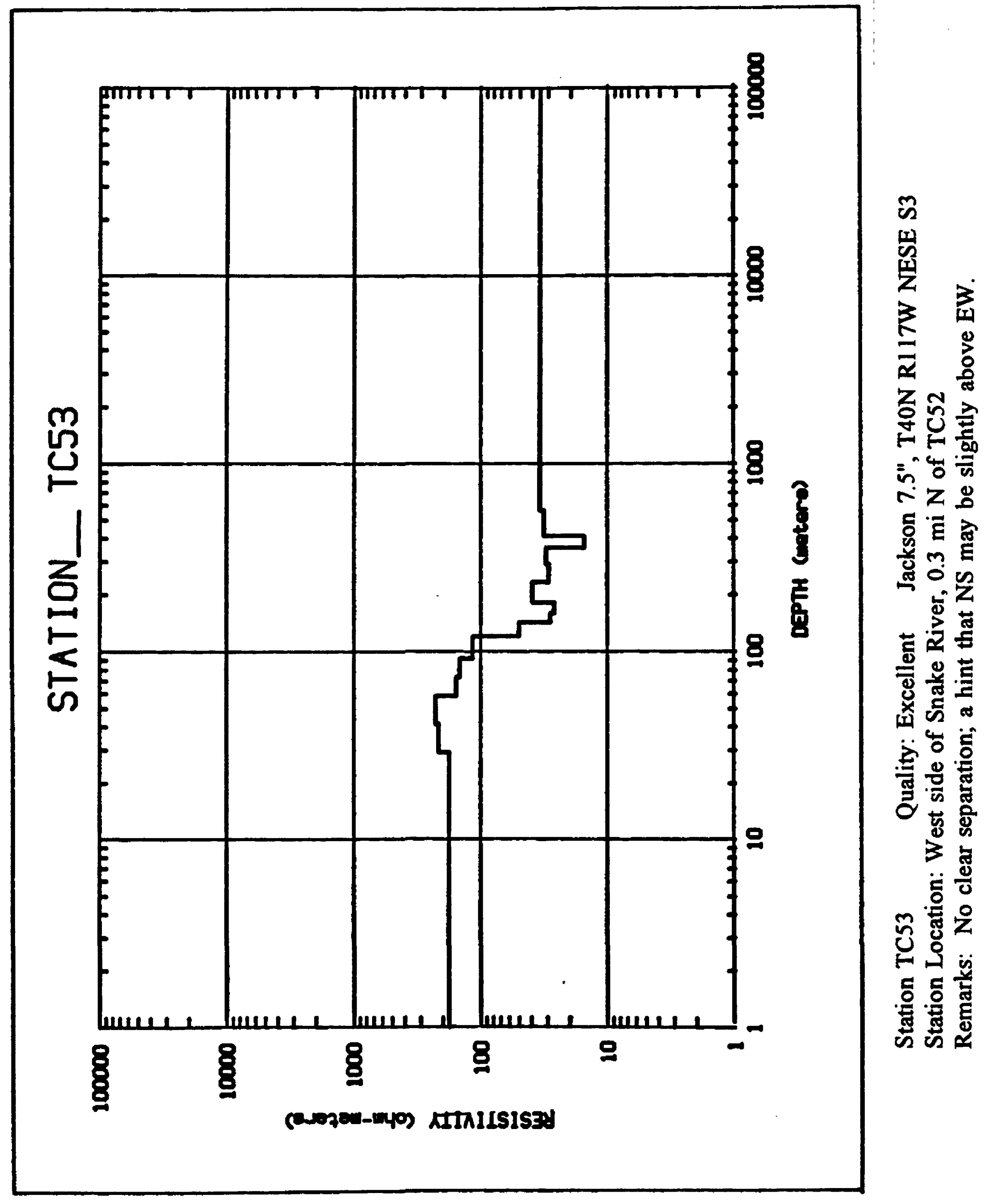




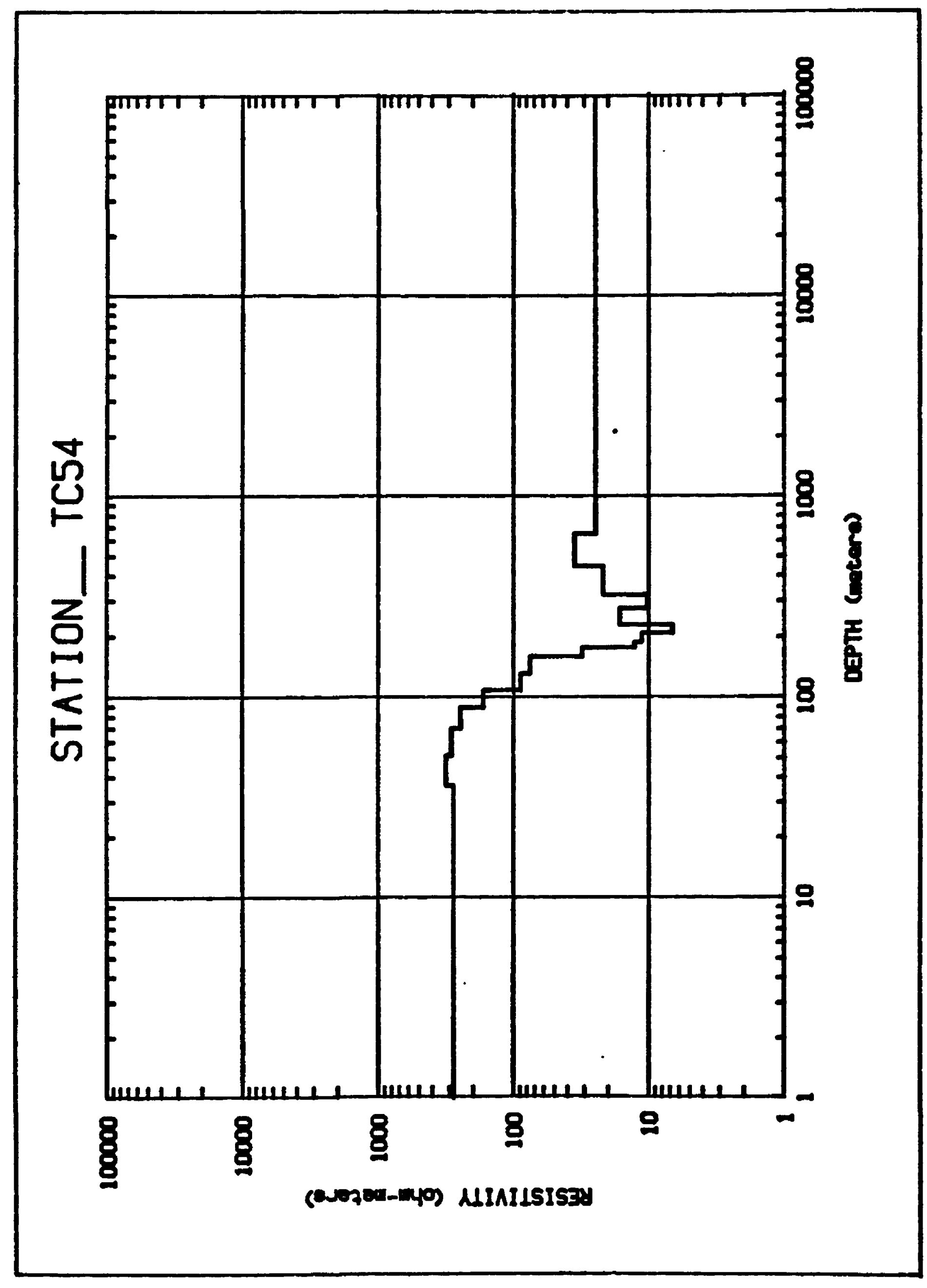

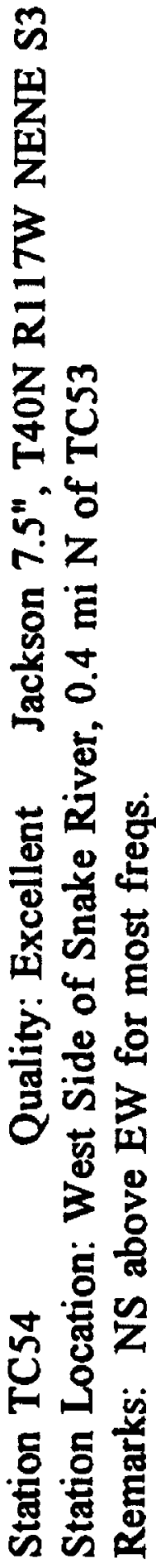




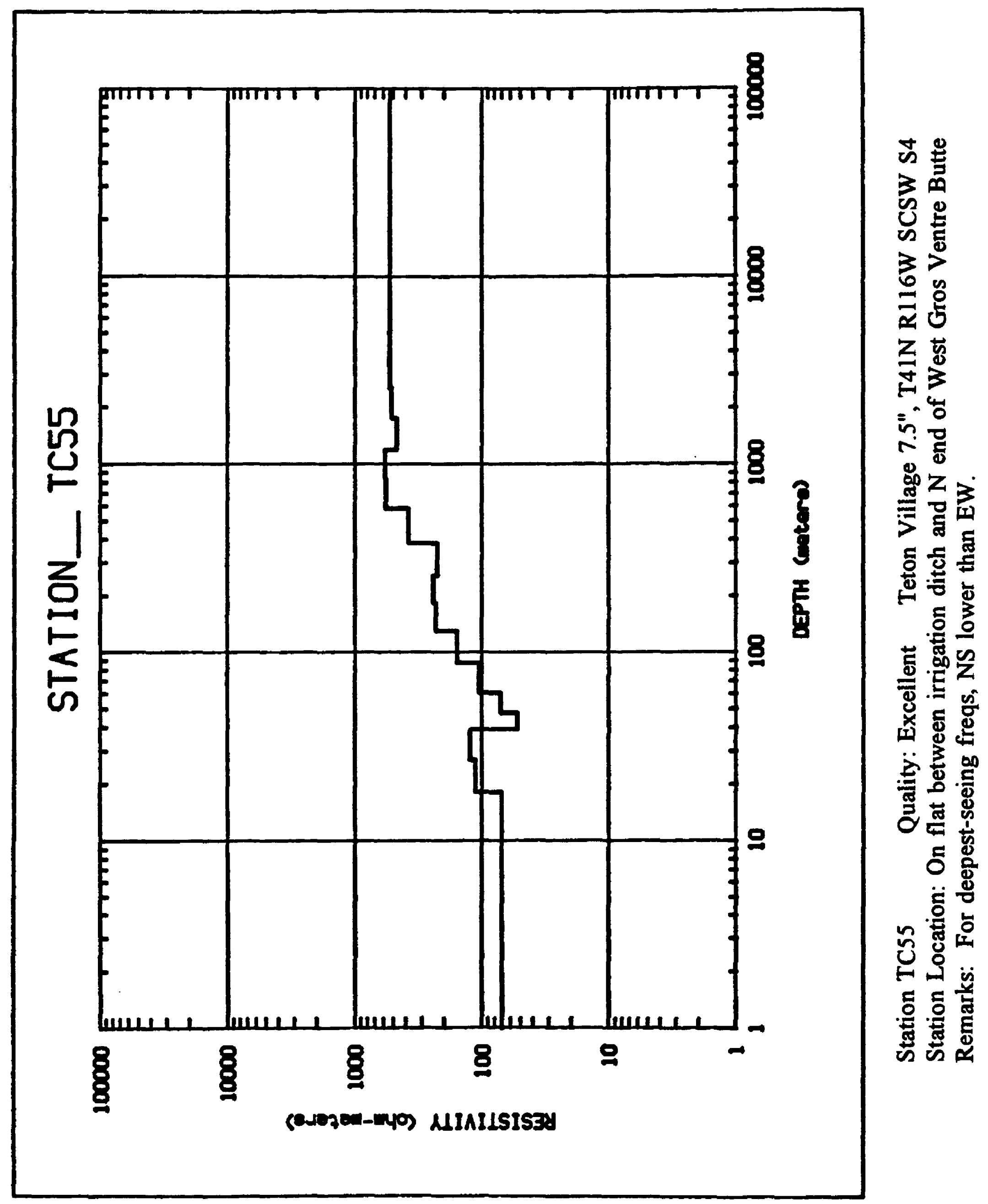




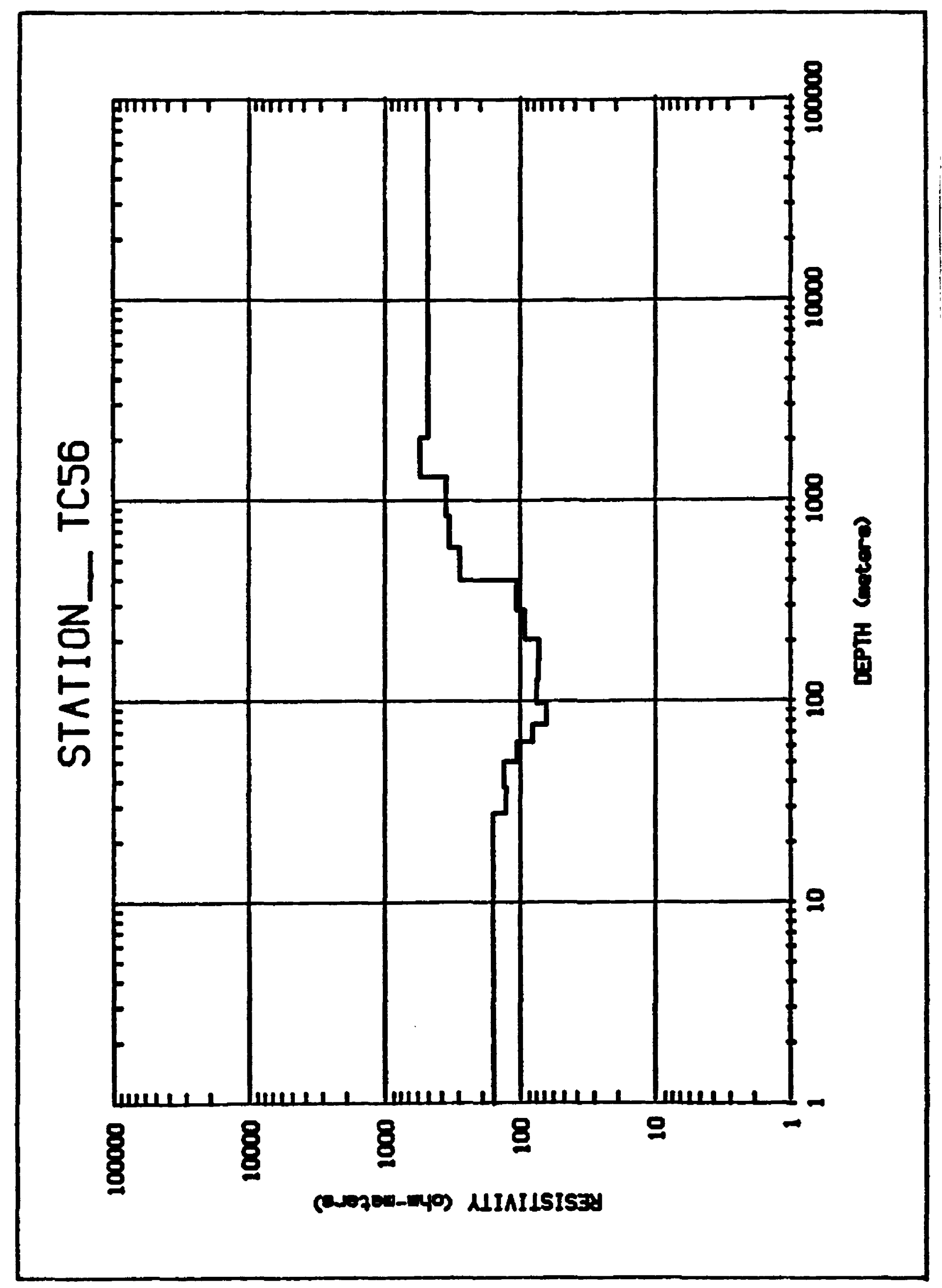

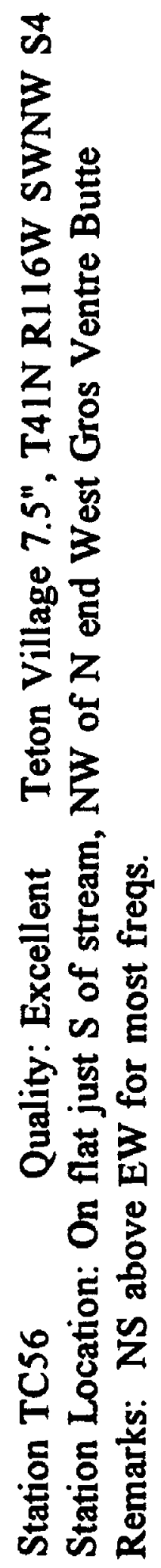




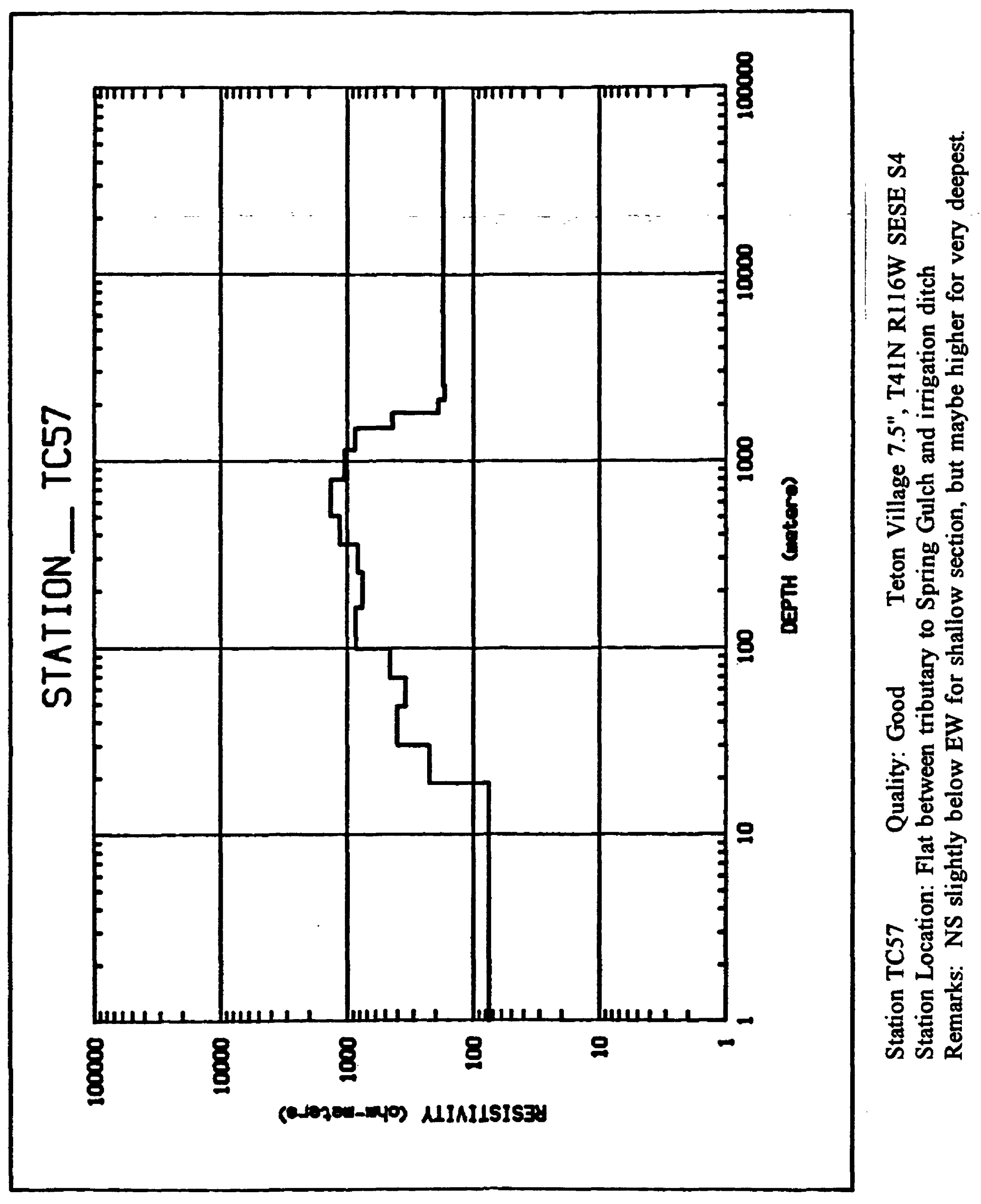




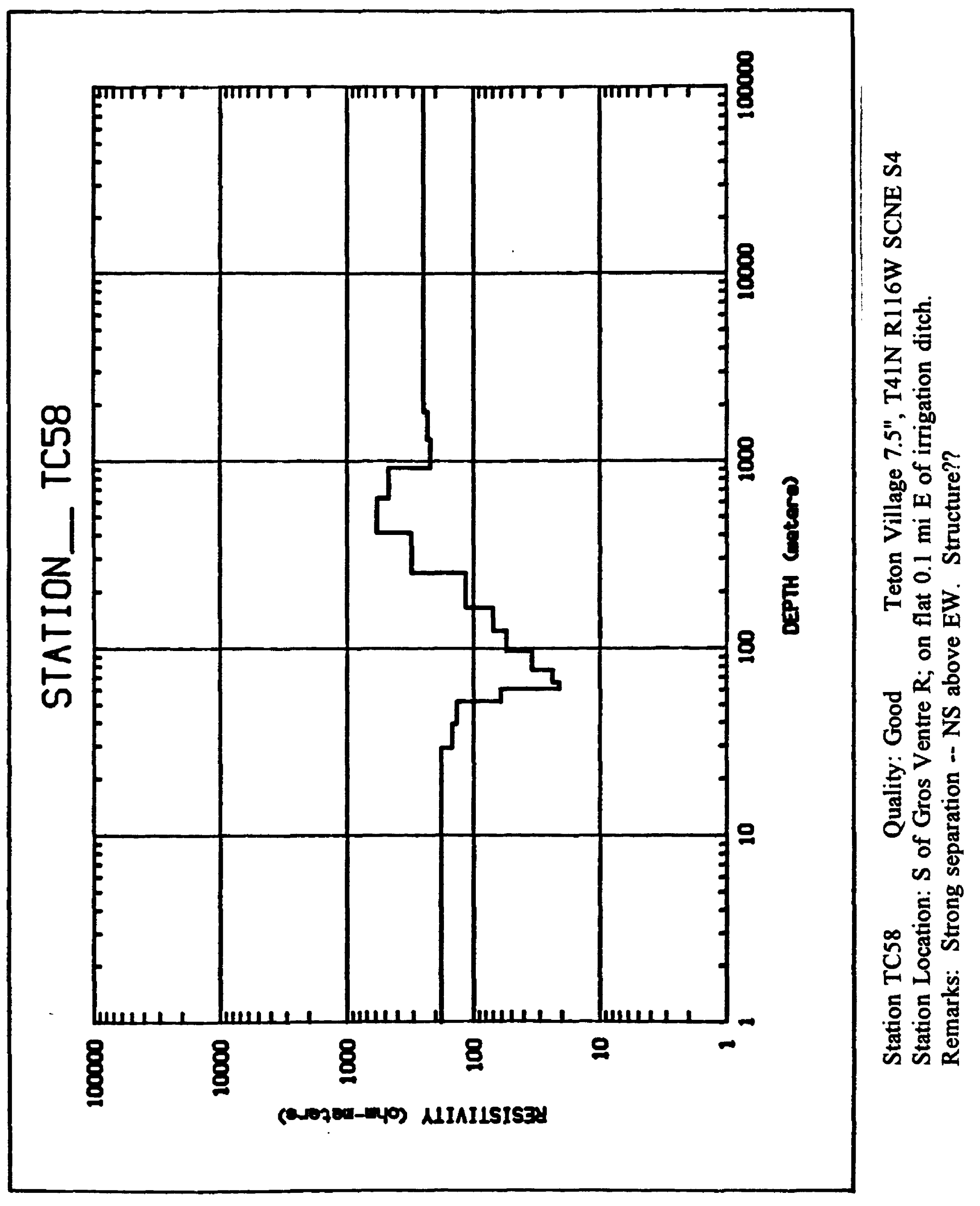




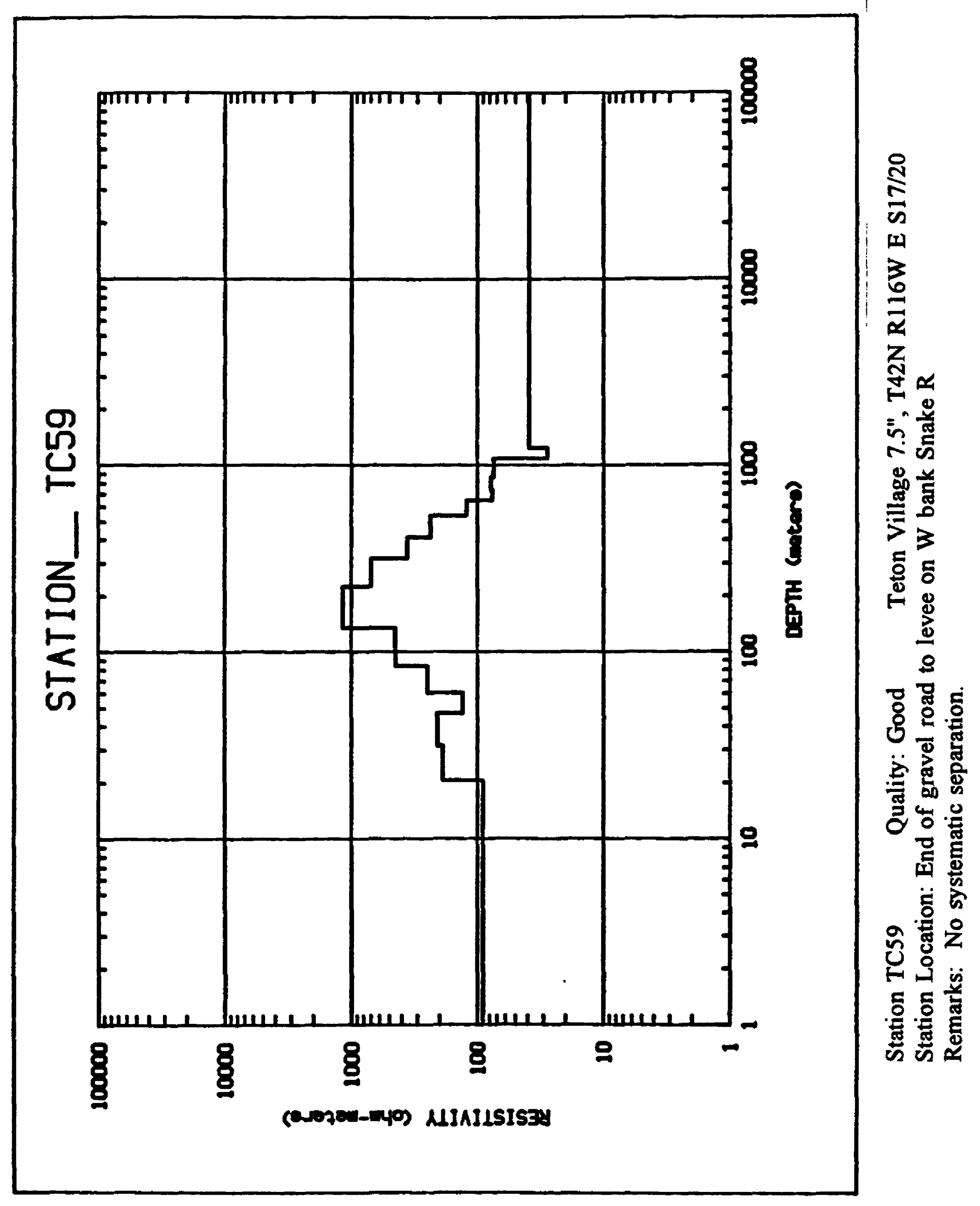




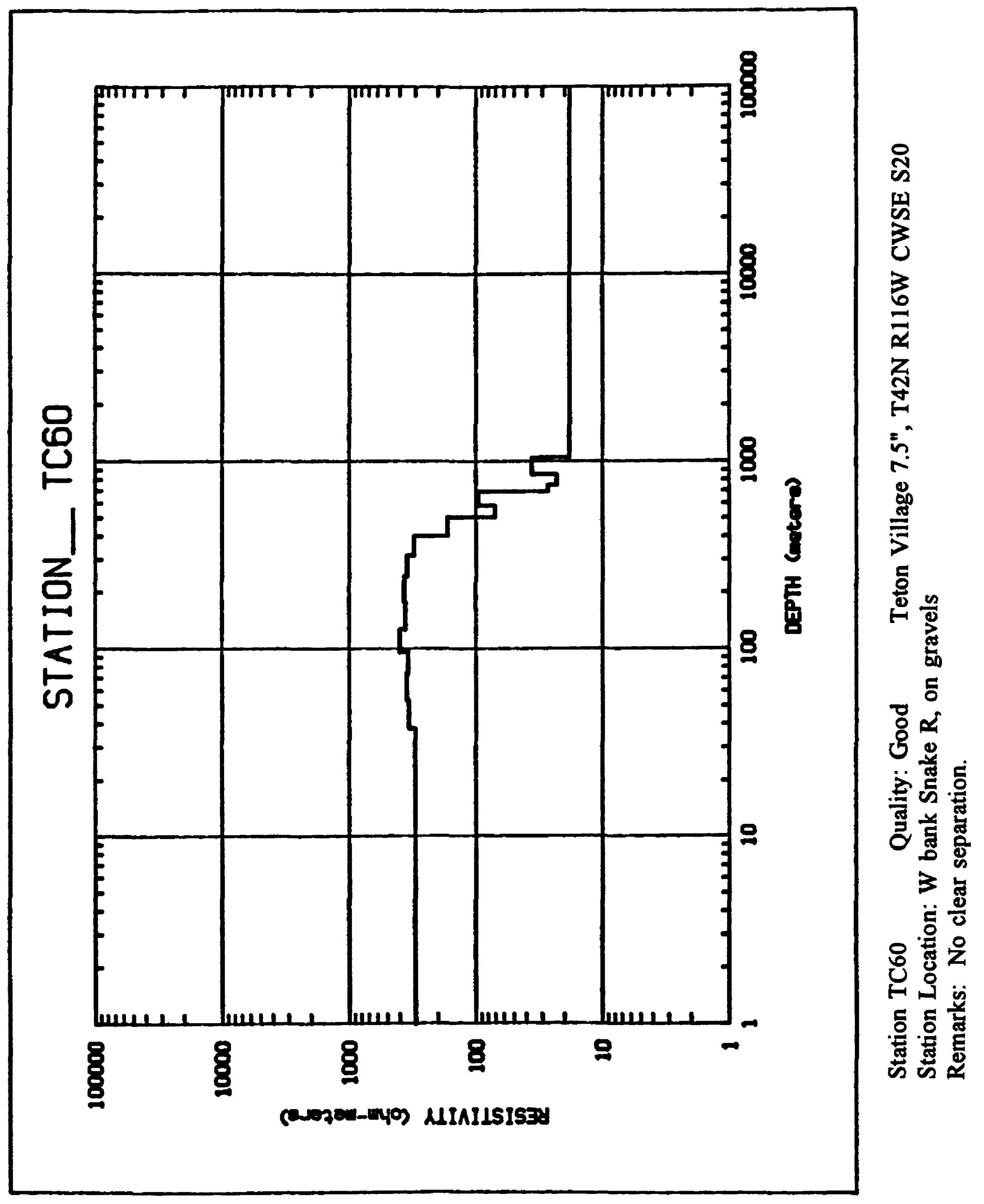




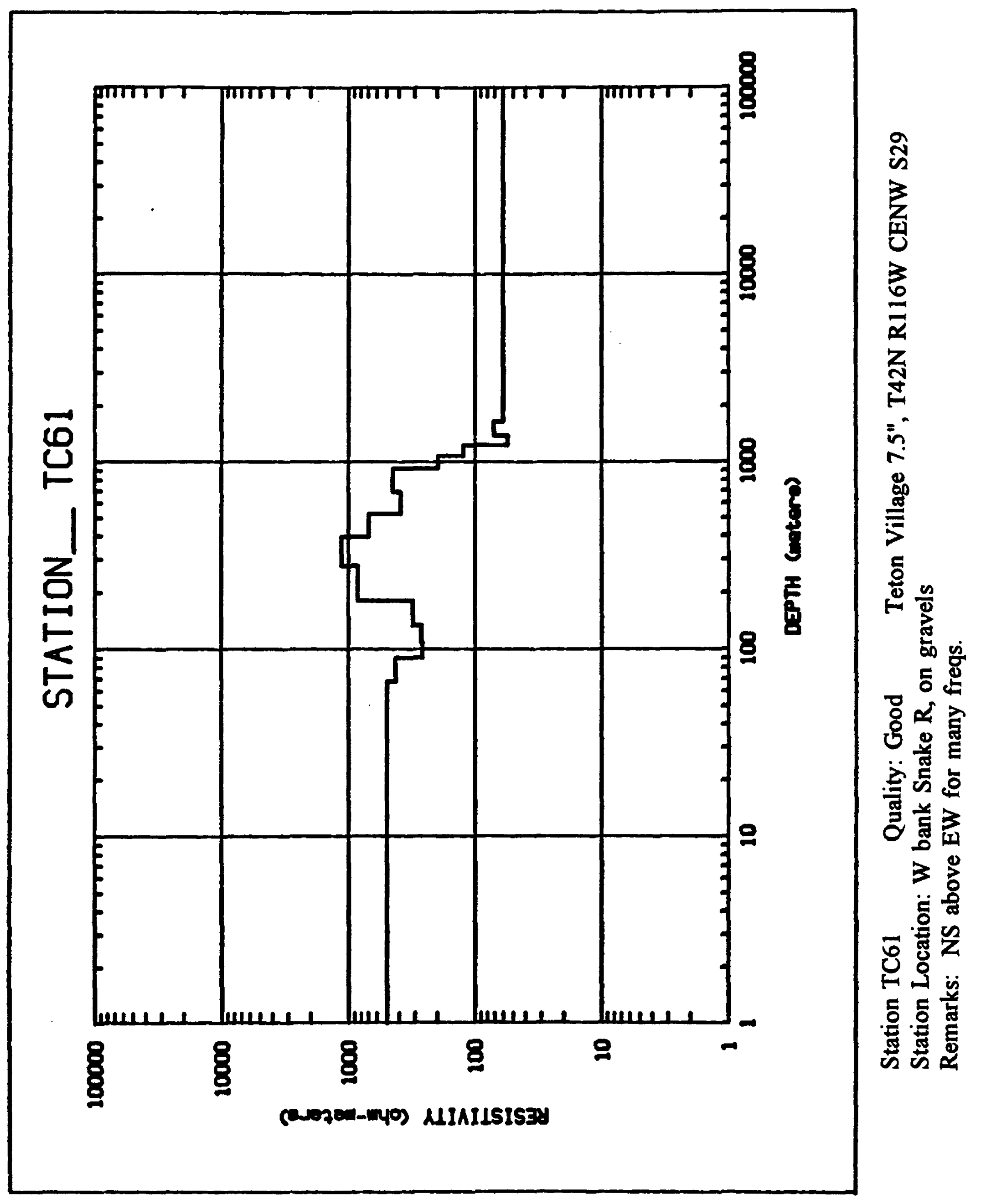




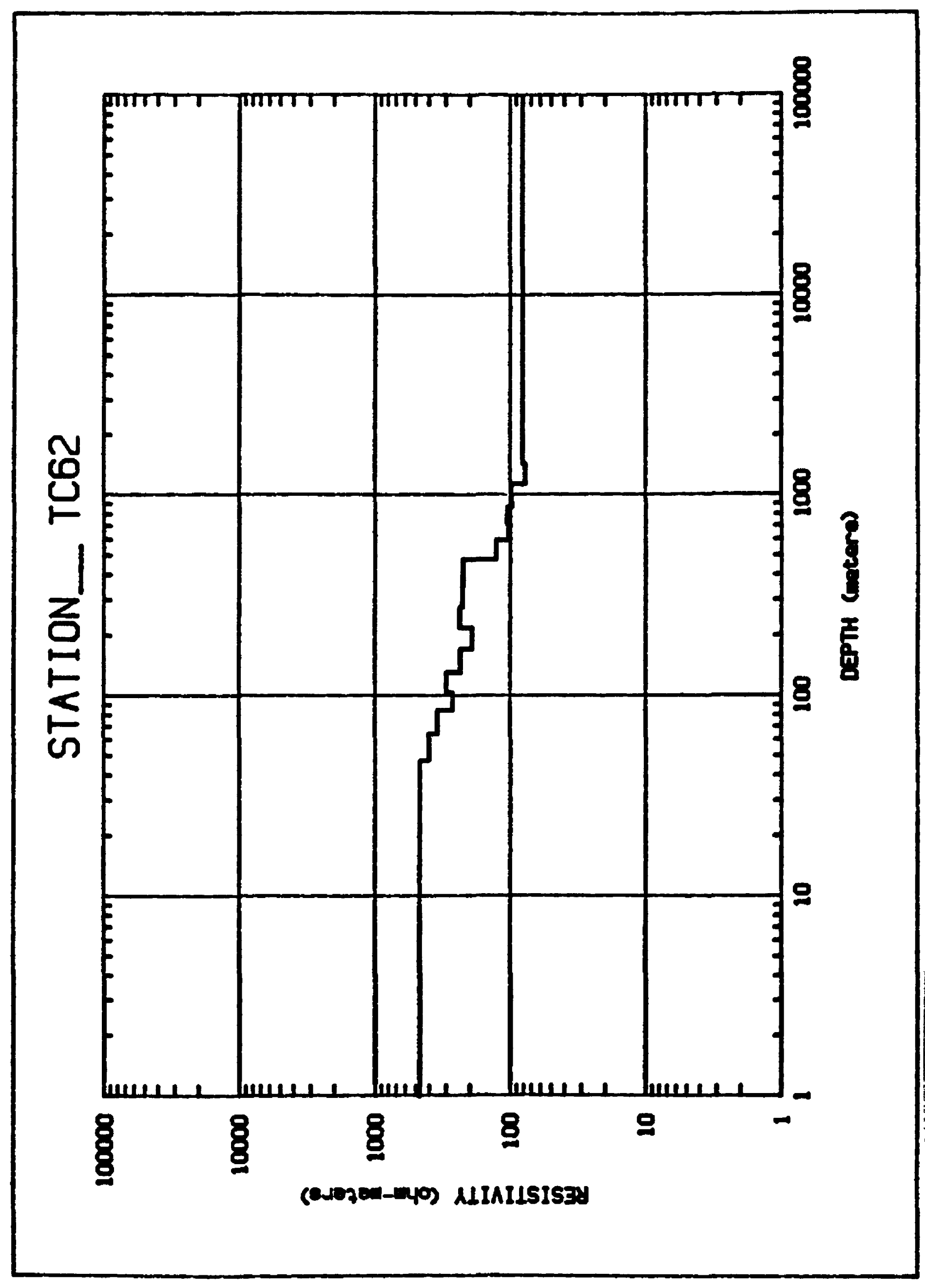

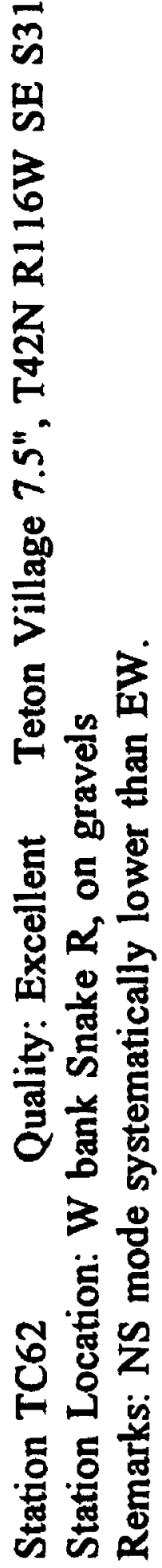




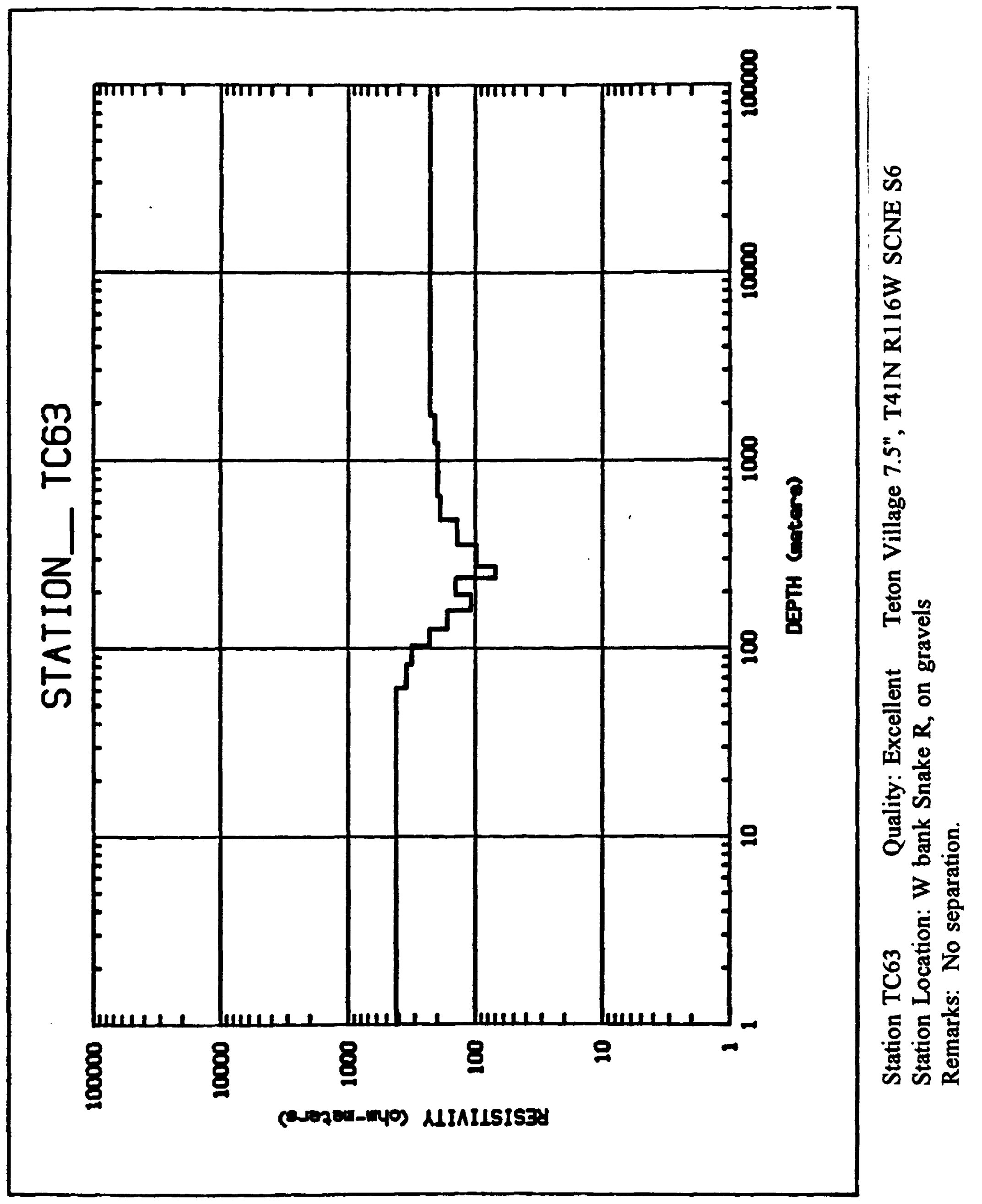




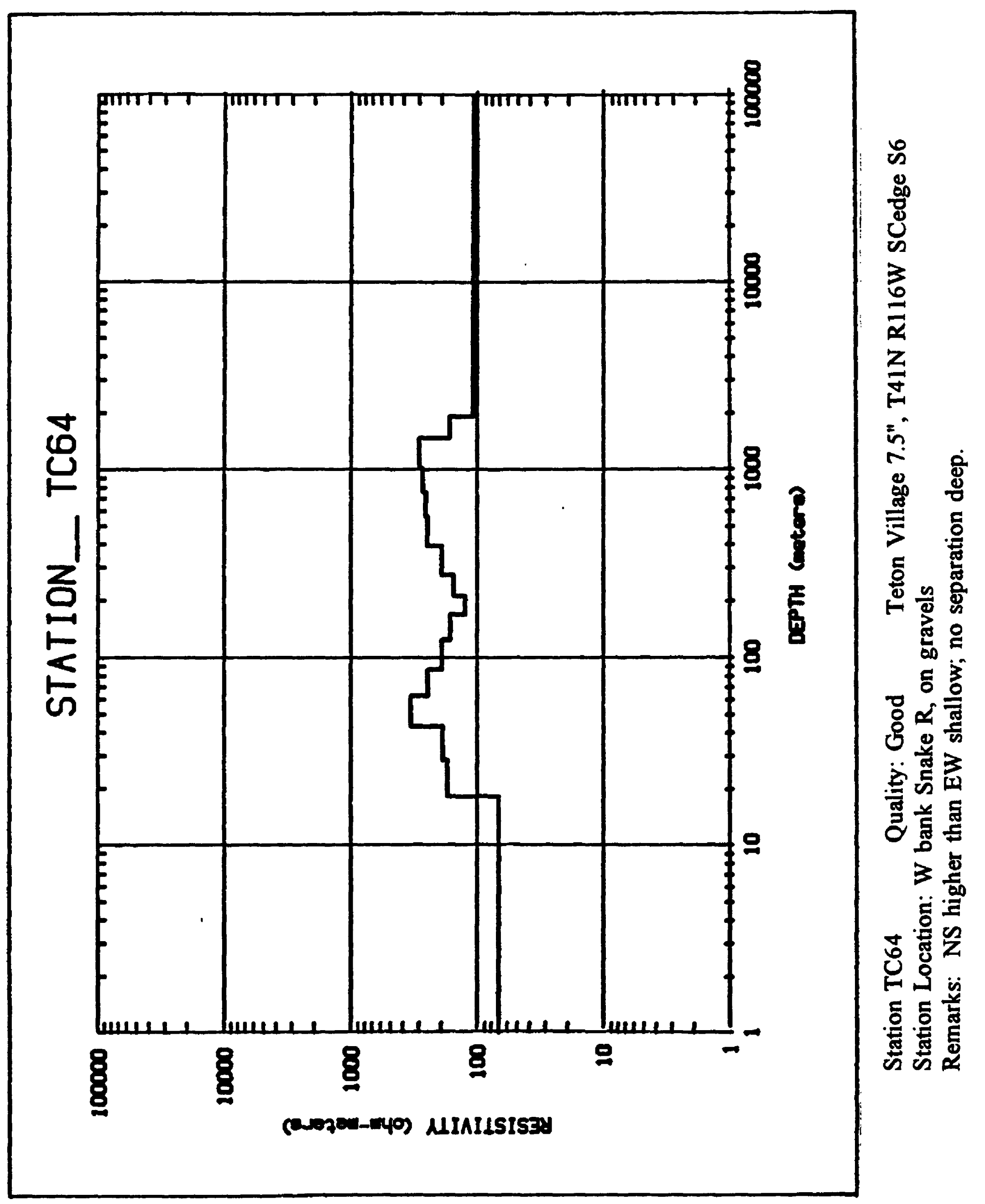




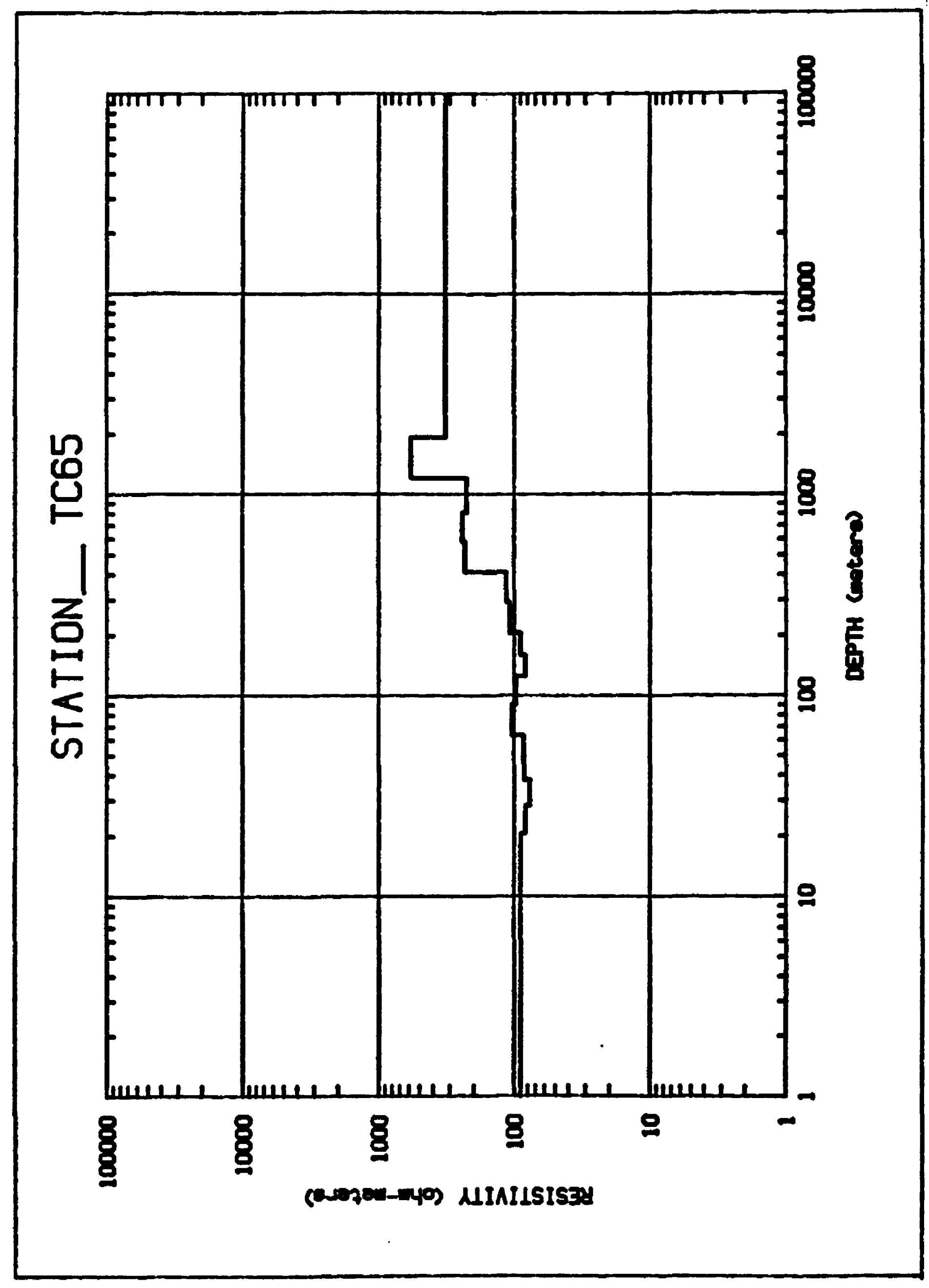

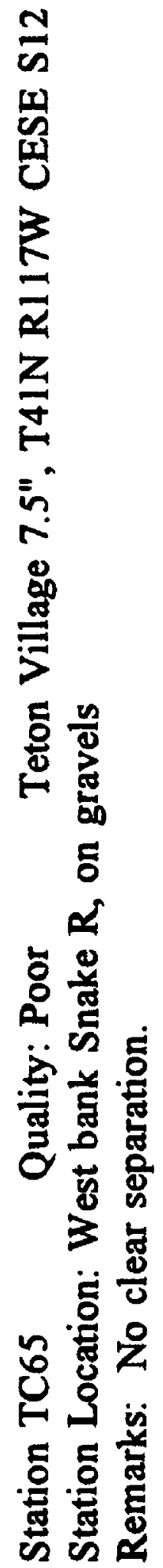




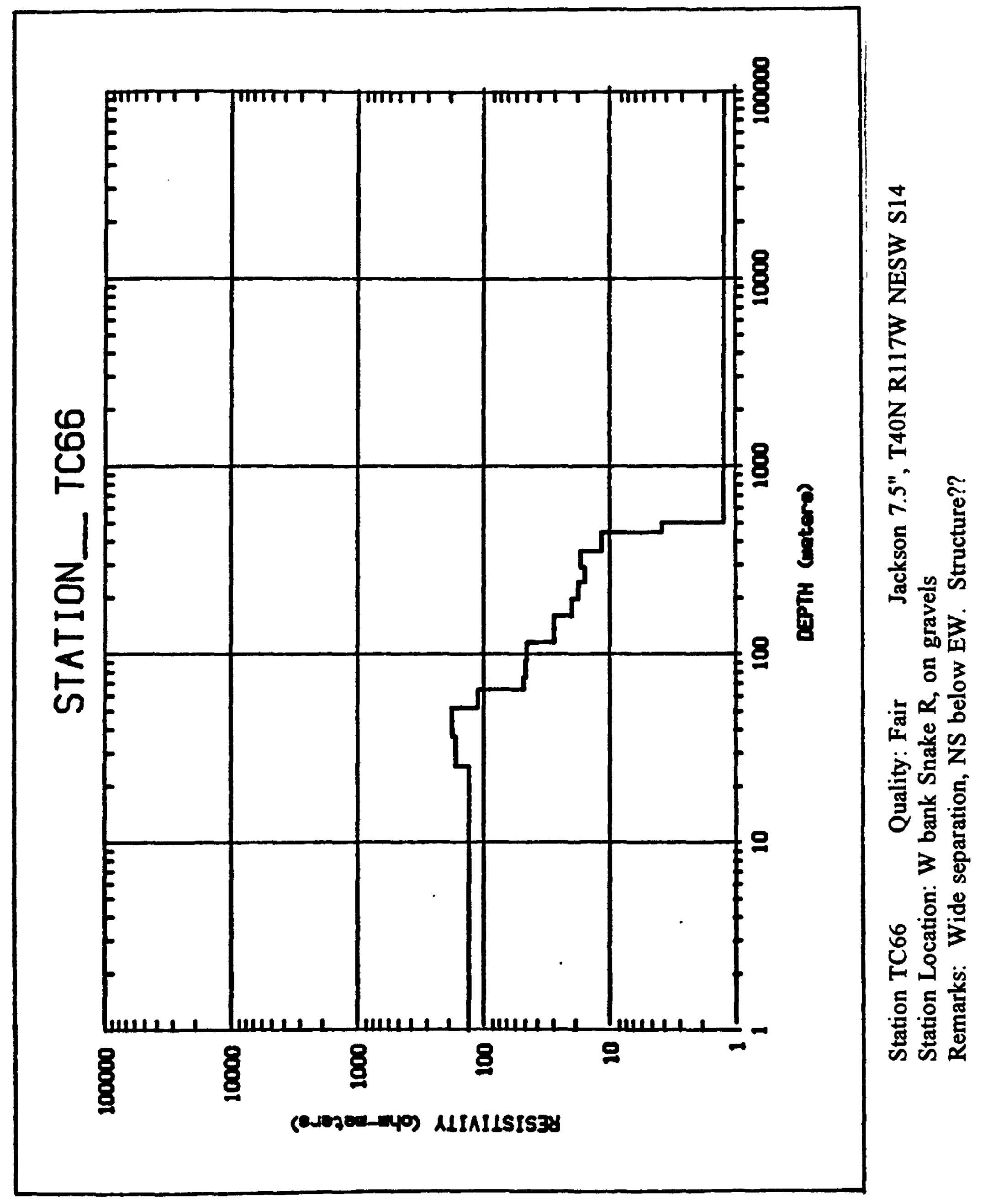




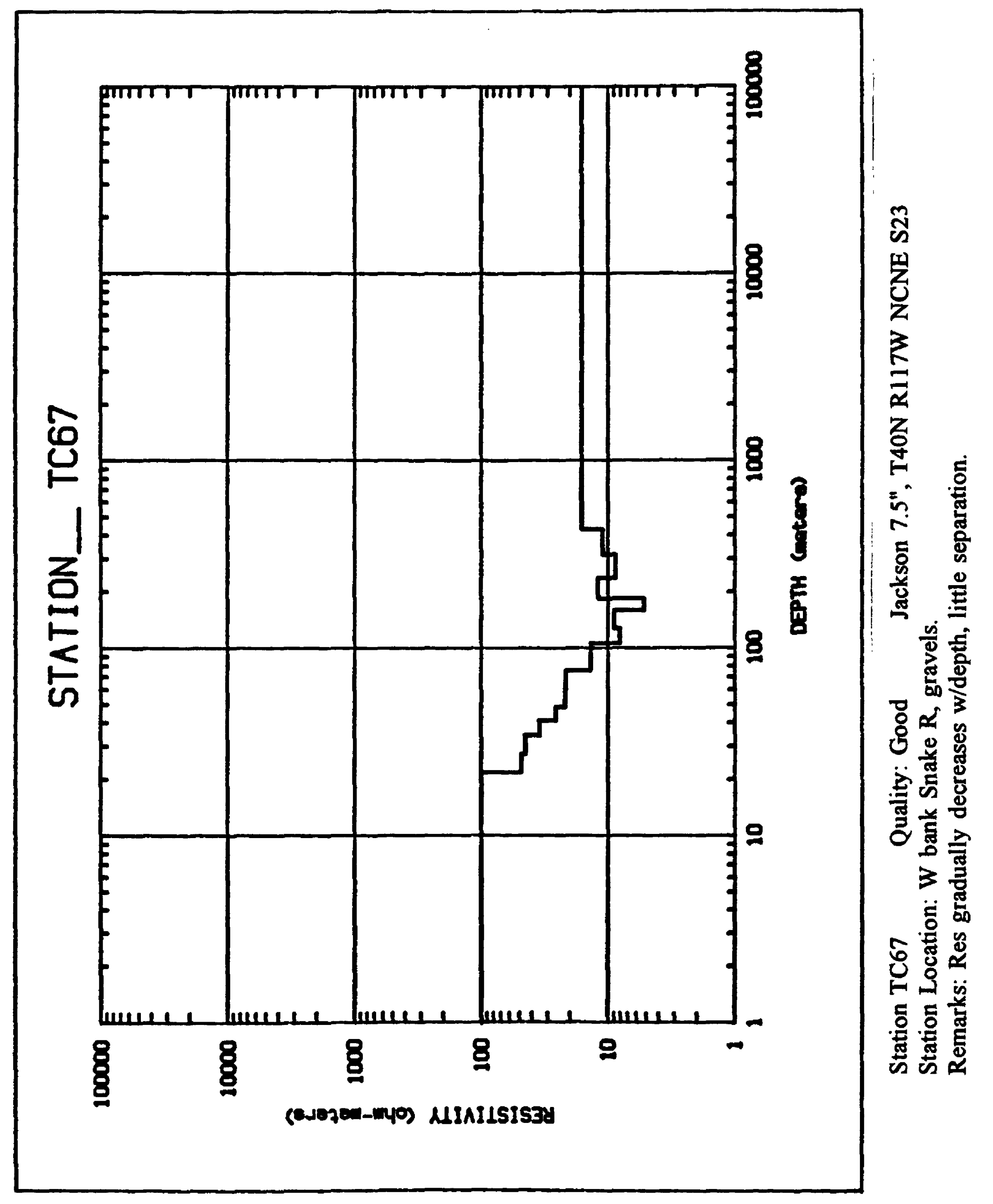




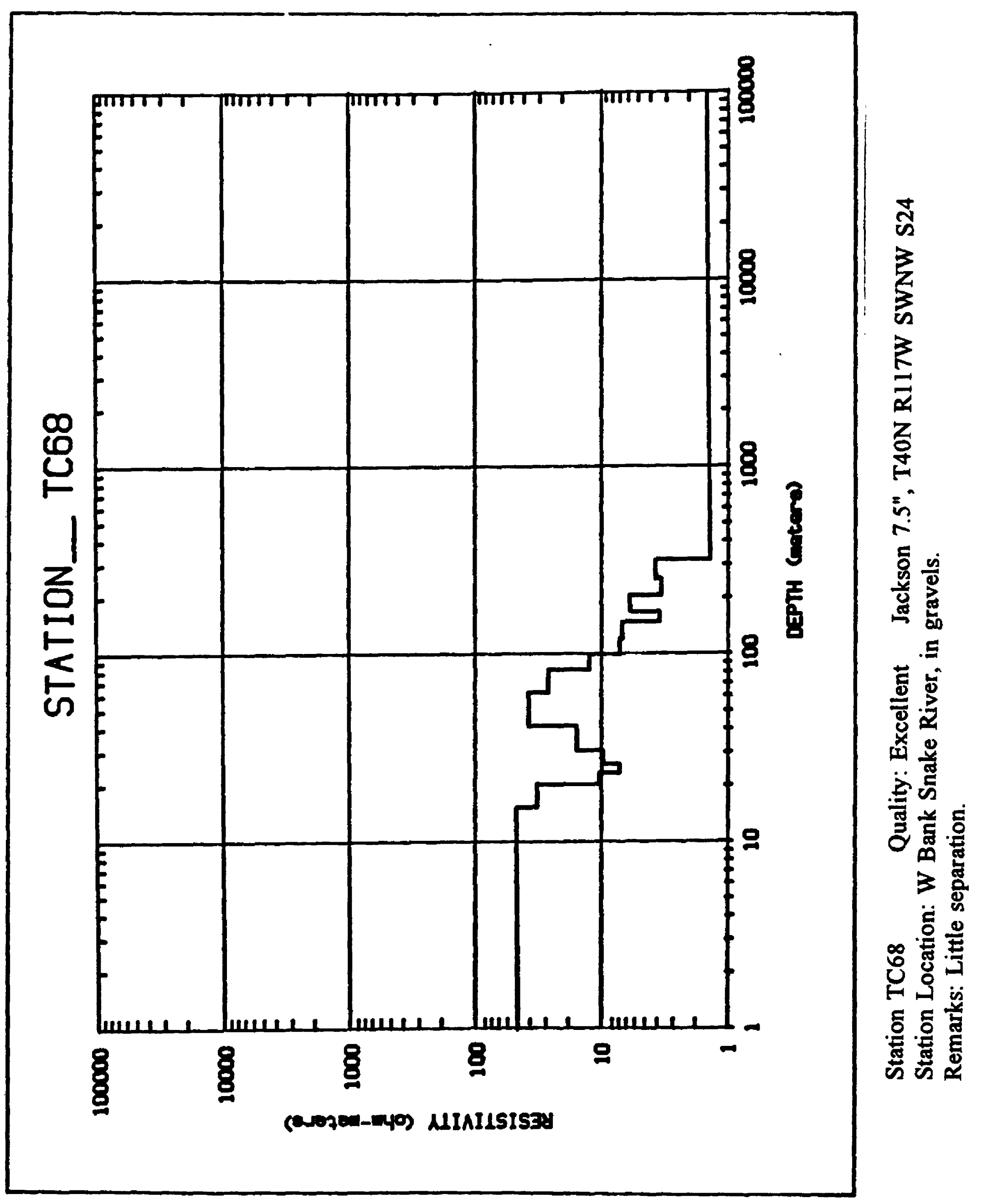




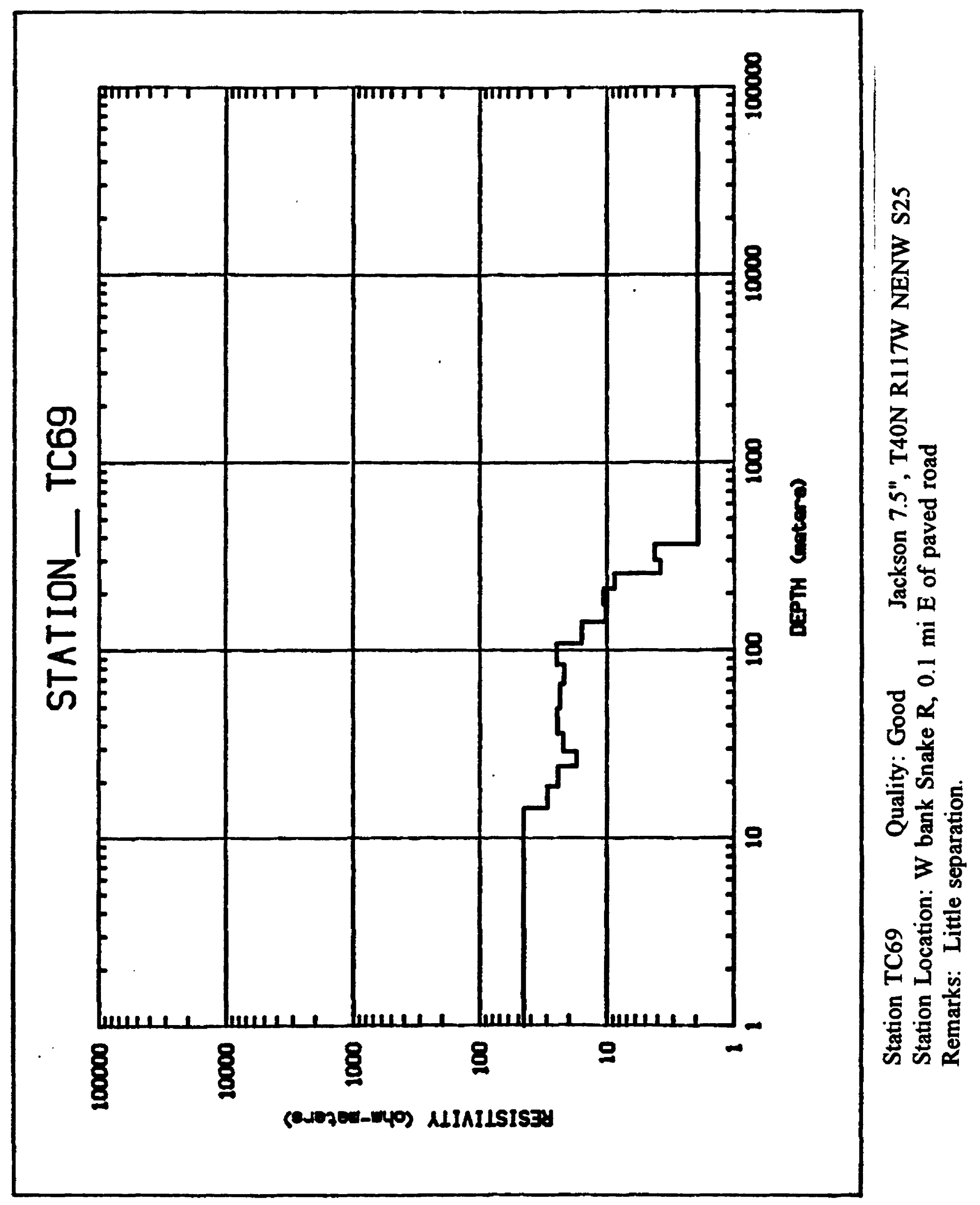




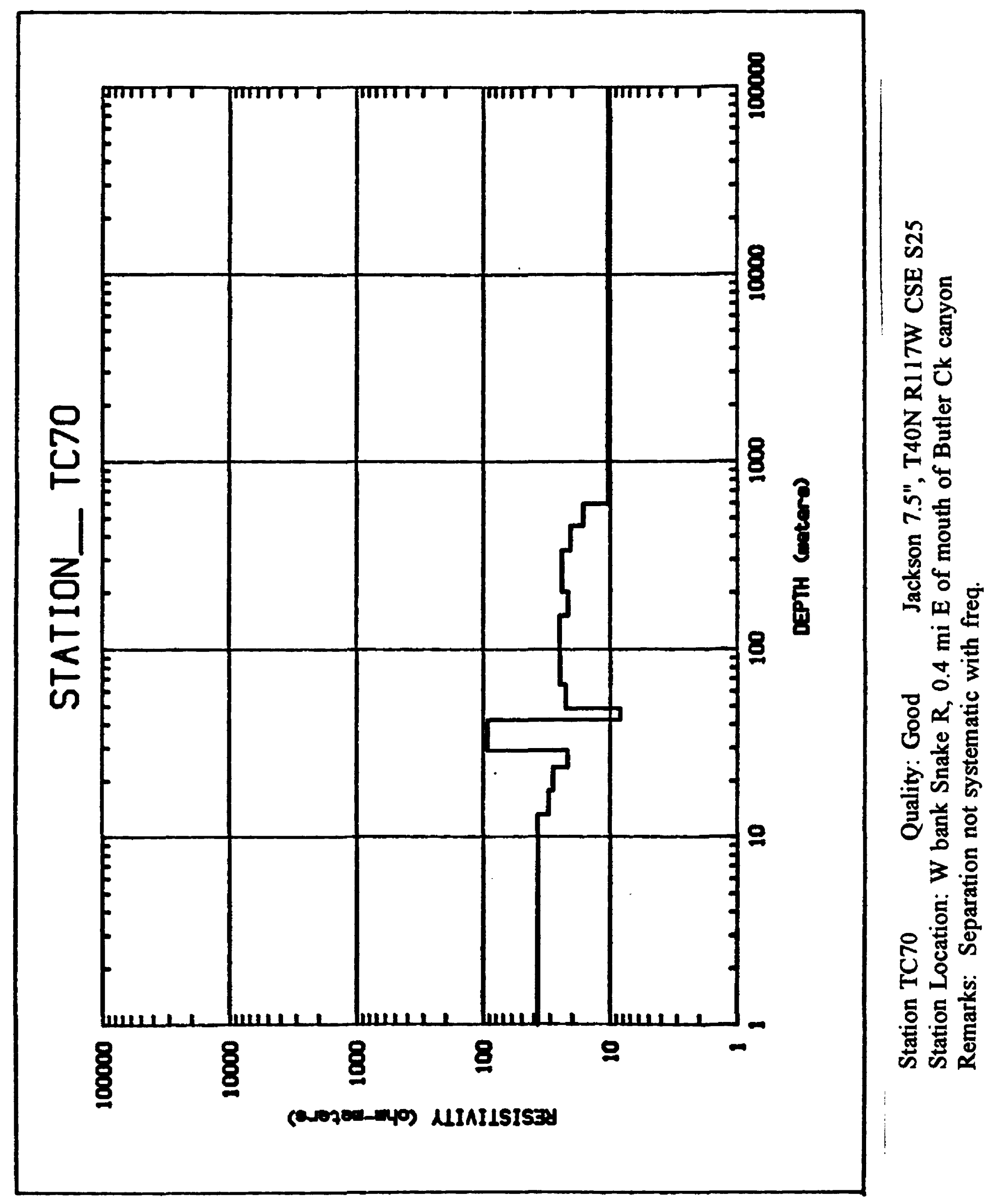




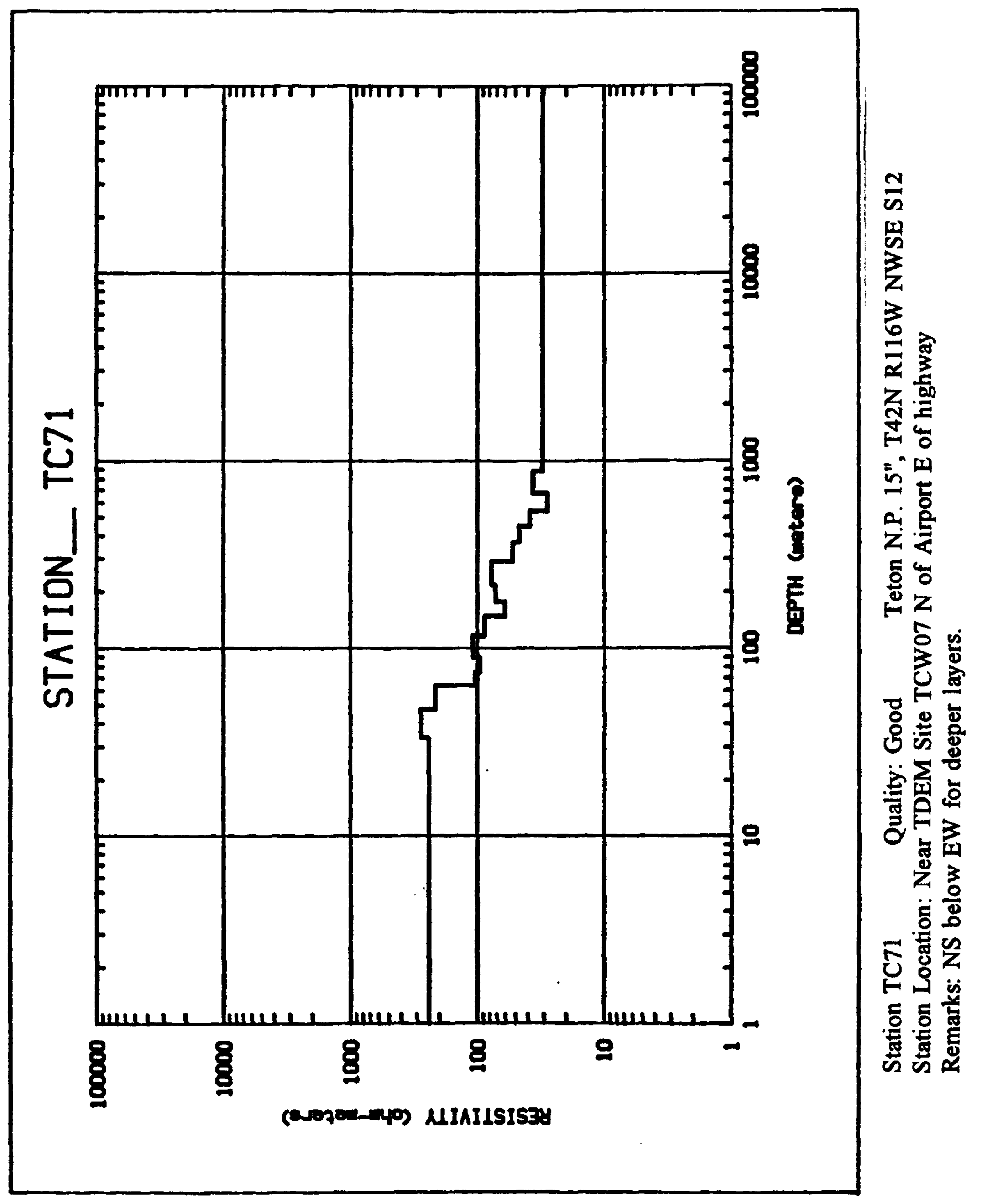




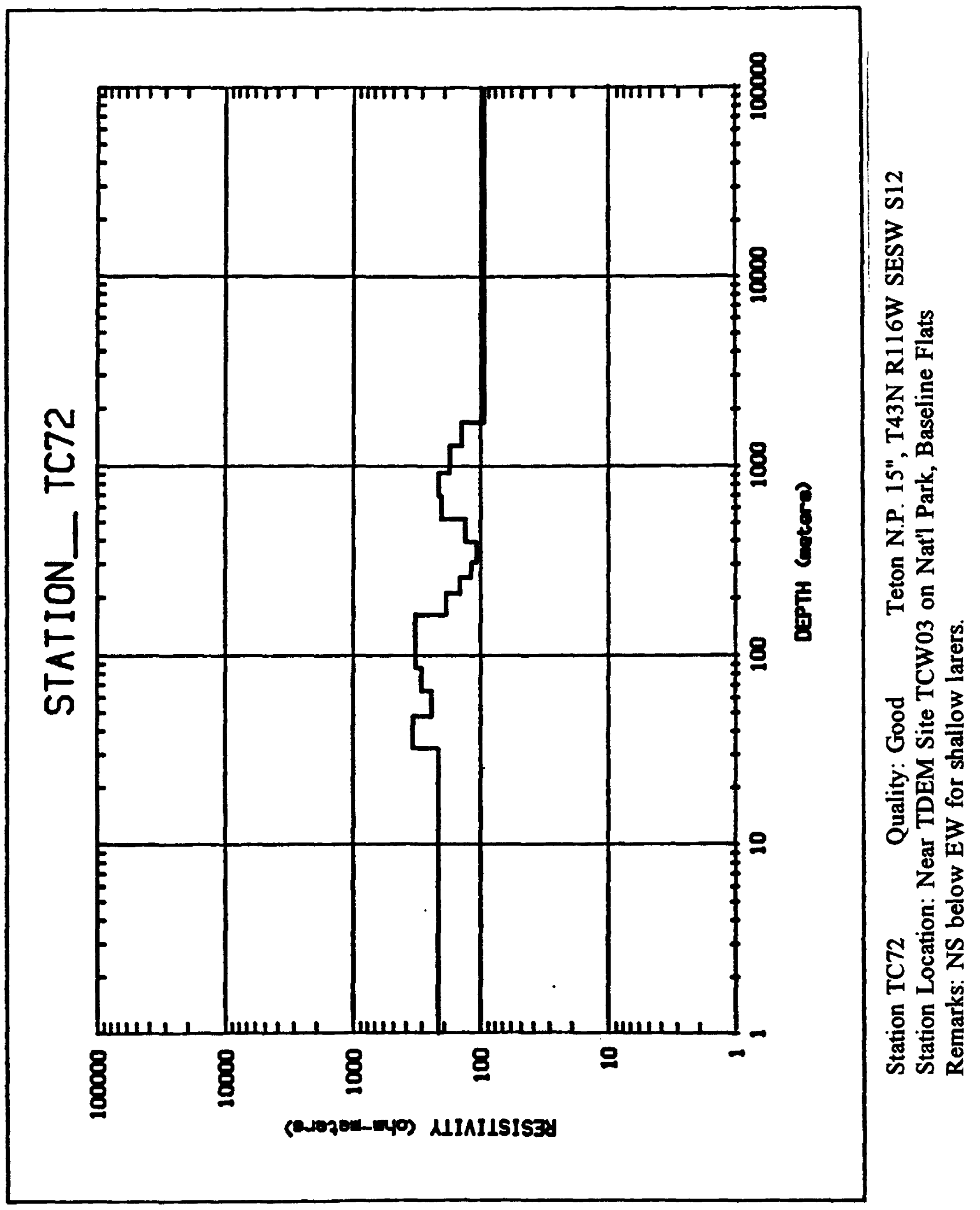




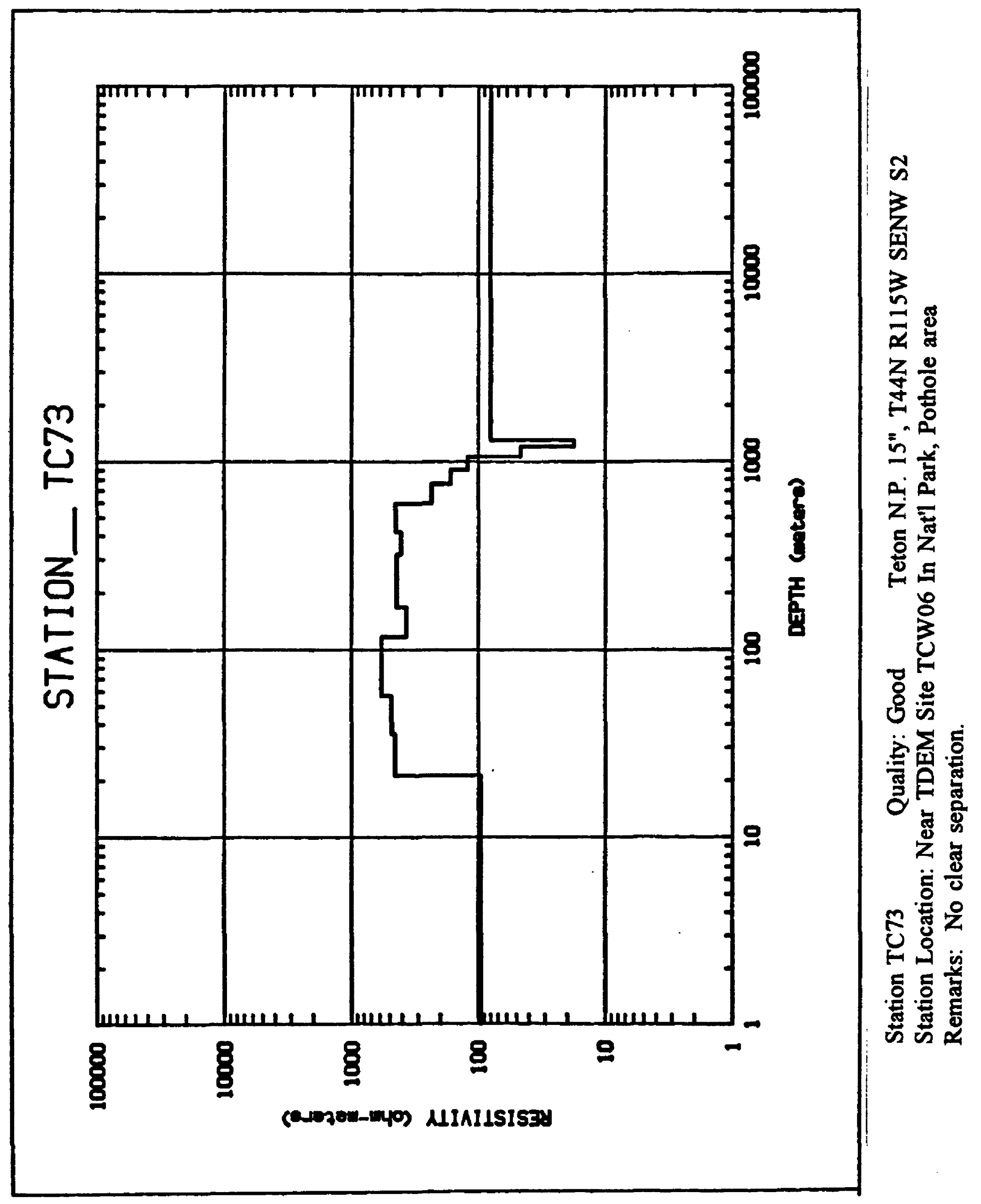




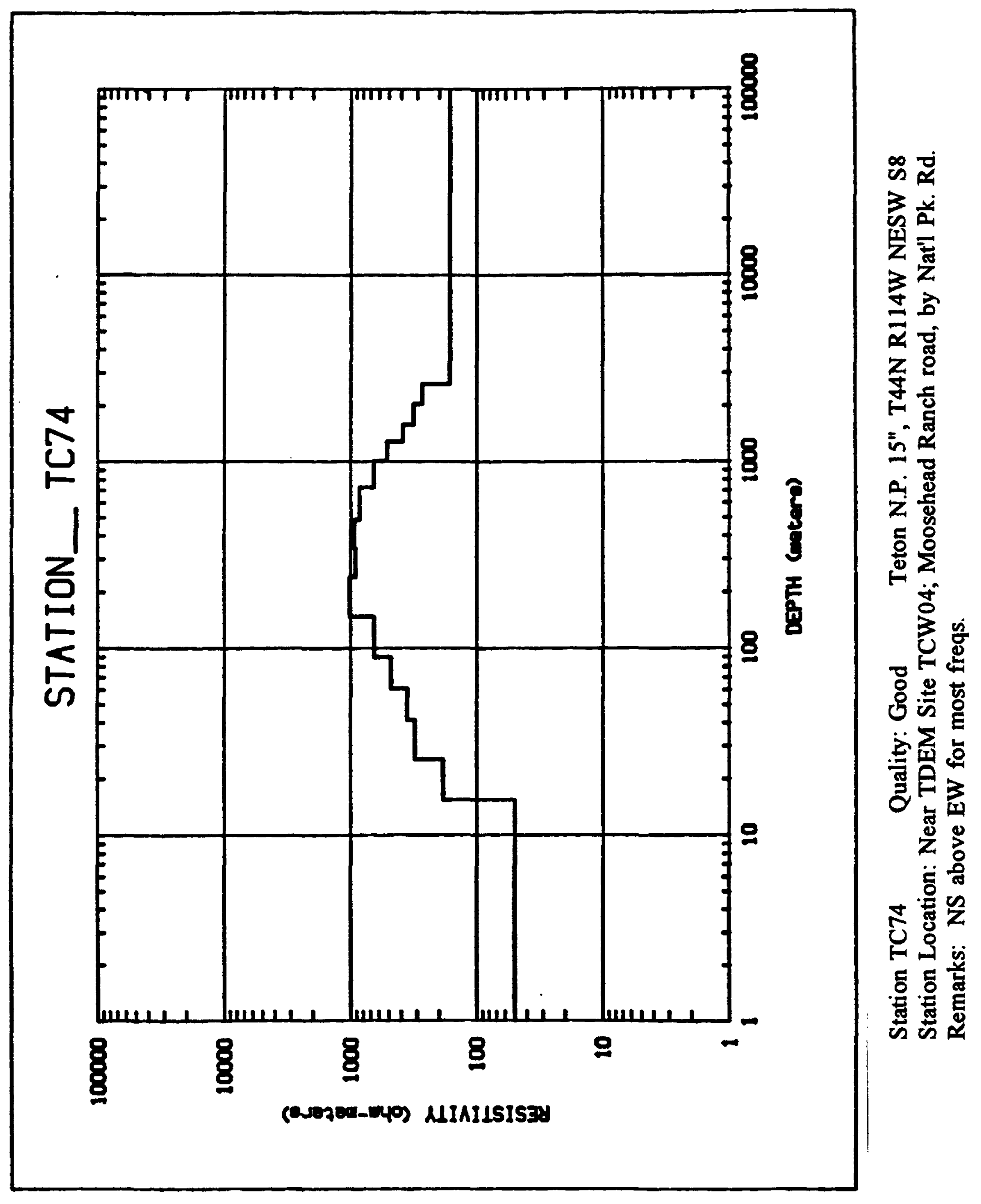




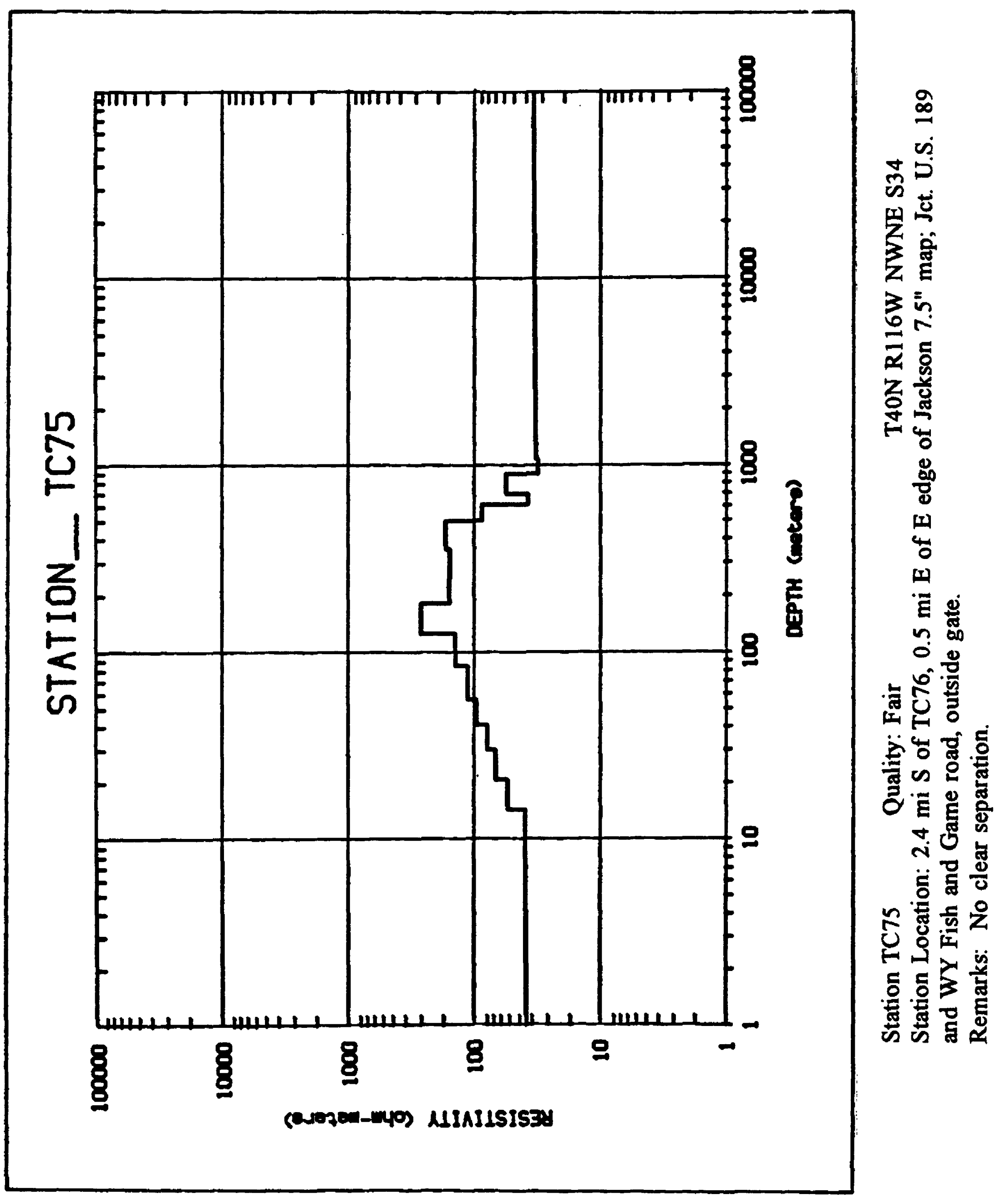




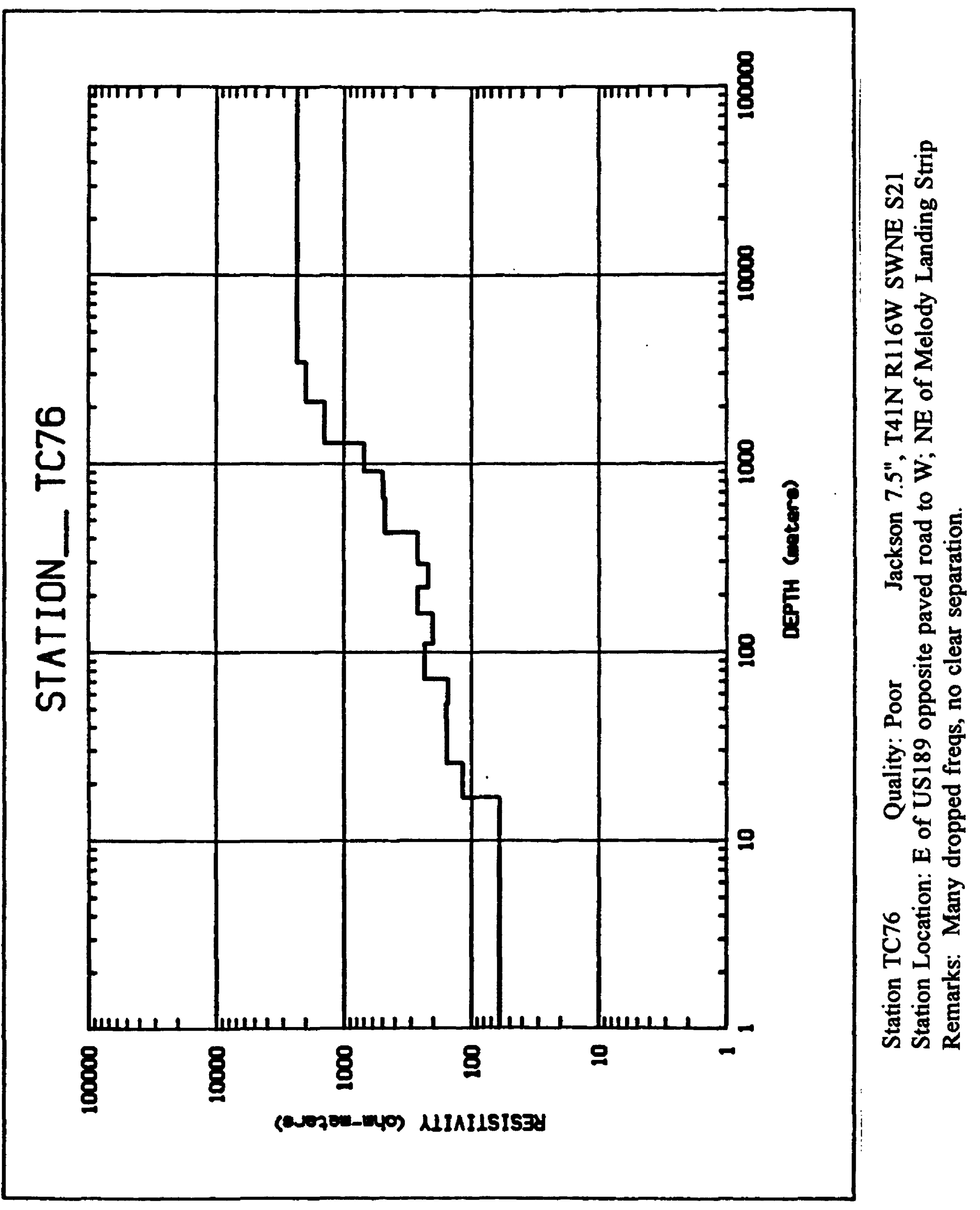




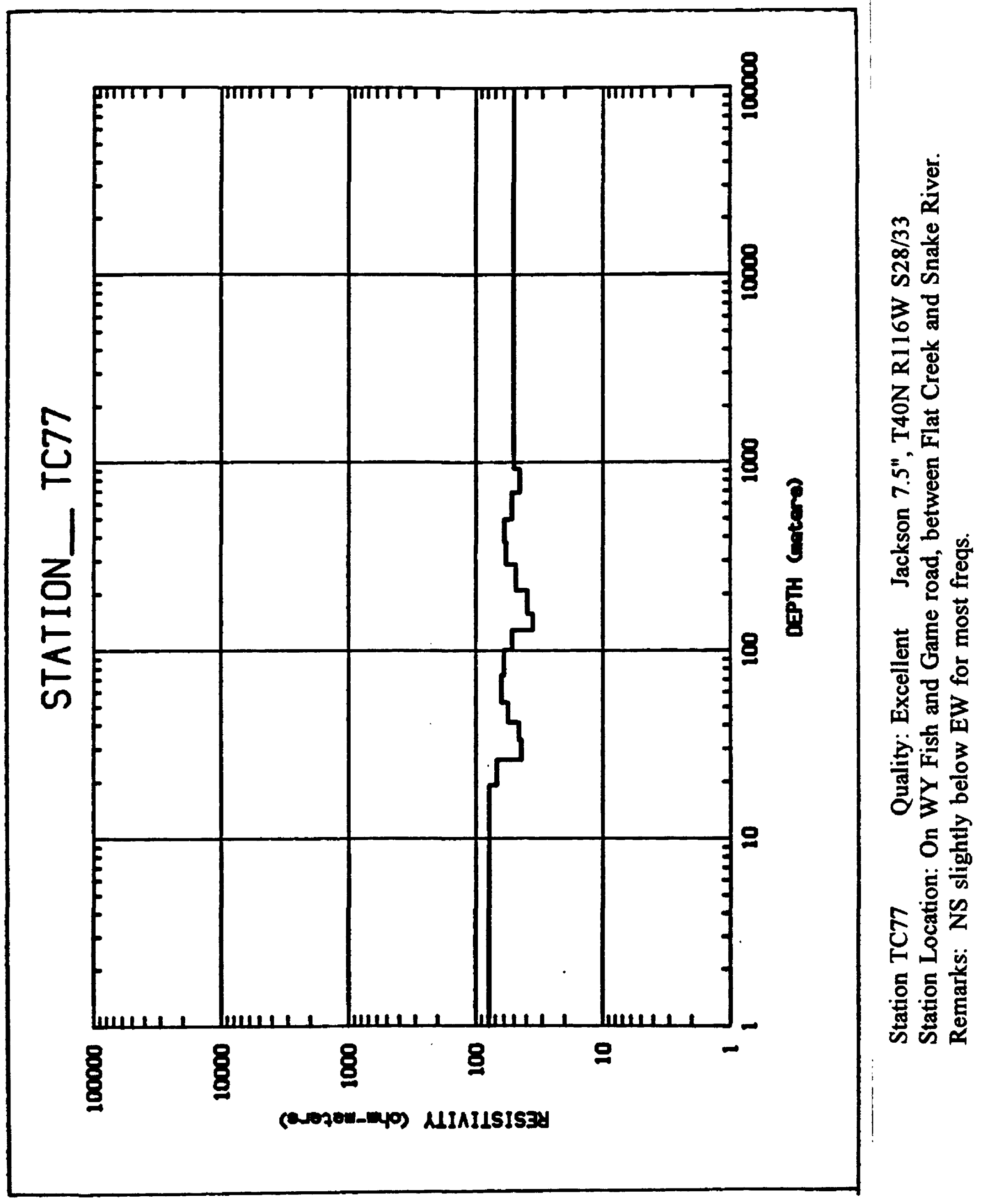




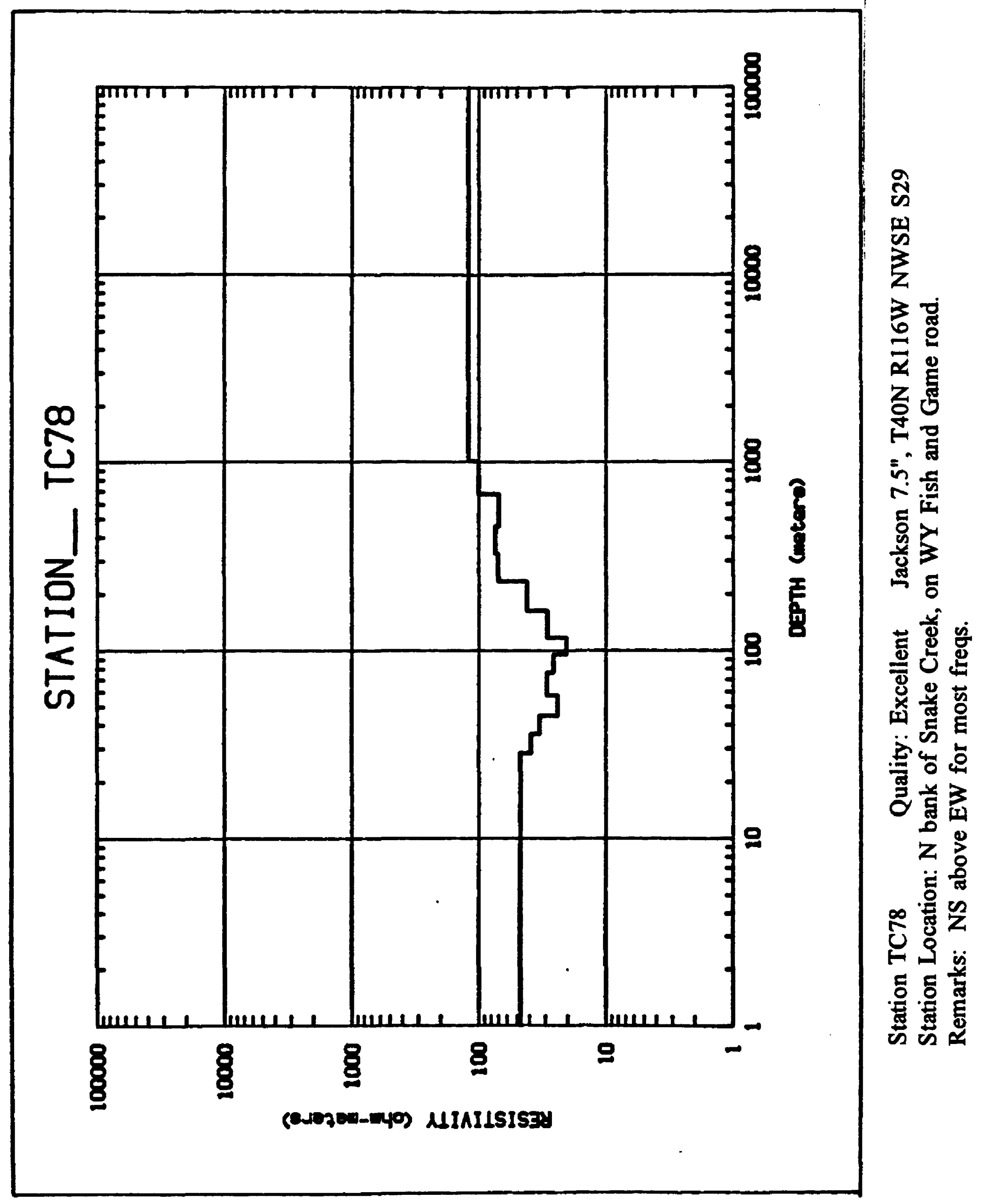




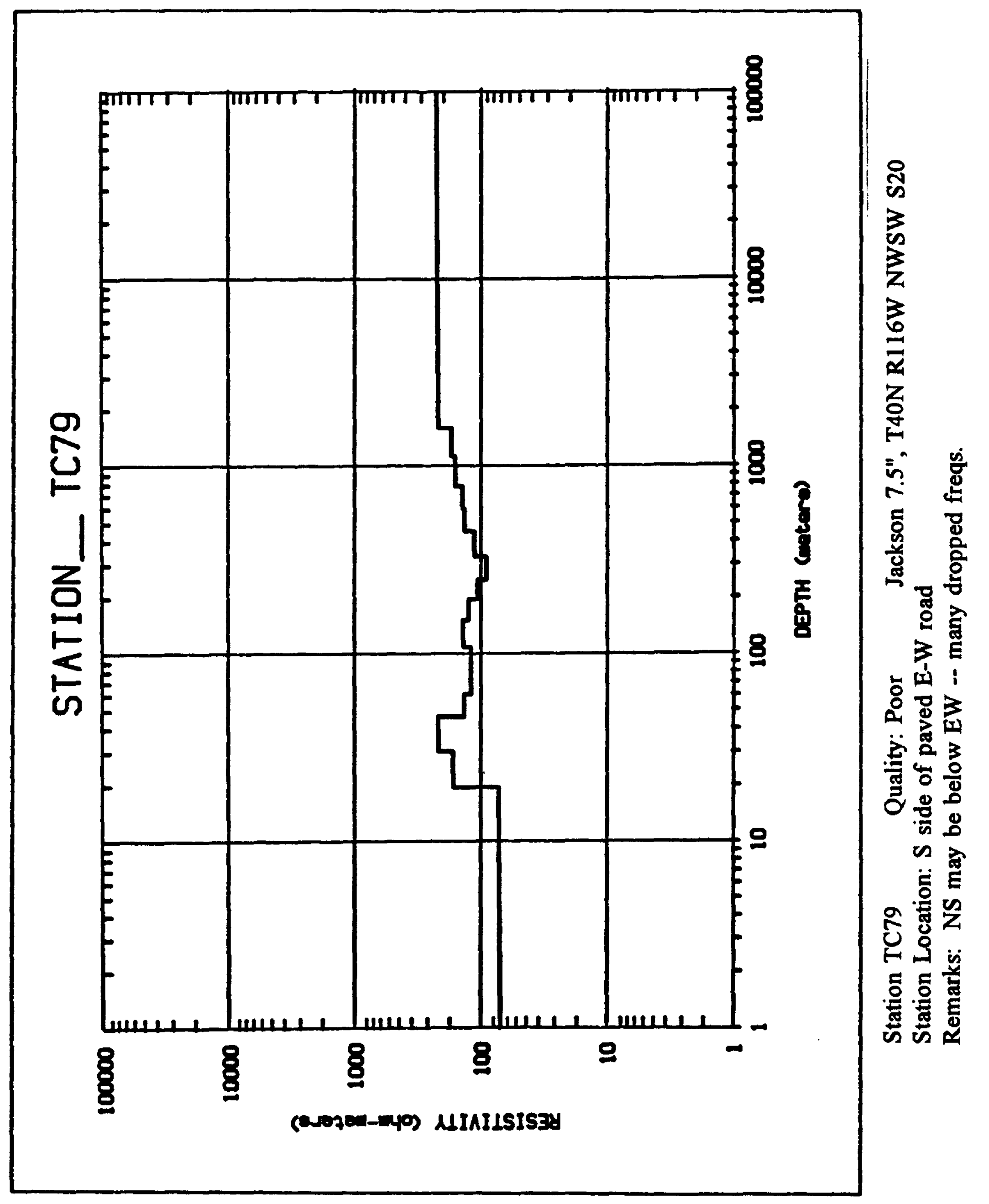




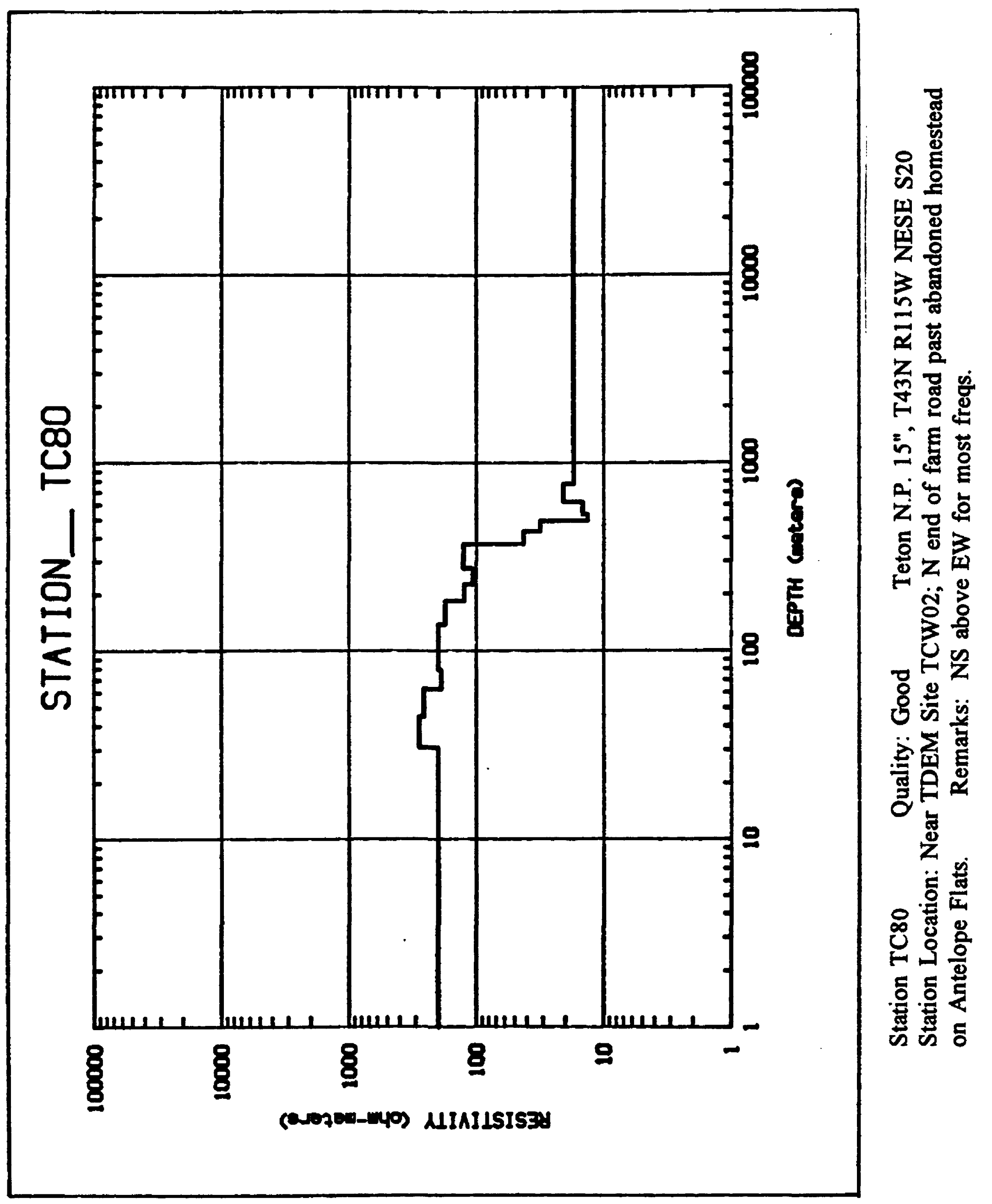




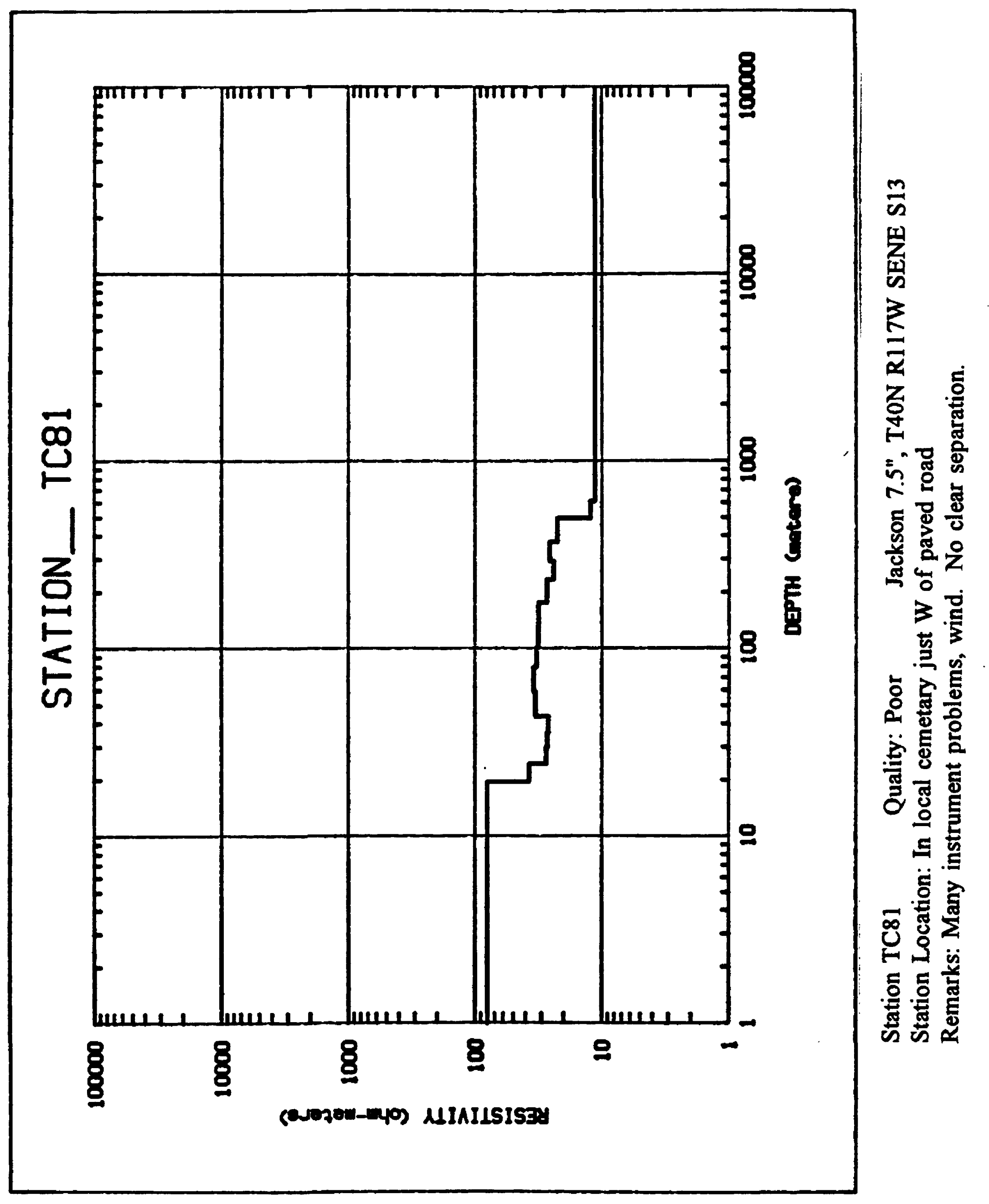




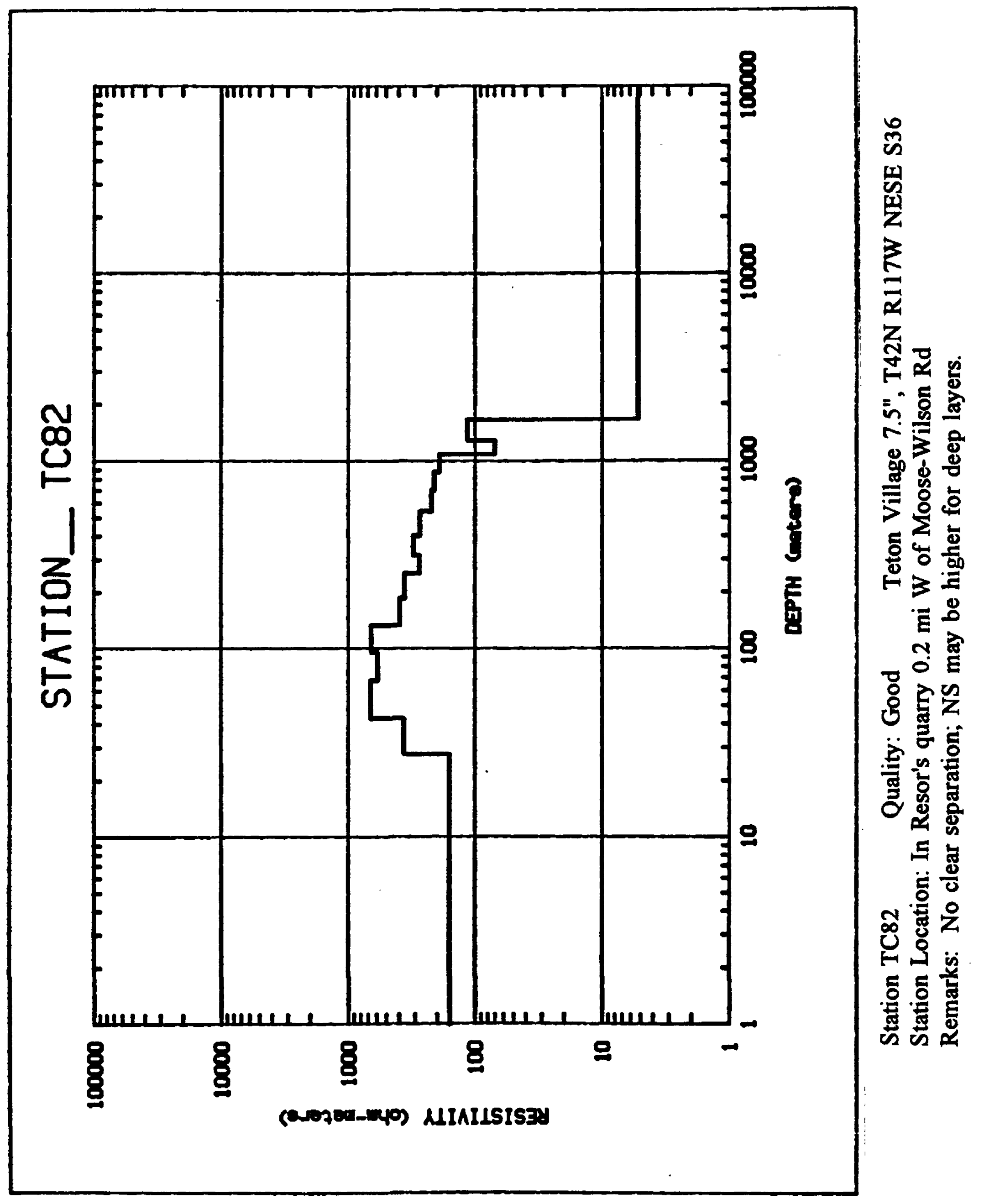

